Portland State University

PDXScholar

Summer 8-1-2013

\title{
An Evaluation of the Nontarget Effects of Transgenic Bacillus thuringiensis Maize on Arbuscular Mycorrhizal Fungi in the Soil Ecosystem
}

Tanya Elizabeth Amy Cheeke Portland State University

Follow this and additional works at: https://pdxscholar.library.pdx.edu/open_access_etds

Part of the Food Biotechnology Commons, Molecular, Genetic, and Biochemical Nutrition Commons, and the Other Genetics and Genomics Commons Let us know how access to this document benefits you.

\section{Recommended Citation}

Cheeke, Tanya Elizabeth Amy, "An Evaluation of the Nontarget Effects of Transgenic Bacillus thuringiensis Maize on Arbuscular Mycorrhizal Fungi in the Soil Ecosystem" (2013). Dissertations and Theses. Paper 1027.

https://doi.org/10.15760/etd.1027

This Dissertation is brought to you for free and open access. It has been accepted for inclusion in Dissertations and Theses by an authorized administrator of PDXScholar. Please contact us if we can make this document more accessible: pdxscholar@pdx.edu. 
An Evaluation of the Nontarget Effects of Transgenic Bacillus thuringiensis Maize on Arbuscular Mycorrhizal Fungi in the Soil Ecosystem

by

Tanya Elizabeth Amy Cheeke

A dissertation submitted in partial fulfillment of the requirements for the degree of

Doctor of Philosophy

in

Biology

Dissertation Committee:

Mitchell B. Cruzan, Chair

Todd Rosenstiel

Lisa Weasel

Daniel Ballhorn

James Bever

William Fish

Portland State University

2013 
(C) 2013 Tanya Elizabeth Amy Cheeke 


\begin{abstract}
My dissertation research examined the effect of the cultivation of insect-resistant Bacillus thuringiensis $(\mathrm{Bt})$ maize on the soil environment with a goal of understanding how to obtain a balance between technological advancement and maintenance of a healthy soil ecosystem. Although $B t$ plants may help to reduce pesticide use, conferring benefits to farm workers and the environment, there are still unresolved questions about how the cultivation of $B t$ plants affects soil organisms. For this dissertation project, I used 14 different genotypes of $B t$ maize and non-Bt maize (Zea mays) to investigate the effects of transgenic $B t$ plants on the colonization ability, abundance, and diversity of symbiotic arbuscular mycorrhizal fungi (AMF) in the soil ecosystem over time. My greenhouse studies demonstrated that $B t$ maize plants exhibited reduced AMF colonization across multiple $B t$ genotypes and that effects were most pronounced when fertilizer levels were limited and spore density was high. In addition, I found that although differences in AMF colonization between $B t$ and non- $B t$ maize were difficult to detect in the field, spore density was reduced in $B t$ field plots after just one growing season. When I tested the effect of plot history on AMF and plant growth, I found that $B t$ and non- $B t$ maize plants had higher leaf chlorophyll content when grown in plots previously cultivated with the same maize line as the previous year, indicative of a positive feedback effect. I also examined potential mechanisms contributing to the reduced AMF colonization observed in $B t$ maize in greenhouse studies and determined that follow-up experiments should continue to investigate differences in root apoplastic invertase activity and root
\end{abstract}


permeability in $B t$ and non- $B t$ maize. Future investigations would also benefit from examining potential differences in root exudate profiles and volatile organic compounds between $B t$ and non- $B t$ cultivars. Taken together, my dissertation results suggest that, while difficult to detect in the field, reductions in AMF colonization in $B t$ maize roots may be ecologically significant as they could lead to a decrease in the abundance of AMF propagules in the soil over time, potentially impacting soil structure and function in areas where $B t$ crop cultivation is high. 


\section{Dedication}

Dedicated to my parents, soil ecologists everywhere, and students who have worked hard to achieve their dreams 


\section{Acknowledgments}

I am grateful for the many people who helped me over the course of this dissertation project and for the funding agencies that supported this research. I would like to thank Carol A. Miles, Mike Beug, Pat Labine, and my colleagues in the Ecological Agriculture program at The Evergreen State College for introducing me to the science of sustainable agriculture and for inspiring me to pursue this dissertation work. At Portland State University (PSU), I have benefited tremendously from the wonderful people on my committee who have guided me, nurtured me, challenged me, and advised me through this entire process. Mitch Cruzan, my primary advisor and the fearless leader of our lab has taught me more than I could ever list here. He has been my greatest cheerleader and invited me into his lab when I was a new student with just an idea about investigating the nontarget effects of genetically modified crops on soil organisms. Without him, this project may never have gotten off the ground. Lisa Weasel has been an inspiration to me in the academic realm and I am grateful for her valuable perspective on genetically modified crops which has helped to shape my research questions. Todd Rosenstiel has a vast breadth of knowledge and always provides insightful and thought-provoking comments on my work that I have been able to learn and grow from. Jim Bever is another member of my committee who I have learned so much from. He adopted me into his mycorrhizal lab at Indiana University where I gained skills in statistical analysis, bioinformatics, and molecular biology, and his lab is also the place where I've formed some of my strongest friendships. I am so grateful for his advice, guidance, and friendly 
manner that has helped guide me through the last few years of my graduate career. Daniel Ballhorn has provided me with valuable perspectives on tri-trophic interactions in a variety of plant species and I learned a lot from his chemical ecology reading group. Hap Pritchard, a former member of my committee, provided assistance with the design of this project in its early stages. I am also thankful for all the members of the Cruzan lab who have helped me with this project over the years including Kristin Anton, Paul Sochacki, Heather Machado, Trieste Musial, Alisa Ramakrishnan, Gina Marchini, Laura Taylor, Kevin Weitemier, Caitlin Lee, and Rachael Workman as well as fellow graduate students Melissa DeYoung, Sara Viernum, and Geoff Diemer. I have also benefited from the help of many amazing undergraduates, post-baccs, high school students, and volunteers in the lab including the "myco-boys" Brian Pace, Corey Guidry, and Matt LaPlante, as well as Luke Reyes, Hayley Darby, Danielle Butler, Belma Hergic, Scott Kiel, Dan Kowalkiewicz, Courtney Crawford, Kiernan Garrett, Alessandra Elliott, Erik Hasenkopf, Ann Rasmussen, Jennifer Jones, Hilary Miller, Sarah Arteaga, Sarah Gabriel, Madeline Steele, Melia Chase, Sage Wagner, Emily Fielding, and many others who helped with corn harvesting, processing, and slide-making during those long, hot days of summer. I would also like to thank the helpful people in the PSU Department of Biology, especially members of the biology office Leah Tuor, Autumn Droste, Sinnamon Tierney, and Amy Jolsted, the prep room staff Jane Boone and Lindsay Henderson, as well as the greenhouse managers Lane Greer and Linda Taylor. At Indiana University, I received research training, advice, comments on my book chapter, and help with statistical 
analysis from Jim Bever, Peggy Schultz, Ursel Schuette, Wittaya Kaonongbua, Jeremiah Henning, Liz Koziol, Tom Platt, Anna Rosling, Rich Phillips, Chris Hemmerich, and Wesley Beaulieu. Joe Morton at INVAM helped me identify mycorrhizal spores from my field site and has always been a great resource for all things mycorrhizae. Bill Landesman, Nancy C. Johnson, David Coleman, Diana Wall, and other members of the Ecological Society of America Soil Ecology Section helped me put together the 2009 ESA symposium from which the book I edited, Microbial Ecology in Sustainable Agroecosystems, was derived. I would also like to thank my family -- Mom, Dad, Robert, Ryan, and Clarke, and my friends Kelly Owen, Chris Dean, Cassandra Hoy, and Halo for assisting me with field work and for helping to processing root samples when it was late, hot, and we still had hundreds of plants to go. I would also like to thank my wonderful partner Neal for all of his love, patience, and support during my graduate studies and for helping me process root samples, even on the weekends and after he'd already worked a long day at his own job. Finally, I'd like to acknowledge my funding sources, without which I never could have done this work. Funding for this research was provided by the PSU Forbes Lea Foundation, PSU Scholarly and Creative grants to Brian Pace and Matt LaPlante for their work on this project, the Charles A. and Anne Morrow Lindbergh Foundation, Environmental Protection Agency Science to Achieve Results Fellowship, Botanical Society of America, Sigma Xi, The National Science Foundation, Sigma Delta Epsilon-Graduate Women in Science, and travel grants from The Ecological Society of America, PSU Biology Graduate Student Association, Botanical Society of America, 
Marie Brown Travel Grant, and a Miller Sustainability Travel Award. 


\section{Table of Contents}

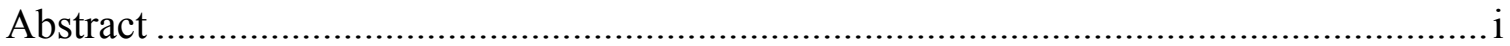

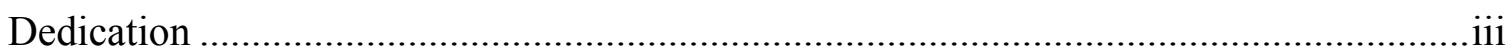

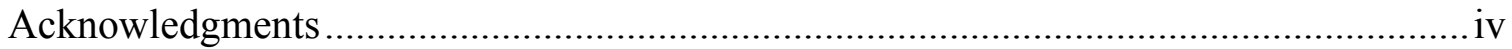

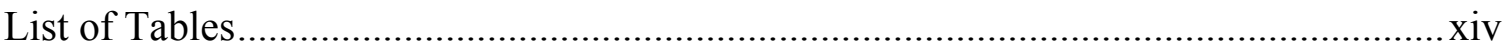

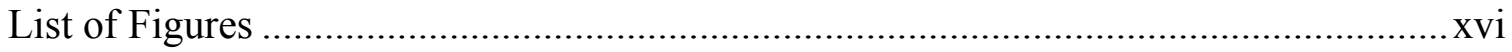

Chapter 1. Effects of the cultivation of genetically modified Bt crops on nontarget soil



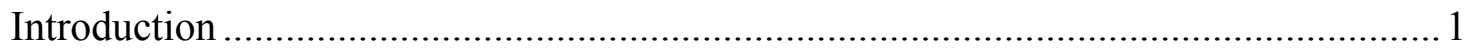

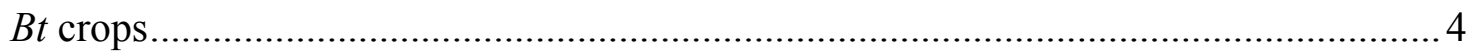

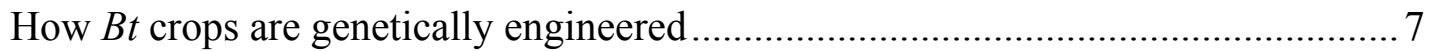

How $B t$ and other GM plants are regulated in the USA .................................... 8

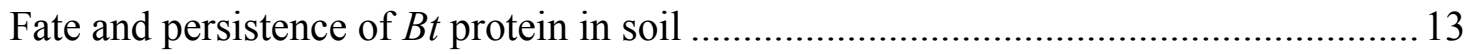

Effects of the cultivation of $B t$ plants on nontarget soil organisms ............................ 14

Effects of the cultivation of $B t$ crops on soil bacteria ....................................... 15

Effects of the cultivation of $B t$ crops on soil fungi .............................................. 27

Effects of the cultivation of $B t$ crops on protozoa and nematodes.......................... 37

Effects of the cultivation of $B t$ crops on earthworms .......................................... 42

Effects of the cultivation of $B t$ crops on microarthropods ....................................46

Effects of the cultivation of $B t$ crops on nontarget Lepidopteran larvae and aquatic

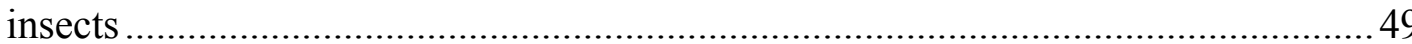




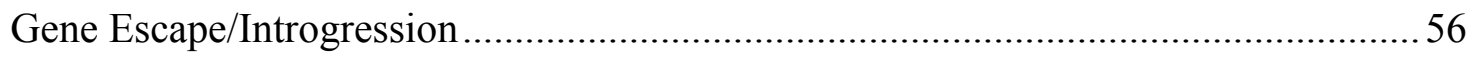

Can $B t$ crops have a role in sustainable agroecosystems?...........................................6 60

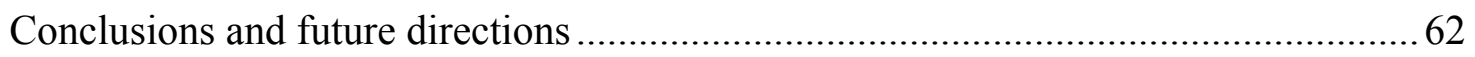

Chapter 2: The influence of fertilizer level and spore density on arbuscular mycorrhizal colonization of transgenic Bt 11 maize (Zea mays) in experimental microcosms ............65

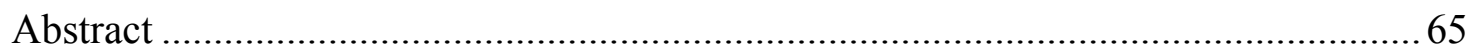

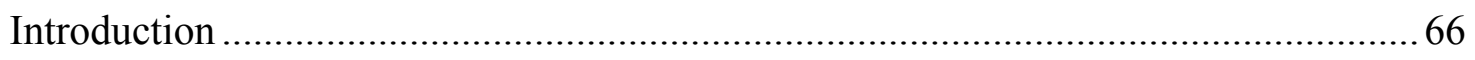

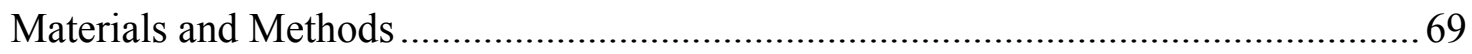

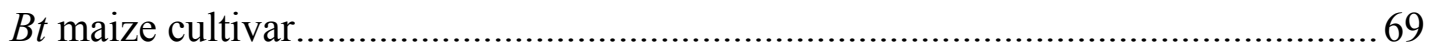

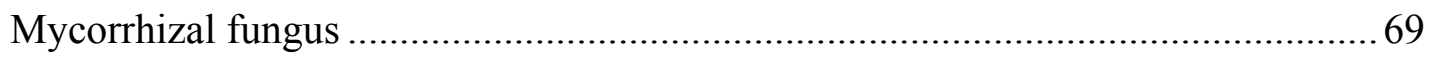

Plant cultivation and spore application ................................................................. 70

Growth conditions and fertilizer treatments....................................................... 70

Mycorrhizal fungus colonization assessment.......................................................... 71

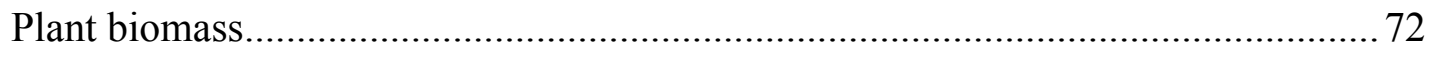

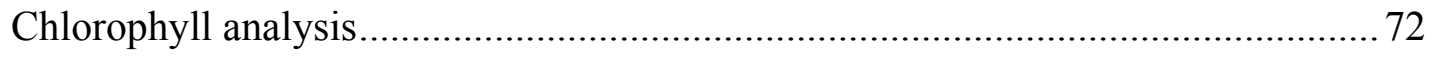

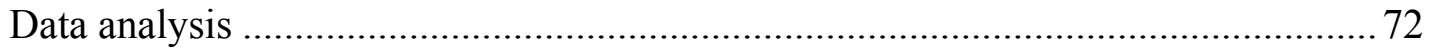

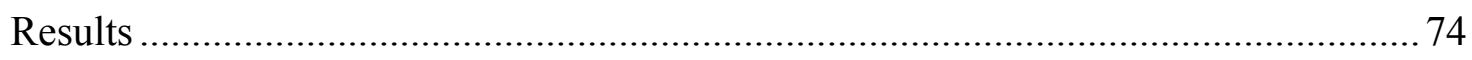

Effects of cultivar, fertilizer level, and spore inoculation level on AMF colonization

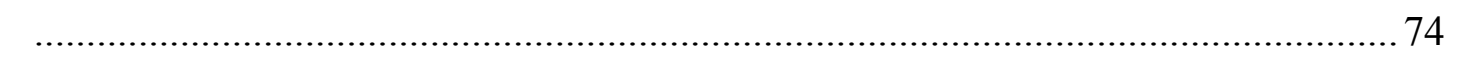

Effects of fertilizer level, cultivar, and spore inoculation level on plant growth .......75

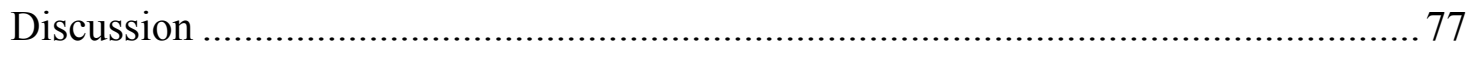


Chapter 3. Evidence of reduced arbuscular mycorrhizal fungal colonization in multiple

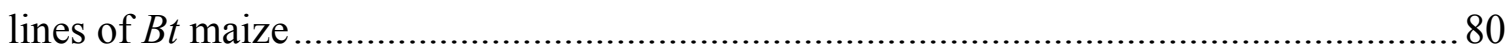

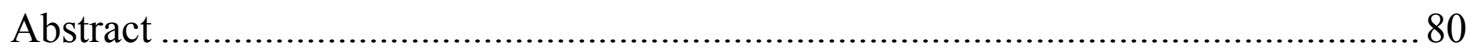

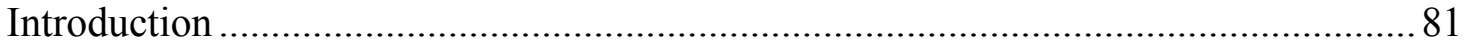

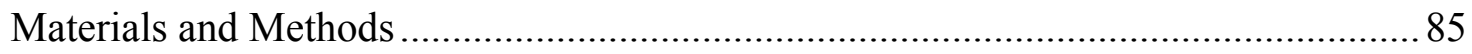

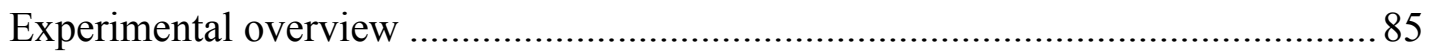

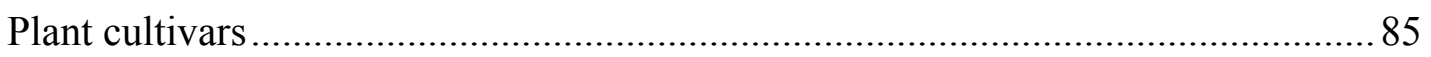

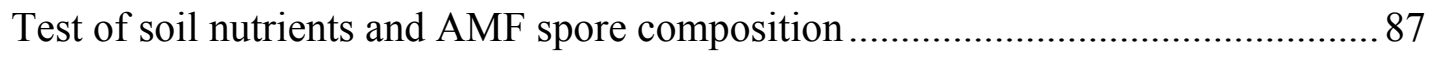

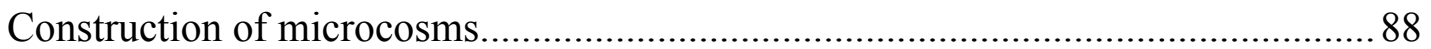

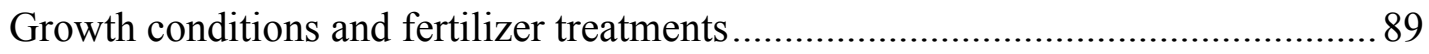

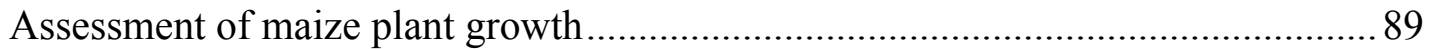

Test of $B t$ pre-conditioned soil on AMF colonization in G. max ........................... 89

Mycorrhizal fungus colonization assessment....................................................... 90

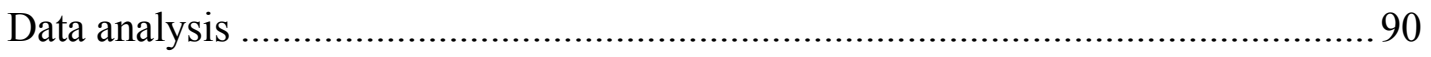

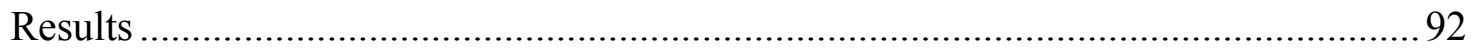

Effect of maize cultivar on AMF colonization................................................... 92

Effect of AMF colonization and cultivar type on maize growth...........................93

Effect of type of Cry protein expressed on AMF colonization in Bt maize..............93

Effect of soil pre-conditioned with $B t$ or P maize on AMF colonization, plant

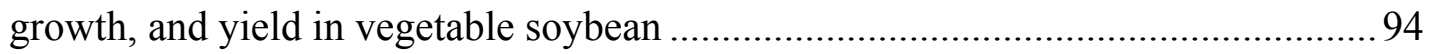

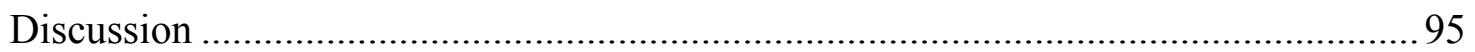


Chapter 4: Field evaluation of arbuscular mycorrhizal fungal colonization in Bacillus thuringiensis toxin-expressing $(B t)$ and non- $B t$ maize

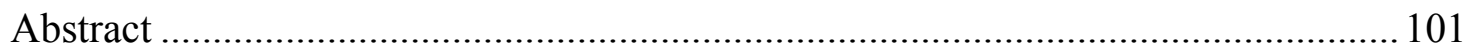

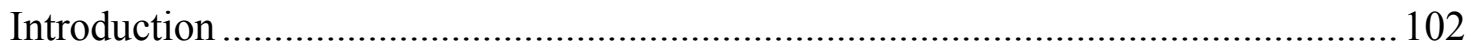

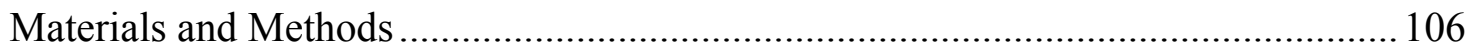

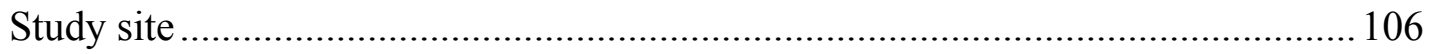

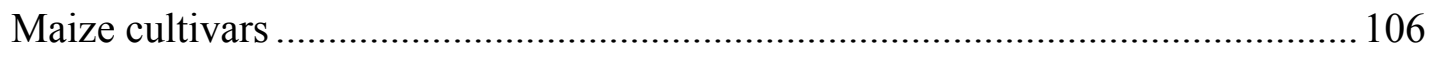

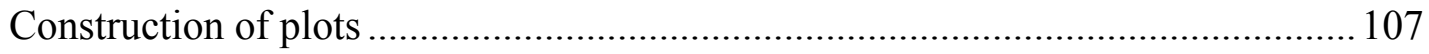

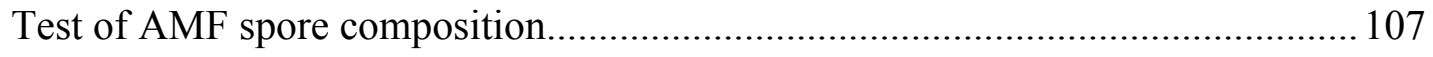

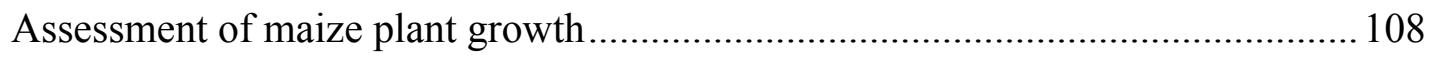

Mycorrhizal fungus colonization assessment................................................ 109

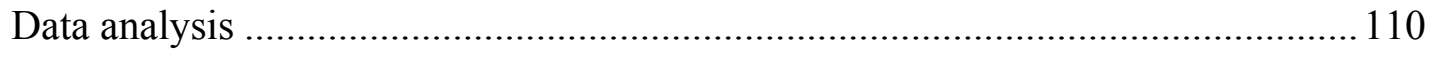

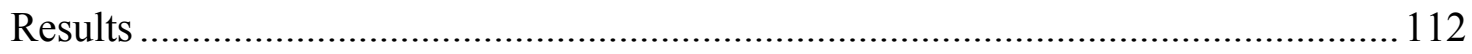

Effect of $B t$ maize on spore abundance and diversity .................................... 112

Effect of $B t$ maize on AMF colonization .......................................................... 113

Effect of AMF colonization and cultivar on maize growth ................................ 113

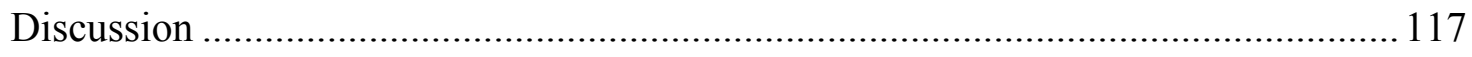

Chapter 5: Effect of $B t$ maize cultivation history on arbuscular mycorrhizal fungal colonization, spore abundance and diversity, and plant growth ................................ 123

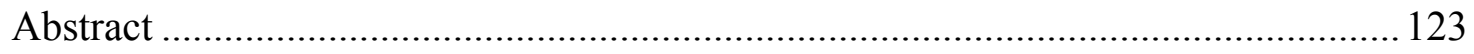

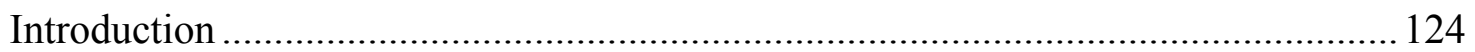




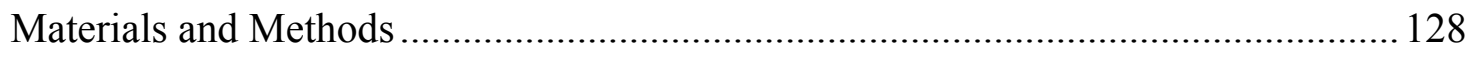

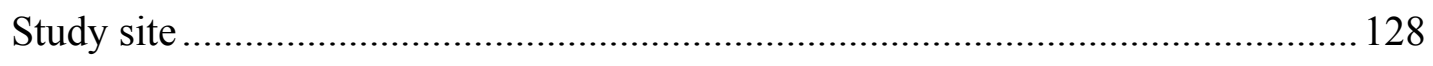

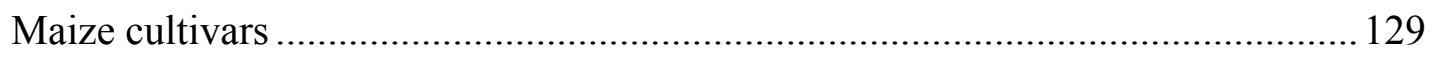

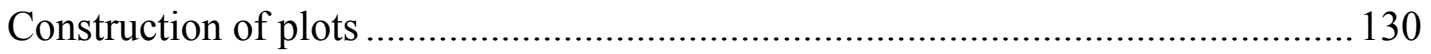

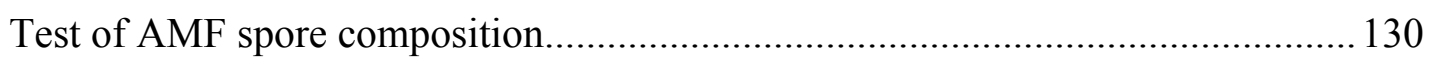

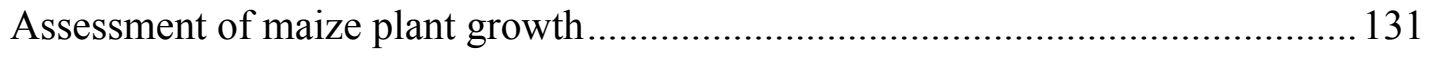

Mycorrhizal fungus colonization assessment.......................................................... 131

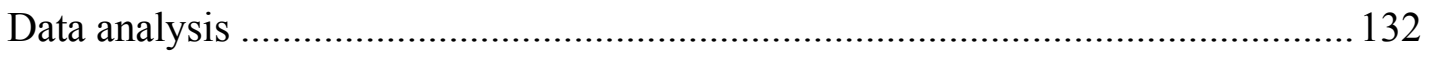

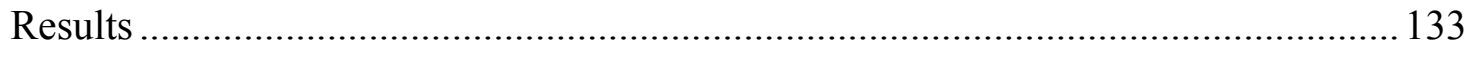

Effect of plot history on spore abundance and diversity ........................................ 133

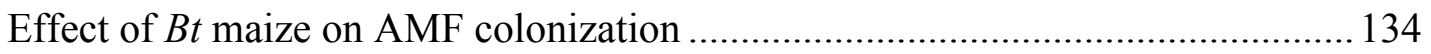

Effect of AMF colonization and cultivar on maize growth .................................... 134

Effect of plot history on AMF colonization and plant growth .................................135

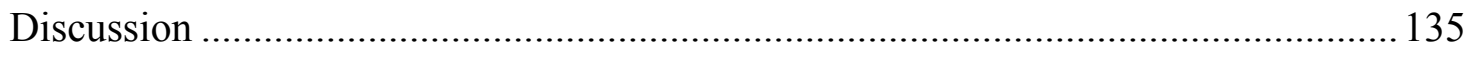

Chapter 6. Exploring potential mechanisms for lower AMF colonization in $B t$ maize.. 140

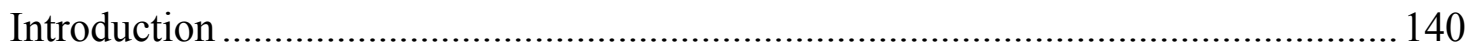

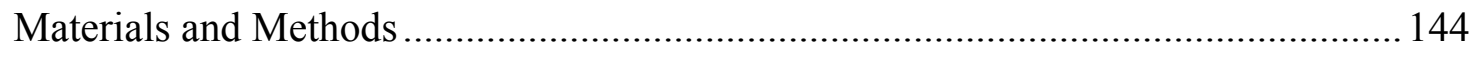

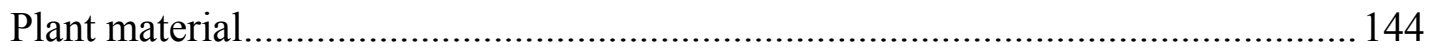

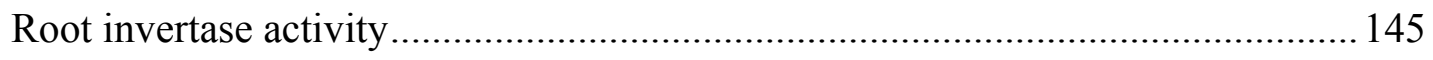

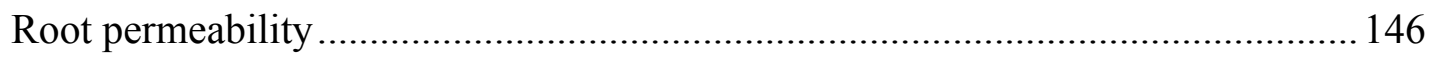

Aborted infection pegs, appressoria, and passage cells ........................................ 147 
Results

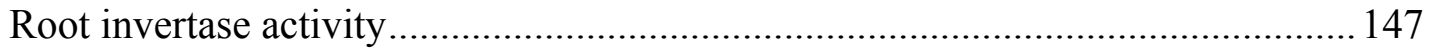

Root permeability: Lignin and suberin content in roots......................................... 148

Pre-symbiotic barriers: Aborted infection pegs, appressoria, and passage cells..... 148

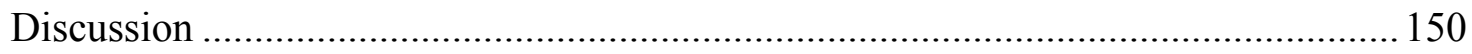

Chapter 7. Conclusions, future directions, and broader impacts..................................... 154

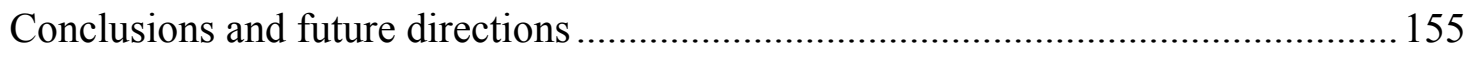

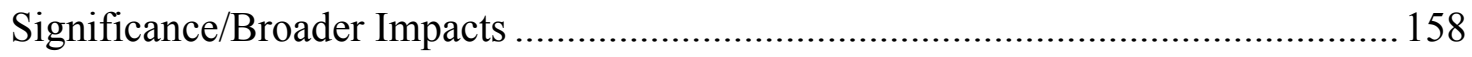

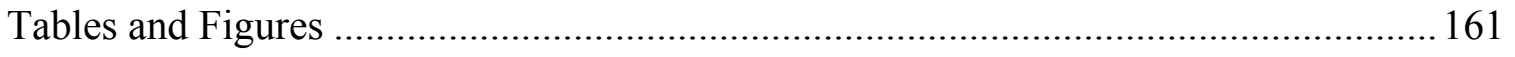

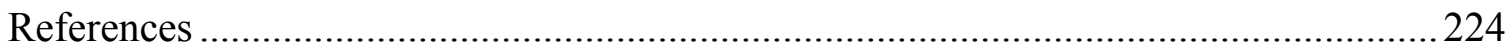

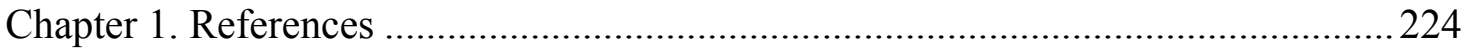

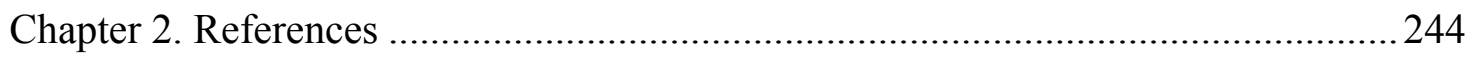

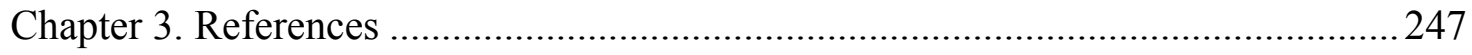

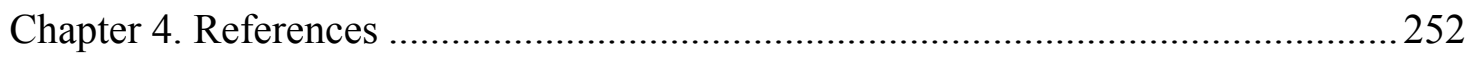

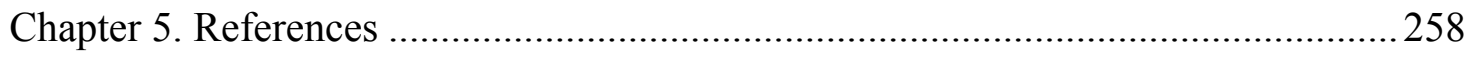

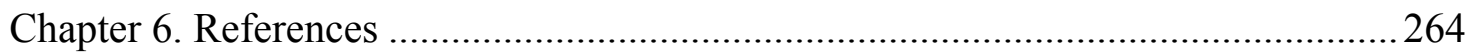

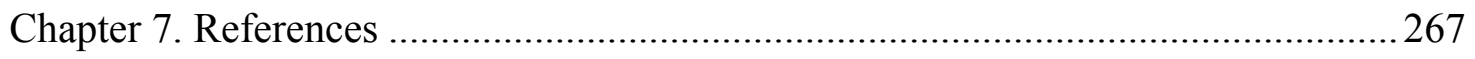




\section{List of Tables}

Table 1. Global area of genetically modified crops in 2010: By country (million ha) 161 Table 2. Adoption of genetically-modified (GM) plant varieties by major crop (maize, upland cotton, and soybean) in the United States, 2000-2011.

Table 3. Effects of cultivation of Bt crops and/or amendment with $B t$ proteins on soil bacteria

Table 4. Effects of cultivation of $B t$ crops and/or amendment with $B t$ proteins on soil fungi

Table 5. Effects of the cultivation of $B t$ crops and/or amendment with $B t$ protein on protozoa

Table 6. Effects of the cultivation of $B t$ crops and/or amendment with $B t$ protein on nematodes

Table 7. Effects of the cultivation of $B t$ crops and/or amendment with $B t$ protein on earthworms

Table 8. Effects of cultivation with $B t$ crops and/or amendment with $B t$ proteins on microarthropods

Table 9. Effects of the cultivation of Bt crops and/or amendment with $B t$ protein on selected non-target Lepidopteran and aquatic insect larvae.

Table 10. Effect of cultivar, fertilizer, and spore density on hyphae, arbuscules, and total AMF. 
Table 11. Effect of cultivar, fertilizer, and AMF on growth responses of $B t$ and non-Bt

maize.

Table 12. Effect of cultivar, fertilizer, and spore density on growth responses of $B t$ and non-Bt maize.

Table 13. $B t$ and non- $B t$ maize seeds used in greenhouse and field experiments.

Table 14. Effect of Bt protein on hyphae, arbuscules, vesicles, and total AMF.

Table 15. Effect of plant type (Bt or P) on AMF 60, 100, and $130 \mathrm{~d}$ after sowing. 201

Table 16. Effect of AMF on growth response of $B t$ and P maize at 60, 90, and $130 \mathrm{~d} .202$

Table 17. Effect of plant type of growth response 60, 90, and $130 \mathrm{~d}$ after sowing. $\quad 203$

Table 18. Tissue-specific stains used to detect lignin and suberin in cross-sections of $B t$

11 (Attribute) maize (sweetcorn) and its parental isoline (Providence). 


\section{List of Figures}

Figure 1. Effect of plant type, spore density, and fertilizer on AMF colonization. 205

Figure 2. Effect of fertilizer and spore density on AMF colonization in $B t$ and non- $B t$

maize.

Figure 3. Effect of fertilizer treatment and plant type (Bt or P) on plant growth.

Figure 4. Effect of fertilizer treatment, spore density, and plant type on growth. 208

Figure 5. Effect of fertilizer, spore density, and plant type on leaf chlorophyll. $\quad 210$

Figure 6. Effect of plant type (Bt or P) on AMF in a 60 day greenhouse study. 211

Figure 7. Effect of plant type (Bt or P) on AMF in a 100 day greenhouse study. $\quad 212$

Figure 8. Effect of plant type (Bt or P) on spore density in field plots.

Figure 9. Percent AMF colonization in $B t$ and $\mathrm{P}$ maize in a field experiment.

Figure 10. Correlation between leaf chlorophyll content and AMF at $130 \mathrm{~d}$.

Figure 11. Biomass of $B t$ and P maize 60, 90, and 130 days after sowing in a field. 216

Figure 12. Spore density in $B t$ and $\mathrm{P}$ plots as affected by cultivation history.

Figure 13. AMF colonization in Bt and P maize in split-plots in a field experiment. 218

Figure 14. Positive feedback fitness effects as affected by plot cultivation history. $\quad 219$

Figure 15. Apoplastic invertase activity in Bt 11 and P maize roots. 220

Figure 16. Stained cross-sections of $B t$ and non-Bt maize roots. 222

Figure 17. Potential pre-symboiotic barriers in $B t 11$ and non-Bt maize. 


\section{Chapter 1. Effects of the cultivation of genetically modified $B t$ crops on nontarget soil organisms}

Published as: Cheeke, T. E. Effects of the cultivation of genetically modified Bt crops on nontarget soil organisms. In: Microbial Ecology in Sustainable Agroecosystems. Advances in Agroecology Series. Cheeke, T.E., Coleman, D.C., Wall, D.H. (Eds.). Boca Raton: CRC Press. Pp. 153-227.

\section{Introduction}

Genetically modified (GM) crops were first commercially introduced in 1996 and are now cultivated in at least 25 countries (Table 1; James, 2010). Historically, the primary types of GM crops cultivated were herbicide-tolerant (i.e., Roundup Ready® soybean) and insect-resistant (genetically engineered to express a gene from Bacillus thuringiensis, i.e., $B t$ maize), but more recently, new GM cultivars have been developed that offer stacked traits (herbicide tolerance plus resistance to multiple insect pests); increased stress tolerance (e.g., salt stress or drought tolerant varieties); improved nutrient status; physiological enhancements (longer storage, delayed ripening); and even pharmaceutical crops engineered to produce drugs, human growth hormones, and other products of medical interest (Stotzky and Saxena, 2009). Since the commercial introduction of GM plants, the acreage dedicated to GM crop cultivation has increased each year, such that the majority of all major crop plants grown in the United States --soybean, cotton, and maize -- are genetically engineered (USDA, 2010). Developing countries also continue to increase their share of global GM crop production and now account for almost half (46\%) 
of the global hectarage of GM crops (James, 2010). This rapid and widespread adoption of GM crops has led to a dramatic shift in the agricultural landscape since the mid-1990s and has raised questions about the impact of agricultural biotechnology on nontarget organisms in the soil environment.

Although some GM crops can provide a variety of agricultural benefits, there may also be potential risks to nontarget organisms. Some of the key scientific concerns regarding the widespread cultivation of GM crops include (1) the potential for gene flow from transgenic plants to related species (e.g., Mercer and Wainwright, 2008; PineyroNelson et al., 2009); (2) persistence of GM plant material in the environment (e.g., Saxena and Stotzky, 2001b; Zwahlen et al., 2003a; Stotzky, 2004; Flores et al., 2005; Tarkalson et al., 2008); (3) the evolution of pest resistance (e.g., Gould et al., 2002; Abel and Adamczyk, 2004; Huang et al., 2007; Gao et al., 2010); (4) risks to the environment associated with changes in the agricultural landscape or farming practices associated with the adoption of GM crops (e.g., Krogh et al., 2007; Lupwayi et al., 2007; Watrud et al., 2011); and (5) the risk to nontarget organisms, including mammals, birds, fish, insects, and soil organisms (e.g., Stotzky, 2000; Adamczyk and Hardee, 2002; Kowalchuk et al., 2003; Clark et al., 2005; Rosi-Marshall et al., 2007; Thies and Devare, 2007; Icoz and Stotzky, 2008b; Lang and Otto, 2010; Then, 2010; Gatehouse et al., 2011).

This chapter summarizes the environmental risk assessment research of $B t$ crops to date in soil and offers suggestions on how to examine and understand better the effects of 
these types of GM crops on soil organisms. This chapter includes discussion of the following: an introduction to $B t$ crops (what they are, where they are grown, how they are engineered, etc.); how $B t$ and other transgenic crops are regulated in the United States; the fate of $B t$ plant material in soil (how $B t$ toxin enters soil, binding properties, potential differences in degradation rates of transgenic plant material, etc.); effects of $B t$ crops on soil organisms, including bacteria, fungi, protozoa, nematodes, and soil invertebrates (earthworms, microarthropods, insects); and a brief discussion of the escape and introgression of transgenes as this could also have nontarget effects on soil organisms. The question of whether or not Bt crops can contribute to the sustainability of agroecosystems is also discussed. The chapter concludes with a summary and recommendations for future research directions. The information herein follows many other excellent reviews evaluating effects of transgenic crop cultivation on nontarget organisms in the soil environment (e.g., Stotzky, 2000, 2002; Giovannetti, 2003; Kowalchuk et al., 2003; Saxena and Stotzky, 2003; Motavalli et al., 2004; Stotzky, 2004; Giovannetti et al., 2005; Liu et al., 2005; O'Callaghan et al., 2005; Thies and Devare, 2007; Icoz and Stotzky, 2008b; Liu, 2010; Saxena et al., 2010). Although herbicidetolerance is often incorporated into $B t$ cultivars as a stacked-trait, nontarget effects of GM herbicide-tolerant crops on soil organisms are not included in this review because the genetic insertion has been shown to have no direct effect on soil organisms (although the use of this technology may have indirect effects on soil organisms as a result of changes 
in agricultural practices) (e.g., Siciliano and Germida, 1999; Dunfield and Germida, 2003; Kowalchuk et al., 2003; Dunfield and Germida, 2004; Krogh et al., 2007; Griffiths et al., 2008; reviewed by Lundgren et al., 2009; Watrud et al., 2011).

\section{Bt crops}

Insect-resistant crops are genetically engineered to express insecticidal toxins derived from the spore-forming soil bacterium Bacillus thuringiensis (Bt). Naturally occurring $B t$ soil organisms produce insecticidal crystalline proteins (called Cry proteins) during sporulation that are toxic to the larvae of certain insects (Hofte and Whiteley, 1989; reviewed in Schnepf et al., 1998; USDA, 2010). To date, more than 60 different Cry proteins have been identified that exhibit a high degree of specificity toward Lepidoptera (e.g., moths and butterflies), Coleoptera (e.g., beetles), Diptera (e.g., flies and mosquitoes), Homoptera (e.g., cicadas, leafhoppers, aphids, scales), Hymenoptera (e.g., wasps, bees, ants, sawflies), Orthoptera (e.g., grasshoppers, crickets and locusts), Mallophaga (e.g., lice), and nematodes, (reviewed in Schnepf et al., 1998; Federici, 2002; Stotzky, 2002; Lee et al., 2003; Icoz and Stotzky, 2008b; Sanchis, 2011). Some Bt proteins have even been used for targeted treatment of some types of cancer cells in humans (e.g., Ito et al., 2004; Yamashita et al., 2005; Ohba et al., 2009; Tharakan et al., 2009; Nagamatsu et al., 2010; Poornima et al., 2010; Wong et al., 2010).

Formulations of $B t$ carrying the parasporal crystals have been used as a natural insecticide in agricultural systems since the 1930s (Hofte and Whiteley, 1989; reviewed 
in Beegle and Yamamoto, 1992; Sanchis, 2011), but success is often compromised by the poor survival of the natural form of $B t$ in the environment (Griego and Spence, 1978; West, 1984; West et al., 1985; Clark et al., 2005). Moreover, the Bt toxin present in the soil bacteria is not activated until cleaved by alkaline hydrolysis in the gut of a susceptible insect larva (Hofte and Whiteley, 1989) and activation may also require the presence of indigenous bacteria in the midgut of susceptible insects (Broderick et al., 2006; Broderick et al., 2009; reviewed by Then, 2010). The Bt gene that is genetically engineered into plants, however, is truncated, and constitutively produces only the preactivated Cry protein in the cells of the genetically modified plant (e.g., Shu et al., 2002; Xu et al., 2006).

At the present time, the two major crops that contain genes coding for insecticidal $B t$ toxin are $B t$ maize and $B t$ cotton. Other $B t$ crops that have been developed include $B t$ potato, $B t$ tobacco, $B t$ spruce, $B t$ tomato, $B t$ rice, $B t$ eggplant, $B t$ sunflower, and $B t$ canola, although not all of these are presently commercially available. In $2010,86 \%$ of the maize and $93 \%$ of the cotton cultivated in the USA was genetically modified to express herbicide tolerance, insect resistance, or some combination of inserted traits (USDA, 2010; Table 2), making up $26 \%$ and $49 \%$ of the global GM crop acreage, respectively (James, 2010). The dramatic rise in the adoption rate of GM crops resulted primarily from the development of GM varieties containing "stacked traits" or "pyramided traits" (as opposed to single traits in one variety or hybrid). The term "stacked trait" refers to a 
plant that has been engineered to express multiple toxins against different pests (e.g., protection against European corn borer and corn root worm) or contains multiple plant protection properties (e.g., herbicide tolerance + insect resistance), whereas a "pyramided trait" is one in which multiple toxins are expressed to target the same pest (EPA, 2011). In $2009,75 \%$ of the genetically modified maize hybrids in the USA were engineered with double or triple stacked traits (James, 2010). One of the newest GM maize hybrids, SmartStax ${ }^{\mathrm{TM}}$, was engineered to express eight different genes coding for pest resistance and herbicide tolerance and produces six different types of Cry proteins--Cry1A.105, Cry2Ab2, Cry1F, Cry3Bb1, Cry34Ab1, and Cry35Ab1 to protect plants against 13 different insect pests (European corn borer, Southwestern corn borer, Southern cornstalk borer, corn earworm, fall armyworm, stalk borer, lesser corn stalk borer, sugarcane borer, Western bean cutworm, black cutworm, Western com rootworm, Northern corn rootworm, Mexican corn rootworm) (EPA, 2009). Future GM crop varieties are expected to include multiple traits for pest resistance and tolerance to herbicides and drought, as well as nutritional traits, such as high omega-3 oil in soybean or enhanced nutrient content of other staple crops (James, 2010; Monsanto, 2011). Stacked and pyramided traits have become a very important feature of GM crops and will continue to be adopted by farmers worldwide. 
How Bt crops are genetically engineered

Genetically modified plants can be engineered to express a variety of novel traits (usually with DNA obtained from another type of organism) that confer protection against insect pests, tolerance to herbicides, increase vitamin content or nutrient status, improve drought tolerance, produce pharmaceuticals, or impart almost any other characteristic that is deemed to be agriculturally or commercially important. The process of genetic engineering involves the identification and isolation of desired genes (e.g., the genes in strains of the soil bacterium, B. thuringiensis, that produce $B t$ proteins), the selection of a host plant (often called the parental line or the parental isoline), insertion of the foreign gene into the host plant cells (called transformation, often using a bacterial vector, such as Agrobacterium tumefaciens, electroporation, or microprojectile bombardment to insert physically the foreign DNA into the host cell), screening for successful transformants, and then regeneration of the whole transformed plants. The individual transformation events are indicated in the nomenclature of the GM plant, e.g., $B t$ corn Event 11 (often abbreviated to $B t 11$ or Event $B t 11$ ). The non-transgenic parental cultivar from which the transgenic line was engineered is called the non-transgenic isoline. The isoline is a plant line that is nearly genetically identical to its parental basehybrid except for the genetic insertion(s) - e.g., Bt 11 and its non-transgenic parental isoline, Providence.

Although it is usually clear through selective screening which plant cells have taken 
up the foreign genes, it is seldom known exactly where in the host plant genome new DNA has been incorporated. Consequently, unintended pleiotropic effects as a result of the genetic insertion can occur (e.g., Sheveleva et al., 1998; reviewed in Wang et al., 2003; reviewed in Giovannetti et al., 2005), and whole transgenic plants must be carefully evaluated for plant performance and undesirable phenotypic characteristics before advancing to the GM crop registration stage (see How Bt and other GM plants are regulated in the USA). While obviously abnormal phenotypes are eliminated during this screening process, it is possible that certain types of pleiotropic effects, such as those that that influence the physiology (e.g., sugar allocation, enzyme activity in roots, lignin content) of individual insertion events may not be detected in some plant lines.

\section{How Bt and other GM plants are regulated in the USA}

The United States of America is the world's largest producer of genetically modified crops, with 66.8 million hectares cultivated with GM crops in 2010 , more than twice the biotech cultivation area of any other country (Table 1). The United States government oversees the development, deployment, and safety of transgenic organisms through three separate agencies; The United States Department of Agriculture, Animal and Plant Health Inspection Service (USDA-APHIS), The Environmental Protection Agency (EPA), and the Food and Drug Administration (FDA). Together, these government agencies review potential areas of concern regarding the introduction of new GM crops, including the 
potential for gene flow, resistance management, effects on nontarget organisms and the environment, and the introduction or elevation of potential allergens. The specific regulatory duties of each agency are as follows:

1. The USDA is responsible for regulating the introduction (importation, interstate transport, and field release) of GM organisms (plants, insects, microbes, etc.) or any other organism that is, or could be, a plant pest. The Biotechnology Regulatory Service (BRS) of APHIS is the regulatory body within the USDA responsible for deciding whether a genetically engineered organism is as safe for the environment as its traditionally bred counterpart.

2. The EPA regulates and assesses the environmental impact of certain types of GM organisms with pesticidal properties (e.g., insect-resistance, disease resistance, and certain plant growth regulator expressing products) through the Federal Insecticide, Fungicide, Rodenticide Act (FIFRA). The EPA regulates the gene and its product, not the plant (as does APHIS and FDA), as plant-incorporated protectants (PIPs). Biotechnology companies submit their data to the EPA for permitting and approval before these crops can be grown commercially. The data requirements for EPA approval include product characterization, mammalian toxicity, allergenicity potential, effects on nontarget organisms, environmental fate, and for Bt products, insect resistance management strategies (EPA, 2010). EPA also sets tolerances under the Federal Food Drug and Cosmetic Act 
(FFDCA) for residues of pesticidal substances in or on food and feed crops like $B t$ maize or cotton (personal communication, Wozniak, 2011).

3. The FDA examines the toxicity and allergenicity of GM plants under the FFDCA. This includes oversight of food plants that contain transgenic proteins that are not normally found in that food source (e.g., an animal gene expressed in plants). When the FDA conducts safety evaluations of GM crops, it evaluates both the final product and the techniques used to develop them. The FDA's process, however, is voluntary; the FDA's approach is a comparative one based on a compositional analysis of the GM plant and its non-GM counterpart (personal communication, Wozniak, 2011). The FDA also enforces the tolerances set by the EPA in the event tolerance limits are exceeded.

More information about the regulatory responsibilities of each of these US government agencies is available at http://usbiotechreg.nbii.gov/.

It should be noted and emphasized here that the US regulatory agencies do not generally conduct risk assessment studies themselves; the regulatory agencies review the data that biotechnology companies provide (which is often conducted by independent third party laboratories), and in order to get a new GM plant approved for commercial use, the product must show minimal risk to nontarget organisms, human health, and the environment. It takes many years (usually $>6$ ) for seed companies to go from the discovery phase to commercial sales and distribution (Monsanto, 2011). Usually, early 
contact is made with the regulatory agencies and a reiterative dialogue between the biotechnology company and the regulatory agencies takes place early in the registration process (personal communication, Wozniak, 2011). Data are then submitted to the EPA PIP products division for an experimental use permit a few years before registration occurs (personal communication, Wozniak, 2011). Once a new crop line advances to the pre-launch phase, there is a $90 \%$ chance that it will be introduced into the commercial marketplace (Monsanto, 2011). In this final phase of development, the regulatory data are submitted to the EPA, large quantities of seeds are generated, and the premarket advertising campaign begins. After health and safety data have passed EPA guidelines, the product is approved for commercial sales, and the product is launched.

Some types of GM crops that receive EPA approval have restrictions that limit how and where a particular crop can be grown. For example, in order to plant $B t$ crops, farmers are required to keep 5 to $20 \%$ of their land (depending on the $B t$ product in use) in a non- $B t$ refuge to help minimize the rate of resistance of targeted pests (EPA, 2010). The EPA also monitors the potential for gene flow between GM plants and wild-type relatives and generally does not approve GM crops that have wild-type relatives that grow in close-proximity to GM crop regions. For the major GM crops that have been developed thus far (corn, soy, canola, cotton), there is little risk for cross-pollination in the United States, as the wild relatives of most of these crops are found primarily in tropical areas. In the case of $B t$ cotton, however, where the potential for gene flow to wild 
cotton relatives does exist in the USA (i.e., Hawaii, Florida - south of State Route 60, Puerto Rico, and the U.S. Virgin islands), the EPA has restricted sales and distribution of $B t$ cotton within these areas (EPA, 2010). However, even when there is little chance for gene flow between transgenic and feral or indigenous sexually compatible wild relatives (SCWR) in the United States, there is a real possibility of gene flow between GM and wild-type varieties in other regions of the world. For example, gene flow has been detected between $B t$ maize and native maize landraces in Mexico (Quist and Chapela, 2001; Pineyro-Nelson et al., 2009), and despite limits on where Bt cotton can be cultivated in the US, Bt cotton is also grown in at least 12 other countries, several of which are in tropical regions associated with areas which are within the center of origin for New World cotton species (Table 1).

As more and different types of GM crops are developed, the likelihood of gene transfer between SCWR and GM crops is expected to increase, especially if grown in regions where the level of government oversight is not as extensive as it is in the USA. Thus, while the safety testing and approval process can minimize much of the environmental and health/safety risk of GM crops in the United States, it is still possible for some GM crops to have nontarget effects in the environment or on organisms that have either not been tested or may have unexpected effects under certain environmental conditions. It is, therefore, important that GM crops continue to be evaluated for nontarget effects under a variety of environmental and experimental scenarios, even after 
they have been approved for commercial use in the USA.

\section{Fate and persistence of Bt protein in soil}

Numerous studies have shown that insecticidal $B t$ proteins are released from transgenic plants into soil through root exudates (e.g., Saxena et al., 1999; Saxena and Stotzky, 2000; Saxena and Stotzky, 2001a; Saxena et al., 2002a; Saxena et al., 2002b; Saxena et al., 2004; Stotzky, 2004; Icoz and Stotzky, 2008a; Li et al., 2009), pollen (Losey et al., 1999; Zangerl et al., 2001), and plant residue decomposition (Zwahlen et al., 2003a). Once in soil, the $B t$ toxins bind to clay particles (Tapp et al., 1994; Tapp and Stotzky, 1995) and humic acids (Crecchio and Stotzky, 1998) and can retain their insecticidal properties for at least up to 234 days (Tapp and Stotzky, 1998). In laboratory studies, Cryl Ab protein from $B t$ maize root exudates persisted in soil for at least 180 days and for 350 days in soil amended with Bt maize plant material (Saxena and Stotzky, 2002). In a greenhouse pot study where one $B t$ cotton line, two stacked $B t$ and cowpea trypsin inhibitor $(B t+C p T I)$ cotton lines, and their non-GM isolines were consecutively cultivated for four years, Cry1Ac and CpTI proteins persisted in soil (Chen et al., 2011), supporting a previous study where $41 \%$ and $60 \%$ of the introduced amounts of $B t$ protein from stems and leaves of two $B t$ cottons (Events $B t-Z \mathrm{k}$ and $B t$-GK, respectively) incorporated into soil under laboratory conditions remained after 56 days (Sun et al., 2007). In field studies, Cryl Ab protein from transgenic maize litter has been shown to 
persist for at least 8 months (Zwahlen et al., 2003a), although $B t$ protein in soil does not appear to accumulate over time (e.g, Hopkins and Gregorich, 2003; Baumgarte and Tebbe, 2005; Icoz and Stotzky, 2008a).

A higher lignin content has been reported in some $B t$ crops, including several different lines of $B t$ maize (Saxena and Stotzky, 2001c; Flores et al., 2005; Poerschmann et al., 2005; Fang et al., 2007). Higher lignin content has also been reported for $B t$ tobacco, $B t$ cotton, $B t$ canola, $B t$ potato, and $B t$ rice, although these differences were not statistically significant when compared with the non- $B t$ isolines (Flores et al., 2005). The slower decomposition of $B t$ organic material in soil, in some cases, has been attributed to higher lignin in transgenic plant residues (Saxena and Stotzky, 2001c; Stotzky, 2004; Flores et al., 2005). As a result, soil organisms may have a longer exposure to the $B t$ toxins as they are slowly released from organic matter and soil particles over time (Zwahlen et al., 2003a; Stotzky, 2004).

\section{Effects of the cultivation of Bt plants on nontarget soil organisms}

Soil organisms, including bacteria, fungi, protozoa, nematodes, earthworms, and microarthropods, have a tremendously important role in maintaining plant health and soil fertility through the decomposition of organic matter, nutrient mineralization, providing protection against disease, and improving soil structure. Moreover, symbiotic soil organisms, such as nitrogen-fixing bacteria and arbuscular mycorrhizal fungi (AMF), 
provide nutritional benefits to plants in exchange for carbon resources and protection by the host plant. Although ubiquitous, many soil organisms are sensitive to a variety of agricultural practices, including pesticide applications, tilling, cultivation practices (e.g., monocultures versus intercropping, compost versus chemical fertilizer), and even the type of plant grown. Because of their close associations with plant roots, some soil organisms, such as AMF or nitrogen-fixing bacteria may be more sensitive to changes in the physiology of the host plant or in the composition of root exudates as a result of genetic engineering than their free-living counterparts in the soil.

\section{Effects of the cultivation of Bt crops on soil bacteria}

Bacteria are by far the most abundant organisms in soil and are important for nutrient mineralization, decomposition of organic matter, protection against plant pathogens, degradation of chemicals/toxins in the environment, and nutrient cycling. In both natural and agroecosystems, bacterial abundance is highest in the rhizosphere (the narrow area of soil directly surrounding and influenced by plant roots). Plants support the development of microbial communities in the rhizosphere by producing root exudates that contain carbon-rich nutrients such as carbohydrates and proteins (Grayston et al., 1996; Morgan et al., 2005). Soil organisms take advantage of these carbon resources and plants benefit via increased nutrient availability, improved mineral uptake, and enhanced soil fertility

provided by the soil microbial community (Smith and Gianinazzi-Pearson, 1988; Morgan 
et al., 2005; Smith and Read, 2008).

Because of their close association with plant roots and their importance to biotic soil processes, some of the earliest environmental risk assessment research was aimed at determining the nontarget effects of $B t$ crops on soil bacteria (summarized in Table 3). It was thought that a change in the composition or quality of root exudates from $B t$ crops and/or accumulation of $B t$ toxin in the rhizosphere might modify the composition, and/or activity of soil microbes, ultimately influencing biotically-driven ecosystem processes and affecting plant growth and health. To date, however, only a few studies have reported any significant effects (either positive or negative) on soil bacteria as a result of the addition of purified $B t$ proteins to soil, amendment of soil with $B t$ plant material, or cultivation with $B t$ crops (e.g., Donegan et al., 1995; Wu et al., 2004a; Wu et al., 2004b; Castaldini et al., 2005; Rui et al., 2005; Xue et al., 2005; Fang et al., 2007; Sun et al., 2007; Chen et al., 2011).In one of the first risk assessment studies to examine the effects of $B t$ plants on soil bacteria, Donegan et al. (1995) reported that soils amended with leaves of different lines of transgenic $B t$ cotton resulted in a statistically significant, but transient, increase in culturable bacteria. However, the plant line specific response and the lack of effects of the addition of purified Bt protein (Cry1Ac) on soil bacteria suggest that these effects may not have resulted from the Cry1Ac protein but, rather, from a pleiotropic effect (change in a single gene that affects multiple phenotypic traits) of the genetic manipulation. In a greenhouse study, Castaldini et al. (2005) observed differences 
in soil bacteria isolated from soil amended with $B t$ versus non- $B t$ maize residue, with members of a subgroup of the genus Bacillus isolated from soil amended with $B t$ biomass while growth-promoting rhizobacteria were isolated from soil amended with non- $B t$ maize biomass. When plant residues of $B t$ and non- $B t$ maize were kept mixed with soil for up to 4 months, soil respiration was reduced by $10 \%$ in the $B t$ maize treatments (Castaldini et al., 2005). Fang et al. (2007) reported that soil amended with $B t$ maize biomass had a significantly different microbial community structure than soil amended with non-Bt maize biomass as determined by substrate utilization profiles and denaturing gradient gel electrophoresis (DGGE) patterns, and linked the alterations in the structure of soil microbial communities to the higher lignin content detected in the $B t$ maize plants. In soil amended with $B t$ rice straw versus non- $B t$ rice straw, differences in biological activities (dehydrogenase activity, methanogenesis, hydrogen production, and anaerobic respiration) were detected, but there was no direct toxic effect of Cry $1 \mathrm{Ab}$ protein (Wu et al., 2004a; Wu et al., 2004b). In a decomposition study where litterbags containing $B t$ rice and non- $B t$ rice roots or $B t$ and non- $B t$ rice straw were buried in the field and sampled over two years, Wu et al. (2009) found that $B t$ rice roots decomposed relatively faster than non- $B t$ rice roots in the first 200 days, but found no difference in microbial decomposition rates between $B t$ and non- $B t$ rice straw as determined by changes in ashfree mass remaining and changes in total carbon and total nitrogen content after decomposition. The incorporation of $B t$ cotton stems and leaves into soil microcosms had 
a positive effect on soil urease, acid phosphomonoesterase, invertase, and cellulose activities but a negative effect on arylsulfatase activity (Sun et al., 2007). The addition of cotton tissue to soil most likely stimulated microbial activity (and thus influenced soil enzyme activity), potentially masking any negative effect of $B t$ protein on soil microbial and/or soil enzyme activity (Sun et al., 2007).

When soil was cultivated with $B t$ maize, Xue et al. (2005) reported a lower ratio of gram-positive to gram-negative bacteria compared to soil cultivated with non-Bt maize. In microcosm and greenhouse experiments, Castaldini et al. (2005) reported differences in rhizospheric eubacterial communities and in culturable rhizospheric heterotrophic bacteria in soil cultivated with $B t$ maize versus non- $B t$ maize as determined by DGGE analysis of $16 \mathrm{~S}$ rRNA genes and culturing methods. In soil cultivated with $B t$ and non-Bt cotton, Rui et al. (2005) found that the numbers of colony forming units (CFU) of three different bacterial functional groups (nitrogen fixing, inorganic phosphate dissolving, and potassium dissolving) were lower in soil collected from the rhizosphere of $B t$ cotton at the early and middle stages of plant growth than in soil cultivated with non-Bt cotton. However, addition of purified Cryl Ac protein directly to soil had no effect on the number of CFU of any of the three bacterial functional groups (except for a decrease in the nitrogen-fixing bacteria when concentrations of $B t$ protein were greater than $500 \mathrm{ng} / \mathrm{g}$ ) (Rui et al., 2005). In soil consecutively cultivated for four years with $B t$ cotton, $B t$ and cowpea trypsin inhibitor $(B t+C p T I)$ cotton, and non- $B t$ cotton lines, there was a decrease 
in microbial biomass carbon (MBC), soil microbial activity as determined by catalase activity and fluorescein diacetate hydrolysis, and some enzyme activities (nitrate reductase, acid phosphomonoesterase, arylsulfatase, $\beta$-glucosidase, and protease) in the $B t$ and $B t+C p T I$ cotton lines compared to soil cultivated with non- $B t$ cotton in greenhouse trials (Chen et al., 2011). The decreases in MBC, microbial activity, and enzyme activities were correlated with increasing CryAc protein content (Chen et al., 2011).

Most studies, however, have reported no negative effects of purified $B t$ proteins, $B t$ plant biomass, or the cultivation of $B t$ crops on soil bacteria (Donegan et al., 1995; Escher et al., 2000; Saxena and Stotzky, 2001a; Koskella and Stotzky, 2002; Ferreira et al., 2003; Blackwood and Buyer, 2004; Brusetti et al., 2004; Devare et al., 2004; Wu et al., 2004a; Wu et al., 2004b; Baumgarte and Tebbe, 2005; Fang et al., 2005; Flores et al., 2005; Griffiths et al., 2005; Rui et al., 2005; Griffiths et al., 2006; Shen et al., 2006; Devare et al., 2007; Griffiths et al., 2007b; Lamarche and Hamelin, 2007; Icoz and Stotzky, 2008a; Liu et al., 2008; Oliveira et al., 2008; Hu et al., 2009; Lu et al., 2010a; Lu et al., 2010b; Miethling-Graff et al., 2010; Tan et al., 2010; Li et al., 2011; Xue et al., 2011). When purified Bt proteins were added to soil, there was no effect of Cry1 Ab or Cry1Ac on culturable bacteria (Donegan et al., 1995), culturable inorganic phosphate dissolving or potassium dissolving bacteria (Rui et al., 2005), on the population size of culturable heterotrophic bacteria (Ferreira et al., 2003), or on microbial community 
structure as determined by phospholipid fatty acid (PLFA) analysis (Griffiths et al., 2007b). Similarly, there was no effect of purified Cry1Ab, Cry3A, or Cry4 protein on growth of selected bacteria ( 8 gram-negative, 5 gram-positive, and a cyanobacterium) in vitro, in pure and mixed cultures, using dilution, disk-diffusion, and sporulation assays (Koskella and Stotzky, 2002).

In studies where biomass of $B t$ plants was added to soil, there were generally no negative effects on the numbers of culturable bacteria (Saxena and Stotzky, 2001a; Flores et al., 2005) or on soil microbial community structure as determined by DGGE (Tan et al., 2010). In decomposition studies, there was no difference in bacterial growth on $B t$ maize versus non-Bt maize leaves, however, bacterial growth was lower on the feces of wood lice (Porcellio scaber) that were fed Bt maize leaves (Escher et al., 2000). There were no negative effects on bacterial decomposer communities of litterbags containing $B t$ maize biomass (cobs, roots, or stems plus leaves) (Xue et al., 2011) or Bt rice biomass (straw or roots) (Lu et al., 2010a; Lu et al., 2010b) as demonstrated though terminal restriction fragment length polymorphism (T-RFLP) analysis, nor was there a direct toxic effect on culturable bacteria in soil amended with $B t$ rice straw (Wu et al., 2004b). When ground, dried roots and shoots of $B t$ cotton or non- $B t$ cotton were mixed with fieldcollected soil and incubated for two months, there was no significant difference in urease, phosphatase, dehydrogenase, phenol oxidase, or protease activities between the $B t$ and non- $B t$ biomass treatments, however, enzyme activity was stimulated by the addition of 
both $B t$ and non-Bt cotton biomass (Shen et al., 2006).

In soil cultivated with $B t$ or non- $B t$ maize in the laboratory, Saxena and Stotzky (2001a) detected no significant difference in the CFUs of culturable bacteria (including actinomycetes) between rhizosphere soil of $B t$ and non- $B t$ maize after 45 days or between soil amended with $B t$ and non-Bt maize biomass (Saxena and Stotzky, 2001a). When $B t$ maize and non- $B t$ maize were cultivated in a growth chamber, there was no difference in bacterial community structure detected between bulk soil of $B t$ and non-Bt maize using PLFA analysis (Blackwood and Buyer, 2004). Although a small difference in rhizosphere bacterial community structure was detected in the $B t$ versus non- $B t$ maize cultivated soil (as determined by community-level physiological profiles [CLPP]), the differences in the rhizosphere microbial community between the $B t$ and non- $B t$ maize cultivated soil were most influenced by soil type and texture (Blackwood and Buyer, 2004).

In greenhouse studies, there was also no difference in rhizosphere bacterial community structure in soil cultivated with $B t$ maize and non- $B t$ maize as determined by community level catabolic profiling (CLCP) (Brusetti et al., 2004). However, when soil was treated with a root exudate solution collected from $B t$ or non- $B t$ maize plants grown hydroponically, differences in bacterial community structure were detected by automated ribosomal intergenic spacer analysis (ARISA), suggesting that changes in root exudates may influence the rhizosphere bacterial community more than the expression of $B t$ protein (Brusetti et al., 2004). Using DGGE analysis and 16S rRNA gene sequences, Tan 
et al. (2010) detected no difference in microbial community structure between soils cultivated with two different lines of $B t$ maize and corresponding non-Bt maize lines. Similarly, there was no difference in microbial community structure as determined by PLFA between $B t$ maize and non- $B t$ maize grown in greenhouse pots using soil collected from field plots that had been cultivated with the same $B t$ and non-Bt cultivars (Griffiths et al., 2006). When the effects of eight different Bt maize lines (expressing CrylAb) and their corresponding non- $B t$ maize isolines on soil microbes were evaluated using PLFA analysis, Griffiths et al. (2007b) reported that although soil microbial community structure was significantly affected by the growth stage of the plant, it was not affected by the $B t$ gene insertion. There were also no negative effects of the cultivation of $B t$ cotton on species richness or functional diversity of rhizosphere microbial communities as determined by Biolog assays or on enzyme activities (urease, phosphatase, dehydrogenase, phenol oxidase, and protease) when compared to soil cultivated with non-Bt cotton (Shen et al., 2006).

Most field studies have also demonstrated no significant negative effects of $B t$ crop cultivation on soil microbes. In a two year field experiment, there were no effects of $B t$ maize cultivation on microbial activity ( $\mathrm{N}$ mineralization potential, short-term nitrification rate, and soil respiration) or bacterial community structure detected by $\mathrm{T}$ RFLP analysis when compared to soil cultivated with a non- $B t$ maize isoline (Devare et al., 2004). After three years, there were still no significant differences in microbial 
biomass or microbial activity detected in the $B t$ versus non- $B t$ maize plots, although there were seasonal variations in microbial biomass and activity (Devare et al., 2007). In rhizosphere soils collected from $B t$ maize and three different non- $B t$ maize cultivars over three years, there were no differences in rhizosphere bacterial community structure as determined by polymerase chain reaction (PCR) amplification of 16S rRNA genes (Miethling-Graff et al., 2010), nor was there a difference in bacterial community structure between rhizosphere soils of $B t$ maize and two non- $B t$ maize lines grown for two years at three different European field sites (Denmark, Eastern France, South-West France) as determined through PLFA analysis and CLPP (Griffiths et al., 2005). There were also no differences in the culturable aerobic bacteria (including actinomycetes), dehydrogenase and nitrogenase activities, or ATP content, in rhizosphere soils of $B t$ maize versus non- $B t$ maize over a two year field study, although there were seasonal variations as well as significant differences between rhizosphere and bulk soil samples (Oliveira et al., 2008). When soils were cultivated for four years with four different lines of $B t$ maize (two lines of Bt 11; Cry1 Ab [sweet corn and field corn], Event MON810; Cry1Ab, and Event MON863; Cry3Bb1) and non-Bt maize, there was no difference in microbial diversity detected through dilution plating and DGGE or in enzyme activities (arylsulfatases, acid and alkaline phosphatases, dehydrogenases, and proteases) (Icoz et al., 2008). Using metabolic profiling and molecular analysis of 16S rRNA genes, Fang et al. (2005) determined that rhizosphere bacterial diversity was affected more by soil texture than by 
cultivation with $B t$ maize in both greenhouse and field studies. Similarly, in soil cultivated with $B t$ maize and non-Bt maize, the rhizosphere bacterial community structure was more affected by environmental factors such as the ontogeny of the plants or heterogeneities within the field soil than by the Cry1Ab protein expressed in the $B t$ maize plants as determined by SSCP (single-strand conformation polymorphism) of PCRamplified 16S rRNA genes (Baumgarte and Tebbe 2005). When soil was cultivated with $B t$ cotton, Hu et al. (2009) found no negative effects of the $B t$ cultivar on rhizosphere bacteria, and Li et al. (2011) reported no differences in Azotobacter, denitrifying bacteria, ammonia-oxidizing bacteria, or microbial diversity between soil cultivated with $B t$ and non- $B t$ cotton over a three year field study as determined by selective plating and most probable number (MPN) assays, although there were seasonal variations not related to the $B t$ or non-Bt cultivars. When soil enzyme activities (phosphatase activity, dehydrogenase activity, respiration, and methanogenesis) and microbial community composition were compared in the rhizosphere of $B t$ rice, non- $B t$ parental rice, and non- $B t$ parental rice treated with the insecticide Triazophos at multiple sampling times, there were generally no significant negative effects detected on soil enzyme activity or microbial community structure as determined by DGGE and T-RFLP (Liu et al., 2008). There were, however, seasonal variations in the selected enzyme activities and microbial community composition in the rhizosphere over the course of the two year experiment (Liu et al., 2008). There was no negative effect of four years of cultivation with $B t$ spruce 
(engineered to express Cry1 $\mathrm{Ab}$ ) on nitrogen-fixing bacteria compared with non- $B t$ white spruce trees as determined by molecular sequencing of a region of the nitrogenase reductase gene from genomic DNA extracted from rhizosphere soil (Lamarche and Hamelin, 2007). There were also minimal differences in culturable aerobic bacteria in rhizosphere soil cultivated with $B t$ potato, non- $B t$ Russet potato treated with insecticide (Di-Syston), and non-Bt Russet potato treated with microbial Bt (M-Trak) (Donegan et al., 1996). When the microflora colonizing the leaves of these potato plants were compared over multiple time points (0, 21, 42, 63, and 98 days), Donegan et al. (1996) found few significant differences across potato cultivars.

These, and other, results indicate that, in general, the insecticidal Bt proteins, either purified or expressed in transgenic Bt plants, have no significant negative effects on most soil bacteria. However, the few studies where effects of cultivation of $B t$ plants on soil microbes were observed (e.g., Donegan et al., 1995; Wu et al., 2004a; Wu et al., 2004b; Castaldini et al., 2005; Rui et al., 2005; Xue et al., 2005; Fang et al., 2007; Sun et al., 2007; Chen et al., 2011), differences in physiological properties within plants resulting from the genetic insertion may be implicated (e.g., Donegan et al., 1995; Rui et al., 2005). Genetic alterations, as a result of the insertion of $B t$ genes, that produce a change in plant root exudates and/or quality of plant material, for example, may influence microbial growth and species composition in the rhizosphere and/or affect the degradation time or quality of $B t$ plant litter. In this way, microbial communities could be 
affected by the cultivation of transgenic $B t$ crops without being negatively affected by $B t$ proteins directly. Fluxes in microbial community structure, however, can also be influenced by soil type, temperature, season, plant type, and other biotic and abiotic factors (e.g., Griffiths, 2000; Lottman, 2000; Kowalchuk, 2002; Dunfield and Germida, 2003; Zwahlen et al., 2003a; Blackwood and Buyer, 2004; Icoz and Stotzky, 2008b). Thus, where an impact of the cultivation of a $B t$ crop on soil bacteria has been detected, the ecological significance has often been difficult to assess.

Research recommendations: Effects of the Cultivation of $B t$ Crops on Soil Bacteria Most of the studies evaluating nontarget effects of $B t$ crops or $B t$ proteins on soil bacteria have examined effects on culturable bacteria (Table 3). Given the fact that less than $1 \%$ of bacterial taxa are thought to be culturable (e.g., Handelsman and Tiedje, 2007), this methodology could influence the results of many of these studies. Thus, differences in laboratory techniques may also have a role in the different outcomes of similar studies evaluating the effects of $B t$ crops on microbial communities (e.g., plating vs. DGGE vs. metabolic analysis). To evaluate the nontarget effects of the cultivation of $B t$ crops on soil bacteria, multiple detection methods should be employed, as most microbes are not culturable and could be better identified, quantified, or characterized using a combination of molecular and metabolic tools. Risk assessment studies should also be conducted at multiple levels (laboratory, greenhouse, and field), and under 
different experimental and environmental conditions, as it is well known that bacteria and other soil organisms can be easily influenced by a multitude of biotic and abiotic factors that may not be associated with GM plants. As relatively few significant negative impacts of $B t$ crops have been reported for soil bacteria, it may be more useful to focus future research efforts on other soil organisms that are more closely associated with roots (e.g., mycorrhizal fungi) or narrowing the scope of bacterial research to investigate the effects of transgenic crops on nitrogen-fixing bacteria, many of which can form symbiotic relationships with plant roots and have clear benefits for plant health and ecosystem function by providing nutrients to plants and protection against plant pathogens (e.g., reviewed in Sessitsch et al., 2002; Dobbelaere et al., 2003; Hayat et al., 2010).

\section{Effects of the cultivation of Bt crops on soil fungi}

Soil fungi, including saprophytic, parasitic/pathogenic, and mycorrhizal fungi, are another group of soil organisms that may be affected by cultivation of transgenic Bt crops (Table 4). Fungi have important roles in the soil ecosystem as decomposers, nutrient recyclers, plant symbionts, and plant pathogens. Saprotrophic fungi (also called saprophytic fungi) are the primary group of soil organisms that degrade organic material in agricultural fields, whereas parasitic/pathogenic fungi and mycorrhizal fungi are found in close association with living plant roots in the rhizosphere.

Saprotrophic fungi are free-living soil organisms that obtain their nutrients from dead 
organic material, such as leaves, wood, and other plant and animal materials, and are responsible for recycling a significant amount of the carbon in the soil ecosystem. To date, most studies have determined that there is no significant negative effect of purified $B t$ proteins, $B t$ crop cultivation, or $B t$ plant material on saprotrophic fungi and/or culturable fungi (Table 4) (e.g., Saxena and Stotzky, 2001a; Koskella and Stotzky, 2002; Ferreira et al., 2003; Icoz et al., 2008; Oliveira et al., 2008). For example, when soil was amended with purified CrylAb or CrylAc protein, there was no difference in the population levels of culturable fungi using selective plating compared with control soil (Donegan et al., 1995). There was also no effect of the addition of purified Bt proteins (Cry1Ab, Cry3A, or Cry4) on the growth of representative culturable fungi (Cunninghamela elegans, Rhizopus nigricans, Aspergillus niger, Fusarium solani, Penicillium sp., Saccharomyces cerevisiae, and Candida albicans) in pure and mixed cultures (Koskella and Stotzky, 2002).

Where significant effects have been reported, they have often been minor or transient. When soybean was grown in soil inoculated with a strain of Bacillus thuringiensis bacteria that expressed CrylAb protein (Cry+), a Bt mutant strain that did not express Cry protein (Cry-), purified insecticidal crystal protein (ICP), or no treatment (control), there was no significant difference in the culturable fungal populations between rhizosphere soils, however, there was a transient increase in some functional groups (saprophytic, amylolytic, cellulolytic, and proteolytic fungi) between the treatments as 
determined by selective plating, at the beginning of the experiment (Ferreira et al., 2003). Donegan et al. (1995) also reported a transient increase in culturable fungi in soil amended with $B t$ cotton based on selective plating methods. However, Flores et al. (2005) and Saxena and Stotzky (2001a) found no difference in culturable fungi between soils amended with $B t$ and non-Bt maize in soil microcosms. There were also no negative effects on the numbers of culturable fungi in flooded soils amended with $B t$ versus non- $B t$ rice straw in laboratory experiments (Wu et al., 2004b). In litterbag decomposition field studies, there was no difference in fungal community composition between $B t$ and non- $B t$ rice straw samples as determined by T-RFLP, although there were some differences in fungal community composition at the early stage of rice root decomposition ( $\mathrm{Lu}$ et al., 2010a). In a greenhouse study, Tan et al. (2010) reported that neither actively growing $B t$ maize nor the incorporation of $B t$ maize biomass (leaves and straw) had a negative effect on fungal community structure in soil as determined by PCR-DGGE and sequences of 18S rRNA genes. Although, Xue et al. (2011) found a minor effect (1 out of 16 comparisons) of $B t$ maize biomass buried in litterbags on fungal decomposer communities as determined by T-RFLP, the differences were mostly due to environmental factors (i.e., litterbag placement, recovery year, and plot history) and were not a result of $\mathrm{Cry} 3 \mathrm{Bb}$ protein in the $B t$ maize.

In growth chamber experiments, Saxena and Stotzky (2001a) found no difference in the numbers of selected culturable Zygomycetes, Ascomycetes, Deuteromycetes, and 
yeasts in rhizosphere soils cultivated with $B t$ and non- $B t$ maize. However, Blackwood and Buyer (2004) reported that soils cultivated with $B t$ maize expressing Cry $1 \mathrm{Ab}$ and Cry $1 \mathrm{~F}$ reduced the presence of eukaryotic PLFA in bulk soils compared with soils cultivated with non- $B t$ maize, although it was not clear which groups of eukaryotes were affected. In field soils cultivated with $B t$ and non- $B t$ maize, Icoz et al. (2008) and Oliveira et al. (2008) found no consistent effect of $B t$ maize cultivation on culturable fungi. Similarly, there was no difference in fungal to bacterial ratio reported between field soils cultivated with $B t$ maize and non- $B t$ maize as determined by PLFA and culturing methods (Xue et al., 2005). Li et al. (2011) reported a seasonal variation in numbers of CFUs of culturable fungi in soils cultivated with $B t$ and non-Bt cotton over a three-year field study, but there was no negative effect of $B t$ cotton cultivation on soil fungi.

Although it is not surprising that Bt proteins in transgenic plant material have little or no direct effect on saprotrophic fungi, it was hypothesized that the higher lignin content reported in the biomass of some $B t$ cultivars (Saxena and Stotzky, 2001b; Stotzky, 2004; Flores et al., 2005; Poerschmann et al., 2005) might take longer for fungi to degrade, thus leading to accumulation of $B t$ plant residue in the soil over time. This has turned out not to be true most of the time. Although one study showed that some Bt plants, including maize, canola, potato, rice, and tobacco, decomposed less in soil and linked this effect to the higher (although not always significantly higher) lignin content in each of the $B t$ cultivars tested (Flores et al., 2005), several subsequent studies have reported that $B t$ 
plant residue does not generally decompose more slowly than non-Bt plant material (Lehman et al., 2008; Tarkalson et al., 2008; Kravchenko et al., 2009; Wu et al., 2009). Moreover, some studies have not even been able to detect a difference in lignin content between $B t$ and non-Bt cultivars (Jung and Sheaffer, 2004; Mungai et al., 2005; Lang et al., 2006). These contrasting reports may be the result of differences in age of the plants, detection techniques, or cultivar, highlighting the importance of a plant line-specific, multi-detection, multiple sampling time approach to determine more accurately the effects of the composition of $B t$ crop material on organisms in the soil ecosystem.

Parasitic and pathogenic fungi are also prevalent in soil, and although not desirable in agricultural systems, they may also be affected by changes in the physiology of crop plants. However, during the plant selection process when GM plants are designed and tested, it is unlikely that genotypes that are more susceptible to disease would be released for commercial application. One study that examined the effects of $B t$ crops on fungal pathogens found that genetically engineered $B t$ potato had no negative effect on soilborne pathogens in the rhizosphere, including Fusarium sp., Pythium sp., Verticillium dahliae, potato leaf roll virus, and potato virus Y, under field conditions (Donegan et al., 1996). When fungal growth and survival of the plant pathogen, Fusarium graminearum, and its antagonist, Trichoderma atroviride, were evaluated in flask experiments using pulverized leaf tissue from four different $B t$ maize hybrids (and their corresponding non$B t$ isolines), both fungal species degraded the Cryl $\mathrm{Ab}$ protein in the $B t$ maize tissue and 
there was no consistent difference in fungal growth on leaf tissue from $B t$ versus non- $B t$ maize plants as determined by PCR quantification of fungal biomass (Naef et al., 2006). There was also no effect of purified Cry1 Ab protein on fungal growth in agar plate assays (Naef et al., 2006). Interestingly, some of the paired $B t$ and non- $B t$ maize lines differed more in volatile organic compound composition than could be accounted for by just the presence of the Cry protein alone (Naef et al., 2006). This study provides an example of a pleiotropic effect that can occur in GM plants that could alter the quality or composition of transgenic plant tissue, potentially affecting degradation time and/or the structure of fungal communities inhabiting leaf material.

Of all the different types of fungi in the soil ecosystem, arbuscular mycorrhizal fungi (AMF) may be at the most risk for nontarget effects of transgenic $B t$ crop cultivation because of their close association with plant roots and their obligately biotrophic nature. Mycorrhizal fungi form symbiotic relationships with $92 \%$ of terrestrial plant families (Wang and Qiu, 2006) and are generally considered to be beneficial to plant growth and ecosystem health (Smith and Read, 2008). The mycorrhizal symbiosis is a result of bidirectional exchange: plants supply sugars to the mycorrhizal fungi, and the fungi benefit plants by improving nutrient and water acquisition (Smith and Read, 2008). Of particular importance to plant health is the ability of AMF to obtain essential minerals such as phosphorus and zinc, which are often found in soil in forms that are unavailable to plants (Vance et al., 2003; Rillig, 2004; Morgan et al., 2005; Smith and Read, 2008). 
AMF are also important for nutrient acquisition in low nutrient environments and in the absence of synthetic chemical fertilizers (Gosling et al., 2006; Lekberg et al., 2008; Sheng et al., 2008). Because AMF are obligate symbionts that require a plant host for nutrition and reproduction, they may be uniquely sensitive to alterations in plant physiology as a result of a genetic insertion, Bt protein accumulation in the root zone, and/or to alterations in root exudates or plant tissue composition.

Although AMF have an important role in maintaining plant and ecosystem health, few environmental risk assessment studies have examined nontarget effects of Bt plants on AMF (Table 4; reviewed by Icoz and Stotzky, 2008b; Liu and Du, 2008; Liu, 2010). In one of the first studies that evaluated the impact of $B t$ crop plants on AMF, Turrini et al. (2004) found that root exudates of Bt maize (Event Bt 176) significantly reduced presymbiotic hyphal growth of the AMF species, Glomus mosseae, and that $36 \%$ of the appressoria (swollen, flattened fungal filaments that enable fungal invasion) failed to produce viable infection pegs in the roots of the $B t$ maize cultivar. The fungal symbionts were able to locate their host, but they were unable to establish the same amount of colonization as in the non- $B t$ isoline, suggesting that the host recognition mechanisms of the fungus were not disrupted but that something at the plant/fungal interface may have limited colonization. In soil microcosms, $B t$ maize (Event $B t$ 176) again exhibited a lower level of early mycorrhizal colonization and arbuscule development when compared with another line of $B t$ maize (Event $B t 11)$ and a non- $B t$ parental isoline, although both $B t$ 
cultivars had less arbuscule formation than the parental maize line (Castaldini et al., 2005). In a greenhouse study, the total infected root length of the Bt maize plants (Events Bt 11 and Bt 176) was $50 \%$ less than that of the parental maize line (Castaldini et al., 2005). Conversely, de Vaufleury et al. (2007) found no difference in frequency or intensity of root colonization by AMF between $B t$ maize (Event MON810) and its non- $B t$ isoline (Monumental) in soil microcosms, despite the fact that Event MON810 expresses the same type of Cry1 Ab protein as Events $B t 11$ and $B t$ 176. In field studies, Knox et al. (2008) also found no difference in colonization by AMF arbuscules between $B t$ cotton (Cry1 Ac, Cry2Ab) and non-Bt cotton. As each of these studies differed in experimental conditions (soil type, plant genotype, species of AMF, fertilizer level, spore number, location of study - microcosm, greenhouse, or field, AMF detection technique, etc.), it has been difficult to identify the primary factors influencing the patterns of AMF colonization in the different $B t$ cultivars.

When the environmental and experimental factors that may influence the symbiotic relationship between $B t$ maize and AMF were evaluated in a greenhouse study, Cheeke et al. (2011) found that significant differences in AMF colonization were only observed between $B t$ maize (Event $B t$ 11) and its non- $B t$ isoline (Providence) when fertilizer levels were limited and AMF spore density was high (80 spores of G. mosseae/pot). Under these experimental conditions, $B t$ maize roots had less than $50 \%$ of the AMF colonization of the non-Bt isoline (Figure 1.1). This study demonstrated that under circumstances 
where AMF would be most likely to colonize and benefit the host plant (high spore density, low fertilizer conditions) mycorrhizal colonization was limited in the $B t$ maize. This study also showed that plant response to AMF is dynamic and AMF colonization levels by $G$. mosseae can vary, even within the same $B t$ or non- $B t$ maize cultivar, depending on the level of fertilizer application or number of spores added to soil (Figure 1) (Cheeke et al., 2011). In a follow up greenhouse study where AMF colonization levels by fungi from field collected soil were evaluated in 9 paired lines of $B t$ and non- $B t$ maize, $B t$ maize plants overall had lower levels of AMF colonization in roots than the non-Bt maize plants, when fertilizer was limited (Cheeke et al., 2012).

The effect of $B t$ plant material incorporated into soil on AMF has also been investigated. In a greenhouse study, Castaldini et al. (2005) observed that four months after $B t$ maize biomass (Event $B t 11$ ) was incorporated into soil, the percent of root colonization by AMF in Medicago sativa (alfalfa) was significantly lower in soil containing $B t$ maize residue than in $M$. sativa plants grown in soil amended with non- $B t$ maize biomass. Although no mechanism for these results was identified, a change in nutrient composition of $B t$ plant biomass, lignin content, and/or time required for degradation of transgenic plant material could potentially contribute to indirect effects on $\mathrm{AMF}$ in the soil. Interestingly, when soybean was grown in soil inoculated with a strain of Bacillus thuringiensis that expressed Cry1Ab protein (Cry+), a Bt mutant bacterial strain that did not express Cry protein (Cry-), purified insecticidal crystal protein (ICP), 
or no treatment (control), Ferreira et al. (2003) found that there was no effect of the addition of purified ICP on AMF, but that Cry+ and Cry- bacterial strains inhibited AMF compared to the control treatment.

Research recommendations: Effects of the cultivation of $B t$ crops on soil fungi In general, there appear to be no negative effects of $B t$ plants, $B t$ proteins, or the cultivation of $B t$ crops on most free-living fungal species, including saprophytic and pathogenic fungi. However, AMF seem to be sensitive to some Bt plant lines, perhaps because of their symbiotic relationship with host plants. As the effect does not appear to be protein-specific, each $B t$ plant line containing a different insertion event should be screened for its ability to form mycorrhizal relationships. Currently, there are at least 15 different lines of $B t$ maize genetically engineered to include single, stacked, or pyramided resistance traits, yet few of these $B t$ cultivars have been evaluated for their ability to form symbioses with AMF. Because of the initial indications that certain cultivars of $B t$ maize are poorly colonized by AMF (Turrini et al., 2004; Castaldini et al., 2005; Cheeke et al., 2011, Cheeke et al., 2012), and that each Bt line may have a different response to mycorrhizal fungi, it is important to test the ability of each $B t$ line to form associations with AMF under the same experimental conditions, as it may not be possible to generalize about the effects of $B t$ maize on AMF, especially if the effect is not proteinspecific. As most agriculturally important crops are mycorrhizal (excluding species of 
Brassica), even small impacts of $B t$ crops on their fungal symbionts should be closely monitored, particularly in low-input farming systems where reliance on a healthy soil community for plant health and nutrition is key. More research, including screening of multiple $B t$ cultivars for AMF responsiveness and molecular identification of the taxa of AMF colonizing $B t$ and non- $B t$ plant roots should be conducted, to determine whether $B t$ plants with lower levels of AMF colonization also have reduced diversity of AMF in their roots. If this is the case, it would then be pertinent to evaluate changes in spore abundance and diversity of AMF over time in soils in regions where $B t$ crops have been cultivated for multiple years.

\section{Effects of the cultivation of Bt crops on protozoa and nematodes}

Protozoa (amoebae, flagellates, and ciliates) and nematodes have an important role in the soil ecosystem, primarily as grazers. By consuming bacteria and other soil organisms, they release nutrients into the soil that can be used as a food source by other soil biota or taken up by plant roots. Protozoa are unicellular organisms that are motile in both aquatic and soil environments and feed on bacteria, small fungi, algae, and other protozoa. Nematodes are microscopic worms that are classified into several trophic groups bacterial feeders, fungal feeders, and root feeders, primarily based their mouth parts (e.g., whether they have a sharp stylet for piercing root or fungal tissue). Because of their importance in nutrient turnover in soil food webs and their close proximity to plant roots 
(reviewed by Bais et al., 2006), protozoa and nematodes are another group of soil organisms that may be impacted by cultivation of transgenic $B t$ crops.

Few studies have evaluated the impact of $B t$ plants on protozoa, and the results to date are inconsistent (Table 1.5). Some studies have reported no effect on protozoa in soil amended with purified Cry1 Ab or Cry1Ac protein (Donegan et al., 1995) or with $B t$ maize biomass expressing Cry1Ab (Saxena and Stotzky 2001a), whereas others have reported both higher (Griffiths et al., 2006; Griffiths et al., 2007a) and lower numbers of protozoa (Griffiths et al., 2005) in soil cultivated with Bt maize (expressing Cry1Ab) in the greenhouse and in the field (reviewed in Birch et al., 2007). There was no significant difference in protozoa numbers between soil cultivated with $B t$ maize (Cry1 Ab) and non$B t$ maize in a plant growth room (Saxena and Stotzky, 2001a) or in soil cultivated with $B t$ (Cry1Ab and Cry3Bb1) and non-Bt maize in the field (Icoz et al., 2008). This variation in results, even in studies conducted by the same researchers, could be the result of differences in experimental or environmental factors, such as sampling time or different ecological conditions in the greenhouse and the field (e.g., Griffiths et al., 2007a). Moreover, because amoebae and flagellates tend to be more sensitive to their environmental conditions (e.g., soil compaction, tillage, anoxic conditions) than ciliates, for example, it is important to evaluate each class of protozoa separately, as they might each have a different response to changes in agricultural practices resulting from the cultivation of transgenic crops and/or differences in $B t$ root exudates or Bt plant biomass. 
Research on the nontarget impact of $B t$ crops on nematodes indicates that negative effects on this group of soil organisms may indeed be Bt protein specific (Table 1.6). Some Bt proteins (Cry5, Cry6, Cry12, Cry13, Cry14, Cry21) have been shown to have a direct toxicity to nematodes (e.g., Meadows et al., 1989a; Meadows et al., 1989b; Meadows et al., 1990; Marroquin et al., 2000; Kotze et al., 2005; Hu et al., 2010; Hoess et al., 2011), and some are used as biological control agents (i.e., Cry5, Cry6) to protect plants from root-feeding nematodes (reviewed in Bravo et al., 2007; Li et al., 2007a; Li et al., 2007b; Li et al., 2008; Khan et al., 2010). Caenorhabditis elegans, a common model nematode that is found in a variety of natural and agroecosystems, appears to be negatively impacted by some $B t$ proteins, including Cry1 Ab, Cry3Bb1, Cry5A, and Cry5B. In field studies, a lower abundance of C. elegans was detected in soils cultivated with $B t$ maize expressing Cry $1 \mathrm{Ab}$ than in soils with non- $B t$ maize (Manachini and Lozzia, 2003), and there was a negative effect of the Cry1 Ab protein from Bt maize on nematode growth, number of eggs, and reproduction of C. elegans (Lang et al., 2006; Hoess et al., 2008). When C. elegans was fed purified Cry5A and Cry5B proteins, individuals showed substantial gut damage, decreased fertility, and increased mortality, consistent with what would be expected in target insect populations (Marroquin et al., 2000). Hoess et al. (2011) found a dose-dependent negative response on the growth and reproduction of $C$. elegans to purified $\mathrm{Cry} 3 \mathrm{Bb} 1$ using a bioassay, and gene expression analysis demonstrated that Cry-protein specific defense genes were up-regulated in the 
presence of Cry1 Ab or Cry3Bb1 protein. However, in a field studies with Bt maize that expressed a different protein - Cry3Bb1, there was no negative effect on C. elegans (AlDeeb et al., 2003; Hoess et al., 2011), probably because of the low level of Cry3Bb1 protein in the rhizosphere (Hoess et al., 2011). Nematode abundance and diversity were also not different in the rhizospheres of $B t$ and non- $B t$ maize, however, a shift in nematode genus composition occurred in two of the three the $B t$ maize plots at the end of the field season, but it did not affect functional diversity (Hoess et al., 2011)

The toxic effects of CrylAb observed in C. elegans have not been detected in other nematode species but, in some cases, nematode communities have been affected by the cultivation of $B t$ maize. Greenhouse experiments demonstrated that populations of Acrobeloides sp. and Pratylenchus sp. were significantly higher in soil under cultivation with $B t$ maize (Cry1 Ab) than with non-Bt maize (Griffiths et al., 2006). However, in field trials, Lang et al. (2006) found no effect of Bt maize (Cry1 Ab) cultivation on Pratylenchus sp. When natural nematode communities were evaluated in the field, cultivation of $B t$ maize expressing Cry1Ab significantly reduced numbers of nematodes in the soil, although the effect was small and within the normal variation observed in many agricultural systems (Griffiths et al., 2005). Cultivation of $B t$ canola expressing CrylAc was associated with a shift in nematode community structure when compared with community structure in soil cultivated with non-Bt canola (Manachini et al., 2004). Other studies have shown that Bt maize expressing CrylAb has no negative effect on 
natural populations of nematodes in soil microcosms (Saxena and Stotzky, 2001a) or in the field (Manachini and Lozzia, 2002) and that Bt eggplant expressing Cry3Bb1 has no negative effect on nematode community structure in the field (Manachini et al., 2003).

Research recommendations: Effects of the cultivation of $B t$ crops on protozoa and nematodes

Because few studies have evaluated the effects of the cultivation of $B t$ crops on protozoa, the results reported to date are difficult to assess. However, there appear to be no consistent negative effects of purified Bt proteins (Cry1Ab and Cry1Ac) or the cultivation of $B t$ maize (expressing Cry1 Ab or Cry3Bb1) on protozoa in greenhouse or in field studies. Future studies would benefit from evaluating a greater variety of $B t$ crops for nontarget effects on protozoa in the soil, and under a range of experimental conditions. Moreover, data should be reported separately for the different classes of protozoa (amoebae, flagellates, and ciliates), as each group may have a different response to $B t$ proteins, $B t$ residue in the soil, and/or the cultivation of $B t$ crops.

Although nematode community structure, biodiversity, and number of individuals in natural populations do not appear to be affected by cultivation of $B t$ crops, individual species, such as C. elegans, appear to be sensitive to some Cry proteins, including the $\mathrm{Cry} 1 \mathrm{Ab}$ protein that is expressed in most lines of transgenic maize. As $B t$ maize is one of the most commonly cultivated transgenic crops worldwide, and as nematodes are key 
indicators of soil quality (Blair et al., 1996), more research on the impacts of Cry1Ab and other $B t$ proteins should be conducted to evaluate nontarget effects both at the individual and population level of nematodes. Insomuch as nematodes are one of the only groups of soil organisms that have displayed direct toxicity of certain Cry proteins produced in transgenic $B t$ crops, they should be carefully evaluated for nontarget effects of $B t$ crop cultivation under a variety of environmental and experimental scenarios.

Effects of the cultivation of Bt crops on earthworms

Earthworms are responsible for much of the initial degradation of plant material, incorporation of organic matter into lower strata of soil, and increase in aeration of soil by creating large pores in soil as they burrow. Impacts of $B t$ plant biomass, $B t$ proteins, and the cultivation of $B t$ crops on a variety of earthworm species have been studied in numerous laboratory, greenhouse, and field experiments, and most studies have shown that there are few or no effects on this group of soil organisms (Table 1.7; reviewed by: Stotzky, 2004; O'Callaghan et al., 2005; Birch et al., 2007; Icoz and Stotzky, 2008b). In laboratory studies, there was no negative effect of soil amended with biomass of Bt maize expressing Cry1Ab on Eisenia fetida mortality and weight (Ahl Goy et al., 1995), nor were there deleterious effects on survival and reproduction of $E$. fetida fed leaves of $B t$ maize expressing Cry1Ab (Clark and Coats, 2006). Similarly, Saxena and Stotzky (2001a) found no significant difference in mortality and weight of Lumbricus terrestris 
grown for 40 days in soil cultivated with $B t$ maize (Cry1 Ab) or non-Bt maize, and no effect on mortality and weight of $L$. terrestris grown in soil amended with ground, dried $B t$ maize biomass for 45 days. No lethal effects of $B t$ maize (Cry1Ab) residues on adult or immature earthworms were found in field studies (Zwahlen et al., 2003b; Lang et al., 2006; Zwahlen et al., 2007). However, adult L. terrestris had a significant loss in weight in a laboratory experiment when worms were grown in soil amended with $B t(\mathrm{Cry} 1 \mathrm{Ab})$ versus non-Bt maize biomass in glass tubes for 200 days (Zwahlen et al., 2003b). Laboratory studies with Aporrectodea caliginosa reported no negative effect on survival, growth, development, or reproduction of individuals grown in soil amended with leaves of $B t$ maize (Cry1Ab) or grown in pots cultivated with $B t$ maize (Vercesi et al., 2006), and there was no effect on survival and reproduction of Enchytraeus albidus that were fed diets with $B t$ maize (Cry3Bb1) versus non- $B t$ maize leaf biomass (Honemann and Nentwig, 2009). However, E. albidus fed Bt leaves (Cry1Ab) had a lower reproduction rate but a higher survival rate when compared with worms fed leaves of the non-Bt maize isoline (Honemann and Nentwig, 2009). When litterbags containing biomass of nine different $B t$ maize (Cry1 $\mathrm{Ab}$ and $\mathrm{Cry} 3 \mathrm{Bb} 1)$ and non- $B t$ maize cultivars were buried in the field, there was no difference in the numbers of Enchytraeidae or Lumbricidae found in litterbags after nine months, nor was there a difference in the degradation rate between the different maize varieties (Honemann et al., 2008). Interestingly, when maize residues were added to soil microcosms, consumption of Bt plant material by L. terrestris and $A$. 
caliginosa lowered the concentration of immunoreactive Cry1 Ab protein in soil to less than $10 \%$ of the original concentration after 5 weeks, without causing any detectable harm on the earthworms (Schrader et al., 2008). E. fetida fed Bt cotton leaves expressing CrylAc in the laboratory also displayed no toxic effects, and even resulted in slight increases in growth and reproduction compared with earthworms fed non-Bt cotton leaves, although this difference was not statistically significant (Liu et al., 2009a; Liu et al., 2009b).

In other studies, there have been no negative effects reported of the cultivation of $B t$ maize expressing Cry3Bb1 on the abundance of Oligocheata in the field (Bhatti et al., $2005)$ or soil planted with or amended with $B t$ maize (Cry3Bb1) on weight and mortality of L. terrestris in the greenhouse (Ahmad et al., 2006). There was also no difference in the biomass of juveniles or adults of A. caliginosa, Aporrectodea trapezoides, Aporrectodea tuberculata and L. terrestris in soil cultivated with Bt maize expressing Cry1 Ab and Cry3Bb1 compared to soil cultivated with non-Bt maize over a four-year field study (Zeilinger et al., 2010). When effects of the cultivation of Bt maize (CrylAb) and herbicide-tolerant (HT) maize were tested on natural populations of earthworms (including A. caliginosa, Aporrectodea longa, Aporrectodea rosea, L. terrestris, Allolobophora chlorotica, Prosellodrilus amplisetosus, and Allolobophora cupulifera) over two consecutive growing seasons at two different field sites, earthworm populations were reduced only in the plots with the HT crop, likely due to reduced tillage (RT) 
practices associated with HT crops (Krogh et al., 2007). Plots with the HT maize allowed the practice of reduced tillage; plants can be sprayed with herbicides for weed control rather than using tillage measures. In the HT, RT plots, earthworm populations were significantly reduced to about half of earthworm populations in the HT plots that received the conventional tillage treatment. The authors speculated that the reduction in earthworm populations in the HT plots was likely because of exposure to the herbicide Basta $\AA$, as the HT maize plants were the same across treatments (Krogh et al., 2007).

Research recommendations: Effects of the cultivation of $B t$ crops on earthworms The laboratory, greenhouse, and field studies outlined above indicate that cultivation of $B t$ crops, including maize expressing Cry1 Ab or Cry3Bb1 and cotton expressing Cry1Ac, has no deleterious effects in soil on numbers or populations of earthworms, and that differences in agricultural practices (e.g. herbicide applications) associated with the cultivation of GM crops may be more important to earthworm health and ecosystem function than the $B t$ proteins associated with GM crops. Nevertheless, continued monitoring for effects of $B t$ plants on earthworms would be useful, especially with the ever-increasing hectarage dedicated to the cultivation of $B t$ crops across the globe and the continued development of new and different types of $B t$ plants. Because earthworms do not appear to be affected by most $B t$ plants examined thus far, in future studies, the research focus should be narrowed to evaluate earthworm species based on their 
association with a particular $B t$ crop and/or their importance to ecosystem processes in a given area (Zeilinger et al., 2010).

Effects of the cultivation of Bt crops on microarthropods

Microarthropods, including isopods (pillbugs, woodlice), collembolans (springtails), and mites are other organisms in the soil ecosystem that may be affected by the cultivation of transgenic $B t$ crops (Table 1.8). Their important role in nutrient cycling and degradation of plant material may be affected by changes in the structure, chemistry, and other properties of soil by Cry proteins deposited from transgenic plant biomass or root exudates. Despite being more closely related to target insect pest populations (e.g., Lepidoptera, Coleoptera, Diptera) than the other soil organisms already discussed, most microarthropods, including the woodlouse Porcellio scaber (Escher et al., 2000; Pont and Nentwig, 2005), the pillbugs Armadillidium nastum and Trachelipus rathkii (Clark et al., 2006), and the collembolans Folsomia candida (Sims and Martin, 1997; EPA, 2001b; Bakonyi et al., 2006; Clark and Coats, 2006; Bakonyi et al., 2011), Xenylla griesea (Sims and Martin, 1997), and Protaphorura armata (Heckmann et al., 2006), as well as natural populations of collembolans (Lang et al., 2006; de Vaufleury et al., 2007; Priestley and Brownbridge, 2009), have shown no adverse effects in soil from the cultivation of $B t$ Cry1 Ab maize, consumption of $B t$ Cryl Ab maize plant material, or consumption of purified Cry1 Ab protein in studies in microcosms and in the field. Similarly, there was no negative effect of transgenic Bt cotton expressing Cry1 Ab or Cry1 Ac or Bt potato 
expressing Cry3A on F. candida (Yu et al., 1997; EPA, 2001b) and also no effect of Bt potato on the number of eggs and body length of the mite Oppia nitens (Yu et al., 1997). In laboratory experiments where the orbatid mite, Scheloribates praeincisus, was grown in rearing chambers and fed $B t$ cotton leaves (Bollgard; Cry1Ac), non-Bt cotton leaves, and the $B t$ biopesticide Dipel ${ }^{\circledR}$ (containing spores of B. thuringiensis var. kurstaki, HD1, coding for the expression of the insecticidal d-endotoxin Cry1Ab), there was no effect on survival or development of adult or immature mites, nor was there an effect on food consumption (Oliveira et al., 2007). Cultivation of $B t$ maize expressing Cry3Bb1 showed no deleterious effects on the numbers of collembolans or mites in natural populations of field soil (Al-Deeb et al., 2003). In a field experiment where litterbags containing plant material from 9 different $B t(\mathrm{Cry} 1 \mathrm{Ab}, \mathrm{Cry} 3 \mathrm{Bb} 1)$ and non- $B t$ maize lines were buried in soil, there was no difference in decomposer communities (Collembola, Acari, and 12 taxa of other arthropods) or degradation rate between the different cultivars (Honemann et al., 2008). When the effects of individual purified proteins were tested (Cry1Ab, Cry2A, Cry1Ac, Cry3A) in a feeding study over 21 days in Petri dish microcosms, there were no toxic effects detected on the survival or reproduction of $F$. candida or X. griesea (Sims and Martin, 1997) and no effects of purified Cry2A on P. saber (Sims, 1997).

Only a few studies have reported negative effects of $B t$ proteins on microarthropods: Wandeler et al. (2002) found that $P$. scaber fed significantly less on $B t$ maize expressing $\mathrm{Cry} 1 \mathrm{Ab}$ than on non- $B t$ maize during a 20 -day feeding trial in the laboratory in soil 
microcosms; Griffiths et al. (2006) reported lower collembolan abundance and higher mite populations in soil cultivated with $B t$ maize expressing CrylAb than in soil cultivated with non- $B t$ maize in the laboratory; and Bakonyi et al. (2006) found that there were species-specific effects in feeding preference when the collembolans, $F$. candida, Heteromurus nitidus and Sinella coeca, were fed dried leaves of Bt (Cry1 Ab) or non-Bt maize in laboratory feeding tests $-F$. candida preferred non- $B t$ maize as a food source over $B t$ maize but there was no difference in feeding preference for $H$. nitidus and $S$. coeca. Cortet et al. (2007) detected a minor negative effect of $B t$ maize (Cry1 Ab) cultivation on microarthropod abundance (mites and collembolans) in high-clay soils in field trials; however, agricultural practices had a comparable or greater effect on microarthropod abundance than the Bt crop. Debekijak et al. (2007) also reported a lower abundance of collembolans in field soil cultivated in $B t$ maize (Cry1 Ab), but only at one site and only in early fall; no difference in functional groups of Collembola was detected.

Research recommendations: Effects of the cultivation of $B t$ crops on microarthropods In general, cultivation of transgenic $B t$ crops, $B t$ plant biomass, or purified $B t$ proteins, including Cry1Ab, Cry1Ac, and Cry3Bb1, have had little to no effect on most microarthropods tested. Where effects have been reported, they were minor, and the microarthropods were often more affected by temporal differences in sampling time, agricultural practices, plant varietal differences, or other biotic and abiotic factors not 
related to a particular $B t$ protein. Although most studies have not shown a consistent pattern of $B t$ effects on microarthropods, longer-term field experiments would improve the current understanding $B t$ effects on the abundance and diversity of microarthropods in the soil environment (e.g., Theissen and Russell, 2009). Future studies should focus on multitrophic level interactions and continue to evaluate the effects of new and different types of $B t$ crops on nontarget microarthropods in the soil.

Effects of the cultivation of Bt crops on nontarget Lepidopteran larvae and aquatic insects

Although most insects are not generally classified as soil organisms per se, many insects (e.g., Lepidoptera, Coleoptera, and Hymenoptera) have a larval stage that lives in or feeds in close proximity to $B t$ plant leaves and other residues, $B t$ pollen, and/or Cry proteins in soil. Thus, it is possible for nontarget insects, including pollinators, prey and predator species, and biocontrol agents, to be affected by the cultivation of $B t$ crops, even if they do not feed on the GM crop directly. As the number of studies evaluating the effects of $B t$ crops on nontarget insects are too numerous to review in this chapter, only the nontarget effects of the cultivation of $B t$ crops on selected species of Lepidoptera and aquatic insects will be summarized here (Table 1.9). More information on the nontarget effects of the cultivation of $B t$ crops on a variety of insect species can be found in several reviews including (Romeis et al., 2006; Marvier et al., 2007; Thies and Devare, 2007; 
Duan et al., 2008; Wolfenbarger et al., 2008; Lovei et al., 2009; Lundgren et al., 2009; Lang and Otto, 2010; Gatehouse et al., 2011).

Many of the studies evaluating the effects of the cultivation of $B t$ crops on nontarget insects have been conducted on Lepidoptera, primarily the larvae of Monarch (Danaus plexippus) and Swallowtail (Papilio sp.) butterflies. Larvae of the Monarch butterfly, for example, do not feed on $B t$ crop plants, but their primary food source (milkweed) often grows in or near agricultural fields where Bt pollen deposition on milkweed leaves could occur (Lang et al., 2004). Losey et al. (1999) reported that Monarch caterpillars fed pollen from $B t$ maize (Event 176, Cry1 Ab) in laboratory studies ate less, grew more slowly, had higher mortality than larvae fed non-Bt maize pollen, and cautioned that $B t$ crop cultivation may have serious unintended consequences to nontarget Lepidoptera in the environment. However, critics pointed out that the authors neglected to record the amount of $B t$ corn pollen deposited on the milkweed leaves fed to the Monarch caterpillars and argued that the results reported in laboratory studies were unlikely to be observed under field conditions (reviewed and discussed in Shelton and Sears, 2001). Since then, additional studies have evaluated the effects of $B t$ pollen on nontarget Lepidoptera under both laboratory and field conditions (e.g., Jesse and Obrycki, 2000; Wraight et al., 2000; Hellmich et al., 2001; Sears et al., 2001; Stanley-Horn et al., 2001; Tschenn et al., 2001; Zangerl et al., 2001; reviewed in Gatehouse et al., 2002; Anderson et al., 2004; Candolfi et al., 2004; Dively et al., 2004; Anderson et al., 2005; Gathmann et 
al., 2006; Lang and Vojtech 2006; Prasifka et al., 2007), reviewed by (Sears et al., 2001; Shelton and Sears, 2001; Sears, 2004; Lang and Otto, 2010).

Wind pollinated crops, such as $B t$ maize, have a greater potential for nontarget effects of pollen deposition on Lepidopteran larvae (and other insects) than non-wind pollinated GM crops such as $B t$ potato, $B t$ cotton, or $B t$ canola, and thus have been the focus of many risk assessment studies. In an analysis of 20 peer-reviewed publications (16 laboratory feeding studies with purified $B t$ toxin, $B t$ maize pollen, and/or $B t$ maize anthers in an artificial diet or on leaf disks, whole leaves, or whole plants, and 7 fieldbased studies using unfenced host plants, field cages, or natural conditions), Lang and Otto (2010) found adverse effects of $B t$ maize and/or $B t$ protein on nontarget Lepidopteran larvae (Danaus plexippus, Papilio polyxenes, Papilio machaon, Pieris rapae, Pieris brassicae, Pseudozizeeria maha, Inachis io, Euchaetes pernyi, Plutella xylostella, and/or Galleria mellonella) in 52\% of laboratory-based and in $21 \%$ of fieldbased studies. The majority of these studies were based in the USA and focused on nontarget effects of $B t$ maize pollen (primarily from Events MON810, Bt 176, Bt 11) on Monarch butterfly larvae. The effects most often studied in these experiments were on larval body mass, survival, and developmental time, whereas effects on adult Lepidoptera were rarely examined (Lang and Otto, 2010). The results of each study appeared to depend primarily on the methodology used, the $B t$ cultivar, and the amount of Cry protein expressed in the pollen of each Bt cultivar. Although several field-based studies found no 
effect of $B t$ crop cultivation on nontarget Lepidoptera (black swallowtail and Monarch) (e.g., Wraight et al., 2000; reviewed in Sears et al., 2001; Tschenn et al., 2001; Anderson et al., 2004), the results of Losey et al. (1999) were supported by those of other studies that demonstrated significant adverse effects on swallowtail and/or Monarch caterpillars in the presence of Bt pollen in the field (e.g., Jesse and Obrycki, 2000; Stanley-Horn et al., 2001; Zangerl et al., 2001; Dively et al., 2004) and in laboratory feeding studies (e.g., Hellmich et al., 2001; Anderson et al., 2004; Anderson et al., 2005; Prasifka et al., 2007). Most studies have shown that pollen from Bt maize Event 176 had the most lethal and sublethal effects (e.g., mortality, growth rate, body weight, development time) on larvae of swallowtail and Monarch butterflies, whereas Bt maize Events $B t 11$ and MON810 had negligible effects on the larvae. Bt maize Event 176 was shown to have more than 50 times the level of CrylAb found in Bt maize Events MON810 and Bt 11 (EPA, 2001a). When the EPA registration of $B t$ maize Event 176 expired in 2001, it was not renewed by the producing seed companies, primarily as a result of concerns about pest resistance, and is, thus, no longer grown in the United States (EPA, 2011).

Insect larvae in aquatic ecosystems may also be impacted by the cultivation of $B t$ crops as a result of the transport and accumulation of transgenic $B t$ biomass in rivers and streams (Douville et al., 2007; Rosi-Marshall et al., 2007; Prihoda and Coats, 2008; Swan et al., 2009; Chambers et al., 2010; Jensen et al., 2010; Tank et al., 2010; Wolt and Peterson, 2010; reviewed by Viktorov, 2011). Many rivers and streams run through the 
Midwestern part of the United States, where most of the nation's corn crop is grown, and $B t$ plant biomass is often deposited into aquatic ecosystems by wind and transported downstream where it can accumulate in the bends or edges of the waterways (Tank et al., 2010) and in sediments (Douville et al., 2007). Invertebrate consumers are abundant in agricultural streams (e.g., Moore and Palmer, 2005; Menninger and Palmer, 2007) and are likely to feed directly on decaying plant material, including $B t$ maize. Tank et al. (2010) reported that $86 \%$ of 217 stream sites surveyed in Indiana, USA, in 2007, when $75 \%$ of all maize cultivated in the USA was engineered to express single or stacked combinations of insecticidal and/or herbicide resistance traits (USDA, 2010), contained plant material from maize, including leaves, cobs, husks, and stalks. Thus, it was not surprising that Cry1 Ab protein was detected in the water column at $23 \%$ of these sites. What was unexpected, however, was that CrylAb was detected in streams even in areas where no maize fields were present within 500 meters, indicating that $B t$ toxins may be more widely distributed in aquatic ecosystems than previously realized. As these stream sites were sampled 6 months after the corn fields were harvested, levels of CrylAb (and other $B t$ proteins) would probably be even higher immediately following harvest when plant biomass is fresh. Douville et al. (2007) reported that CrylAb gene from Bt maize cultivation could be detected in surface water and sediments for more than 21 and 40 days, respectively. The Cry1 Ab protein was found in higher concentrations in the clay and sand-rich sediments than in surface water, and could still be detected at least $82 \mathrm{~km}$ 
downstream from the $B t$ maize plot, although the $B t$ concentration tended to decrease the farther away from the plot it was detected.

Some of the first evidence of the potential sensitivity of some aquatic insects to $B t$ biomass was reported in a laboratory experiment whereby caddisfly larvae fed $B t$ maize residue grew less and had higher mortality than larvae fed non-Bt maize residue (RosiMarshall et al., 2007). Caddisflies are closely related to target pests, and the larvae are an important food source for fish and other marine life. Chambers et al. (2010) reported that the aquatic leaf shredder, Lepidostoma liba, grew more slowly when fed Bt maize residue in laboratory trials. However, no negative effects were detected on the abundance or diversity of natural populations of nontarget aquatic invertebrates including Coleoptera, Diptera, Ephemeroptera, Hemiptera, Odonata, Plecoptera, and Trichoptera in a field study, possibly because the $B t$ plant biomass was already highly degraded at the time of sampling (Chambers et al., 2010). In a laboratory feeding study, differences in the composition of plant tissue from different maize cultivars, rather than a direct toxic effect of Cry protein in the $B t$ maize varieties, were found to affect the growth and survivorship of nontarget aquatic detritivores (e.g., leaf chewing shredders), including crane fly larvae and the aquatic isopod, Caecidotia communis (Jensen et al., 2010). Until recently, most risk assessment research has focused on the nontarget effects of $B t$ crops in the terrestrial environment. Thus, it is largely unknown what long-term effects, if any, could be expected on nontarget aquatic invertebrates and detritivores that are exposed to $B t$ plant 
material, which appears to be widely dispersed throughout aquatic ecosystems.

Research recommendations: Effects of the cultivation of $B t$ crops on nontarget Lepidopteran larvae and aquatic insects

Although it has been demonstrated that some nontarget insects (e.g., Lepidoptera) can be negatively impacted by certain $B t$ cultivars, particularly those that express high levels of the Cry proteins in pollen, root exudates, and plant biomass, further studies are needed to determine the long-term health and reproductive success of nontarget insect larvae that feed on naturally-deposited $B t$ pollen and other plant residues in the field. Because most $B t$ crops developed thus far are not wind-pollinated, and would therefore have minimal impacts of pollen drift on nontarget insect larvae, it will be important to evaluate the impact of $B t$ plants that are wind pollinated and/or are cultivated in close proximity to the primary food sources of nontarget insects. Moreover, the scope of future biotech risk assessment research should be broadened to assess the impacts of $B t$ crops on a variety of insect species in different parts of the world, as many risk assessment studies, to date, have focused primarily on effects on nontarget insect larvae in the USA.

Continuing to monitor and test for effects of the cultivation of $B t$ crops on nontarget aquatic insects is also important, particularly as the deposition of $B t$ plant biomass into streams and rivers is common throughout $B t$ crop-growing regions of the USA. Because Cry proteins from $B t$ maize have been detected in streams at least up to $82 \mathrm{~km}$ 
downstream, nontarget insects such as caddisfly larvae and other aquatic invertebrates that serve as an important food source for fish should be monitored for nontarget effects of different types of $B t$ proteins, and over multiple distances away from the source. In some cases, there may even be a continual source of $B t$ residue deposition into aquatic ecosystems, as more and different types of $B t$ crops are cultivated near waterways, and $B t$ plant debris enters aquatic ecosystems at different times throughout the growing season. If further research demonstrates negative effects of $B t$ proteins, the cultivation of $B t$ crops, and/or Bt biomass on nontarget insect larvae in aquatic and/or terrestrial ecosystems, it may be possible to use these data to develop new conditions that can be imposed upon registration of $B t$ products and to establish new scientific evaluation protocols that would minimize the environmental impacts of different types of $B t$ crops in both types of systems.

\section{Gene Escape/Introgression}

Gene flow and introgression of $B t$ crops has been a concern in both the agriculture and scientific communities since transgenic crops were commercially released, so much so that the planting of GM PIP crops in close proximity to SCWR in the USA is regulated by the EPA (for more details, see 'How Bt and other GM plants are regulated in the USA' earlier in this chapter). Although the regulatory agencies in the USA have a fairly rigorous oversight role in protecting the interests of farmers, consumers, seed companies, 
and the environment, other countries, particularly those with small economies, may not be able to enforce the types of regulations that the USA has designed to minimize environmental impact, especially with regard to containment and/or separation of GM products (produce, grain, seed), setting aside refuge areas to minimize the development of pest resistance, creating buffers to limit gene flow, and/or monitoring for nontarget effects in the environment. This potential lack of enforcement and/or regulations to monitor $B t$ (and other transgenic) crops is of concern, as almost half of the global hectarage of GM crops is grown in developing nations where this level of oversight may not be feasible. It is, therefore, imperative that GM crops be carefully evaluated for nontarget effects and the potential for gene-flow under a variety of environmental and experimental scenarios, even after they have been approved for commercial use in the USA.

Examples of gene-flow from transgenic crops to SCWR are becoming more prevalent, and as more and different types of transgenic crops are introduced each year, gene-flow between them will become inevitable (Snow, 2002). In one of the first studies to examine 'transgene escape', Quist and Chapela (2001) reported that genes from Bt maize had introgressed into ancient landraces of traditional maize in Oaxaca, Mexico. This was despite a six-year moratorium, implemented in 1998, on the cultivation of GM maize in Mexico. A follow-up study, conducted in 2005, found no traces of the genetically engineered traits (specifically the cauliflower mosaic virus promoter) in any 
of the Mexican maize samples tested, but rather than attempting to disprove the findings of Quist and Chapela, Ortiz-Garcia et al. (2005) concluded that the moratorium, as well as increased education among the farmers, likely led to the lack of genetic contamination detected in their study. Pineyro-Nelson et al. (2009) employed new molecular techniques to confirm the presence of transgenes in three of 23 localities sampled in Oaxaca in 2001 and demonstrated that the persistence or re-introduction of transgenes from Bt maize continued up until at least 2004. It is not clear how changes in plant physiology due to flow of transgenes might influence rhizosphere ecology, if at all, but as new and different types of GM crops are developed, it is important to consider the potential impacts of gene flow on nontarget soil organisms in different agricultural and non-agricultural environments.

Escape of transgenes has also been reported in other GM crops. In Oregon, for example, EPA researchers discovered gene escape (CP4 EPSPS protein and the corresponding transgene) from glyphosate-resistant creeping bentgrass (Agrostis stolonifera) grown in USDA approved Monsanto-Scotts test plots (Reichman et al., 2006). The researchers found the transgenes incorporated into resident populations of compatible Agrostis species up to $3.8 \mathrm{~km}$ away from the USDA-APHIS permitted field test site, supporting a previous study where gene flow between glyphosate-resistant creeping bentgrass and sentinel and resident plants occurred up to $21 \mathrm{~km}$ and $14 \mathrm{~km}$ away, respectively, from the perimeter of the GM bentgrass test plot (Watrud et al., 
2004). These studies provide evidence that novel traits can spread to wild-type plants and related species over much greater distances than previously realized.

Gene flow and introgression of some GM traits may also affect plant population or plant-soil dynamics that may indirectly aid in the spread of invasive species via the alteration of the soil community. When the effect of glyphosate drift (10\% application rate of Roundup Ready $\left.{ }^{\circledR}\right)$ was evaluated on mixed-species mesocosm communities consisting of GM glyphosate resistant Brassica, two sexually compatible Brassica relatives, and a selection of annual weeds (Digitaria sanguinalis, Panicum capillare, and Lapsana communis), Watrud et al. (2011) found that crabgrass was the dominant weed in the control treatments and that Brassica dominated in the glyphosate treatments, increasing the incidence of the Roundup ${ }^{\circledR}$ resistance gene in the plant community. When Trifolium incarnatum (Crimson clover) was planted in soil mesocosms that had received glyphosate drift treatments, shoot biomass and AMF colonization were reduced compared with plants grown in mesocosms that received no herbicide treatment (Watrud et al., 2011). These results suggest that glyphosate drift associated with HT GM crops could contribute to the persistence and spread of certain invasive species, such as Brassica sp., which are non-mycorrhizal, and thus could facilitate the process of invasion by altering the mycorrhizal community in the soil over time. Introgression of a $B t$ gene that had a negative impact on AMF (or any other group of soil organisms) might be expected to have similar effects on the soil ecosystem, but this remains to be seen. 


\section{Can Bt crops have a role in sustainable agroecosystems?}

It has been proposed that $B t$ crops could contribute to agricultural sustainability by reducing the amounts of chemical insecticides that are usually applied in conventional agricultural systems, improving yield in areas where insect-control measures are limited, and minimizing the negative effects to soil structure associated with tillage, as $B t$ genes are often also stacked with herbicide-tolerant traits. By contributing to reduced insecticide usage, $B t$ crops could confer benefits to farm workers and the environment, and because many $B t$ cultivars also include herbicide tolerance traits, conservation tillage measures could be employed to reduce the effects of tillage on soil organisms and help minimize loss of soil from erosion. The potential environmental benefits of $B t$ crops may perhaps be best illustrated by $B t$ cotton. Historically, $25 \%$ of all insecticides used in agriculture were applied to cotton - more than to any other crop (James, 2010). By planting $B t$ cotton that contains its own insecticide targeted against the cotton bollworm, the environment has been spared from pollution with thousands of pounds of broadspectrum insecticides each year. The EPA reported that $B t$ cotton reduced insecticide use by nearly one million gallons in 1999 alone and saved farmers nearly \$500/acre in chemical costs. The high level of confidence that many farmers have in this type of crop biotechnology is reflected in the large amount of land dedicated to GM crop production in the USA: in $2010,93 \%$ of the cotton crop and $86 \%$ of the corn crop in the USA was 
genetically engineered (USDA, 2010). Bt corn has also been shown to offer communal benefits, whereby non- $B t$ plants grown in close proximity to $B t$ fields also benefit from reduced pest damage through a 'halo effect' on the target pest population (Alstad and Andow, 1996; Hutchison et al., 2010). Moreover, because the $B t$ protein expressed in crop plants have high specificity to certain insect groups (i.e., Lepidoptera, Coleoptera), the Cry proteins are not likely to have direct toxic effects on nontarget organisms (with a few exceptions). There is also the potential for increased yield of $B t$ crops (when compared with crops without insect protection), which may help to reduce land area required for agricultural production. It remains to be seen if this, indeed, will be the case, however, as high-yielding crop varieties can also be developed through conventional breeding methods, and the use of cultivars adapted to particular agricultural regions can also improve yields.

The relatively rapid and widespread commercialization of transgenic crop technology, however, has contributed to a certain level of mistrust and suspicion by the general public, particularly in countries outside of the USA. The public perception is that GM crops are being rapidly adopted by farmers throughout the world without a complete understanding of the long-term environmental impacts. Even in the USA, 100 times more resources are invested in developing transgenic crops than are spent on risk assessment and monitoring for nontarget effects after their commercial release (Thies and Devare, 2007). While $B t$ crops may help to improve the sustainability of conventional agricultural 
systems by reducing insecticide usage, they may not be of benefit in agricultural systems where long-term sustainability is the goal (i.e., organic farming systems and/or low-input farming systems) as they offer few, if any, benefits to enhancing soil fertility and may even have negative effects on fungal symbionts that are essential for nutrient uptake in low-input systems. Moreover, caution should be exercised when cultivating certain types of GM crops in regions where the precautions necessary to prevent gene flow and insect resistance may not be practical. Gene flow may be of particular concern in tropical areas where the wild relatives of many agricultural crops are endemic and the hectarage dedicated to the cultivation of GM crops is increasing.

\section{Conclusions and future directions}

To date, risk assessments of GM crops indicate that there are few to no consistent negative effects of $B t$ protein, the cultivation of $B t$ plants, and/or $B t$ plant residues on most of the soil bacteria, saprotrophic and pathogenic fungi, protozoa, earthworms, and microarthropods evaluated. When significant effects have been detected, they have often been minimal and/or transitory, making their ecological significance difficult to gauge. While the research conducted thus far is by no means exhaustive, it is encouraging to note that few negative effects have been detected on soil organisms, despite the widespread and long-term cultivation of $B t$ crops. The soil organisms that appear to be most sensitive to $B t$ crop cultivation include AMF, nematodes, and nontarget insect 
larvae that live and/or feed in soil in close proximity to areas cultivated with $B t$ crops.

As nontarget effects cannot always be attributed to a particular Cry protein, risk assessment research should include investigations to identify genetic alterations that produce a change in the physiology or tissue composition of the plant. A genetic insertion that results in a change in plant root exudates, for example, could influence species composition in the rhizosphere without being an effect of the $B t$ protein itself.

Internationally, more research funding should be directed towards risk assessment of GM plants in the areas of fungal ecology, nematode abundance and diversity, and insect ecology in both terrestrial and aquatic ecosystems. Moreover, each new GM plant line should be tested for effects on nontarget organisms under a variety of environmental and experimental conditions, as results of previous studies varied depending on biotic and abiotic factors that may or may not be associated with the expression of $B t$ proteins. Research should also continue investigate nontarget effects on symbionts that improve plant performance (e.g., nitrogen-fixing bacteria, AMF), as these are soil organisms that may be most sensitive to $B t$ proteins or to cultivation of $B t$ crops because of their dependence on a plant host. A focus on long-term field experiments and collaborative research efforts between soil ecologists, agroecologists, microbial ecologists, and others, will help to understand better the long-term effects of the cultivation of $B t$ crops on multitrophic level interactions (e.g., Hilbeck et al., 1999; Groot and Dicke, 2002; Guo et al., 2008) and ecosystem functioning. Future investigations should incorporate the use of 
molecular methods for the identification and quantification of different trophic groups of soil microorganisms, as plate counts and other culturing methods are generally not sufficiently specific. As more and different types of $B t$ crops are developed, it will be important to evaluate the effects of each genetic insertion event on a variety of nontarget organisms under different environmental and experimental scenarios. Allocating sufficient resources to post-release monitoring will also be important for mitigating any potential negative effects of the cultivation of GM crops on nontarget organisms in both terrestrial and aquatic ecosystems. 


\section{Chapter 2: The influence of fertilizer level and spore density on arbuscular mycorrhizal colonization of transgenic Bt 11 maize (Zea mays) in experimental microcosms}

Published as: Cheeke, T. E., Pace, B. A., Rosenstiel, T. N., and Mitchell B. Cruzan. 2011. The influence of fertilizer level and spore density on arbuscular mycorrhizal colonization of transgenic Bt 11 maize (Zea mays) in experimental microcosms. FEMS Microbiology Ecology. 75: 304-312.

\section{Abstract}

Crop plants genetically modified for the expression of Bacillus thuringiensis $(B t)$ insecticidal toxins have broad appeal for reducing insect damage in agricultural systems, yet questions remain about the impact of $B t$ plants on symbiotic soil organisms. Here, arbuscular mycorrhizal fungal (AMF) colonization of transgenic maize isoline $B t 11$ (expressing Cryl $\mathrm{Ab}$ ) and its non-Bt parental line (Providence) was evaluated under different fertilizer level and spore density scenarios. In a three-way factorial design, $B t 11$ and non-Bt maize were inoculated with 0,40 , or 80 spores of Glomus mosseae and treated weekly with 'No' $\left(0 \mathrm{~g} \mathrm{~L}^{-1}\right)$, 'Low' $\left(0.23 \mathrm{~g} \mathrm{~L}^{-1}\right)$, or 'High' $\left(1.87 \mathrm{~g} \mathrm{~L}^{-1}\right)$ levels of a complete fertilizer and grown for 60 days in a greenhouse. While no difference in AMF colonization was detected between the $B t 11$ and P maize cultivars in the lower spore/higher fertilizer treatments, microcosm experiments demonstrated a significant reduction in AMF colonization in $B t 11$ maize roots in the 80 spore treatments when fertilizer was limited. These results confirm previous work indicating an altered relationship between this $B t 11$ maize isoline and AMF and demonstrate that the 
magnitude of this response is strongly dependent on both nutrient supply and AMF spore inoculation level.

\section{Introduction}

Since the commercial introduction of genetically modified crops in 1996, the acreage dedicated to transgenic crop production has risen each year worldwide (James, 2010). Currently $80 \%$ of all maize grown in the United States and over $25 \%$ of the maize cultivated globally is genetically modified to express herbicide resistance, insecticidal properties, or a combination of stacked traits (USDA, 2008; James, 2010). Insectresistant $B t$ maize, one of the most widely cultivated transgenic crops, has been genetically engineered to express insecticidal toxins derived from the spore-forming soil bacterium $B t$. The insecticidal crystal proteins (Cry proteins) in Bt crops are characterized by a high specificity toward certain insect groups [e.g. CrylAb is only toxic for Lepidoptera such as the European corn borer (Ostrinia nubilalis)] and do not appear to have a direct effect on nontarget organisms in the soil environment (e.g., Saxena \& Stotzky, 2001; Ferreira et al., 2003; Baumgarte \& Tebbe, 2005; de Vaufleury et al., 2007; reviewed by Thies \& Devare, 2007; reviewed by Icoz \& Stotzky, 2008). However, some studies have reported that certain isolines of $B t$ maize expressing Cry1 $\mathrm{Ab}(B t 11$ and $B t$ 176) are poorly colonized by arbuscular mycorrhizal fungi (AMF) (Turrini et al., 2004; Castaldini et al., 2005). While plants vary naturally in their AMF hosting ability 
(Newman \& Reddell, 1987; Trappe, 1987), genetically engineering plants may, in some cases, alter their relationship with AMF. Because AMF are obligate symbionts that require a plant host for nutrition and reproduction, they may be more sensitive to changes in the physiology of the host plant than other soil-dwelling microorganisms and should be carefully evaluated for nontarget impacts by transgenic $B t$ plants.

AMF are an important component of the soil ecosystem and can improve plant nutrient acquisition in the absence of synthetic chemical fertilizers and in other low nutrient environments (e.g., Smith \& Read, 1997; Galvez et al., 2001; Gosling et al., 2006; Lekberg et al., 2008; Sheng et al., 2008). To date, the effects of Bt crop plants on AMF colonization are inconsistent. While a few studies have shown reduced colonization in some Bt maize isolines expressing Cry1 Ab (Turrini et al., 2004; Castaldini et al., 2005), other studies have reported no difference in AMF colonization of Bt maize expressing the same protein (MON810, Cry1Ab) (de Vaufleury et al., 2007) or Bt cotton expressing other Bt proteins (Cry1Ac and Cry2Ab) (Knox et al., 2008). As these studies differ greatly with respect to sampling time, fertilizer level, transgenic line, Cry protein, and the number and type of spores used, it is difficult to identify the primary factors influencing the patterns of AMF colonization reported for the different $B t$ cultivars. It is possible that the reductions in AMF colonization observed in certain $B t$ isolines expressing Cry1 Ab are simply due to underlying differences in experimental conditions, or from an indirect effect of the genetic insertion, rather than a direct effect of the 
Cry1 Ab protein on soil fungi. As nutrient availability and spore inoculation level are thought to be two key environmental factors influencing AMF infection (e.g., Smith \& Read, 1997), differences in fertilizer level and spore density across experimental designs may help to explain the diversity of results observed to date.

In this study, fertilizer level and spore inoculation level were manipulated to determine the ecological conditions that may lead to a difference in AMF colonization reported between $B t 11$ maize and its parental cultivar. Here, Bt maize (Zea mays, event Bt 11, expressing Cryl $\mathrm{Ab}$ ) and its non-Bt parental line (Providence: hereafter referred to as P) were evaluated for AMF colonization by Glomus mosseae under three different fertilizer level and spore inoculation level scenarios. These microcosm experiments were conducted in a greenhouse using autoclaved soil to examine the specific effects of fertilizer level and spore density while controlling for other microbial components that might influence AMF symbiosis. Initial height of each seedling was recorded at the time of transplanting and growth responses (root biomass, shoot biomass, and chlorophyll content) were recorded after 60 days to determine whether plants with higher levels of AMF colonization exhibited any growth benefits as a result of the symbiosis. It was hypothesized that the greatest difference in AMF colonization between the $B t 11$ and $\mathrm{P}$ isolines would be observed when fertilizer was limited and spore inoculation level was high as this is when the level of AMF infection would be expected to be highest in both cultivars (e.g., Smith \& Read, 1997), and that plants with the highest level of AMF 
colonization would have the greatest biomass and chlorophyll content at the end of the experiment.

\section{Materials and Methods}

\section{Bt maize cultivar}

Zea mays (ATTRIBUTE, triple sweet hybrid sweet corn, isoline Bt 11: BC0805) and its non- $B t$ near-isogenic parental line (P) were obtained from Syngenta Seeds Inc. (Boise, ID). The Bt 11 transgene was backcrossed into one of the parents of Providence to create the variety BC0805 (personal communication, M.V. Mason, Syngenta Seeds, Inc.). The Bt 11 cultivar was transformed using the plasmid pZ01502 (containing Cry1 Ab, pat, and amp genes) to express the Cry1 Ab protein of Bt (EPA, 2007). This Bt 11 containing inbred is an approximate isoline with the non- $B t$ parent (personal communication, Mason, 2010). Isoline $B t 11$ was used in this study as it has been one of the most commonly planted $B t$ maize isolines globally and has been used in previous risk assessment studies (Turrini et al., 2004; Castaldini et al., 2005).

Mycorrhizal fungus

The mycorrhizal fungus culture Glomus mosseae CA210 (pure, sonicated spores) was obtained from the International Culture Collection of Vesicular Arbuscular Mycorrhizal Fungi (INVAM). Glomus mosseae was chosen as it is a ubiquitous, generalist AMF species found in many agroecosystems (e.g., Smith \& Read, 1997; Avio et al., 2009; 
Rosendahl et al., 2009) and has been used in other experiments investigating nontarget effects of Bt maize on AMF (Turrini et al., 2004; Castaldini et al., 2005). The higher spore inoculation level of 80 spores per root system used in this study was chosen as it is similar to the spore density in the root zone of plants found in local agricultural soils (Vancouver, WA; Cheeke, unpublished data).

Plant cultivation and spore application

Seeds of Bt 11 and P maize were surface sterilized in a $10 \%$ bleach solution before being germinated in sterile sand. After approximately three weeks, seedlings with similar sized roots and shoots were selected for transplanting and initial heights were recorded. Roots were rinsed with tap water to remove sand particles and each root system was directly inoculated with a pure culture of 0 (uninoculated controls), 40, or 80 spores of $G$. mosseae. After inoculation, Bt 11 and P maize seedlings were planted into 4 L pots containing autoclaved Whitney Farms Premium Potting Soil (aged and processed softwood bark and sawdust, sphagnum peat moss, pumice, composted animal manure; Scotts Company, LLC). Growth conditions and fertilizer treatments

After transplanting, fertilizer treatments were applied by adding $200 \mathrm{ml}$ of ' $\mathrm{No}$ ' ( $0 \mathrm{~g}$ $\left.\mathrm{L}^{-1}\right)$, 'Low' $\left(0.23 \mathrm{~g} \mathrm{~L}^{-1}\right)$, or 'High' $\left(1.87 \mathrm{~g} \mathrm{~L}^{-1}\right)$ levels of Peter's Professional All Purpose Plant Food 24-8-16 (St Louis, MO) each week. Plants were grown in the greenhouse from April 2007 to June 2007. Five replicates of each isoline were included for every 
inoculation level and fertilizer treatment for a total of 90 plants in the experiment. To account for microclimatic effects, pots were rotated on the greenhouse bench each week using a randomization key. The daytime temperatures in the greenhouse were between $27^{\circ} \mathrm{C}$ and $32{ }^{\circ} \mathrm{C}$ and nighttime temperatures were between $20^{\circ} \mathrm{C}$ and $27^{\circ} \mathrm{C}$, which reflect growing temperatures of many corn-growing regions in the United States. Photoperiod was from 6:00 to 20:00 every day, supplied via metal halide lights and natural sunlight. Humidity varied between $50 \%$ and $70 \%$ throughout the growing period.

\section{Mycorrhizal fungus colonization assessment}

Plants were destructively harvested 60 days after inoculation when the plants were in a period of active growth (with an average of seven live leaves/plant) but before ear production. The 60-day harvest period was chosen based on preliminary experiments (Cheeke, unpublished results) and previous risk assessment studies (Turrini et al., 2004; Castaldini et al., 2005; de Vaufleury et al., 2007). At harvest, roots were rinsed in tap water to remove soil particles. An equivalent amount of cut samples were taken across multiple locations of the root system of each plant and were placed in histocassettes (VWR, West Chester, PA) for processing. Roots were cleared using $10 \% \mathrm{KOH}$, neutralized in $2 \%$ aqueous $\mathrm{HCl}$, and stained with a $0.05 \%$ Trypan Blue solution to visualize fungal structures (Phillips \& Hayman, 1970). Stained roots were cut into approximately $1 \mathrm{~cm}$ segments and mounted in lactoglycerol on microscope slides. At least $50 \mathrm{~cm}$ of roots from each maize plant were assayed for mycorrhizal fungus 
colonization using the slide-intersect method (McGonigle et al., 1990). The presence/absence of arbuscules, hyphae, and vesicles observed per 100 root intersects was recorded.

\section{Plant biomass}

Plant height and leaf number were recorded at the time of transplanting, on day 30 and again on day 60. After root samples had been collected for AMF assessment, the shoots and roots were separated and dried for at least 48 hours at $60^{\circ} \mathrm{C}$ to collect root and shoot biomass data.

\section{Chlorophyll analysis}

The effect of fertilizer treatment in $B t 11$ and $\mathrm{P}$ maize plants was evaluated by quantifying leaf chlorophyll content (Porra et al., 1989). Leaf chlorophyll (Chl) content was determined using standard spectrophotometeric methods (Shimadzu 1201) (Porra, 2002). Leaf cores were taken on the day of harvest from the third leaf up from the bottom of live plants with a \#10 brass corer and frozen at $-80{ }^{\circ} \mathrm{C}$ until analysis. Chlorophylls ( $a$ and $b$ ) were assayed by solvent extraction in buffered $80 \%$ aqueous acetone using the simultaneous equations of Porra et al. (1989). Chlorophyll values were expressed in $\mu \mathrm{mol}$ $\mathrm{m}^{-2}$ leaf area.

Data analysis

The effects of fertilizer level and spore inoculation treatment on AMF colonization percentage data were assessed by ANOVA after arcsin square root transformation. Data 
were analyzed in a three-way ANOVA with uninoculated controls removed from the analysis (no AMF colonization was detected in the uninoculated plants). Fixed effects in this analysis were cultivar (Bt 11 or P), fertilizer level, and spore inoculation level with initial height, root biomass, and shoot biomass included as covariates.

Plant growth responses (total biomass, root biomass, shoot biomass, root/shoot ratio, and chlorophyll content) were analyzed using both two- and three-way ANOVAs using the GLM procedure of SAS. In the two-way ANOVA, cultivar and fertilizer level were entered as fixed effects, and AMF colonization frequencies (entered as a covariate) replaced the spore inoculation level (only the 40- and 80-spore levels were used). AMF colonization was used in this analysis instead of spore inoculation level because actual colonization frequencies varied substantially within the spore inoculation level treatments. In the three-way ANOVA models, the AMF colonization covariate was dropped from the analysis to test the effects of inoculation level (0-, 40-, and 80-spore treatments) independent of the AMF colonization. Fixed effects in the three-way ANOVA models were cultivar, fertilizer level, and spore inoculation level; covariates included initial height, root biomass, and shoot biomass. Plant growth variables (root, shoot and total biomass, and initial height) were log transformed to improve normality. Other response variables (chlorophyll content and root/shoot ratio) were approximately normal without transformation. Differences among individual means for fertilizer and spore treatments were determined using Tukey's multiple range tests for ANOVA 
analyses for each fertilizer or spore treatment level. The difference in the slopes for the relationship between growth and AMF colonization for the two cultivars was tested with the AMF x cultivar interaction (heterogeneity of slopes test). All analyses were performed using SAS (version 9.1).

\section{Results}

Effects of cultivar, fertilizer level, and spore inoculation level on AMF colonization

The three-way ANOVA of AMF colonization levels in Bt 11 and P maize

demonstrated that cultivar, fertilizer level, spore inoculation level, and cultivar $\mathrm{x}$ fertilizer interactions had significant effects on mycorrhizal colonization (Table 10). The P maize plants had significantly higher levels of AMF colonization in their roots compared to the Bt 11 cultivar in the 80 spore treatments when fertilizer was limited (Fig. 2). High fertilizer levels were associated with lower AMF colonization in both cultivars, and higher spore inoculation levels led to increased mycorrhizal colonization in both maize cultivars (Fig. 2). In the 'No fertilizer, 80 spore' treatment, P plants had nearly three times more AMF in their root systems than the $B t 11$ isoline and in the 'Low fertilizer, 80 spore' treatment, $\mathrm{P}$ plants had nearly seven times more AMF in their roots than $B t 11$ plants (Fig. 2a and b). Overall, plants inoculated with 80 spores of G. mosseae had approximately three times more AMF colonization (mean $=11.55 \%, \pm 3.15)$ than plants inoculated with 40 spores (mean $=3.68 \%, \pm 0.99$; Table 2.1$)$. In the 'High' fertilizer 
treatment very little AMF colonization was observed in either cultivar, even when inoculated with 80 spores of G. mosseae (Fig. 2c). In the 40 spore treatments, no significant difference in AMF colonization was detected between the $B t 11$ and non- $B t$ cultivars, likely because the overall level of AMF colonization was less than $10 \%$ for all three fertilizer levels (Fig. 2). The results for the presence of arbuscules and hyphae per hundred intersects were similar to the results for total AMF colonization (Table 10), and hence only the total AMF colonization data are reported in Fig. 2. No vesicles were observed in any transects analyzed and no AMF colonization was detected in the uninoculated control plants (Fig. 2).

Effects offertilizer level, cultivar, and spore inoculation level on plant growth

There were no significant differences in height or leaf number between the cultivars at the time of harvest, even though the $B t 11$ cultivars were slightly taller at the beginning of the experiment. Mean initial height of $B t 11$ and P cultivars was $20.76 \mathrm{~cm}$ and 18.86 $\mathrm{cm}$, respectively $(\mathrm{F}=5.59, \mathrm{P}=0.0202, \mathrm{df}=1 / 90), 107.24 \mathrm{~cm}$ and $99.91 \mathrm{~cm}$ for the 30 day height $(\mathrm{F}=13.74, \mathrm{P}=0.0004, \mathrm{df}=1 / 83)$, and $122.66 \mathrm{~cm}$ and $120.59 \mathrm{~cm}$ for the 60 day height $(\mathrm{F}=0.84, \mathrm{P}=0.3607, \mathrm{df}=1 / 83)$. The 30 -day mean live leaf numbers for the Bt 11 and $\mathrm{P}$ cultivars were 8.02 and 7.98 , respectively $(\mathrm{F}=0.18, \mathrm{P}=0.6722, \mathrm{df}=1 / 83)$, and 7.36 and 7.47 at 60 days $(F=0.03, P=0.8742, \mathrm{df}=1 / 83)$.

As expected, plants in the high fertilizer treatments had greater total biomass, shoot 
biomass and leaf chlorophyll content at the end of the experiment (Table 11; Fig. 3a and b). Root biomass did not differ across all fertilizer treatments, but within the 'Low' fertilizer treatment, the root biomass of $\mathrm{P}$ plants was significantly higher than the $B t 11$ plants (Table 11; Fig. 3c). Root/shoot ratio was also highest in P plants in the 'Low' fertilizer treatment (Table 11; Fig. 3d). These differences in responses of cultivars for root biomass, root/shoot ratio, and chlorophyll content for the low fertilizer treatment contributed to the significant cultivar $\mathrm{x}$ fertilizer interactions (Tables 11 and 12).

Spore inoculation level had a significant effect on total biomass, root biomass, and chlorophyll content (Table 12). When plants were grown without spores in the 'No' and 'Low' fertilizer treatments, Bt 11 plants had a greater shoot biomass than P plants (Fig. 4a and $b$ ); however, this difference in shoot biomass between the two cultivars was not observed in the 40 and 80 spore 'No' and 'Low' fertilizer treatments (Fig. 4a and b). In the 'Low' and 'High' fertilizer treatments, plants inoculated with 40 spores had a greater root biomass compared to the 0 spore treatment (Fig. $4 \mathrm{~b}$ and c). These inconsistencies in responses in root and shoot biomass across the spore and fertilizer treatments contributed to significant cultivar $\mathrm{x}$ fertilizer $\mathrm{x}$ spore interactions (Table 12; Fig. 4). Overall, plants with no AMF inoculum had higher leaf chlorophyll content at the end of the experiment (Table 12, Fig. 5). Within the 'No fertilizer, 0 spore treatment' Bt 11 plants had a greater leaf chlorophyll content than the P plants but this difference between the two cultivars was not detected at the 40 and 80 spore level (Fig. 5a). 


\section{Discussion}

In this greenhouse study, AMF colonization by the AMF species $G$. mosseae was significantly reduced in transgenic maize isoline $B t 11$ (expressing Cry1 $\mathrm{Ab}$ ) in the 80 spore treatments when fertilizer was limited. No difference in AMF colonization was detected between the Bt 11 and P cultivars in the higher fertilizer and lower spore treatments, highlighting the important role of the soil environment in modulating the interaction between this $B t$ maize isoline and AMF. The differences in mycorrhizal colonization that were observed across treatments demonstrate that the magnitude of the response was strongly dependent on fertilizer and spore inoculation level and suggest that multiple environmental factors should be considered in designing risk assessment studies.

By analyzing AMF colonization in Bt 11 and $\mathrm{P}$ maize under different fertilizer levels and spore densities this study supports previous research demonstrating an altered mycorrhizal status in Bt 11 maize expressing the Cry1Ab protein (Turrini et al., 2004; Castaldini et al., 2005). More importantly, this experiment has shown that under circumstances where AMF would be most likely to colonize and be of benefit to the host (i.e. higher spore inoculation level, low nutrients) the symbiosis remains muted in the $B t$ 11 maize plants. Under the different conditions of fertilization applied in this study, it was clear that application of chemical fertilizer inhibits the establishment of the AMF symbiosis in both $B t 11$ and non-transgenic maize. While the finding that colonization 
level and inoculum potential/fertilization regime are linked is not novel (e.g., Smith \& Read, 1997), here it was used as a way to manipulate the AMF colonization and understand the effects of Bt 11 maize on AMF under a range of environmental conditions. The lack of mycorrhizal structures in the 'High' fertilizer treatment illustrates the plant-regulated, facultative symbiotic relationship between maize plants and AMF; when high levels of fertilizer were available, virtually no AMF colonization was detected in either cultivar, even in the higher spore inoculation level treatment.

Interestingly, higher levels of AMF colonization did not increase biomass in the $B t$ and P lines, nor did plants with higher AMF colonization have correspondingly increased leaf chlorophyll content, which would have suggested an improved nutrient status. The relationship with AMF is known to vary over the lifecycle of the plant, and our study can be best understood as a snapshot of the symbiosis. The plant-AMF symbiosis can range from parasitism to mutualism depending on the life stage of the plant, ecological conditions, or differences in cultivation (Johnson et al., 1997; Hirsch, 2004; Jones \& Smith, 2004). As the plants were harvested before maturity, it is not known how the reduced colonization of AMF in Bt plants might influence yield or leaf chlorophyll content in mature plants. However, higher levels of AMF colonization have been linked to increased yields in several agricultural crops including wheat, sorghum, soybean, green peppers, potatoes (e.g., Karagiannidis \& Hadjisavva-Zinoviadi, 1998; Bressan et al., 2001; Al-Karaki et al., 2004), even when grown in high phosphorus conditions (e.g., 
Douds \& Reider, 2003; reviewed in Hamel \& Strullu, 2006; Douds et al., 2007).

While there is a clear demonstration that the Cry1 Ab protein is expressed in $B t 11$ maize roots (EPA, 2007; reviewed in Icoz \& Stotzky, 2008), there is little evidence that it has a direct effect on AM fungi as contrasting results have been obtained using different $B t$ maize cultivars expressing the same protein (Castaldini et al., 2005; de Vaufleury et al., 2007). This limits the predictive ability of many $B t$ risk assessment studies, as to date the effects cannot directly be linked to the expression of a particular $B t$ protein and can therefore not be extrapolated to other $B t$ cultivars. However, the strong effect of soil fertilizer and spore densities demonstrated here provides some insights for explaining the diversity of results observed in previous studies and identifies some important environmental considerations for future evaluations. Including more $B t$-modified isolines, as well as consideration of plant developmental state may help elucidate the specific effects of different $B t$ proteins and isoline-specific physiological effects on the ability of Bt plants to develop mycorrhizae. 


\section{Chapter 3. Evidence of reduced arbuscular mycorrhizal fungal colonization in multiple lines of $B t$ maize}

Published as: Cheeke, T. E., Rosenstiel, T. N., and Mitchell B. Cruzan. 2012. Evidence of reduced arbuscular mycorrhizal fungal colonization in multiple lines of $B t$ maize. American Journal of Botany. 99(4): 700-707.

\section{Abstract}

- Premise of the study: Insect-resistant Bacillus thuringiensis (Bt) maize is widely cultivated, yet few studies have examined the interaction of symbiotic arbuscular mycorrhizal fungi (AMF) with different lines of $B t$ maize. As obligate symbionts, AMF may be sensitive to genetic changes within a plant host. Previous evaluations of the impact of $B t$ crops on AMF have been inconsistent, and because most studies were conducted under disparate experimental conditions, the results are difficult to compare.

- Methods: We evaluate AMF colonization in nine Bt maize lines, differing in number and type of engineered trait, and five corresponding near-isogenic parental (P) base-hybrids in greenhouse microcosms. Plants were grown in 50\% local agricultural soil with low levels of fertilization, and AMF colonization was evaluated at 60 and 100 days. To test for non-target effects of $B t$ cultivation on AMF colonization in a subsequently planted crop, Glycine max was seeded into soil that had been pre-conditioned for 60 days with $B t$ or P maize. 
- Key results: We found that $B t$ maize had lower levels of AMF colonization in their roots than the non-Bt parental lines. However, reductions in AMF colonization were not related to the expression of a particular Bt protein. There was no difference in AMF colonization in G. max grown in the Bt or P preconditioned soil.

- Conclusions: These findings are the first demonstration of a reduction in AMF colonization in multiple $B t$ maize lines grown under the same experimental conditions and contribute to the growing body of knowledge examining the unanticipated effects of $B t$ crop cultivation on non-target soil organisms.

\section{Introduction}

Genetically modified (GM) crops, engineered to express herbicide-tolerance, insecticidal properties, or a combination of traits, are the most rapidly adopted agricultural biotechnology in recent history (James, 2010). Since their commercial introduction in 1996, the global adoption of GM crop technology has increased ca. 87fold, up from 1.7 million hectares in 1996 to 148 million hectares in 2010 (James, 2010). Insect-resistant maize (Zea mays L.), one of the most widely cultivated GM crops, is engineered to express insecticidal toxins derived from the spore-forming soil bacterium Bacillus thuringiensis (Bt). To date, more than 60 different $B t$ crystal proteins (called 'Cry' proteins) that exhibit a high degree of specificity towards certain insect pests have 
been identified (reviewed in Schnepf et al., 1998; Federici, 2002; Stotzky, 2002; Lee, Saxena, and Stotzky, 2003; Icoz and Stotzky, 2008a; Sanchis, 2011). Bt crops that provide resistance to multiple agricultural pests, as well as confer herbicide-tolerance, have contributed to the popularity of GM crops among farmers worldwide (EPA, 2011). In $2010,86 \%$ of the maize grown in the USA (USDA, 2010) and $26 \%$ of the global biotech hectarage was cultivated in maize genetically modified to express one or more engineered traits (James, 2010). This rapid and widespread adoption of GM crops has led to a dramatic shift in the agricultural landscape over the last 15 years and has raised questions about the impact of insect-resistant $B t$ crops on non-target organisms in the soil environment.

Arbuscular mycorrhizal fungi (AMF) are obligate plant symbionts that have been shown to improve plant nutrient acquisition, especially in low nutrient soil environments (e.g., Galvez et al., 2001; Gosling et al., 2006; Lekberg, Koide, and Twomlow, 2008; Sheng et al., 2008). These symbiotic fungi are ubiquitous in soil and are found in both natural and agroecosystems (Smith and Read, 2008). Because AMF rely on a plant host for nutrition and reproduction, they may be sensitive to changes in the physiology of the host plant, to biochemical changes associated with the $B t$ modification, or to alterations in root exudates released into the rhizosphere. Although $B t$ proteins are expressed in the roots of most $B t$ maize lines (Saxena and Stotzky, 2000; Saxena, Flores, and Stotzky, 2002; reviewed by Icoz and Stotzky, 2008a; Icoz and Stotzky, 2008b; EPA, 2011), the 
evidence that Cry proteins have a direct effect on AMF is equivocal. For example, lower AMF colonization levels have been reported in Bt maize lines Bt 11 (Castaldini et al., 2005; Cheeke et al., 2011) and Bt 176 (Turrini et al., 2004; Castaldini et al., 2005) expressing Cry1 Ab, but Bt maize (MON810) expressing the same Cryl Ab protein did not have lower AMF colonization when compared to its non-Bt parental isoline (de Vaufleury et al., 2007). There were also no negative effects on AMF reported for $B t$ cotton expressing Cry1Ac and Cry2Ab (Knox et al., 2008). However, AMF colonization was significantly lower in Medicago sativa grown for four months in soil amended with Bt 11 maize compared with $M$. sativa grown in soil amended with non-Bt maize (Castaldini et al., 2005). Because these studies were conducted under different experimental conditions with variations in AMF inocula, Bt cultivar, Cry protein, fertilizer level, harvest time, and assessment method, it has been difficult to compare results across studies. Moreover, the reduction in AMF colonization observed in certain $B t$ maize lines may also be due to indirect effects of the gene insertion, which may cause a change in root exudates or biochemical composition of the plant tissue, rather than to a direct effect of Cry protein on soil fungi (e.g., Naef, Zesiger, and Defago, 2006; Devare, Londono-R, and Thies, 2007). Given the initial indication that some lines of Bt maize are poorly colonized by AMF (Turrini et al., 2004; Castaldini et al., 2005; Cheeke et al., 2011), and that results to date have been inconsistent across studies, it is important to determine whether $B t$ maize lines expressing different numbers and types of engineered 
traits have a negative effect on arbuscular mycorrhizal fungi when evaluated under the same experimental conditions.

In this greenhouse study we addressed three specific questions: 1) Will a difference in AMF colonization be detected between different $B t$ and non- $B t$ maize lines grown under the same experimental conditions?; 2) If so, are these differences related to the expression of a particular $B t$ protein?; and 3) Does $B t$ maize cultivation have a negative effect on AMF colonization of a subsequently planted crop? To address the first two questions, we examined AMF colonization in nine $B t$ maize lines, differing in number and type of engineered trait, and five corresponding non- $B t$ near isogenic parental $(\mathrm{P})$ base hybrids (Table 13) at two different time points in the maize lifecycle. To investigate whether $B t$ crop cultivation has a negative impact on AMF colonization of a subsequently planted species, Glycine max (vegetable soybean; Sayamusume) was grown to maturity in soil that had been pre-conditioned for $60 \mathrm{~d}$ with $B t$ or non- $B t$ maize. We hypothesized that AMF colonization would be lower in the Bt maize lines (Turrini et al., 2004; Castaldini et al., 2005; Cheeke et al., 2011), and that AMF colonization would also be reduced in $G$. max grown in soil pre-conditioned with $B t$ maize (Castaldini et al., 2005). The consistent experimental conditions used in this study were optimized to reflect low-input agricultural systems to allow for maximal AMF colonization (e.g., Cheeke et al., 2011), and locally-collected agricultural soil was used to evaluate how each $B t$ and non- $B t$ maize cultivar responds to a natural community of AMF in the soil. 


\section{Materials and Methods}

\section{Experimental overview}

In the first phase of this study, microcosms were constructed with a common soil community (50\% local agricultural soil, $25 \%$ sterile sand, and $25 \%$ sterile soil-less potting media) and cultured with one $B t$ or non-Bt maize host plant, with 10 replicates of each cultivar (one plant in 10 separate $4 \mathrm{~L}$ pots), for a total of 140 plants in the experiment. After establishing a vegetative history in each microcosm for 60 days, five replicates of each $B t$ and $\mathrm{P}$ maize line were destructively harvested, and roots were assessed for AMF colonization (McGonigle et al., 1990). G. max was then seeded into each pre-conditioned microcosm and destructively harvested at maturity to determine whether AMF colonization would be reduced in plants grown in soil pre-conditioned with $B t$ maize. The five remaining replicates of each maize line were harvested at day 100 to assess AMF colonization at a different physiological time point in the maize lifecycle (when plants had started to produce ears). Growth responses (height, leaf number, chlorophyll content, root biomass, shoot biomass, and ear number) were recorded to determine whether plants with higher levels of AMF colonization exhibited any growth or yield benefits as a result of the symbiosis.

\section{Plant cultivars}

Nine different lines of $B t$ maize (Zea mays) and five corresponding non-Bt parental 
base hybrids were obtained from three seed companies (Syngenta Seeds Inc., Boise, ID, Monsanto Company, St. Louis, MO, and an additional representative seed industry seed supplier). Before planting, the $B t$ maize lines were assigned numbers B1-B9 and their corresponding non-Bt parental base-hybrids were assigned numbers P1-P5. Note that some non- $B t$ isolines were the base-genetics for more than one $B t$ line; P1 was the base hybrid for B1, P2 was the base hybrid for $\mathrm{B} 2$ and $\mathrm{B} 5, \mathrm{P} 3$ was the base hybrid for the $\mathrm{B} 3$ and $\mathrm{B} 6, \mathrm{P} 4$ was the base hybrid for $\mathrm{B} 4$, and $\mathrm{P} 5$ was the base hybrid for $\mathrm{B} 7, \mathrm{~B} 8$, and $\mathrm{B} 9$. The $B t$ maize lines obtained for this study differed in type (sweet corn or field corn), the Bt protein expressed (Cry1Ab, Cry34/35Ab1, Cry1F + Cry34/35Ab1, Cry1F, Cry3Bb1, $\mathrm{Cry} 1 \mathrm{Ab}+\mathrm{Cry} 3 \mathrm{Bb} 1)$, the number and type of inserted traits (insect protection: European corn borer, corn root worm, Mexican corn worm, Western bean cutworm, Black cutworm, fall armyworm, among others; herbicide protection: Glufosinate and/or Glyphosate tolerance), and background genetics, representing a cross-section of the broad range of $B t$ maize lines commercially available (Table 13). The non-Bt parental maize seeds obtained from Monsanto Co. are the corresponding parental lines to the $B t$ lines and were described as non- $B t$ near isoline control hybrids; and the corresponding non- $B t$ maize seeds obtained from Syngenta and the other seed industry supplier were described as near isogenic parental base-hybrids or parental isolines. We are prohibited by our seed agreement from disclosing more information about the background genetics, gene expression, $B t$ protein concentration, parental isolines, or other details related to genetics 
of these plant lines (both genetically modified and parental). For simplicity, we will refer to all $B t$ maize plants in this study as $(B t)$ and the non- $B t$ maize plants as parentals $(\mathrm{P})$. The nongenetically modified G. max seeds used in the second phase of the experiment were obtained from Territorial Seed Company (Cottage Grove, OR, USA) and were chosen to represent the corn-soybean rotation commonly practiced in the USA. Test of soil nutrients and AMF spore composition

Soil was collected from a certified organic field plot (previously sown in mixed vegetables) in March 2008 at the Washington State University Research and Extension Center (Vancouver, WA, USA) and analyzed for nutrients (24 ppm nitrogen $\left(\mathrm{NO}_{3}-\mathrm{N}\right)$, 108 ppm phosphorus (Weak Bray), 474 ppm potassium), percent organic matter (4.5\%), soil texture (silt loam), and soil pH (6.1) by an independent laboratory (A\&L Western Agricultural Laboratories, Portland, OR, USA). Prior to planting, spores were extracted from a composite sample of the agricultural soil and identified morphologically at the International Culture Collection of Vesicular Arbuscular Mycorrhizal Fungi (Morgantown, WV, USA). In the agricultural soil, spores were identified that represented six putative AMF taxa: Gigaspora rosea or albida, Glomus intraradices, Glomus mosseae, Glomus claroideum, Paraglomus occultum, and an undescribed Acaulospora (Morton, 2008).

For this study, we chose to use endogenous AMF inoculum from whole soil rather than defined additions of AMF spores or single species cultures. Inoculations with single 
AMF species or a specific number of spores provide limited information about how a plant might respond to a community of AMF in a natural or agroecosystem and give little insight into the plant-fungal associations that are likely to be encountered in the field. The use of endogenous mycorrhizal inocula in whole soil is more ecologically relevant than using defined additions of AMF spores or single species AMF cultures, and is more useful for predicting how different lines of $B t$ maize might respond to a natural community of AMF under field conditions. For effects of single species cultures on AMF colonization in Bt maize, see Cheeke et al. (2011), Castaldini et al. (2005), and Turrini et al. (2004).

\section{Construction of microcosms}

This experiment commenced in March 2008 in a research greenhouse at Portland State University (Portland, OR, USA). Seeds of each Bt and P maize cultivar were surface sterilized in a $10 \%$ bleach solution and planted into 4 L nursery pots containing a hand-mixed potting mix of 50\% non-sterile agricultural soil (Vancouver, WA, USA), $25 \%$ sterile sand, $25 \%$ sterile Sunshine Mix soil-less potting media (70-80\% Canadian sphagnum peat moss, perlite, dolomitic limestone, gypsum, wetting agent; Sun Gro Horticulture, Bellvue, Washington, USA), with the agricultural soil serving as the natural AMF inoculum. Ten replicates of each plant line were planted (one plant in 10 separate 4 L pots, representing 14 different $B t$ and $\mathrm{P}$ lines), for a total of 140 maize plants in the experiment. 
Growth conditions and fertilizer treatments

To account for microclimatic effects, pots were set up in a completely randomized design and rotated on the greenhouse bench each week using a randomization key. The daytime temperatures in the greenhouse were between $27^{\circ} \mathrm{C}$ and $32^{\circ} \mathrm{C}$ and nighttime temperatures were between $20^{\circ} \mathrm{C}$ and $27^{\circ} \mathrm{C}$, which reflect growing temperatures of many corn-growing regions in the USA. Photoperiod was from 6:00 to 20:00 every day, supplied via metal halide lights and natural sunlight. Humidity varied between 50 and 70 percent throughout the growing period. Plants were hand watered daily and fertilized every 2 weeks with $200 \mathrm{ml}$ of a dilute fertilizer $(0.23 \mathrm{~g} / \mathrm{L}$ of Peter's Professional All Purpose Plant Food 24-8-16, St. Louis, MO). Assessment of maize plant growth

Maize plant height and leaf number were recorded two weeks after planting, and at day 30,60, and 100. After root samples had been collected for AMF assessment, shoots and roots were separated and dried for at least 48 hours at $60^{\circ} \mathrm{C}$ for biomass data. Chlorophyll (Chl) content was collected from live leaves (Minolta SPAD-502 Leaf Chl meter) and the number of ears on each maize plant was recorded at day 100 . Test of Bt pre-conditioned soil on AMF colonization in G. max

After harvesting the 60 day maize plants, the soil microcosms were stored on a greenhouse bench for 30 days, mimicking the rest period between when one $B t$ crop is harvested and a different crop is planted. Glycine max was grown to maturity in five 
replicate pots containing soil that had been pre-exposed for 60 days with one $B t$ or non- $B t$ maize line. At harvest, data were collected on G. max height, root and shoot biomass (dry weight), bean pod number, and percent AMF colonization of roots. Mycorrhizal fungus colonization assessment

At harvest, roots were rinsed in tap water to remove soil particles and an equivalent amount of cut samples were taken from each root system. Roots were cleared using $10 \%$ $\mathrm{KOH}$, neutralized in $2 \%$ aqueous $\mathrm{HCl}$, and stained with $0.05 \% \mathrm{w} / \mathrm{v}$ trypan blue in lactoglycerol to visualize fungal structures (Phillips and Hayman, 1970) and at least 50 $\mathrm{cm}$ of roots from each plant were scored for mycorrhizal fungus colonization using the slide-intersect method (McGonigle et al., 1990). So that the researcher was not aware of which root type (Bt or non- $B t)$ was being analyzed at the time of data collection, histocassettes were mixed randomly, and slides were labeled when they were being prepared using a sequential number system that was not in any way associated with the $B t$ or P treatment. The presence/absence of hyphae, arbuscules, and vesicles observed per 100 root intersects was recorded for each sample. Total percentage AMF colonization was recorded as the total number of intersects out of 100 that had the presence/absence of any fungal structure (hyphae, arbuscules, and/or vesicles).

Data analysis

Differences in arbuscular mycorrhizal fungal colonization (hyphae, arbuscules, vesicles, and total percentage AMF colonization) and plant growth responses between $B t$ 
and P maize $(\alpha=0.05)$ were analyzed using the Proc Mixed procedure of SAS version 9.1 (SAS Institute, Cary, North Carolina, USA). The Proc GLM procedure of SAS version 9.1 was also performed for each analysis, but because the significant results were similar, we only included the Proc Mixed results here. To test for differences in AMF colonization between $B t$ and $\mathrm{P}$ maize, $B t$ was treated as a fixed effect and parental and $B t^{*}$ parental were treated as random effects. To test for differences in plant growth responses at 60 days (root biomass and shoot biomass) and 100 days (root biomass, shoot biomass, chlorophyll content of fresh leaves, and ear number per plant), $B t$, initial plant size (plant height x leaf no.), and AMF colonization levels were treated as fixed effects, and parental and $B t^{*}$ parental were treated as random effects. To test for differences in AMF colonization as affected by specific Cry protein, the influence of the parental lines were controlled for in the model by entering the average level of AMF colonization in the parental as a covariate, and each Cry protein was treated as a fixed effect for both the 60 and 100 day harvest. AMF data were arcsine square root transformed for each analysis, and maize root biomass was square root transformed for the 60 day analysis to meet the assumptions of the model.

The Proc Mixed procedure of SAS was used to test for differences in AMF colonization in G. max grown in soil pre-conditioned $B t$ or non-Bt maize. For the test of soil feedback on AMF colonization in G. max, the fixed effect was soil (soil pre-exposed for 60 days with a $B t$ or P maize cultivar). For the analysis of $G$. max growth responses 
(root biomass, shoot biomass, and bean pod number) in the pre-conditioned soil, the fixed effects were soil and AMF.

\section{Results}

Effect of maize cultivar on AMF colonization

At the 60 day harvest when plants were in a period of active growth, AMF colonization of roots was significantly lower in the $B t$ maize lines compared with the non-Bt parental maize plants $\left(\mathrm{F}_{1,4}=9.0, P=0.04\right.$; Fig. 6$)$. When analyzed by fungal structure, colonization by hyphae $\left(\mathrm{F}_{1,4}=5.63, P=0.08\right)$, arbuscules $\left(\mathrm{F}_{1,4}=6.46, P=\right.$ $0.06)$, and vesicles $\left(\mathrm{F}_{1,4}=1.03, P=0.37\right)$ were not statistically different between the $B t$ and non- $B t$ maize lines (Fig. 6). At the 100 day harvest when plants were starting to produce ears, percent colonization by arbuscules was significantly lower in the $B t$ maize lines $\left(\mathrm{F}_{1,4}=9.25, P=0.04\right)$ compared to the non-Bt parental lines (Fig. 7$)$. There was no significant difference in hyphal colonization $\left(\mathrm{F}_{1,4}=1.42, P=0.30\right)$, vesicles $\left(\mathrm{F}_{1,4}=0.02\right.$, $P=0.89)$, or total percent AMF colonization $\left(\mathrm{F}_{1,4}=3.39, P=0.14\right)$ detected between the $B t$ and non- $B t$ maize lines at the second harvest period when plants were near maturity (Fig. 7). Across all maize lines, percent AMF colonization was lower at the 100 day harvest when plants were producing ears than when they were in an active growth phase at the 60 day harvest (Figs. 6,7). 
Effect of AMF colonization and cultivar type on maize growth

At 60 days, percent AMF colonization was negatively correlated with shoot biomass (Pearson correlation coefficient $=-0.37, P=0.002 ;$ Proc mixed $\mathrm{F}_{1,58}=4.68, P=0.03$ ) but there was no effect of AMF colonization on root biomass $\left(\mathrm{F}_{1,57}=0.23, P=0.63\right)$. There was no difference in root biomass $\left(\mathrm{F}_{1,4}=0.72, P=0.44\right)$ or shoot biomass $\left(\mathrm{F}_{1,4}=\right.$ $0.27, P=0.63$ ) between the $B t$ and non- $B t$ maize cultivars at the 60 day harvest.

At the 100 day harvest, there was no effect of AMF colonization on root biomass $\left(\mathrm{F}_{1,58}=1.53, P=0.22\right)$, shoot biomass $\left(\mathrm{F}_{1,58}=3.83, P=0.06\right)$, or chlorophyll content of fresh leaves $\left(\mathrm{F}_{1,58}=0.13, P=0.72\right)$. However, maize plants with higher levels of AMF colonization had a lower ear number $\left(\mathrm{F}_{1,58}=3.88, P=0.05\right)$ at the 100 day harvest. There was no difference in shoot biomass $\left(\mathrm{F}_{1,4}=0.03, P=0.87\right)$, ear number $\left(\mathrm{F}_{1,4}=0.11, P=\right.$ $0.75)$, or chlorophyll content of fresh leaves $\left(\mathrm{F}_{1,4}=0.02, P=0.89\right)$ between the $B t$ and non-Bt maize cultivars, although the $B t$ maize plants had a significantly greater root biomass $\left(\mathrm{F}_{1,4}=9.19, P=0.04\right)$ than the non- $B t$ parental plants at the 100 day harvest. Initial plant size (height $x$ leaf number) was the best predictor of root biomass $\left(\mathrm{F}_{1,57}=\right.$ $\left.18.57, \mathrm{p}<0.0001 ; \mathrm{F}_{1,58}=18.10, \mathrm{p}<0.0001\right)$ and shoot biomass $\left(\mathrm{F}_{1,58}=50.42, \mathrm{p}<\right.$ $\left.0.0001 ; \mathrm{F}_{1,58}=10.62, P=0.002\right)$ at 60 and 100 days, respectively, for both $B t$ and $\mathrm{P}$ plants.

Effect of type of Cry protein expressed on AMF colonization in Bt maize

The type of Cry protein expressed in the different $B t$ maize lines was generally not a 
strong predictor of AMF infection among the $B t$ cultivars (Table 14). When controlled for the influence of the parental lines in the analysis, $B t$ maize lines expressing Cry $1 \mathrm{Ab}$ had higher AMF infection levels (hyphae, arbuscules, and total AMF) than other $B t$ lines at the 60 day harvest, but this was primarily driven by the high AMF colonization in the B9 cultivar (Fig. 6A, B, D). Bt maize lines expressing Cry1F had lower arbuscule colonization compared to the other Bt maize lines at 60 days (Table 14; Fig. 6B). At the 100 day harvest, $B t$ maize lines expressing Cry34/35Ab1 had higher AMF colonization levels (hyphae, arbuscules, vesicles, and total AMF) in roots compared with the other $B t$ maize lines (Table 14; Fig. 6). The best predictor of AMF infection in the different $B t$ lines at the 60 day harvest was the AMF infection level of the associated parental lines $\left(\mathrm{F}_{1,34}=11.30 ; P=0.002\right)$. There was no effect of parental line on AMF colonization detected at the 100 day harvest $\left(\mathrm{F}_{1,34}=0.00 ; P=0.99\right)$. Regardless of the specific type of Cry protein(s) expressed, Bt maize lines overall had lower AMF colonization than their non-Bt parental lines at the 60 day harvest (Fig. 6) and lower colonization by arbuscules at the 100 day harvest (Fig. 7).

Effect of soil pre-conditioned with Bt or P maize on AMF colonization, plant growth, and yield in vegetable soybean

When G. max was grown to maturity in soil pre-conditioned for 60 days with a $B t$ or non- $B t$ maize plant, there was no effect of the $B t$ pre-conditioned soil on arbuscular mycorrhizal colonization of $G$. $\max$ roots $\left(\mathrm{F}_{1,4}=0.18, P=0.69\right)$ nor was there an effect 
of the pre-conditioned soil on $G$. max root biomass $\left(\mathrm{F}_{1,4}=0.33, P=0.59\right)$, shoot biomass $\left(\mathrm{F}_{1,4}=0.40, P=0.56\right)$, or bean pod number at harvest $\left(\mathrm{F}_{1,4}=0.47, P=0.53\right)$.

\section{Discussion}

Genetically-modified $B t$ maize and the non-Bt parental lines differed in their level of mycorrhizal colonization in roots when grown in field-collected soil containing a natural community of AMF. When maize plants were in a period of active growth, total AMF colonization was significantly lower in the $B t$ maize lines compared to the non- $B t$ parental lines. When the maize plants were closer to maturity and starting to produce ears, arbuscule formation was lower in the Bt maize cultivars. Although there was some variation in mycorrhizal infection levels within the different $B t$ maize and non- $B t$ parental lines, the $B t$ maize cultivars collectively exhibited lower AMF colonization compared to the parental lines, regardless of the number or type of engineered trait, their genetic background, or the type of Cry protein(s) expressed. Moreover, as there was no difference in AMF colonization of G. max grown in the $B t$ or non-Bt maize preconditioned soil, this study supports other research indicating that reductions in AMF colonization are likely not a result of a direct toxic effect of $B t$ proteins (Donegan et al., 1995; Koskella and Stotzky, 2002; Ferreira et al., 2003), but may be a result of other factors, such as an indirect effect of the genetic insertion within each $B t$ plant line (e.g., Donegan et al., 1995; Flores, Saxena, and Stotzky, 2005; Naef, Zesiger, and Defago, 
2006) that may affect their ability to respond to or recruit AMF in the rhizosphere, or as a result of differences in the background germplasm of the parental line which may influence how derived lines interact with AMF and/or acquire nutrients in the soil.

Variations in AMF colonization levels have been reported in other crop varieties (e.g., maize, wheat) (Hetrick, Wilson, and Cox, 1992; Kaeppler et al., 2000; Sawers, Gutjahr, and Paszkowski, 2008), including commercial maize lines that were selected under conditions of high phosphorus fertilization (Kaeppler et al., 2000), but it is not clear why the $B t$ maize lines in this study had lower levels of AMF in their roots than the non- $B t$ controls at two different harvest periods. The genetic basis of mycorrhizal responsiveness has been documented in a variety of agricultural crop species including rice (Gao et al., 2007), wheat (Hetrick, Wilson, and Cox, 1992), and maize (Kaeppler et al., 2000), as well as in wild species such as big bluestem (Schultz et al., 2001) and St. John's Wort (Seifert, Bever, and Maron, 2009), so it is possible that the insertion of the $B t$ construct in different $B t$ maize lines could affect the plant-fungal symbiosis in some GM cultivars, although this is difficult to determine with the design of the present study. Pleiotropic effects (change in a single gene that affects multiple phenotypic traits) of a genetic insertion are not uncommon (e.g., Sheveleva et al., 1998; reviewed in Wang, Vinocur, and Altman, 2003) and certain types of genetic changes, such as those that influence physiology (i.e. sugar allocation, enzyme activity in roots, lignin content, etc.) may affect the ability of some $B t$ maize lines to form relationships with AMF. 
Alternatively, AMF colonization levels in the $B t$ maize roots may also be strongly influenced by the background genetics of the parental line. At the 60 day harvest, for example, the best predictor of AMF infection in the $B t$ lines was the infection level of the associated parental line. However, this does not explain why AMF colonization was lower in the $B t$ cultivars compared with the non- $B t$ parental maize lines when grown under the same conditions. Given that there is likely still a certain amount of variation between each $B t$ line and its near isogenic parental base-hybrid, more work should be conducted to explore possible mechanisms that may contribute to the lower levels of AMF colonization observed in multiple $B t$ maize lines.

We did not observe growth benefits for maize plants that had higher levels of AMF colonization in their roots at either 60 or 100 days. In fact, maize plants that had higher AMF colonization had reduced shoot biomass at 60 days and a lower ear number at 100 days. A negative effect of AMF on maize biomass has also been observed in other studies; maize plants grown in high phosphorus treatments with AMF had $88 \%$ of the above ground biomass of maize plants grown at high phosphorus treatments without $\mathrm{AMF}$, indicating that the AMF symbiosis can reduce plant biomass under certain growth conditions (Kaeppler et al., 2000). It is well known that the plant-AMF symbiosis is dynamic and can range from parasitism to mutualism depending on the growth stage of the plant, ecological conditions, differences in cultivation practices, and many other biotic and abiotic factors (Johnson, Graham, and Smith, 1997; Kiers, West, and Denison, 
2002; Hirsch, 2004; Jones and Smith, 2004). Because we grew these plants in a fixedvolume of soil under low-fertilizer conditions in the greenhouse, it is not known how the $B t$ and non- $B t$ maize lines in our study would respond to AMF in the field. However, it has been shown that even when no plant growth responses are detected, AMF can dominate the phosphate supply to the plant (Smith, Smith, and Jakobsen, 2003, 2004), thereby benefiting the host plant without observable growth differences at the time of harvest. It has also been demonstrated that colonization ability can vary among AMF taxa (e.g., Douds et al., 1998; Graham and Abbott, 2000; Burleigh, Cavagnaro, and Jakobsen, 2002). When roots are colonized by more than one species of AMF, plants can uptake more phosphorus and exhibit greater plant growth than when colonized by a single AMF species (e.g., Jansa, Smith, and Smith, 2008). Although we detected lower levels of AMF colonization in the $B t$ maize roots, we do not know if the $B t$ maize plants also had lower diversity of AMF taxa colonizing their roots. The local agricultural soil used in our study to inoculate the microcosms contained at least six different AMF taxa (Morton, 2008), so it is possible that, over time, one or a few more aggressive AMF species colonized the $B t$ roots (Graham and Abbott, 2000). More research, including molecular identification of the AMF taxa colonizing $B t$ and non- $B t$ maize roots, would help to determine whether $B t$ maize plants with lower levels of AMF colonization also have reduced diversity of AMF in their roots.

Historically, predictions of how different $B t$ plants may respond to AMF have been 
challenging because of the inconsistent results reported to date, even among $B t$ cultivars expressing the same protein. Complex interactions among soil organisms and the multitude of biotic and abiotic factors that contribute to mycorrhizal symbiosis in a given soil ecosystem have also been confounding factors in understanding the relationship between $B t$ plants and AMF. The complexity of the potential interactions of multiple types of $B t$ and non- $B t$ maize (e.g., herbicide-tolerance genes and gene products), on the responses of different maize lines to AMF infection were considered, however, previous studies have demonstrated little or no direct effect of the expression of herbicidetolerance genes on soil microbes, AMF, or other soil fauna (e.g., Siciliano and Germida, 1999; Dunfield and Germida, 2003; Kowalchuk et al., 2003; Dunfield and Germida, 2004; Krogh et al., 2007; Griffiths et al., 2008; reviewed in Lundgren et al., 2009). Moreover, in our study, the parental control isolines that expressed herbicide-tolerance genes had relatively high levels of AMF colonization in their roots, further indicating no direct effect of the expression on herbicide-tolerance genes on arbuscular mycorrhizae. Despite that we used only 10 replicates, and despite the variance that might influence AMF colonization in the different maize lines, our results demonstrated that AMF colonization was significantly lower in the $B t$ cultivars at both sampling dates. Many of the differences in colonization that were not significant may have been significant with a higher number of replicates, but this remains to be tested.

Mycorrhizal colonization has also been shown to vary within the same $B t$ maize line 
depending on fungal inoculum (species of AMF, mixed versus pure cultures), the growth stage of the plant (early development, active growth, or reproductive stage), spore density, and fertilizer treatment (Cheeke et al., 2011). Because previous studies have evaluated AMF colonization in only one $B t$ plant line and under different experimental conditions, it has been difficult to compare the results among studies. Thus, maintaining the same environmental conditions throughout an experiment is critical for detecting the effects of different $B t$ maize cultivars on mycorrhizal fungi. To our knowledge, this study is the first demonstration of a reduction in AMF colonization across multiple $B t$ maize lines grown under the same experimental conditions. The use of endogenous mycorrhizae in whole soil inocula allowed each $B t$ and non- $B t$ maize line to interact with a community of soil organisms that might be expected under field conditions, making this study more ecologically relevant than other greenhouse studies where only pure spore cultures of one AMF taxa were used (e.g., Turrini et al., 2004; Castaldini et al., 2005; Cheeke et al., 2011). Future experiments should be conducted at the field level to verify the ecological significance of these findings and to examine whether long-term Bt crop cultivation has a negative effect on the abundance or diversity of AMF propagules in the soil ecosystem over time. 


\section{Chapter 4: Field evaluation of arbuscular mycorrhizal fungal colonization in Bacillus thuringiensis toxin-expressing $(B t)$ and non-Bt maize}

Published as: Cheeke, T.E., Cruzan, M.B., and Todd N. Rosenstiel. 2013. A field evaluation of arbuscular mycorrhizal fungal colonization in $B t$ and non-Bt maize. Applied and Environmental Microbiology. 79(13): 4078-4086.

\section{Abstract}

The cultivation of genetically engineered Bacillus thuringiensis toxin-expressing (Bt) maize continues to increase worldwide, yet the effects $B t$ crops on arbuscular mycorrhizal fungi (AMF) in soil are poorly understood. In this field experiment, we investigated the impact of seven different genotypes of $B t$ maize and five corresponding non- $B t$ parental cultivars on AMF and evaluated plant growth responses at three different physiological time points. Plants were harvested 60 days (active growth), 90 days (tasseling and starting to produce ears), and 130 days (maturity) after sowing and data were collected on plant growth responses and percent AMF colonization of roots at each harvest. Spore abundance and diversity were also evaluated at the beginning and end of the field season to determine whether the cultivation of $B t$ maize had a negative effect on AMF propagules in the soil. Plant growth and AMF colonization did not differ between $B t$ and non- $B t$ maize at any harvest period, but AMF colonization was positively correlated with leaf chlorophyll content at the 130 day harvest. Cultivation of $B t$ maize had no effect on spore abundance and diversity in $B t$ versus non- $B t$ plots over one field season. Plot had the most significant effect on total spore counts, indicating spatial heterogeneity in the 
field. Although previous greenhouse studies demonstrated that AMF colonization was lower in some $B t$ maize lines, our field study did not yield the same results, suggesting that the cultivation of $B t$ maize may not have an impact on AMF in the soil ecosystem under field conditions.

\section{Introduction}

Genetically modified (GM) crops were commercially introduced in 1996 and now represent the majority of maize, cotton, and soybean grown in the USA (1). In 2012, $88 \%$ of the maize cultivated in the United States was genetically engineered to express herbicide tolerance, insect resistance, or some combination of stacked traits (1). Genetically modified crops also continue to be adopted by an increasing number of farmers worldwide (2). One of the most broadly cultivated GM crops is maize that has been genetically engineered to express one or more insecticidal toxins derived from the soil bacterium Bacillus thuringiensis (i.e. Bt corn). There are at least 60 different $B t$ crystalline (Cry) proteins that have been identified that are targeted to certain insect groups (reviewed in 3, 4). The $B t$ insecticidal toxins incorporated into crop plants help to protect against damage by agricultural pests such as the European corn borer (Ostrinia nubilalis) and corn root worm (Diabrotica virgifera). When an insect ingests Bt plant material, $B t$ proteins bind to specific receptors in the gut, killing the insect larvae (5, reviewed in 6). $B t$ toxins can enter soil through pollen deposition, incorporation of $B t$ 
crop residue through plowing, or through root exudates (reviewed in 3, 7). Genetic alterations within $B t$ plants may have non-target effects on soil organisms associated with plant roots, such as arbuscular mycorrhizal fungi (AMF). Despite the widespread cultivation of $B t$ crops, few studies have examined the interactions between $B t$ maize and symbiotic fungi in the soil ecosystem (reviewed in 3,7 ).

Arbuscular mycorrhizal fungi form symbiotic relationships with plant roots and have been shown to improve plant growth, enhance nutrient and water uptake, help protect against plant pathogens, and contribute to soil structure and function (8). Arbuscular mycorrhizal fungi are obligate symbionts, and thus require a plant host for nutrition and reproduction. Plants supply carbon to the fungi, and fungi provide the plant with nutrients such as nitrogen and phosphorus, and can improve drought tolerance (8). Recent studies have suggested that some types of $B t$ crops may have a negative impact on AMF (9-12), although the mechanism is not yet known. Although there is no evidence for a direct effect of $B t$ proteins on soil fungi, AMF may be uniquely sensitive to genetic changes within a plant because of their reliance on a host plant. In particular, AMF may be sensitive to alterations in root exudates $(13,14)$, differences in root architecture or physiology (e.g., 15, 16), or to changes in root enzymes (17-19) that may influence carbon dynamics in the rhizosphere (20-22).

Recent greenhouse studies demonstrated that AMF associations were reduced in multiple lines of $B t$ maize (9-12) and that differences in AMF colonization between $B t$ 
and non- $B t$ maize can vary as a result of experimental and environmental conditions, such as spore density and fertilizer level (10). Under low-fertilizer conditions, AMF associations with $B t$ maize were significantly lower than the non- $B t$ parental $(\mathrm{P})$ maize $(10,11)$. When residual effects of the cultivation of $B t$ maize were tested on a subsequently planted crop (Glycine max; soybean), there was no difference in AMF colonization of G. max grown to maturity in $B t$ or non-Bt pre-conditioned soil (11). However, lower AMF colonization was reported in Medicago sativa (alfalfa) grown in pots that had previously been cultivated in $B t$ maize and had $B t$ plant material incorporated into the soil (9). Other studies have reported no effect of $B t$ crop cultivation on AMF in greenhouse and microcosm studies (Bt maize: $23,24,25)$ or in field experiments (Bt cotton: 26). Because these studies were conducted on different types of $B t$ crops, and vary substantially in nutrient levels, spore density, growing conditions, plant age at harvest, and plant genotype, experimental results to date are difficult to compare. To date, there have been no studies that have evaluated the effects of the cultivation of $B t$ maize on AMF in the field. Given that several greenhouse studies, from independent research labs, have reported a negative effect of $B t$ maize on AMF, it is important to examine these symbiotic relationships under more natural field conditions.

In this field study, we evaluated AMF colonization and growth response of seven different lines of genetically modified $B t$ maize and five corresponding non- $B t$ parental isolines. Soil samples were collected from each plot at the beginning and end of the field 
season to determine whether spore abundance or diversity was reduced in the $B t$ plots after one growing season. Maize plants were harvested at three different physiological time points $(60,90$, and 130 days after sowing) to examine temporal differences in AMF colonization in each line of $B t$ and non- $B t$ maize and to evaluate potential differences in yield at the end of the season. Because we used the same $B t$ and non-Bt maize genotypes as in previous studies, we hypothesized that results from this field experiment would support our greenhouse studies $(10,11)$ and demonstrate that AMF colonization is lower in the $B t$ maize lines compared with their non-Bt parental controls under field conditions. While we acknowledge that there are differences in soil properties and likely differences in AMF communities between our greenhouse and field study, previous greenhouse studies, conducted in independent laboratories with different soils and different sources of AMF inocula (e.g. field soil, pure spores of Glomus mosseae), demonstrated an altered relationship between $B t$ maize and AMF (9-12), providing evidence that AMF colonization can be reduced in $B t$ maize under at least some environmental conditions. We also predicted that if AMF colonization levels were lower in the $B t$ maize lines, AMF spore abundance and diversity would also be lower in the $B t$ plots at the end of the field season. Finally, we hypothesized that plants with higher levels of AMF colonization in roots would have a greater shoot biomass and higher leaf chlorophyll content, consistent with a beneficial gain from the symbiosis. 


\section{Materials and Methods}

Study site

This field experiment was conducted from May to November 2009 near Corvallis, Oregon, USA, which is located in the Willamette Valley of Western Oregon. The climate in this region is relatively mild throughout the year and is characterized by cool, wet winters and warm, dry summers. The mean annual high temperature is $17.4^{\circ} \mathrm{C}$ and mean annual low temperature is $5.6^{\circ} \mathrm{C}$; the mean annual precipitation is $111 \mathrm{~cm} /$ year (26). The soil in this region is classified as Chehalis series fine-silty, mixed superactive, mesic Cumulic Ultic Haploxerolls (27). The soil at the field site has a clay loam texture $(22 \%$ sand, $50 \%$ silt, $27 \%$ clay), $\mathrm{pH}$ 5.7-6.1, medium levels of nitrogen (13-20 ppm $\left.\mathrm{NO}_{3}-\mathrm{N}\right)$ and potassium (333-438 ppm), and high levels of available phosphorus (27-32 ppm Weak Bray) (A\& L Western Agricultural Laboratories, Portland, Oregon, USA). The field site was previously a cow pasture with mixed grasses and forbs.

\section{Maize cultivars}

Seven different lines of $B t$ maize (Zea mays) and five corresponding non-Bt parental base hybrids were obtained from three seed companies (Syngenta Seeds Inc., Boise, ID, Monsanto Company, St. Louis, MO, and an additional representative seed industry seed supplier). The $B t$ maize lines (B1-B4, B6-B8) used in this study differed in type (sweet corn or field corn), the $B t$ protein expressed (Cry1Ab, Cry34/35Ab1, Cry1F + Cry34/35Ab1, Cry1F, Cry3Bb1), and background genetics (P1-P5), representing a cross- 
section of the broad range of $B t$ maize lines commercially available (7). The non- $B t$ maize seeds obtained from Monsanto Co. were described as non-Bt near isoline control hybrids, and the non-Bt maize seeds obtained from Syngenta and the other seed industry supplier were described as near isogenic parental base-hybrids or parental (P) isolines.

\section{Construction of plots}

The field site measured $35 \mathrm{~m} \mathrm{x} 10 \mathrm{~m}$ and had 24 plots, arranged in three sets of eight plots. Plots were $3 \mathrm{~m}$ long by $2 \mathrm{~m}$ wide, with a 1 meter buffer between plots and a $2 \mathrm{~m}$ buffer around the perimeter of the field site. On 26 May 2009, seeds of seven different $B t$ lines (B1-B4, B6-B8) and five corresponding non-Bt parental isolines (P1-P5; Table 13) were sown in replicate plots (each plot contained a single genotype), with 35-50 seeds per row, depending on previously determined germination rate of each cultivar. Each plot contained three rows, with $61 \mathrm{~cm}$ spacing between rows. Two replicate plots of each genotype were distributed randomly throughout the field site, representing 12 different $B t$ and non- $B t$ maize genotypes. After germination, plants were thinned to a maximum of 35 plants per row and each plant was given a unique identification number. No fertilizer was added to the field plots during this experiment and weeds were controlled by handpulling. Plants were irrigated with overhead sprinklers as necessary to ensure that plants were not drought stressed.

Test of AMF spore composition

Five soil samples were collected from the $0-15 \mathrm{~cm}$ fraction of soil along the center of 
each plot and pooled to determine the initial spore abundance and diversity in each plot prior to planting. Spores were extracted (28) and enumerated using the methods of McKenney and Lindsey (1987). Briefly, $10 \mathrm{~g}$ of soil was agitated in a 5\% Alconox solution to break up soil particles and wet-sieved using $20 \mathrm{~cm}$ diameter 500, 250, and 38 $\mu \mathrm{m}$ mesh sieves (28). Spores collected from the 38 and $250 \mu \mathrm{m}$ fraction were combined and centrifuged in a sucrose gradient (29). Quantification was carried out on Millipore membrane filters ( $47 \mathrm{~mm}$ diameter, $0.45 \mu \mathrm{m}$ pore size, with $3.1 \mathrm{~mm}$ square grids; Millipore Corporation, Billerica, MA, USA) after vacuum filtration (30). Spores were counted on filter paper using a stereomicroscope (Leica MZ16) and assigned to five different morphological categories based on colour and size (large black, large brown, medium brown, medium red, and small brown). At the end of the growing season, after plants had senesced, five soil samples were collected from the $0-15 \mathrm{~cm}$ fraction along the centre of each plot as processed as before to determine whether the plots that had been cultivated in $B t$ maize had a negative effect on AMF spore abundance or diversity after one growing season. Spores per gram soil were calculated based on soil dry weight (separate $10 \mathrm{~g}$ sample dried at $60^{\circ} \mathrm{C}$ for at least $48 \mathrm{hrs}$ and weighed).

\section{Assessment of maize plant growth}

Plants were harvested at 60,90 , and $130 \mathrm{~d}$ after sowing when plants were in an active growth stage, tasseling, and at maturity, respectively. Plant height and leaf number were recorded $45 \mathrm{~d}$ after sowing, and at each harvest to determine whether plants with higher 
levels of AMF colonization exhibited any growth benefits as a result of the symbiosis. Plant height was recorded from the base of each plant to the top of the tallest, outstretched leaf; leaf number was recorded as the total number of live and dead leaves on each plant (note: only live leaf number was used in the analysis); and leaf chlorophyll content was taken from the $5^{\text {th }}$ live leaf from the bottom of the plant using a chlorophyll meter (Minolta SPAD-502 Leaf Chl meter, Osaka, Japan). At each harvest, roots were sub-sampled for AMF assessment and then roots and shoots were dried at $60^{\circ} \mathrm{C}$ to constant weight. Once plants reached the reproductive stage (90 and $130 \mathrm{~d}$ after sowing), data were also collected on ear number per plant and weight of corn ears (dried in paper bags at $60^{\circ} \mathrm{C}$ to constant weight). Five plants were harvested from each plot $60 \mathrm{~d}, 10$ plants were harvested from each plot $90 \mathrm{~d}$, and 5 plants were harvested from each plot $130 \mathrm{~d}$ after sowing, for a total of 480 plants sampled over the course of the growing season. Based on preliminary studies, we anticipated the highest levels of AMF colonization at $90 \mathrm{~d}$ and reduced sampling load to 5 plants per plot at the 60 and $130 \mathrm{~d}$ harvests.

\section{Mycorrhizal fungus colonization assessment}

Roots were rinsed in tap water and subsamples of at least $50 \mathrm{~cm}$ were collected from each plant. Root samples were stained with a Trypan Blue solution to visualize fungal structures (31) and scored for mycorrhizal fungus colonization using the slide-intersect method (32). To ensure that the researcher was not aware of which root type (Bt or non- 
$B t$ ) was being analyzed at the time of data collection, histocassettes were mixed haphazardly during processing and slides were labelled using a sequential number system that was not associated with the $B t$ or P treatment.

\section{Data analysis}

Differences in initial spore abundance and diversity between plots $(\alpha=0.05)$ were analyzed using univariate ANOVA using the Proc GLM procedure of SAS (version 9.2). The Shannon Weaver Diversity Index $(\mathrm{H})$ was calculated as $\mathrm{H}=-\sum$ pi $\ln (\mathrm{pi})$ where pi is the relative abundance of each spore group. To test for differences in initial spore abundance and diversity between plots cultivated in Bt and P maize, "plant type" (Bt or non- $B t$ ) was treated as a fixed effect in the model; response variables were the spore categories (medium brown, large brown, large black, small brown, medium red, total spore number, and number of fungal taxa in one gram of dry soil). To test for differences in initial spore abundance and diversity between plots cultivated with each genotype of $B t$ or non-Bt maize, "cultivar" was treated as a fixed effect in the model with the same response variables as before. However, because there were only two replicate plots of each cultivar (due to limitations in field space and personnel), the primary emphasis for this data analysis is based on plant type (Bt vs. P). To test for differences in initial and final spore abundance as affected by variation in the field plots, "plot" was treated as a fixed effect in the model with total spores as the response variable. Because of unequal variance between initial and final soil samples, a Welch t-test was used to test for overall 
differences in initial (May 2009) versus final (October 2009) spore counts in each plot.

Differences in arbuscular mycorrhizal fungal colonization (hyphae, arbuscules, vesicles, and total percent AMF colonization) and plant growth responses between $B t$ and P maize $(\alpha=0.05)$ were analyzed using the Proc Mixed procedure of SAS (version 9.2). To test for differences in AMF colonization between $B t$ and $\mathrm{P}$ maize, $B t$ was treated as a fixed effect, and parental, $B t^{*}$ parental, and plot*row were treated as random effects. To test for differences in plant growth responses at 60 days (root and shoot biomass), 90 days (root biomass, shoot biomass, and ear number per plant), and 130 days (root biomass, shoot biomass, ear number per plant, and ear dry weight), $B t$, initial plant size (plant height x leaf \#), leaf chlorophyll content, and AMF colonization levels were treated as fixed effects, and parental, $B t^{*}$ parental, and plot*row were treated as random effects. To test for differences in leaf chlorophyll content at each harvest period, $B t$, initial size, and AMF colonization levels were treated as fixed effects and random effects were as previously described.

For each analysis, data were examined for normal distribution using Shapiro-Wilks tests and for equal variance using equal variance tests. Data were transformed as necessary to meet the assumptions of each model. Data analysis was performed using R software (version 2.14.1) and SAS (version 9.2). 


\section{Results}

Effect of Bt maize on spore abundance and diversity

There was no difference in initial spore abundance between $B t$ and non- $B t$ designated plots at the beginning of the growing season $\left(\mathrm{F}_{1,23}=0.26, \mathrm{P}=0.62 ; \mathrm{Fig} .8\right)$. The mean initial spore counts in $1 \mathrm{~g}$ of dry soil collected from $B t$ and P plots were 15.42 and 16.05, respectively. The mean numbers of fungal taxa in initial samples, as determined by spore morphology, in $B t$ vs. P plots were 4.00 and 3.90, respectively. The number of fungal taxa was not different between $B t$ and non- $B t$ plots at the beginning of the field season $\left(\mathrm{F}_{1,23}=0.10, \mathrm{P}=0.75\right)$. There was no difference in Shannon Index of Diversity $(\mathrm{H})$ between spores extracted from $B t$ and non- $B t$ plots at the beginning of the field season (0.98 and 0.87 , respectively; $\left(\mathrm{F}_{1,23}=3.09, \mathrm{P}=0.09\right)$.

At the end of the field season, after plants had senesced, there was no difference in AMF spore abundance between $B t$ and non- $B t$ plots $\left(\mathrm{F}_{1,118}=1.41, \mathrm{P}=0.24\right.$; Fig. 8$)$. The mean spore counts in $1 \mathrm{~g}$ of dry soil collected from $B t$ and P plots at the end of the season were 15.75 and 16.75 , respectively. The mean number of fungal taxa in final soil samples as determined by spore morphology in $B t$ vs. P plots were 3.80 and 3.50, respectively, and did not differ between $B t$ and $\mathrm{P}$ plots $\left(\mathrm{F}_{1,118}=3.66, \mathrm{P}=0.06\right)$. There was no difference in final spore diversity $(\mathrm{H})$ between $B t$ and non- $B t$ plots at the end of the field season $\left(\mathrm{H}=0.99\right.$ and $\mathrm{H}=0.95$, respectively; $\left.\mathrm{F}_{1,118}=1.79, \mathrm{P}=0.18\right)$. There was also difference between spore abundances in field plots between the beginning and end of the 
field season. Overall, total spore counts varied most by plot at the end of the field season $\left(\mathrm{F}_{1,23}=2.82, \mathrm{P}=0.0002\right)$ but this was not related to $B t$ or $\mathrm{P}$ cultivation. Because there was no effect of plant type ( $B t$ or $\mathrm{P}$ ) on spore abundance or diversity, spores were not identified to species.

\section{Effect of Bt maize on AMF colonization}

There was no difference in colonization by AMF hyphae, arbuscules, vesicles or total percentage AMF colonization between $B t$ and non- $B t$ maize at the $60 \mathrm{~d}$ harvest when plants were actively growing, at the $90 \mathrm{~d}$ harvest when plants were tasseling and starting to produce ears, or at the $130 \mathrm{~d}$ harvest when plants were mature (Table 15; Fig. 9). Mean AMF colonization levels were $29.69 \%$ in $B t$ maize and $28.94 \%$ in non- $B t$ maize at the 60 $\mathrm{d}$ harvest, $32.6 \%$ in $B t$ maize and $28.8 \%$ in non- $B t$ maize at the $90 \mathrm{~d}$ harvest, and $44.9 \%$ in $B t$ maize and $42.7 \%$ in non- $B t$ maize at the $130 \mathrm{~d}$ harvest.

Effect of AMF colonization and cultivar on maize growth

At the $60 \mathrm{~d}$ harvest when plants were actively growing, there was no effect of AMF colonization on root biomass, shoot biomass, or chlorophyll content of leaves (Table 16). Initial size was positively correlated with root biomass $($ Pearson correlation coefficient $=$ 0.74, $\mathrm{P}<0.0001$; Proc mixed $\mathrm{F}_{1,51}=56.52, \mathrm{P}<0.0001$ ), shoot biomass (Pearson correlation coefficient $=0.83, \mathrm{P}<0.0001$; Proc mixed $\left.\mathrm{F}_{1,51}=124.18, \mathrm{P}<0.0001\right)$, and leaf chlorophyll content $($ Pearson correlation coefficient $=0.55, \mathrm{P}<0.0001$; Proc mixed $\left.\mathrm{F}_{1,52}=49.46, \mathrm{P}<0.0001\right)$. Chlorophyll content in leaves at the time of harvest was 
positively correlated with root biomass (Pearson correlation coefficient $=0.68, \mathrm{P}<$ 0.0001; Proc mixed $\mathrm{F}_{1,51}=34.58, \mathrm{P}<0.0001$ ) and shoot biomass (Pearson correlation coefficient $=0.71, \mathrm{P}<0.0001 ;$ Proc mixed $\mathrm{F}_{1,51}=47.87, \mathrm{P}<0.0001$ ). There was no difference in root biomass, shoot biomass, or chlorophyll content between the $B t$ and non- $B t$ maize cultivars at the $60 \mathrm{~d}$ harvest (Table 17). Mean root biomass was $3.19 \mathrm{~g}$ in $B t$ maize and $3.62 \mathrm{~g}$ in non- $B t$ maize; mean shoot biomass was $28.85 \mathrm{~g}$ in $B t$ maize and $28.57 \mathrm{~g}$ in non-Bt maize; and mean leaf chlorophyll content was 47.87 in $B t$ maize and 46.76 in non- $B t$ maize at the $60 \mathrm{~d}$ harvest.

At the $90 \mathrm{~d}$ harvest when maize plants were tasseling and starting to produce ears, there was no effect of percentage AMF colonization on root biomass, shoot biomass, chlorophyll content of leaves, or ear number (Table 16). Initial size was positively correlated with root biomass (Pearson correlation coefficient $=0.54, \mathrm{P}<0.0001$; Proc mixed $\mathrm{F}_{1,167}=37.92, \mathrm{P}<0.0001$ ), shoot biomass (Pearson correlation coefficient $=0.63$, $\mathrm{P}<0.0001$; Proc mixed $\mathrm{F}_{1,168}=99.57, \mathrm{P}<0.0001$ ), leaf chlorophyll content (Pearson correlation coefficient $=0.34, \mathrm{P}<0.0001 ;$ Proc mixed $\mathrm{F}_{1,169}=45.37, \mathrm{P}<0.0001$ ), and ear number per plant (Pearson correlation coefficient $=0.45, \mathrm{P}<0.0001$; Proc mixed $\mathrm{F}_{1,168}=$ 22.68, $\mathrm{P}<0.0001)$. Chlorophyll content was positively correlated with root biomass (Pearson correlation coefficient $=0.58, \mathrm{P}<0.0001 ;$ Proc mixed $\mathrm{F}_{1,167}=102.44, \mathrm{P}<$ 0.0001), shoot biomass (Pearson correlation coefficient $=0.61, \mathrm{P}<0.0001$; Proc mixed $\left.\mathrm{F}_{1,168}=93.04, \mathrm{P}<0.0001\right)$, and ear number per plant $($ Pearson correlation coefficient $=$ 
$0.46, \mathrm{P}<0.0001$; Proc mixed $\mathrm{F}_{1,168}=48.81, \mathrm{P}<0.0001$ ). There was no difference in root biomass, shoot biomass, leaf chlorophyll content, or ear number between the $B t$ and non$B t$ maize cultivars at the $90 \mathrm{~d}$ harvest (Table 17). Mean root biomass was $7.59 \mathrm{~g}$ in $\mathrm{Bt}$ maize and $6.95 \mathrm{~g}$ in non-Bt maize; mean shoot biomass was $93.88 \mathrm{~g}$ in $B t$ maize and $89.97 \mathrm{~g}$ in non- $B t$ maize; mean leaf chlorophyll content was 46.35 in $B t$ maize and 48.02 in non-Bt maize; and mean ear number was 1.41 in $B t$ maize and 1.29 in non-Bt maize at the $90 \mathrm{~d}$ harvest.

At the $130 \mathrm{~d}$ harvest when maize plants had reached maturity, there was no effect of percentage AMF colonization on root biomass, shoot biomass, ear number, or ear weight (Table 16), however AMF colonization was positively correlated with chlorophyll content (Pearson correlation coefficient $=0.22, \mathrm{P}=0.02$; Table 16; Fig. 10). Initial size was positively correlated with root biomass (Pearson correlation coefficient $=0.62, \mathrm{P}<$ 0.0001; Proc mixed $\mathrm{F}_{1,89}=51.73, \mathrm{P}<0.0001$ ), shoot biomass (Pearson correlation coefficient $=0.68, \mathrm{P}<0.0001 ;$ Proc mixed $\left.\mathrm{F}_{1,89}=90.73, \mathrm{P}<0.0001\right)$, leaf chlorophyll content $\left(\right.$ Pearson correlation coefficient $=0.26, \mathrm{P}=0.005 ;$ Proc mixed $\mathrm{F}_{1,90}=17.05, \mathrm{P}<$ 0.0001), ear number (Pearson correlation coefficient $=0.38, \mathrm{P}<0.0001$; Proc mixed $\mathrm{F}_{1,89}$ $=10.62, \mathrm{P}=0.002)$, and ear weight $($ Pearson correlation coefficient $=0.67, \mathrm{P}<0.0001$; Proc mixed $\left.\mathrm{F}_{1,88}=84.28, \mathrm{P}<0.0001\right)$. Chlorophyll content was positively correlated with root biomass (Pearson correlation coefficient $=0.35, \mathrm{P}<0.0001$; Proc mixed $\mathrm{F}_{1,89}=$ 16.31, $\mathrm{P}=0.0001$ ), shoot biomass (Pearson correlation coefficient $=0.44, \mathrm{P}<0.0001$; 
Proc mixed $\left.\mathrm{F}_{1,89}=28.08, \mathrm{P}<0.0001\right)$, ear number $($ Pearson correlation coefficient $=0.48$, $\mathrm{P}<0.0001$; Proc mixed $\mathrm{F}_{1,89}=24.76, \mathrm{P}<0.0001$ ), and ear weight (Pearson correlation coefficient $=0.57, \mathrm{P}<0.0001 ;$ Proc mixed $\left.\mathrm{F}_{1,88}=48.47, \mathrm{P}<0.0001\right)$. There was no difference in root biomass, shoot biomass, chlorophyll content, ear number, or ear weight between $B t$ and non- $B t$ maize at $130 \mathrm{~d}$ (Table 17). Mean root biomass was $8.01 \mathrm{~g}$ in $B t$ maize and $7.37 \mathrm{~g}$ in non- $B t$ maize; mean shoot biomass (shoots + ears) was $185.92 \mathrm{~g}$ in $B t$ maize and $164.16 \mathrm{~g}$ in non- $B t$ maize, mean leaf chlorophyll content was 40.58 in $B t$ maize and 42.54 in non- $B t$ maize, mean ear number was 1.47 in $B t$ maize and 1.38 in non- $B t$ maize, and mean ear weight was $116.04 \mathrm{~g}$ in $B t$ maize and $103.76 \mathrm{~g}$ in non- $B t$ maize at the $130 \mathrm{~d}$ harvest.

Arbuscular mycorrhizal fungal colonization was highest in the $130 \mathrm{~d}$ samples (Fig. 9) and total plant biomass increased with each harvest (Fig. 11). Variation in plot had the most significant effect on AMF colonization and growth responses throughout the experiment as assessed using proc GLM in SAS ( $60 \mathrm{~d}$ : root biomass $\mathrm{F}_{1,23}=1.63, \mathrm{P}=0.05$; 90 d: $A M F F_{1,23}=4.65, P<0.001$, root biomass $F_{1,23}=2.23, P=0.002$, leaf chlorophyll content $\mathrm{F}_{1,23}=2.38, \mathrm{P}=0.0006 ; 130 \mathrm{~d}: \mathrm{AMF}_{1,23}=4.92, \mathrm{P}<0.001$, root biomass $\mathrm{F}_{1,23}=$ 2.16, $\mathrm{P}=0.005$, shoot biomass $\mathrm{F}_{1,23}=2.36, \mathrm{P}=0.002$, leaf chlorophyll content $\mathrm{F}_{1,23}=3.44$, $\mathrm{P}<0.0001$; ear number $\mathrm{F}_{1,23}=1.86, \mathrm{P}=0.02$ and total ear weight $\mathrm{F}_{1,23}=2.28, \mathrm{P}=0.003$ ). 


\section{Discussion}

In this field study, there were no differences observed in AMF colonization between $B t$ and non- $B t$ maize 60 days, 90 days, or 130 days after sowing. Based on previous greenhouse studies, we predicted that field-grown $B t$ maize would display a lower level of AMF colonization compared to non-Bt maize at each harvest period, but this hypothesis was not supported. This is surprising because the same $B t$ maize genotypes that had previously exhibited lower AMF colonization in greenhouse studies (11) were also utilized here. Further, we detected no difference in plant biomass, leaf chlorophyll content, ear number, or ear weight between $B t$ and non-Bt maize at any harvest date. However, AMF was positively correlated with leaf chlorophyll content at the 130 day harvest when plants were fully mature. We found no difference in spore counts between soil collected from $B t$ versus $\mathrm{P}$ plots at the beginning or end of the field season, and our counts were similar to spore densities reported in other maize field studies (34-36). While our spore diversity was low compared to many natural systems, it is typical of the low mycorrhizal diversity reported for other agricultural and monocropping systems $(37,38)$. Although there was no difference in AMF spore abundance and diversity in field plots at the beginning of the field season, there was a significant effect of plot on total spore counts at the end of the field season, indicating spatial heterogeneity of AMF propagules in these field plots. However, these differences in spore counts between plots were not a result of maize genotype (Bt vs. P). 
The symbiosis between maize and AMF can vary strongly depending on experimental or environmental conditions (10), and more generally the plant-AMF relationship can fluctuate along a parasitism-mutualism continuum (39). Arbuscular mycorrhizal fungi are considered parasitic when the net cost of the symbiosis exceeds net benefits for the plant and are mutualistic when both partners benefit from the relationship, although there have been some recent discussions on the use of these terms $(40,41)$. Our field experiment showed increasing levels of AMF colonization in both $B t$ and non- $B t$ parental maize roots over time, with the highest levels of AMF colonization detected at the 130 day harvest when plants were mature. These results support the findings of Grigera et al. (2007a) who documented an increase in carbon allocation to AMF during the reproductive period of maize (42) and demonstrated that AMF were most abundant at the end of the maize growing season as assessed by fatty acid methyl esters (FAME) biomarkers (43). We also found a positive correlation between percent AMF colonization of roots and chlorophyll content of live leaves at the 130 day harvest, suggesting that the higher levels of AMF colonization led to higher nitrogen levels in maize at maturity (e.g., 44, 45, 46). While variation in soil nitrogen availability might have influenced mycorrhizal colonization levels (and thus affected leaf chlorophyll content), plots were randomly assigned to $B t$ and non- $B t$ cultivars prior to planting and data were combined in a single analysis where differences among plots were controlled for statistically. Thus, we were able to assess the overall relationship between percent AMF colonization maize roots and leaf chlorophyll 
content, minimizing any plot-specific nitrogen effects. The conditions of the field site may also help to explain the increase in AMF colonization in the maize roots at the end of the growing season. The study site was historically covered with mixed pasture grasses and forbs that were likely in symbiosis with AMF. When our study commenced, these plants were removed and the ground turned under. As weeds were hand-pulled throughout the study, the cultivated corn was the only host plant for the AMF in our field plots.

Variation in soil conditions may also be a key factor influencing the relationship between AMF and $B t$ and non-Bt maize (47). When nitrogen and phosphorus are readily available in soil, plants often have lower levels of AMF colonization in roots because the carbon cost of supporting fungal symbionts is higher than the benefits received (e.g., 10, 48). In previous greenhouse studies, we found that AMF colonization was lower in multiple lines of $B t$ maize grown in 50\% field soil collected from Vancouver, Washington, USA (11) and that $B t$ and non- $B t$ maize grown without fertilizer or in low fertilizer treatments $\left(0.23 \mathrm{~g} \mathrm{~L}^{-1}\right)$ recruited more AMF than maize grown in high fertilizer (1.87 $\mathrm{g} \mathrm{L}^{-1}$ ) treatments (10). However, in the current Corvallis field study we observed no differences in AMF colonization between many of the same lines of $B t$ and non- $B t$ maize used in the greenhouse study. Although we did not fertilize our field plots, the maize plants did not exhibit any obvious signs of nutrient stress and grew with vigour, indicating that the soils were not nutrient limited. The Corvallis field soils differ in 
nutrient availability and likely contain a different community of AMF than the Vancouver soils, potentially explaining the contradictory results we've observed. Future investigation of the differences in soil nutrient availability and spore composition on AMF colonization of $B t$ and non- $B t$ maize will help to elucidate the interplays between these plant-fungal partners. The significant plot effects observed in the growth responses and AMF colonization levels in our maize plants suggest spatial heterogeneity of nutrient availability and/or spore density in the soil, however, these were not related to maize genotype. Increasing the plot number of each cultivar in future field studies would likely help to minimize the impact of spatial heterogeneity in similar studies.

Interestingly, we detected no differences in spore abundance between field plots at the beginning and end of the growing season. There are several potential reasons for this. The field site was plowed prior to soil collection and planting in the spring, so perhaps the spores that were in the soil at that time were not actively colonizing the weeds/pasture plants at the time of plot preparation (i.e. spore bank). We collected final soil samples at the end of the field season after plants had senesced because we expected spore production to be the highest in the fall after plants had produced seed. It is possible, however, that we missed the sporulation event (perhaps it was in the late summer) and spores re-colonized any remaining maize roots or weeds that grew after the $130 \mathrm{~d}$ harvest. It is also possible that in this system, roots and vesicles were serving as propagules instead of spores. Indeed, one study that took place in vineyards in the Willamette Valley 
of Western Oregon reported a similar number of AMF species in roots and soil (based on amplification of AMF DNA in root samples and spore morphology), however, roots and soil had a different AMF community, indicating that the spores in the soil may not necessarily reflect the AMF taxa actively colonizing plant roots (49). This lends support to the idea that there may be a spore bank in our field soil that may not represent the AMF taxa colonizing the maize plants in our study, but this remains to be tested.

Future investigations evaluating the impact of $B t$ maize and other genetically modified agronomic species on AMF in the soil ecosystem will be beneficial to both the scientific and agricultural community. Although crop plants that are irrigated and fertilized may not benefit significantly from symbiosis with AMF (reviewed in 50), arbuscular mycorrhizal fungi are important for nutrient acquisition and drought tolerance in many sustainable agricultural and/or low input systems (reviewed in 51, 52, 53), and are important considerations in crop rotation $(54,55)$ and for native plant establishment in grassland restorations of former agricultural fields (e.g., 56, 57). Arbuscular mycorrhizal fungi can also be affected by tillage (e.g., 58, 59, 60), plant type (e.g., 54, 61), and management practices (e.g., 62, 63). Although results from our field experiment indicate no difference in spore abundance and diversity in the soil, and no differences in AMF colonization levels between $B t$ and non- $B t$ maize over one growing season, the diversity of AMF colonizing the various maize genotypes remains unknown. Future studies should aim to resolve the causal factors contributing to the widespread variation 
between AMF and Bt maize which has been observed to date and would benefit from determining whether there is any variation in taxonomic and/or functional diversity of AMF colonizing $B t$ maize and non- $B t$ parental isolines under field conditions. 


\section{Chapter 5: Effect of $\boldsymbol{B t}$ maize cultivation history on arbuscular mycorrhizal fungal colonization, spore abundance and diversity, and plant growth}

To be submitted as: Tanya E. Cheeke, Hayley Darby, Todd N. Rosenstiel, James D. Bever, and Mitchell B. Cruzan.

\section{Abstract}

Recent greenhouse studies have reported that maize expressing Bacillus thuringiensis $(B t)$ insecticidal toxins may have nontarget effects on symbiotic arbuscular mycorrhizal fungi (AMF), however, field studies have not detected the same pattern. This may be due to the short-term nature of previous field experiments, differences in soil properties between studies, or plant-soil feedbacks that influence AMF communities in roots and soil over time. In this field experiment, we used split plots to evaluate the effect of $B t$ or non- $B t$ maize cultivation history on growth of seven different genotypes of $B t$ maize and five corresponding non-Bt parental $(\mathrm{P})$ isolines, spore abundance and diversity in soil, and AMF colonization of roots. We found that $B t$ plants had higher leaf chlorophyll content when they were grown in plots that had been cultivated with $B t$ maize the previous year, and similarly, non-Bt plants had higher chlorophyll content when they were grown in plots with a non-Bt cultivation history. There was a greater density of spores in plots with a $\mathrm{P}$ cultivation history than in plots where $B t$ maize had been grown in the previous year, but no difference in spore diversity. In spite of the difference in spore density, we found no significant differences in AMF colonization or root or shoot 
biomass between plots with a cultivation history of $B t$ and P maize. Results of this study indicate that the symbiotic relationship between maize and AMF is dynamic and that differences in AMF colonization between cultivars may be influenced by propagule distribution in the field, plot history, soil conditions, and other biotic and abiotic factors.

\section{Introduction}

The relationship between genetically modified (GM) plants and arbuscular mycorrhizal fungi (AMF) is an important element of soil ecology research. AMF are ubiquitous in both natural and agroecosystems and form symbiotic relationships with most land plants (Wang and Qiu 2006, Smith and Read 2008). In the plant/AMF symbiosis, plants provide carbon to the fungi in the form of photosynthate and AMF provide nutrients (mainly $\mathrm{P}$ and $\mathrm{N}$ ) and water to the plant by increasing the surface area of plant roots (Smith and Read 2008). AMF are also important for improving soil aggregation through the production of glomalin and for protecting against root pathogens (Smith and Read 2008). While AMF are known to be sensitive to a variety of agricultural factors, including tillage (Douds et al. 1995, Galvez et al. 2001), pesticides (Trappe et al. 1984), and fertilizer applications (Johnson et al. 1991, Johnson et al. 2008), it is not well understood how AMF may be impacted by the cultivation of Bacillus thuringiensis (Bt) protein expressing crops over time, including $B t$ maize.

Bacillus thuringiensis maize is genetically engineered to express one or more insecticidal toxins derived from $B t$ soil bacteria to protect plants against damage by a 
variety of insect pests including Lepidopteran, Coleopteran, and Dipteran larvae (reviewed in Icoz and Stotzky 2008, Cheeke 2012). Globally, Bt maize is one of the most widely cultivated genetically modified crops, and in 2012 , GM varieties comprised $88 \%$ of all maize planted in the USA (USDA 2012). There are more than $60 \mathrm{Bt}$ proteins that are highly targeted to certain insect groups (reviewed in Icoz and Stotzky 2008, Sanchis 2011). Bt proteins work by binding to specific receptors in the guts of susceptible larvae, liquefying the gut and killing the insect (Federici 1993, reviewed in Bravo et al. 2007). While specific in their mode of action, $B t$ proteins can also enter soil and waterways through root exudates, decomposing plant material, and/or pollen deposition (reviewed in Icoz and Stotzky 2008, Cheeke 2012) where they can remain biologically active for at least several months (Tapp and Stotzky 1998, Zwahlen et al. 2003, Tank et al. 2010). Because of the widespread cultivation and rapid adoption of genetically modified $B t$ crops worldwide, questions have arisen about the short-term and long-term effects of transgenic crop cultivation on nontarget organisms in the soil ecosystem over time.

Although there are many benefits of $B t$ crops (e.g., reduced chemical insecticide use, less insect damage on plant, lower exposure to insecticides for agricultural workers), recent studies have reported a negative effect of some $B t$ plants on arbuscular mycorrhizal fungi (Turrini et al. 2004, Castaldini et al. 2005, Cheeke et al. 2011, Cheeke et al. 2012), nematodes (Hoss et al. 2008), and nontarget insect larvae (Dively et al. 2004, Rosi-Marshall et al. 2007). Other studies have reported no negative effect of $B t$ crop 
cultivation on AMF (de Vaufleury et al. 2007, Knox et al. 2008, Tan et al. 2011, Verbruggen et al. 2012, Cheeke et al. 2013) and other soil organisms (reviewed in Icoz and Stotzky 2008, Cheeke 2012). While there is no evidence of a direct effect of $B t$ proteins on $\mathrm{AMF}$, it is possible that genetic changes within a plant (either through genetic engineering or traditional approaches) can affect a plant's relationship with symbiotic organisms. If genetic changes within a plant resulted in an alteration of plant root exudates (Bais et al. 2006, Broeckling et al. 2008), enzyme activity (Schaarschmidt et al. 2007), or chemical signals (Akiyama et al. 2005), for example, AMF (and other soil organisms) may be affected. Because AMF are obligate symbionts that require a plant host for survival and obtain their carbon by living within root cells, they may be more sensitive to genetic changes within a plant than other soil organisms, even if they are not affected by $B t$ proteins directly.

Cropping history may contribute to feedbacks that can enhance or inhibit plantmicrobe relationships in agricultural systems (Johnson et al. 1991, Bullock 1992). In the Midwestern United States, for example, crop rotations are commonly employed to mitigate problems associated with monocultures such as nutrient depletion, pathogen buildup, and pest resistance (Bullock 1992, Kinkel et al. 2011). In natural systems, positive plant-soil feedbacks have been shown to reduce plant diversity while negative plant-soil feedbacks tend to increase plant diversity (Bever et al. 2012). Plant-soil feedbacks have also been shown to have both positive and negative effects on the AMF 
community (Bever 2002, Bainard et al. 2009). For example, plants that have a higher dependence on AMF may lead to higher AMF infection potential of the soil at the end of the field season than those that do not form AMF associations (e.g. members of the Brassicaceae) or have a negative effect on AMF (e.g. endophyte-infected tall fescue, invasive plants) (Stinson et al. 2006, Callaway et al. 2008, Mack and Rudgers 2008, Bainard et al. 2009). Thus, plants that have a reduced association with AMF, no association with $\mathrm{AMF}$, or a negative impact on $\mathrm{AMF}$ may reduce $\mathrm{AMF}$ propagules in the soil over time (Vogelsang and Bever 2009), potentially affecting AMF colonization of roots in a subsequently planted crop (Gavito and Miller 1998, discussed in Bever et al. 2012, Koide and Peoples 2012). Previous greenhouse studies have demonstrated that some lines of $B t$ maize have a negative impact on AMF in roots (Turrini et al. 2004, Castaldini et al. 2005, Cheeke et al. 2011, Cheeke et al. 2012), however, it is not known if AMF propagules in the soil will be reduced over time in field plots with a history of $B t$ maize cultivation.

Field plots were cultivated in a single genotype in 2009 (Cheeke et al. 2013) and in the following year, paired $B t /$ non- $B t$ maize lines were grown in split plots with either a $B t$ or non-Bt cultivation history. In this study, we addressed four specific questions: (1) Will AMF spore abundance and diversity be lower in plots with a $B t$ cultivation history compared to plots with a non-Bt cultivation history? (2) If so, will AMF colonization be correspondingly lower in maize grown in plots with a history of $B t$ maize cultivation or in 
$B t$ plants compared with non-Bt plants grown in the same plot? (3) Will maize plants have higher root biomass, shoot biomass, and/or leaf chlorophyll content in plots with a history of cultivation with self (Bt or non-Bt maize)? (4) Will plants with higher levels of AMF colonization have a greater root and shoot biomass or higher chlorophyll content in leaves as a result of the symbiosis? Based on our earlier greenhouse studies (Cheeke et al. 2011, Cheeke et al. 2012) that demonstrated reduced AMF colonization in the same lines of $B t$ maize tested here, we hypothesized that AMF propagules would be lower in plots with a history of $B t$ maize cultivation and that $\mathrm{AMF}$ colonization would be lower in $B t$ maize compared with their non-Bt parental isolines when grown in the same split-plots. We also hypothesized that plants with higher levels of AMF colonization would have higher leaf chlorophyll content and greater shoot biomass as a result of the symbiosis, and that $B t$ and non- $B t$ maize would have a more positive growth response when grown in plots previously cultivated with self than with non-self (i.e., positive feedback response).

\section{Materials and Methods}

Study site

This field experiment was conducted from May to September 2010 in Corvallis, Oregon, USA. The climate in the Willamette Valley of Western Oregon is characterized by cool, wet winters and warm, dry summers. The mean annual low temperature is $5.6^{\circ} \mathrm{C}$, 
mean annual high temperature is $17.4^{\circ} \mathrm{C}$, and mean annual precipitation is $111 \mathrm{~cm} /$ year (NOAA 2012). The soil at the field site has a clay loam texture (22\% sand, $50 \%$ silt, $27 \%$ clay), $\mathrm{pH}$ 5.7-6.1, medium levels of nitrogen (13-20 ppm $\left.\mathrm{NO}_{3}-\mathrm{N}\right)$ and potassium (333$438 \mathrm{ppm}$ ), and high levels of available phosphorus (27-32 ppm Weak Bray) (A\& L Western Agricultural Laboratories, Portland, Oregon, USA) and is classified as Chehalis series fine-silty, mixed superactive, mesic Cumulic Ultic Haploxerolls (Natural Resources Conservation Service 2012).

\section{Maize cultivars}

In this field study, we used seven different genotypes of $B t$ maize (Zea mays) that had exhibited reduced AMF colonization in previous greenhouse studies (Cheeke et al, 2012) and five corresponding non-Bt parental $(\mathrm{P})$ base hybrids, representing both sweet corn and field corn (Table 13). The Bt genotypes differed in the Bt protein expressed (Cry1Ab, Cry34/35Ab1, Cry1F + Cry34/35Ab1, Cry1F, Cry3Bb1) and background genetics. Seeds were obtained from three companies (Syngenta Seeds Inc., Boise, ID, Monsanto Company, St. Louis, MO, and an additional seed industry supplier that prefers to remain anonymous). The non- $B t$ maize seeds obtained from Monsanto Co. were described as non- $B t$ near isoline control hybrids, and the non- $B t$ maize seeds obtained from Syngenta and the other seed industry supplier were described as near isogenic parental base-hybrids or parental isolines. 


\section{Construction of plots}

The field site measured $35 \mathrm{~m}$ x $15 \mathrm{~m}$ and had 28 plots arranged randomly in four incomplete blocks. In 2009, 24 plots were cultivated with a single $B t$ or non- $B t$ genotype to establish a $B t$ or non- $B t$ history and data were collected on spore abundance and diversity, AMF colonization, and growth responses of each line of maize (Cheeke et al. 2013). In 2010, each plant genotype was matched with its $B t$ or non-Bt counterpart (Table 13) and grown in split-plots with either a $B t$ or non- $B t$ history. Four additional split-plots were added in 2010 to account for $B t$ genotypes that shared the same parental cultivar (Table 13). These additional plots were used for comparison of growth responses and percent AMF colonization of roots between $B t$ and non- $B t$ plants, but were not included in the cultivation history or spore density analyses. There were four replicate plots of each $B t / P$ combination, half with a $B t$ history and half with a P history. Split-plots were planted with two rows of 35 seeds each (one row of $B t$ and one row of its corresponding non- $B t$ parental cultivar). After germination, plants were thinned to a maximum of 25 plants per row and each plant was given a unique identification number. No fertilizer was added to the field plots and weeds were controlled by hand. Plants were irrigated with overhead sprinklers as necessary to ensure that plants were not water stressed.

\section{Test of AMF spore composition}

To examine the effect of $B t$ or non- $B t$ plot history on spore abundance and diversity, five replicate soil samples were collected from the $0-15 \mathrm{~cm}$ fraction of soil along the 
center of each plot on May 24, 2010 during field preparation. Spores were extracted from three soil samples from each plot (Gerdemann and Nicolson 1963) and enumerated using the methods of McKenney and Lindsey (1987), as described in Cheeke et al., (2013). Assessment of maize plant growth

Plants were harvested 60 days after sowing, when plants were in an active growth stage. Plant height, leaf number, and chlorophyll content of live leaves were recorded 30 days after sowing and again at 60 days, along with shoot biomass, root biomass, and percent AMF colonization in roots to determine whether plants with higher levels of AMF colonization had a greater growth response as a result of the symbiosis. Plant height was measured from the base of the plant to the tallest, outstretched leaf. Leaf number was recorded as the total number of live and dead leaves on the plant (note: only live leaf number was used in the analyses). Leaf chlorophyll content was recorded from the fifth live leaf from the base of the plant using a chlorophyll meter (Minolta SPAD-502 Leaf Chl meter, Osaka, Japan). At harvest, subsamples of roots were collected for AMF assessment and roots and shoots were dried at $60^{\circ} \mathrm{C}$ to a constant weight for biomass data. Twelve plants were harvested from each plot ( $6 B t$ and 6 non- $B t)$ for a total of 336 plants in the analysis.

Mycorrhizal fungus colonization assessment

Soil was rinsed from roots in tap water and at least $50 \mathrm{~cm}$ of roots were collected from each plant for AMF colonization assessment. A Trypan Blue solution was used to 
visualize fungal structures (Phillips and Hayman 1970) and roots were scored for AMF colonization using the slide-intersect method (McGonigle et al. 1990). Histocassettes were mixed randomly during processing and slides were labeled using a sequential number system so that the researcher was not aware of which plant type (Bt or non-Bt) was being analyzed at the time of analysis.

Data analysis

Differences in spore abundance and diversity between plots with a $B t$ or P history ( $\alpha$ $=0.05$ ) were analyzed using univariate ANOVA and MANOVA with the Proc GLM procedure of SAS (version 9.2, SAS Institute, Cary, North Carolina, USA). The Shannon Weaver Diversity Index $(\mathrm{H})$ was calculated as $\mathrm{H}=-\sum p_{i} \ln \left(p_{i}\right)$ where $p_{i}$ is the relative abundance of each spore group $(i)$. To test for differences in spore abundance and diversity between plots with a $B t$ or P plot history, plot was nested within history and treated as a random effect; response variables were the spore categories (medium brown, large brown, large black, small brown, medium red, total spore number, and number of taxa in one gram of dry soil).

Differences in arbuscular mycorrhizal fungal colonization (hyphae, arbuscules, vesicles, and total percent AMF colonization) and plant growth responses between $B t$ and P maize were analyzed using the Proc Mixed procedure of SAS (version 9.2). To test for overall differences in AMF colonization between $B t$ and $\mathrm{P}$ maize grown in split plots, $B t$ was treated as a fixed effect, and parental, $B t^{*}$ parental, and plot*row were treated as 
random effects. To test for overall differences in plant growth responses between $B t$ and P maize (root biomass, shoot biomass, and leaf chlorophyll content), $B t$, initial plant size (plant height $\mathrm{x}$ leaf \#), AMF colonization, and leaf chlorophyll content were treated as fixed effects, and parental, $B t^{*}$ parental, and plot*row were treated as random effects.

To test for effects of plot history on AMF colonization, initial leaf chlorophyll content, root biomass, shoot biomass, and final leaf chlorophyll content, fixed effects in the model were $B t$, history, and $B t$ *history, and random effects were parental and $B t^{*}$ history*plot*row. Within this analysis, the $B t^{*}$ history interaction corresponds to the pairwise feedback interaction coefficient (Bever et al. 1997). AMF data were arcsin square root transformed prior to analysis and growth response data were log transformed as necessary to meet the assumptions of each model.

\section{Results}

\section{Effect of plot history on spore abundance and diversity}

Plots that were cultivated with a parental maize genotype in 2009 had higher numbers of total spores $\left(\mathrm{F}_{1,22}=5.94, \mathrm{P}=0.02\right)$ at the beginning of the 2010 field season compared to plots with a $B t$ maize history (Fig. 12). The mean total number of spores in 1 gram of dry soil from plots with a $B t$ or P history was 15.57 and 19.27, respectively. However, there was no difference in abundance of individual spore morphotypes between plots with a $B t$ or $\mathrm{P}$ history (medium brown, $\mathrm{F}_{1,22}=2.73, \mathrm{P}=0.11$; large brown, $\mathrm{F}_{1,22}=0.06, \mathrm{P}=$ 
0.81; large black, $\mathrm{F}_{1,22}=2.38, \mathrm{P}=0.14$; small brown, $\mathrm{F}_{1,22}=3.93, \mathrm{P}=0.06$; or red spores $\left(F_{1,22}=0.02, P=0.89\right)$. There was no difference in the Shannon Index of Diversity between spores extracted from plots with a $B t$ or non- $B t$ history $(0.79$ and 0.83 , respectively; $\left.\mathrm{F}_{1,22}=0.52, \mathrm{P}=0.48\right)$ and there was no difference in fungal species richness $\left(\mathrm{F}_{1,22}=0.60, \mathrm{P}=0.45\right)$ as affected by plot history. The mean fungal species richness as determined by spore morphology in plots with a $B t$ vs P history was 3.52 and 3.67 , respectively.

Effect of Bt maize on AMF colonization

There was no difference in colonization by AMF hyphae $\left(\mathrm{F}_{1,6}=0.08, \mathrm{P}=0.78\right)$, arbuscules $\left(\mathrm{F}_{1,6}=0.02, \mathrm{P}=0.90\right)$, vesicles $\left(\mathrm{F}_{1,6}=0.21, \mathrm{P}=0.66\right)$, or total percentage AMF colonization $\left(\mathrm{F}_{1,6}=0.06, \mathrm{P}=0.81\right)$ between $B t$ and non- $B t$ maize at the time of harvest (Fig. 13). Mean AMF colonization levels in split plots 60 days after sowing were $72.68 \%$ in $B t$ maize and $72.16 \%$ in non- $B t$ maize.

Effect of AMF colonization and cultivar on maize growth

AMF colonization was negatively correlated with root biomass $\left(\mathrm{F}_{1,273}=6.15, \mathrm{P}=\right.$ $0.01)$ and leaf chlorophyll content $\left(\mathrm{F}_{1,273}=4.46, \mathrm{P}=0.035\right)$, but there was no effect of AMF on shoot biomass $\left(\mathrm{F}_{1,273}=1.47, \mathrm{P}=0.23\right)$. Initial size was positively correlated with root biomass $\left(\mathrm{F}_{1,273}=109.95, \mathrm{P}<0.0001\right)$, shoot biomass $\left(\mathrm{F}_{1,273}=787.68, \mathrm{P}<0.0001\right)$, and leaf chlorophyll content $\left(\mathrm{F}_{1,273}=5.19, \mathrm{P}<0.02\right)$. Chlorophyll content in leaves was positively correlated with root biomass $\left(\mathrm{F}_{1,273}=108.71, \mathrm{P}<0.0001\right)$ and shoot biomass 
$\left(\mathrm{F}_{1,273}=120.14, \mathrm{P}<0.0001\right)$.

There was no difference in root biomass $\left(\mathrm{F}_{1,6}=3.48, \mathrm{P}=0.11\right)$, shoot biomass $\left(\mathrm{F}_{1,6}=\right.$ $1.52, \mathrm{P}=0.26)$, or chlorophyll content $\left(\mathrm{F}_{1,6}=0.38, \mathrm{P}=0.56\right)$ between the $B t$ and non- $B t$ maize cultivars at the time of harvest; mean root biomass was $3.06 \mathrm{~g}$ in $\mathrm{Bt}$ maize and 2.65 $\mathrm{g}$ in non- $B t$ maize; mean shoot biomass was $29.01 \mathrm{~g}$ in $B t$ maize and $28.17 \mathrm{~g}$ in non- $B t$ maize; and mean 60 day leaf chlorophyll content was 42.71 in $B t$ maize and 42.50 in nonBt maize.

Effect of plot history on AMF colonization and plant growth

$B t$ plants grown in $B t$ plots had higher leaf chlorophyll content at the time of harvest than $B t$ plants grown in $\mathrm{P}$ plots, and vice versa $\left(\mathrm{Bt} *\right.$ history $\mathrm{F}_{1,38}=4.44, \mathrm{P}=0.04$; Fig. 14). However, there was no effect of plot history $(B t$ or $\mathrm{P})$ on AMF colonization $\left(\mathrm{F}_{1,38}=0.33\right.$, $\mathrm{P}=0.57)$, initial size $\left(\mathrm{F}_{1,38}=1.25, \mathrm{P}=0.07\right)$, initial chlorophyll content $\left(\mathrm{F}_{1,38}=1.09, \mathrm{P}=\right.$ $0.30)$, root biomass $\left.\mathrm{F}_{1,38}=3.46, \mathrm{P}=0.07\right)$, or shoot biomass $\left(\mathrm{F}_{1,38}=1.59, \mathrm{P}=0.21\right)$.

\section{Discussion}

This study presents the first evidence of an effect of $B t$ maize cultivation on the soil ecosystem, but also provides further evidence that this effect is not necessarily large or easily detectable within the range of normal environmental variation. The strength of our approach is that we cultivated seven different $B t$ maize genotypes and five corresponding parental $(\mathrm{P})$ isolines over two growing seasons, making this the most comprehensive 
study to date examining potential nontarget effects of $B t$ maize in the field. We found that plots with a non- $B t$ maize cultivation history had higher numbers of total spores at the beginning of the field season compared to plots with a $B t$ maize history, indicating a potential negative effect of $B t$ maize cultivation on AMF propagules in the soil over time. We also detected a positive feedback fitness effect whereby Bt plants grown in Bt plots had higher leaf chlorophyll content at the time of harvest than Bt plants grown in P plots, suggesting that plot history may have an impact on nutrient status of subsequently planted crops. However, we found no differences in AMF colonization, root, or shoot biomass between plant type ( $B t$ or non- $B t$ maize) or as affected by cultivation history. Because we used the same maize genotypes as in previous greenhouse (Cheeke et al. 2011, Cheeke et al. 2012) and field experiments (Cheeke et al. 2013), we can now make predictions on how $B t$ maize cultivation may affect AMF under different environmental conditions over time.

Our greenhouse experiments demonstrated a reduced level of AMF colonization in Bt maize and revealed that these differences in colonization were greatest when spore density was high and fertilizer applications were absent or limited (Cheeke et al. 2011, Cheeke et al. 2012). This is important because these are the environmental conditions where AMF would be of most benefit to plant health and fitness. In the present study, we detected no differences in AMF colonization between $B t$ and non-Bt maize, even though field plots with a non-Bt cultivation history had higher spore numbers at the beginning of 
the season. Assessing sporulation at the beginning, as well as at the end of the growing season could be a strong measure of fungal fitness than colonization (Bever 2002), although both are important. While these results contradict greenhouse studies (Castaldini et al. 2005, Cheeke et al. 2011, Cheeke et al. 2012), they support those of our 2009 field study where we also found no differences in AMF colonization between $B t$ and non- $B t$ maize (Cheeke et al. 2013). Potential reasons for this include differences in soil type, mycorrhizal communities, and the heterogeneous soil conditions in the field that make differences in AMF colonization between $B t$ and $\mathrm{P}$ maize difficult to detect. Soil nutrient analysis revealed that our field site contained moderate levels of nitrogen and high levels of available phosphorous, which were higher than those in our greenhouse studies. Taken together, these results suggest that differences in AMF colonization between $B t$ and non$B t$ maize may be more apparent under field conditions where soil nutrients are limited.

We detected a positive feedback effect whereby $B t$ plants grown in $B t$ plots had higher leaf chlorophyll content than Bt plants grown in P plots; similarly, non-Bt parental plants had higher leaf chlorophyll content when grown in plots previously cultivated with self. Because both $B t$ and non- $B t$ maize genotypes were grown together in the same splitplot, it is unlikely that differences in soil chemistry could account for differences in leaf chlorophyll content, as the plants shared the same nutrient microhabitat. This positive feedback effect may be driven by differences in microbial communities in each plot; AMF are known to confer different benefits to plants depending on their taxonomic 
identity (van der Heijden et al. 1998, Lendenmann et al. 2011) and plants have also been shown to favor AMF that provide higher benefits to the plant (Bever et al. 2009, Kiers et al. 2011). Thus, it is possible that the specific AMF and/or microbial community in each plot could be interacting with $B t$ and non- $B t$ maize plants in different ways, conferring unique nutrient benefits to their specific plant host. However, AMF community composition did not differ between four different $B t$ and non-Bt maize cultivars in a greenhouse study (Verbruggen et al. 2012). Because we included 14 different $B t$ and nonBt genotypes in our study, it is possible that there may be some specific plant genotype $\mathrm{x}$ fungal interactions influencing the positive feedback effect we observed, however this remains to be tested.

Although AMF colonization was not correspondingly lower in plots with a $B t$ history, our study indicates that fields with long-term $B t$ maize cultivation may lead to a lower number of AMF spores in the soil over time. Reduced numbers of AMF propagules in the soil could potentially have an effect on soil ecosystem services including carbon sequestration (Six et al. 2006), nutrient cycling (Whiteside et al. 2009, Veresoglou et al. 2012), drought tolerance (Auge 2001, Barzana et al. 2012), soil aggregation (Rillig 2004), and plant resistance to pathogens (Wehner et al. 2011, Jung et al. 2012), however this remains to be tested on a longer timescale. Lower AMF spore numbers in commercial maize fields are not likely to affect crop performance or yield (most fields are fertilized and irrigated), but may be of importance in low-input systems (Hooker and 
Black 1995, Harrier and Watson 2003, Jeffries et al. 2003), crop rotation regimes (Johnson et al. 1991, Gavito and Miller 1998), and grassland restorations (McCain et al. 2011, Middleton and Bever 2012).

In future studies, characterization of AMF communities in roots and soil would help to elucidate the mechanism for higher leaf chlorophyll content in $B t$ and non-Bt maize plants grown in plots previously cultivated with self. This positive feedback fitness effect is particularly interesting as there was no difference in AMF colonization of roots between $B t$ or non- $B t$ maize and no difference in colonization as affected by plot history. Determining AMF identity may be important as different taxa have been shown to confer different benefits to plants depending on their taxonomic or functional identity (Jakobsen et al. 1992, Munkvold et al. 2004, Jansa et al. 2005, Lendenmann et al. 2011, Thonar et al. 2011). Plots with a non-Bt parental cultivation history had higher spore numbers compared to plots with a $B t$ cultivation history at the beginning of the field season, so spore number, as well as fungal identity, may be important as plants establish symbioses with AMF early in the field season. Additional field studies should be conducted to see what effects, if any, the cultivation of $B t$ maize might have on symbiotic arbuscular mycorrhizal fungi in the soil ecosystem over a longer timescale. 


\section{Chapter 6. Exploring potential mechanisms for lower AMF colonization in Bt maize}

Authors: Tanya E. Cheeke, Corey R. Guidry, Luke Reyes, Erik Hasenkopf, Ann Rasmussen, Mitchell B. Cruzan, and Todd N. Rosenstiel.

\section{Introduction}

Genetically modified (GM) maize has been grown commercially since 1996 and now constitutes $88 \%$ of all maize grown in the United States (USDA 2012). One of the most widely planted GM crops, Bacillus thuringiensis (Bt) maize releases an insecticidal toxin that binds to soil particles and remains biologically active in the environment for at least several months (Tapp and Stotzky 1995b, a, Palm et al. 1996, Tapp and Stotzky 1997, 1998, Zwahlen et al. 2003). Previous studies revealed an altered mycorrhizal relationship in different lines of $B t$ maize (Turrini et al. 2004, Castaldini et al. 2005, Cheeke et al. 2011, Cheeke et al. 2012), however these studies did not identify a proximal mechanism. One potential mechanism for lower AMF colonization in $B t$ maize may be alterations in root invertase activity at the plant-fungal interface. Invertase is a key enzyme for AMF establishment in plant roots; it cleaves sucrose from the photosynthate into glucose and fructose hexoses at the apoplastic interface to support the fungal mutualism (Schaarschmidt et al. 2006, Garcia-Rodriguez et al. 2007). Arbuscular mycorrhizal fungi appear to lack invertase enzymes as intraradical AMF structures have been shown to only take up hexoses (primarily glucose) in studies using isotopic labeling with NMR spectrometry in colonized roots (Shacharhill et al. 1995, Pfeffer et al. 1999) and in 
experiments using radiorespirometry measurements on isolated intraradical hyphae (Solaiman and Saito 1997, reviewed by Ferrol et al. 2002). Thus, examining differential invertase activity in the roots of $B t$ and non- $B t$ maize may provide insights to the underlying mechanism driving the reduced AMF colonization in Bt plants. Given that pleiotropic effects (change in one gene that influences other phenotypic traits) have been observed in multiple $B t$ crop plants (Saxena and Stotzky 2001, Flores et al. 2005), it is possible that enzymatic activity could be altered in the $B t$ maize as well.

Other potential mechanisms for lower AMF colonization in $B t$ maize include pleiotropic effects that may lead to reduced root permeability driven by increased levels of suberin or lignin content. Arbuscular mycorrhizal fungi colonize plants by entering primarily through the fine roots. Upon contact, AMF hyphae enter through passage cells in the hypodermis of dimorphic roots (Sharda and Koide 2008, Smith and Read 2008). These passage cells (also called 'short cells') lack suberin lamellae (a barrier forming lipid that limits water and ion transfer; and are more abundant in younger or secondary roots (Zadworny and Eissenstat 2011). As the root develops, the passage cells become suberized and do not allow AMF penetration. Thus, reduced root permeability in $B t$ maize (due to increased suberin content) could represent a potential mechanism for the lower AMF colonization observed in greenhouse studies. Multiple $B t$ crops have also been shown to have higher lignin content in stem and leaf tissue (Saxena and Stotzky 2001, Flores et al. 2005), but it is not known whether $B t$ plants also exhibit higher lignin 
in roots. If $B t$ maize roots have a higher lignin content compared to their non- $B t$ parental isoline, this may represent a mechanical barrier to AMF establishment by limiting root permeability. Higher lignin content may contribute to lower decomposition rates in the field over time (Flores et al. 2005), potentially affecting nutrient cycling and impacting soil microbial communities.

Pre-symbiotic barriers may contribute to the reduced AMF colonization observed in $B t$ maize. Pre-symbiosis includes spore germination, recognition of host, and appressoria development (Smith and Read 2008). Once a spore germinates, hormones in root exudates (i.e. strigolactones) stimulate AMF hyphae to branch and grow towards plant roots (Akiyama et al. 2005, Akiyama and Hayashi 2008). Experiments using semipermeable membranes with spores physically separated from roots of a mycorrhizal host plant, a non-host, and dead roots, demonstrated that AMF did not grow towards non-host plants or dead roots, but did branch and grow toward host plants when exposed only to root exudates (Sbrana and Giovannetti 2005). Using a similar semi-permeable membrane technique, Turrini et al. found that root exudates of $B t$ maize ( $B t 176)$ reduced presymbiotic hyphal growth, thus negatively affecting normal AMF development in the $B t$ maize (Turrini et al. 2004). As Bt proteins do not have a direct effect on AMF (Ferreira et al. 2003), this suggests a pleiotropic effect whereby root exudates were altered in a $B t$ genotype compared to its non- $B t$ parental cultivar as a result of a genetic insertion. After spore germination and chemotaxic growth of hyphae towards root exudates, 
appressoria (club-shaped early AMF infection structures) form on the epidermis of plant roots. After appressoria are formed at the plant-fungal interface, infection pegs begin to develop, and if successful, the symbiosis is established and arbuscules form within plant cells. It has been demonstrated that appressoria were reduced in at least one line of $B t$ maize (Bt 176) whereby $36 \%$ of appressoria failed to develop viable infection pegs (Turrini et al. 2004). However, there was no effect of Bt 11 maize on appressorium development or the viability of infection pegs (Turrini et al. 2004). It has also been demonstrated that fertilizer additions (especially P) can limit AMF pre-symbiotic development and reduce hyphal branching (Nagahashi et al. 1996), so it is possible that alterations to fertilizer level may affect appressoria formation or the viability of infection pegs in some $B t$ maize lines.

In a set of three exploratory laboratory experiments, we aimed to develop an understanding of the mechanisms that both enable and limit AMF colonization in $B t$ maize, with the overall goal of determining whether $B t$ crop cultivation has an inhibitory effect on AMF in the soil. The three potential mechanisms we investigated include differences in: 1) invertase activity, 2) root permeability (suberin and lignin content), or 3) pre-symbiotic barriers including the number of aborted infection pegs, appressoria, and passage cells in $B t 11$ versus non- $B t$ maize roots. In the first experiment, we asked the question: Is there is difference in acid invertase activity in the roots of Bt and non-Bt maize? If so, do plants with lower invertase activity have correspondingly lower AMF 
colonization in roots? We hypothesized that Bt 11 maize would have lower invertase activity and that this would correspond with lower AMF colonization levels. In the second experiment we aimed to determine whether there are any physical barriers to mycorrhizal penetration in Bt maize roots. Specifically, we asked: Do Bt maize roots have higher suberin or lignin content than non-Bt maize roots? We hypothesized that Bt maize would have greater suberin lamellae deposition and higher lignin content in the cortical cells of roots compared with a non-Bt parental isoline. In the third experiment, we investigated potential pre-symbiotic barriers to colonization of $B t 11$ maize. Here, we asked: Do Bt maize plants have lower numbers of appressoria, higher numbers of aborted infection pegs, or fewer passage cells in roots than their corresponding non-Bt parental isoline? If so, how is this affected by fertilizer addition? We hypothesized that $B t$ maize would have lower numbers of appressoria, fewer passage cells, and more aborted infection pegs than the parental cultivar and that plants grown in the absence of chemical fertilizer would have more appressoria and passage cells than plants grown with low amounts of fertilizer.

\section{Materials and Methods}

\section{Plant material}

Experiments were performed using Zea mays (triple sweet hybrid sweet maize, $B t$ 11; Attribute) transformed to express the CrylAb protein of Bacillus thuringiensis and its non-transgenic parental isoline (Providence). Seeds were obtained from Syngenta Seeds 
Inc. (Boise, ID). Bt 11 was shown to have lower levels of AMF colonization in a previous greenhouse experiment (Cheeke et al. 2011) and thus was established to be a good candidate for preliminary explorations into mechanisms that may be limiting AMF colonization in $B t$ maize.

\section{Root invertase activity}

Sucrose cleavage in $B t$ and non-Bt maize plants was evaluated by an in vivo measurement of acid invertase activity in intact fine root pieces of each plant type. $B t$ and non- $B t$ maize plants were grown for 30 days in potting soil (without the addition of fertilizer or mycorrhizal spores) in the greenhouse. The root system of each living whole plant was soaked in a weak buffer solution (3 parts deionized water: 1 part MES 2-(Nmorpholino) ethanesulfonic acid]) for 24 hours to wash endogenous sugars from the roots. After soaking, $50 \mathrm{mg}$ root samples were chopped into approximately $1 \mathrm{~mm}$ segments and placed in $1.5 \mathrm{ml}$ Eppendorf screw cap tubes, suspended in $1 \mathrm{ml}$ buffer $(0.2$ M MES titrated with $\mathrm{NaOH}$ to $\mathrm{pH} 4.8$ ) and $0.25 \mathrm{ml}$ of $0.1 \mathrm{M}$ sucrose solution. Samples were placed in a heat block $\left(45^{\circ} \mathrm{C}\right)$ for 15 and 30 minute spans. After incubation, the root segments were removed and the remaining solution was boiled for 15 minutes to halt enzymatic activity. The DNS method (Deng and Tabatabai 1994) was used to determine reducing sugar concentration in the solution. $0.5 \mathrm{ml}$ of DNS reagent was combined with $0.5 \mathrm{ml}$ of the boiled root solution, vortexed, and boiled for 15 minutes to develop the characteristic red-brown color. Sample absorbance was determined at OD $540 \mathrm{~nm}$ 
(Shimadzu 1201) and reducing sugar concentration compared to an authentic standard curve (glucose). Samples containing cut roots and MES buffer were allowed to incubate without addition of the sucrose substrate for 30 minutes to use as a control to measure background reducing sugar content. Experiments were performed at different $\mathrm{pH}$ levels (pH 5.5 and $\mathrm{pH} 8.0)$ to analyze acid invertase versus alkaline invertase and sucrose synthase activity. Differences in invertase activity at the 15 and 30 minute incubation times for $B t$ and non-Bt maize roots were analyzed in two separate one-way ANOVA models (SAS, version 9.1).

\section{Root permeability}

$B t$ and non- $B t$ maize plants were grown in a greenhouse for approximately three weeks in $10 \%$ whole soil (from pot cultures developed from field soil, Corvallis, OR) and 1:1 mixture of sterile sand and potting mix. Plants were harvested when AMF colonization first became measureable (when plants were about $12 \mathrm{~cm}$ tall). Soil was rinsed from roots and fresh cross sections were taken from adventitious roots, stained for approximately four minutes with one of a variety of tissue-specific stains (Table 18), mounted on microscope slides, and viewed using a stereomicroscope (Leica MZ16) using both white light and epifluorescent light. Observable differences in root architecture, lignin, or suberin between $B t$ and non- $B t$ cross-sectioned roots were recorded for each sample. Five to ten cross-sections from varying distances from the root tip were observed for each individual plant. Differences in lignin and suberin between $B t$ and non-Bt maize 
were assessed visually by viewing stained specimens under the microscope using the same light intensity and magnification among samples.

Aborted infection pegs, appressoria, and passage cells

Assessment of appressoria, infection pegs, and passage cells was conducted using slides from a previous greenhouse study where $B t$ maize was shown to have lower levels of AMF colonization (Cheeke et al 2011). Differences in aborted infection pegs, appressoria, and passage cells were assessed between $B t$ and non-Bt maize inoculated with 80 spores of Glomus mosseae and fertilized weekly with 'No' or 'Low' fertilizer levels. Using the slide-intersect method, the presence/absence of aborted infection pegs, appressoria, and passage cells were recorded per 100 intersects (McGonigle et al. 1990). Plants in the 0 and 40 spore treatments, as well as those in the high fertilizer treatment, were eliminated from this analysis because they had very little AMF colonization and there was no difference in colonization between $B t$ and non- $B t$ maize plants (Cheeke et al. 2011). Differences in aborted infection pegs, appressoria, and passage cells between $B t$ and $\mathrm{P}$ plants in the No and Low fertilizer treatments were evaluated using t-tests (Excel, Windows 2010); $\mathrm{n}=5 \mathrm{Bt}$ plants and $5 \mathrm{P}$ plants per treatment.

\section{Results}

Root invertase activity

Cell wall acid invertase activity was assayed in uncolonized $B t$ and non-Bt maize 
roots. An in vivo assay for acid invertase activity was chosen as it reflects the root condition present during AMF colonization. We found that intact, uncolonized non- $B t$ maize roots displayed twice the invertase activity of $B t$ maize roots at both incubation times (Fig. 15; 15 min: $F=27.19$, df $=1 ; 30 \mathrm{~min}: \mathrm{F}=22.39$, $\mathrm{df}=1$ ). No measurable level of background reducing sugar was detected in control samples containing only root segments and MES buffer, nor was any activity observed at higher assay $\mathrm{pH}$ conditions $(\mathrm{pH} 8.0)$.

Root permeability: Lignin and suberin content in roots

We observed no differences in lignin content in root sections of $B t$ vs non- $B t$ maize stained with Toluidine Blue. The xylem of both plant types stained dark blue using this stain (Fig. 16). Fresh cross sections from roots stained with Safranin O showed minimal differences in cortical lignin content between $B t$ and non- $B t$ maize, and the exodermis and root segments stained similarly in both the Bt and P line (Fig. 16). When stained with Safranin O, the $B t$ maize roots appeared to stain more brightly, but this wasn't always consistent across samples. We detected no differences in suberin or lignin in $B t$ and non-Bt maize roots stained with Berberine Hemi-Sulfate and Analine Blue or between $B t$ and non-Bt maize stained with Sudan III to detect suberin (Fig. 16). Pre-symbiotic barriers: Aborted infection pegs, appressoria, and passage cells

Despite lower AMF colonization in the Bt 11 maize plants in the 'No' and 'Low' fertilizer treatments in an earlier study (Cheeke et al. 2011) (Fig. 1), we detected no 
significant differences in aborted infection pegs between $B t$ and non- $B t$ maize roots $(\mathrm{p}=$ 0.30). The mean number of aborted infection pegs in the 'No fertilizer treatment was 3.0 in the $B t$ plants and 10.4 in the P plants $(\mathrm{p}=0.20$; Fig. 17); the mean number of abortive infection pegs in the 'Low' fertilizer treatment was 0.8 in the $B t$ plants and 4.2 in the $\mathrm{P}$ plants (Fig. 17). There were no also differences in the number of appressoria between $B t$ and non- $B t$ maize $(\mathrm{p}=0.73)$. The mean number of appressoria in the 'No fertilizer treatment was 2.6 in the $B t$ plants and 1.8 in the P plants (Fig. 17). The mean number of appressoria were the same between the $B t$ and P plants in the 'Low' fertilizer treatment $($ mean $=0.2$ appressoria per 100 intersects; Fig. 17).

Interestingly, Bt plants in the No fertilizer treatment had more passage cells than $\mathrm{P}$ plants ( $p=0.03$; Fig. 17), even though they had lower levels of AMF colonization (Fig. 1). The mean number of passage cells in the 'No' fertilizer treatment was 1.4 in $B t$ plants and 0.2 in the non-Bt parental plants. There was no difference in mean passage cell number between $B t$ and $\mathrm{P}$ in the Low fertilizer treatment $(\mathrm{p}=0.37)$. The mean number of passage cells in the 'Low' fertilizer treatment was 0.2 in the $B t$ plants and 0 in the non- $B t$ parental plants (Fig. 17). Overall, the number of aborted infection pegs, appressoria, and passage cells were higher in the 'No' fertilizer treatments than in the 'Low' fertilizer treatment in both $B t$ and non- $B t$ maize plants (Fig. 17). 


\section{Discussion}

In an effort to identify potential mechanisms for the reduction in AMF colonization observed in Bt maize (Cheeke et al. 2011, Cheeke et al. 2012), we investigated differences in root enzyme activity, root permeability, and pre-symbiotic barriers in $B t 11$ maize and its non-Bt parental isoline. Acid invertase was chosen as an important enzyme to investigate because it plays a key role in the establishment and regulation of the AMF mutualism at the plant-fungal interface (Schaarschmidt et al. 2007b). Lignin and suberin were selected because high levels of either compound may inhibit AMF colonization by reducing root permeability at the plant-fungal interface (Smith and Read 2008). The number of aborted infection pegs, appressoria, and passage cells were evaluated in $B t$ and non- $B t$ roots to examine potential pre-symbiotic barriers that may limit AMF colonization in $B t 11$ maize. In this study, we found that Bt 11 maize had lower levels of invertase activity and more passage cells in roots than their non-Bt parental isoline. We also found that although there weren't many observable differences between suberin and lignin content in $B t$ versus non- $B t$ maize roots, $B t$ roots stained with Safranin 0 appeared to have higher lignin content than the $\mathrm{P}$ isoline, but further tests are required to confirm this. There were no differences in appressoria or aborted infection pegs detected between the $B t$ and non- $B t$ maize roots, however both structures occurred in higher numbers in the 'No' fertilizer treatment than in the 'Low' fertilizer treatment.

In our first experiment, we analyzed basal acid invertase activity in roots of 
uninoculated $B t$ and non- $B t$ maize. Although upregulation of a cell wall invertase gene in the plant host is required for carbon transfer across the apoplastic interface to support the fungal symbiont (Schaarschmidt et al. 2006, Garcia-Rodriguez et al. 2007, Schaarschmidt et al. 2007a, Schaarschmidt et al. 2007b), basal invertase activity should provide an indication of the potential of roots to attract and support AMF colonization. Using an in vivo assay, we found that uncolonized non- $B t$ plants exhibited twice as much invertase activity as the $B t 11$ maize plants. This is significant because reduced invertase activity in $B t$ plant roots may be a principal mechanism for the lower AMF colonization levels. It is still not clear, however, whether this reduction in basal invertase activity is a result of reduced invertase expression or possible inhibition of enzyme activity by pleiotropic impacts on root metabolism or the soil environment. Regardless of the mechanism of reduced invertase activity in the $B t$ isoline, these results are consistent with the emerging view that acid invertase may be a key to understanding AMF colonization in many plant systems. This finding also corresponds with lower AMF colonization detected in the $B t$ 11 maize line in a previous study (Cheeke et al. 2011).

Lignin and suberin content were evaluated in the roots of $B t$ and non-Bt maize inoculated with AMF from field soil. Plants with thicker, less permeable roots may lead to reduced AMF colonization by preventing or inhibiting early AMF infection structures. Although we noticed that the $B t$ roots were physically more difficult to cut when making cross-sections compared to the non- $B t$ parental roots, we were not able to detect 
consistent differences in suberin or lignin content in roots with the stains used in this study. However, $B t$ roots stained with Safranin O appeared to stain much brighter than the $\mathrm{P}$ roots, potentially indicating a higher lignin content in cortical cells in Bt 11 maize roots. This supports other research showing higher lignin content in the shoots of $B t$ maize (Saxena and Stotzky 2001). We specifically examined differences in lignin and/or suberin content in cortical cells or on the epidermis of roots to examine potential differences in root permeability at the plant-fungal interface. Previous studies quantified lignin after depolymerization (BF3/methanol-transesterification, thioacidolysis) using gas chromatography/mass spectrometry (Zeier and Schreiber 1997, 1998), however, this only provides total lignin content. As most of the lignin in plant roots is in the xylem where AMF do not colonize, we chose to use tissue-specific stains instead to visualize potential differences on the epidermal layer of roots.

To examine potential pre-symbiotic barriers to AMF colonization, we used slides from a previous study in which Bt 11 maize had lower levels of AMF colonization compared to the non-Bt maize isoline (Cheeke et al. 2011) and quantified the number of aborted infection pegs, appressoria, and passage cells in each sample. High numbers of aborted infection pegs (devoid of protoplasm) can indicate failed infection attempts (Turrini et al. 2004, Smith and Read 2008); overproduction of appressoria may be a fungal response to failure of tissue colonization indicating ongoing attempts to breach defenses (Smith and Read 2008); and the number of passage cells may influence the 
ability of AMF to colonize roots. Although we detected no difference in aborted infection pegs or appressoria between $B t$ and P maize roots, we found (somewhat paradoxically) that $B t$ plants had more passage cells than P plants, but lower AMF colonization in the 'No' fertilizer treatment. It is interesting that this pattern disappears once small amounts of fertilizer were added in the 'Low' fertilizer treatment.

Future studies would benefit from including more genotypes of $B t$ and non- $B t$ maize, as well as plants (and roots) of varying ages. Lignin content in stems and leaves, for example, is known to vary among cultivars, and can also differ by plant age (Icoz and Stotzky 2008). It is likely that invertase activity and pre-symbiotic barriers could vary with genotype and plant age as well. Quantifying lignin and suberin content in cortical and epidermal cells proved to be difficult with our staining techniques, so developing a refined method for this would be ideal. Investigating potential differences in root exudate profiles, volatile organic compounds in roots, and $B t$ protein concentrations in the rhizosphere of different $B t$ maize genotypes would also help to elucidate possible mechanisms for the reduced AMF colonization observed in $B t$ maize. 


\section{Chapter 7. Conclusions, future directions, and broader impacts}

Prior to this PhD work, very few studies had investigated the effects of $B t$ crop cultivation on symbiotic arbuscular mycorrhizal fungi (AMF). This is surprising because AMF appear to be an ideal system to investigate nontarget effects of transgenic crops. AMF are obligate symbionts that require a plant host for nutrition and reproduction, and live within the cortical tissue of roots. Thus, they may be more sensitive to genetic changes within a plant or to changes in the rhizosphere than other free-living organisms in the soil. Indeed, previous studies demonstrated an altered relationship between $\mathrm{Bt}$ maize and AMF in greenhouse experiments (Turrini et al. 2004, Castaldini et al. 2005), but until this dissertation project, no field studies had been conducted to evaluate the effects of $B t$ maize on AMF, and potential mechanisms contributing to lower AMF colonization in $B t$ maize remained largely unexplored.

Because of the dearth of information related to nontarget effects of $B t$ crop cultivation on symbiotic soil fungi, and the importance of AMF to soil, plant, and ecosystem health, I designed a comprehensive set of greenhouse and field experiments to address the following integrated questions:

1) What are the experimental and environmental factors contributing to differences in AMF colonization in $B t$ and non-Bt plants reported in the literature? 
2) Will AMF colonization be reduced in multiple $B t$ maize lines when grown under the same experimental conditions in a greenhouse, and if so, are these differences are related to the expression of a particular $B t$ protein?

3) Does $B t$ maize cultivation have a negative effect on AMF colonization of a subsequently planted crop species?

4) Is AMF colonization reduced in $B t$ maize under field conditions?

5) Will the cultivation of $B t$ maize have a negative effect on the abundance and diversity of AMF in the roots and rhizosphere over time?

6) Are there fitness-related feedback effects due to $B t$ or non- $B t$ maize cultivation history?

7) What are some potential mechanisms that may lead to reduced AMF colonization in $B t$ maize?

To address these questions, I used seven different genotypes of $B t$ maize and five corresponding non- $B t$ parental isolines obtained from three different seed companies (Monsanto Co., Syngenta, and a company that prefers to remain anonymous) in a series of greenhouse and field experiments. The maize genotypes in my collection represented a cross-section of the $B t$ maize cultivars commercially available and varied in the number and type of engineered trait, as well as background genetics.

\section{Conclusions and future directions}

My dissertation research has shown that AMF colonization is reduced in multiple 
genotypes of Bt maize (Cheeke et al. 2012), but only under certain ecological conditions. My greenhouse studies demonstrated that the greatest difference in AMF colonization between $B t$ and P maize was observed when spore density was high and fertilizer additions were low or absent (Cheeke et al. 2011). This is significant because these are the ecological conditions where AMF can be of most benefit to the plant; AMF increase the surface area of roots and help the plant to obtain essential nutrients such as $\mathrm{N}$ and $\mathrm{P}$, as well as improve water uptake during times of drought stress (Smith and Read 2008). While we did not detect any difference in AMF colonization between $B t$ and $\mathrm{P}$ maize in the field (Cheeke et al. 2013; Ch. 5), it does not mean the effects were not there. Spatial heterogeneity of AMF propagules and differences in nutrient availability in the field make significant effects difficult to detect, especially when plants are grown in highly fertile soils. Even though I did not fertilize my field plots (to mimic the nutrient stress that exacerbated differences in AMF colonization between $B t$ and $\mathrm{P}$ maize in greenhouse experiments), the field site in the Willamette Valley of Western Oregon was naturally nutrient-rich and plants grew with vigor. Soil nutrient analysis also confirmed that the field site had higher levels of $\mathrm{N}$ and $\mathrm{P}$ than soil used in my greenhouse experiments. The field site almost certainly had a different AMF community than that of the Vancouver, WA soil that was used as inocula in my greenhouse study. Future studies would benefit from examining AMF colonization in a variety of $B t$ and non- $B t$ maize genotypes grown in a range of soil conditions in the field. Effects would likely be most pronounced in low- 
input agricultural systems, areas where nutrients have been depleted, or during conditions of drought stress.

It is curious why $B t$ maize would have lower AMF colonization compared to their non-Bt parental isolines under low nutrient conditions. Because $B t$ maize constitutively expresses one or more $B t$ insecticidal proteins, one would think that the nutrient requirements (especially $\mathrm{N}$ ) would be greater in the $B t$ cultivars. Correspondingly, it would make sense that $B t$ plants would have an increased reliance on AMF to meet their nitrogen needs. However, multiple greenhouse studies from independent labs have demonstrated an altered relationship between Bt maize and AMF (Turrini et al. 2004, Castaldini et al. 2005, Cheeke et al. 2011, Cheeke et al. 2012) under a range of experimental conditions. The potential mechanisms contributing this altered relationship are still unresolved. Although I examined a variety of possible mechanisms contributing to lower AMF colonization in $B t$ maize, none of my experiments were unequivocally conclusive. Future experiments should continue to investigate potential differences in apoplastic invertase activity in roots, root physiology (i.e. lignin or suberin content), and pre-symbiotic barriers in a variety of $B t$ and non- $B t$ plant species, and would also benefit from examining possible differences in root exudate profiles and volatile organic compounds in roots that may be important for plant/AMF signaling. Investigating potential correlations between the amount of $B t$ protein expressed in plant roots and AMF colonization would also be beneficial - it is possible that effects on AMF vary by $B t$ 
protein expression levels in the rhizosphere, as was demonstrated by Turrini et al. (2004) whereby $B t 176$ had a greater negative effect on AMF than $B t 11$, and also had higher $B t$ protein expression. This remains to be tested across a broader range of $B t$ genotypes.

\section{Significance/Broader Impacts}

Previous studies on the environmental impacts of genetically engineered crops in soil have focused primarily on the soil microbial community; however alterations in bacterial community structure are difficult to link to ecological significance and are often difficult to distinguish from natural fluctuations in the soil environment. By focusing on AMF, I was able to evaluate the impact of $B t$ plants on a nontarget fungal symbiont that often confers nutrient and water benefits to plants (Smith and Read 2008) and investigate whether the reduction in AMF observed in Bt maize may be due to intrinsic factors (i.e. alterations in root enzymes, changes in root permeability) in $B t$ maize or due to environmental variability in soil or growing conditions. Though it would have been ideal to include transgenic maize varieties that express only herbicide tolerance or some other non- $B t$ trait to examine the effects of a non- $B t$ genetic insertion on AMF, these seeds were not provided by the seed companies as non- $B t$ transgenic maize varieties are rarely grown in the commercial marketplace (Syngenta, Monsanto, personal communication). Field trials allowed me to develop an understanding of the nontarget effects of transgenic $B t$ crop cultivation on symbiotic mycorrhizal fungi in the soil ecosystem and to determine whether $B t$ crop cultivation has an inhibitory effect on AMF abundance and diversity in 
the soil over multiple growing seasons. A general reduction in the density or diversity of AMF propagules in the soil may impact soil structure and function over time. This would not only impact current and future crop plantings, but may also hinder the ability of grassland plants to re-colonize former agricultural fields and represents a potentially significant unintended consequence of $B t$ crop cultivation on soil ecosystems. There is also the potential for the effects of $B t$ transgenes to extend beyond agricultural systems as introgression of $B t$ transgenes into traditional landraces in Mexico has recently been reported (Pineyro-Nelson et al. 2009). It is not clear how Bt genes will affect AMF colonization in these plants but as more and different types of $B t$ crops are developed, it is important to determine the effects of the $B t$ transformation on the physiology of the plant and its ability to interact with symbiotic organisms in the rhizosphere.

Results from my combined experiments provide a comprehensive assessment of the impact of $B t$ plants on symbiotic soil organisms across a broad range of environmental and ecological conditions and has helped to elucidate possible mechanisms that influence AMF colonization in $B t$ maize. My hope is that this research will contribute to the development of future biotech risk assessment protocols to minimize nontarget effects of $B t$ crops on symbiotic fungi, and will help to expand the breadth of knowledge surrounding these crops. Findings from this study have been published in the scientific literature and presented annually at scientific meetings. This project also provided interdisciplinary research training for at least 20 student researchers including four high 
school students, two honors thesis students, and one McNair Scholar. I intend to continue this tradition of mentoring and incorporating students into my research as I continue through my academic career. 


\section{Tables and Figures}

Table 1. Global area of genetically modified crops in 2010: By country (million ha)

\begin{tabular}{|c|c|c|c|}
\hline Rank & Country & $\begin{array}{l}\text { Area (million } \\
\text { hectares) }\end{array}$ & Biotech crops \\
\hline 1 & USA & 66.8 & $\begin{array}{l}\text { Maize, soybean, cotton, canola, sugarbeet, alfalfa, } \\
\text { papaya, squash }\end{array}$ \\
\hline 2 & Brazil & 25.4 & Soybean, maize, cotton \\
\hline 3 & Argentina & 22.9 & Soybean, maize, cotton \\
\hline 4 & India & 9.4 & Cotton \\
\hline 5 & Canada & 8.8 & Canola, maize, soybean, sugarbeet \\
\hline 6 & China & 3.5 & Cotton, tomato, poplar, papaya, sweet pepper \\
\hline 7 & Paraguay & 2.6 & Soybean \\
\hline 8 & Pakistan & 2.4 & Cotton \\
\hline 9 & South Africa & 2.2 & Maize, soybean, cotton \\
\hline 10 & Uruguay & 1.1 & Soybean, maize \\
\hline 11 & Bolivia & 0.9 & Soybean \\
\hline 12 & Australia & 0.7 & Cotton, canola \\
\hline 13 & Philippines & 0.5 & Maize \\
\hline 14 & Myanmar & 0.3 & Cotton \\
\hline 15 & Burkina Faso & 0.3 & Cotton \\
\hline 16 & Spain & 0.1 & Maize \\
\hline 17 & Mexico & 0.1 & Cotton, soybean \\
\hline 18 & Columbia & $<0.1$ & Cotton \\
\hline 19 & Chile & $<0.1$ & Maize, soybean, canola \\
\hline 20 & Honduras & $<0.1$ & Maize \\
\hline 21 & Portugal & $<0.1$ & Maize \\
\hline 22 & Czech Republic & $<0.1$ & Maize, potato \\
\hline 23 & Poland & $<0.1$ & Maize \\
\hline 24 & Egypt & $<0.1$ & Maize \\
\hline 25 & Slovakia & $<0.1$ & Maize \\
\hline 26 & Costa Rica & $<0.1$ & Cotton, soybean \\
\hline 27 & Romania & $<0.1$ & Maize \\
\hline 28 & Sweden & $<0.1$ & Potato \\
\hline 29 & Germany & $<0.1$ & Potato \\
\hline
\end{tabular}

Source: James, C. 2010. http://isaaa.org/resources/publications/briefs/42/executivesummary/default.asp 


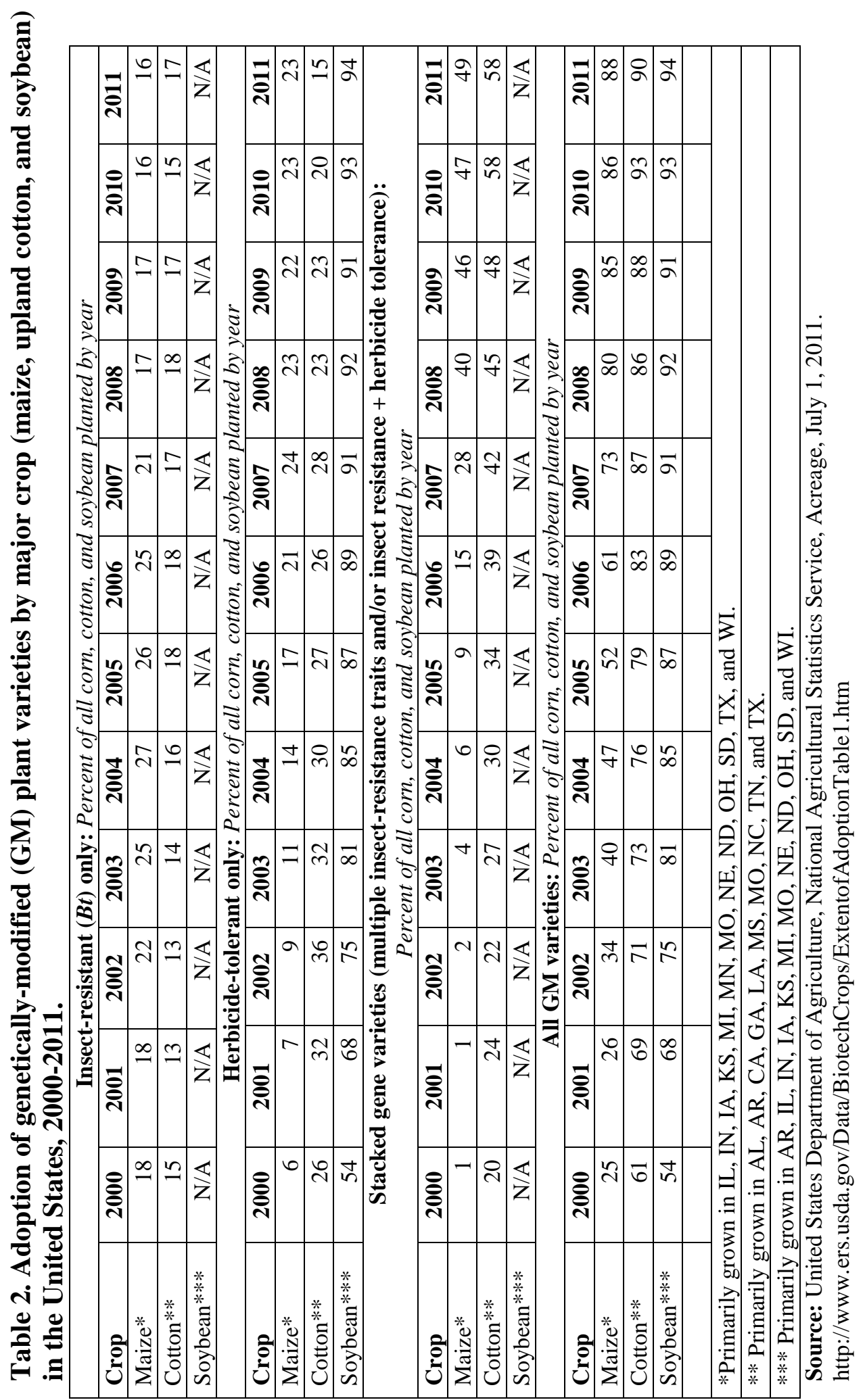




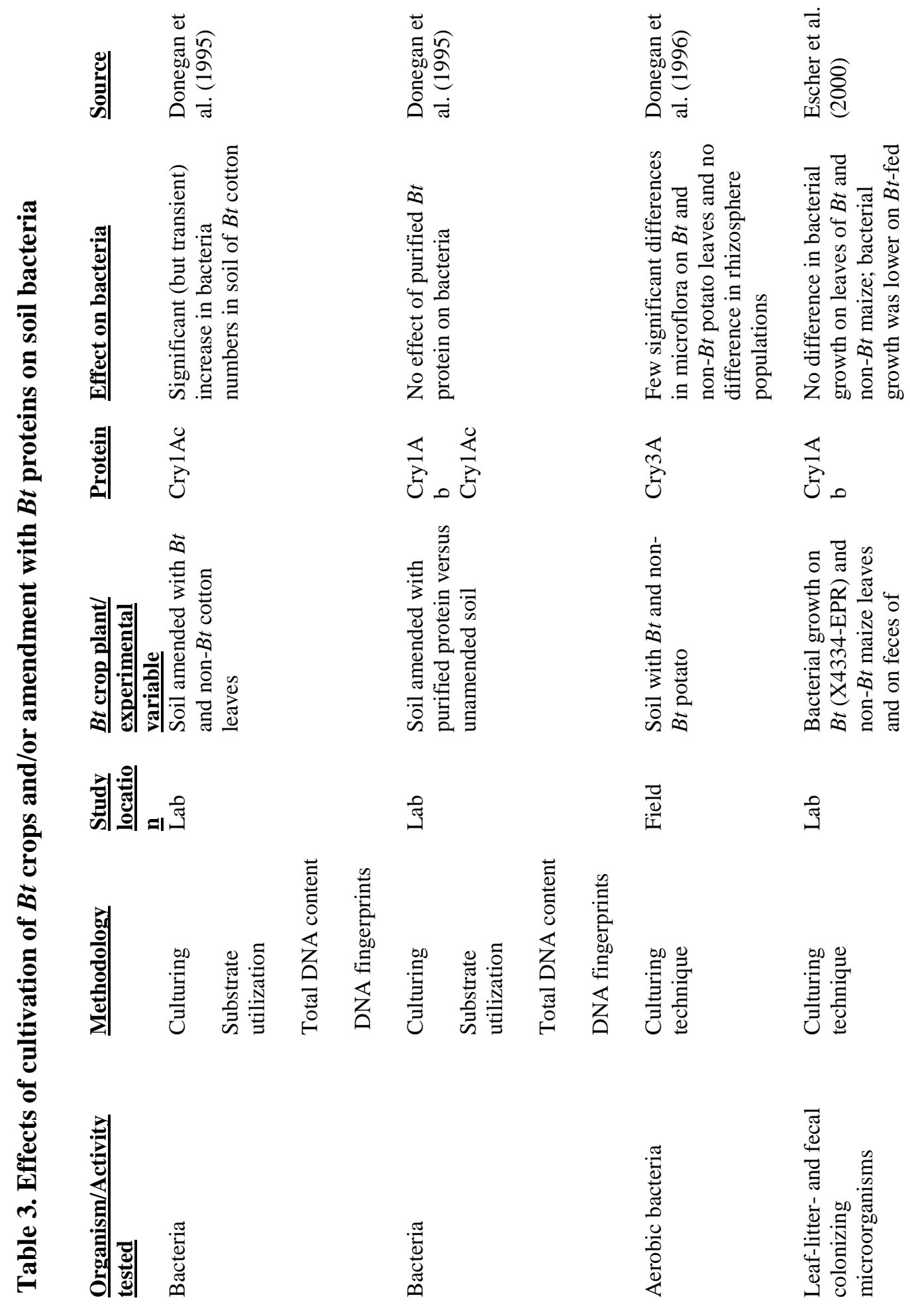




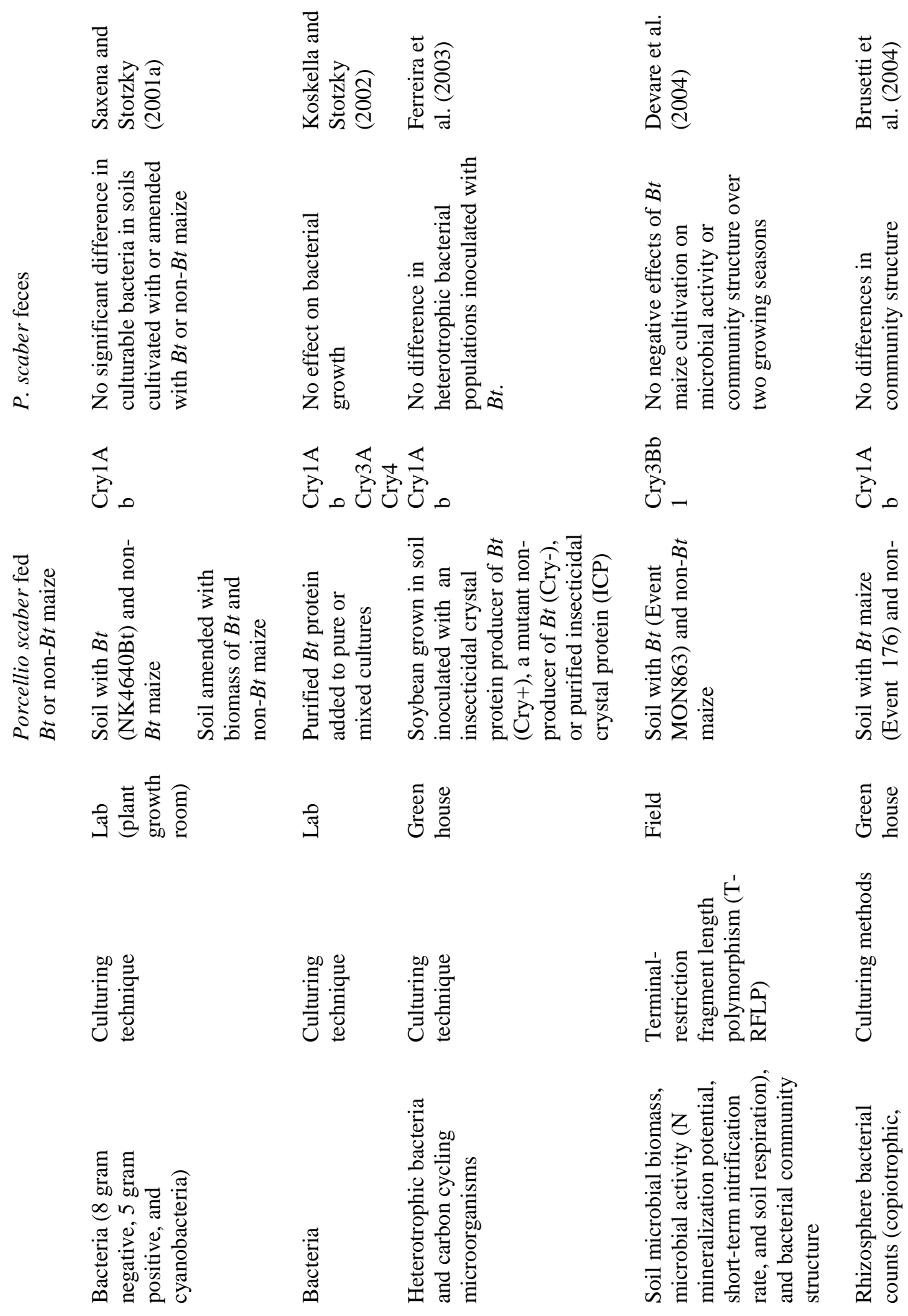




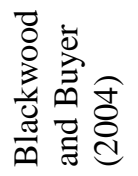

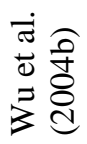

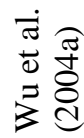
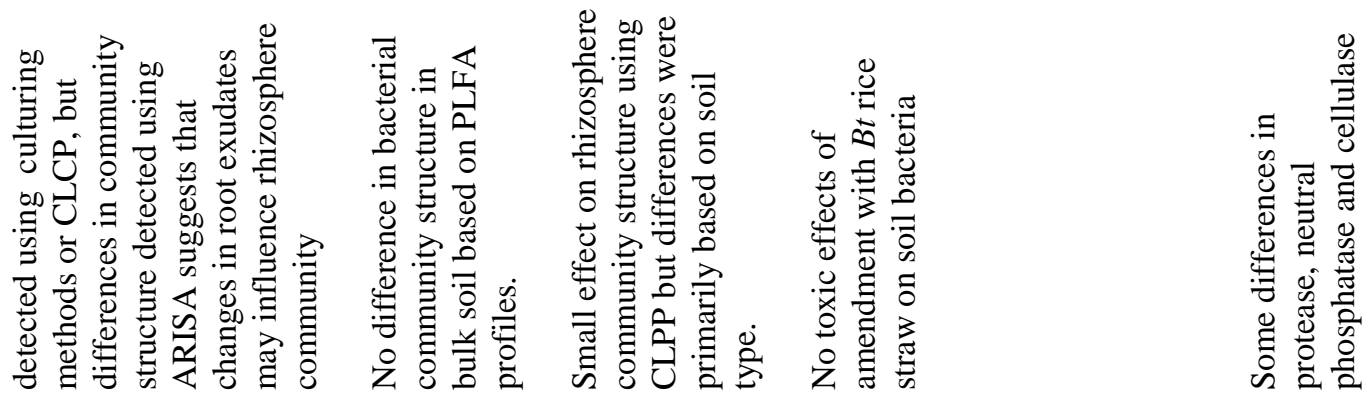

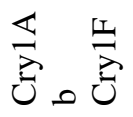

通。

¿
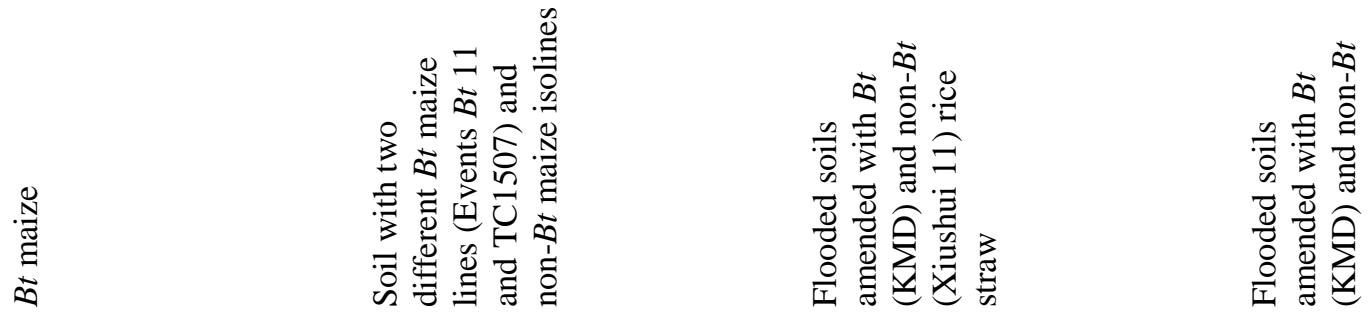

离

త్

తี

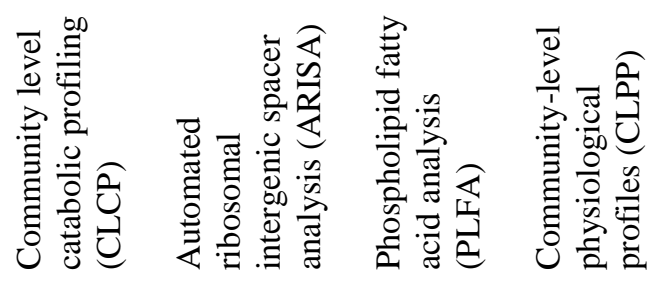

Uू.
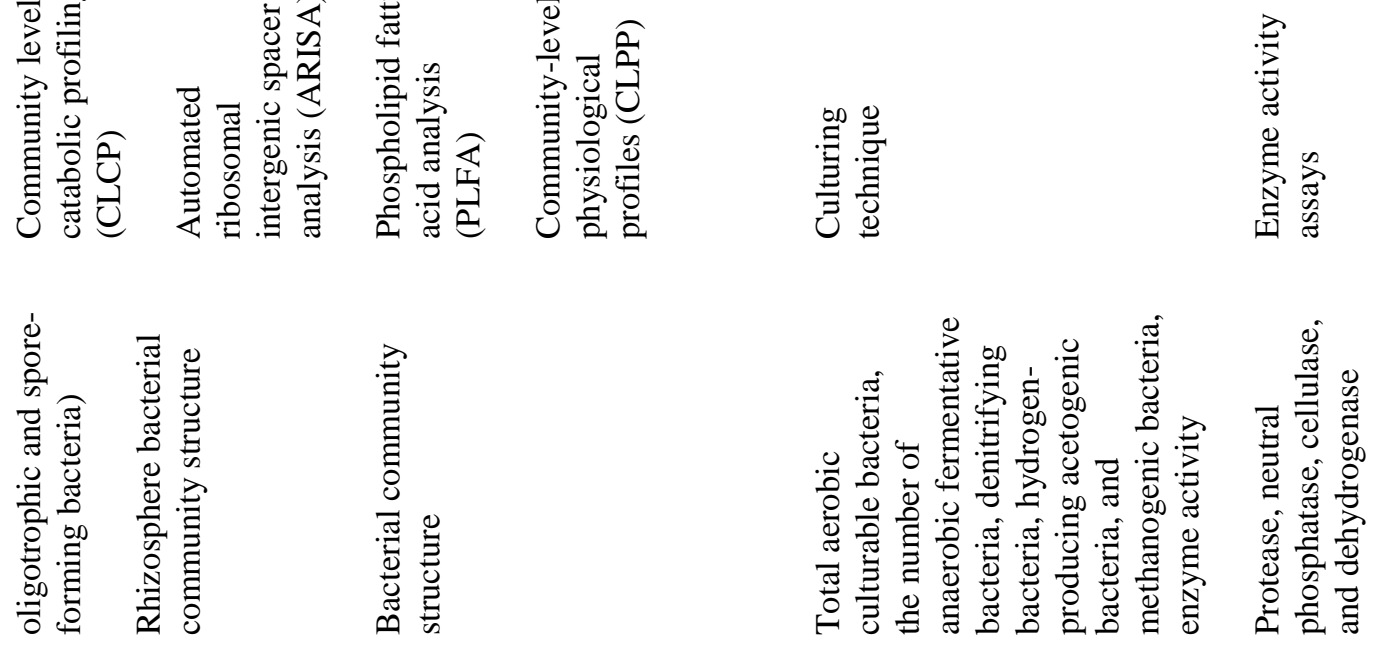

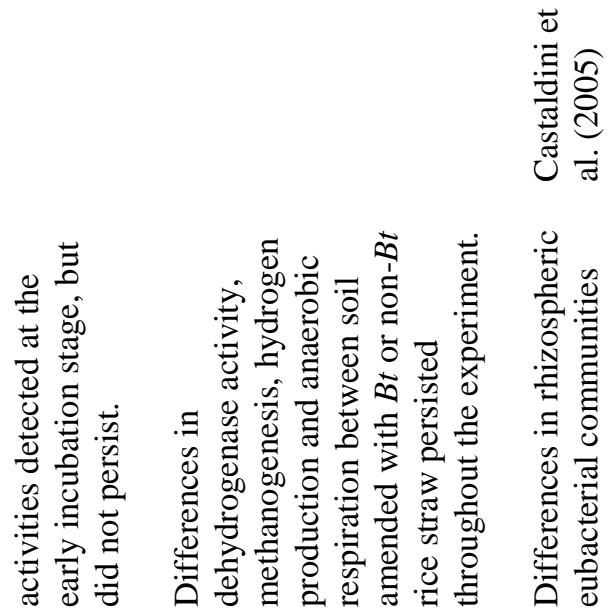

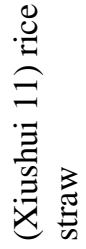
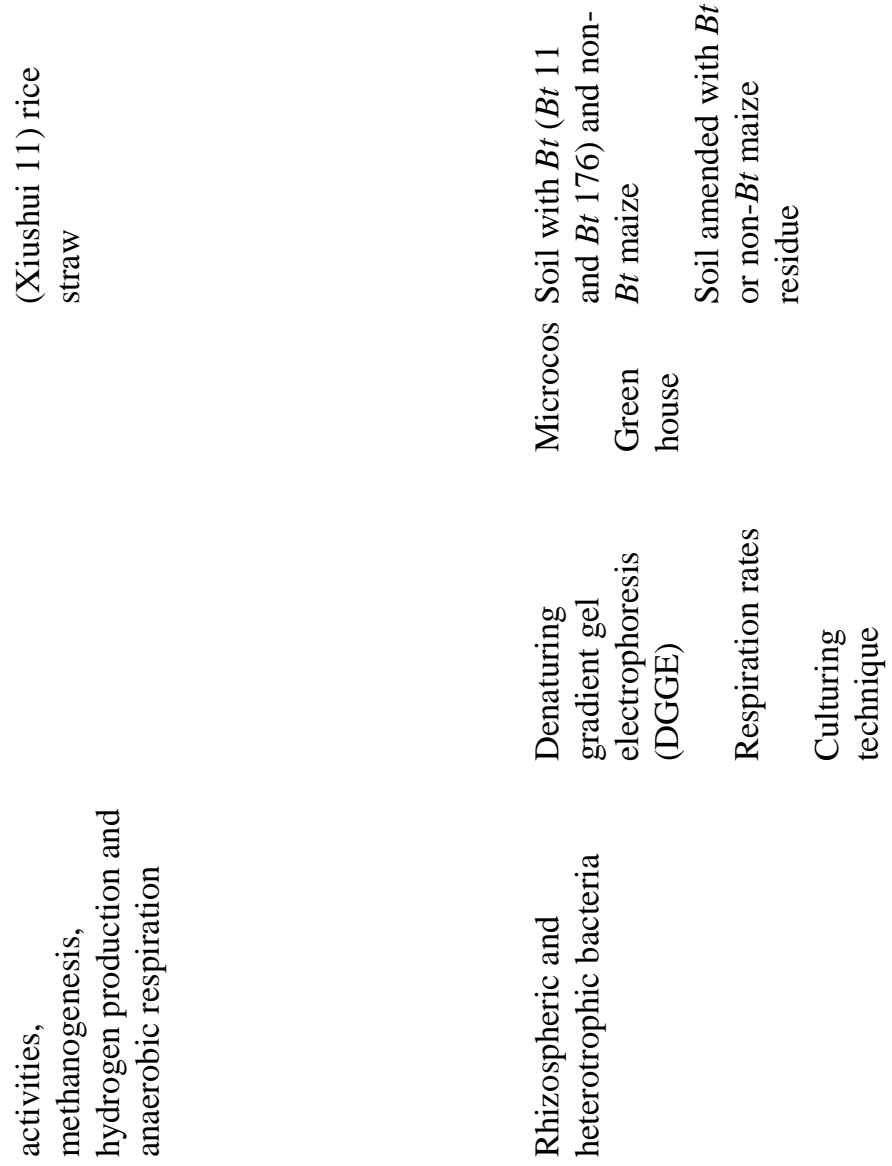
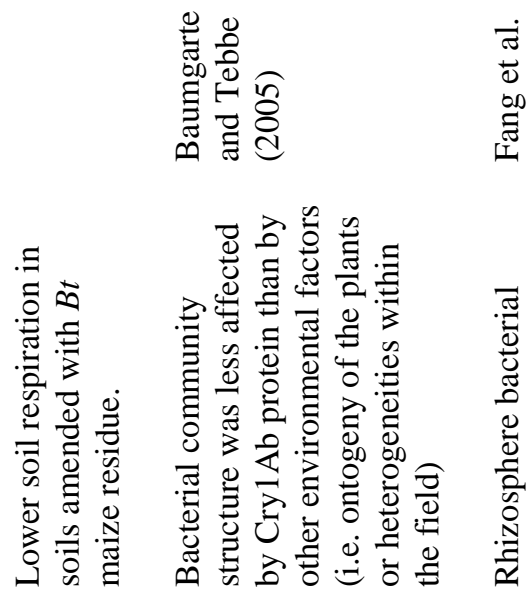

$\stackrel{\Xi}{\Xi}$

$\underset{\Xi}{\mathbb{U}}$

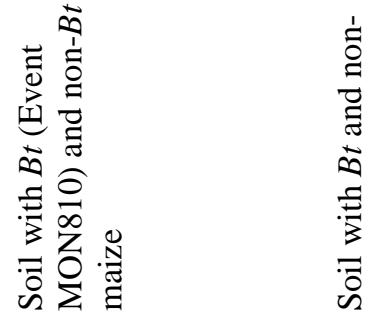

$\frac{7}{2}$

ญี
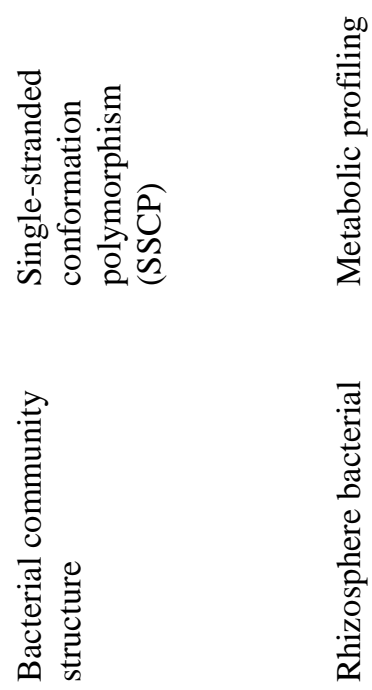

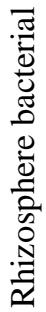




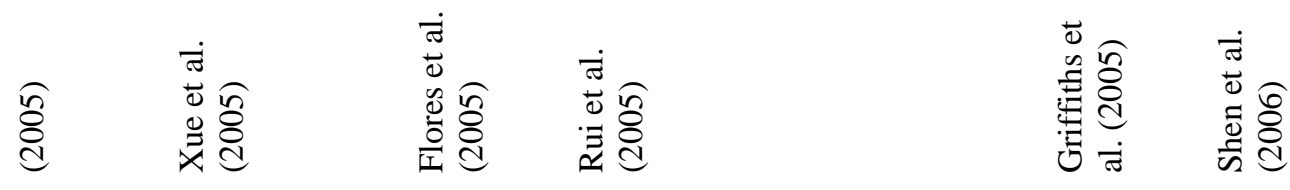

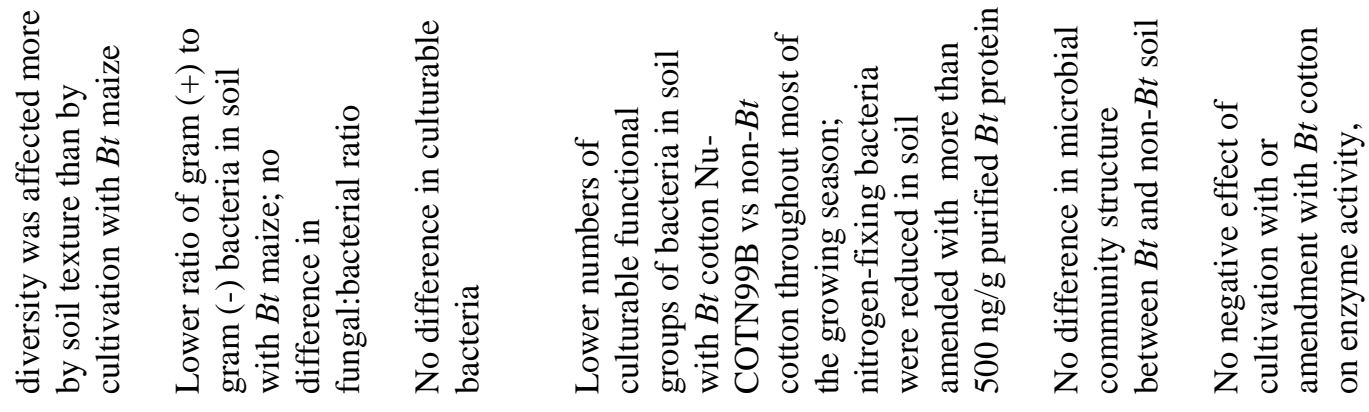

0 妏。

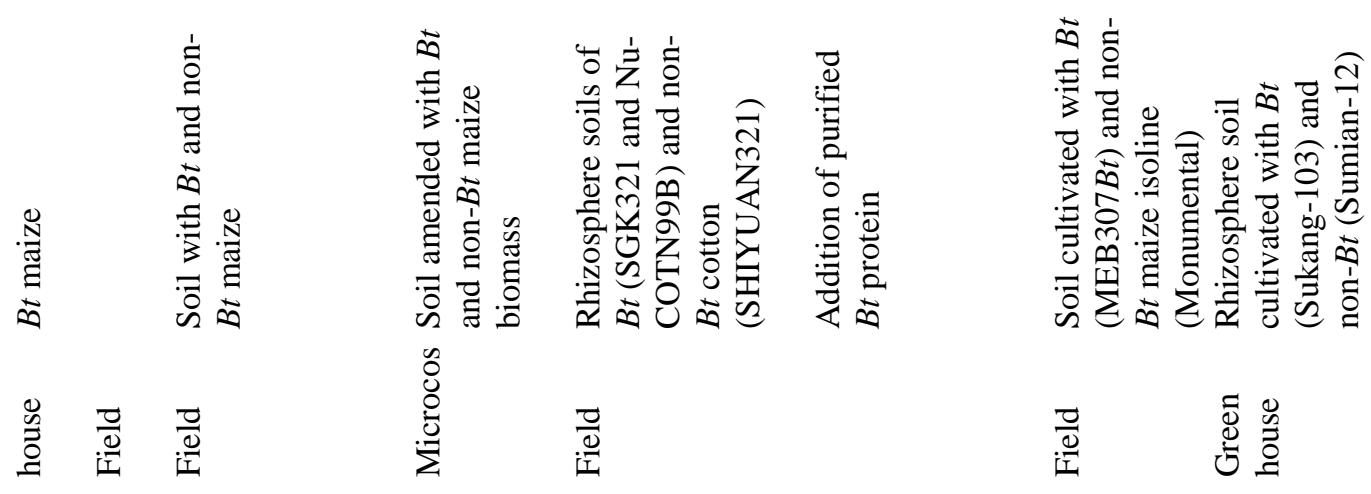

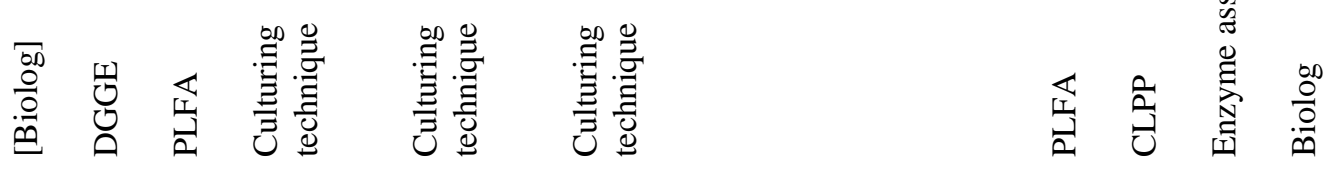

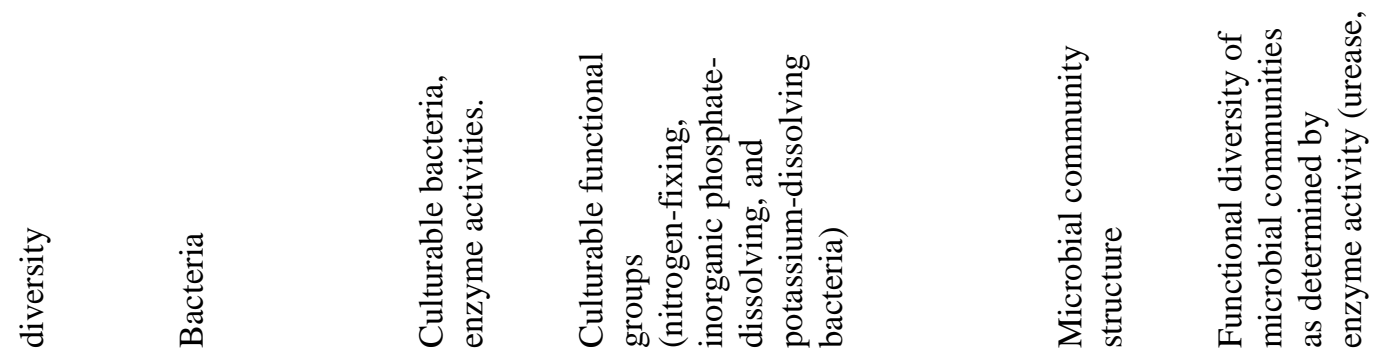



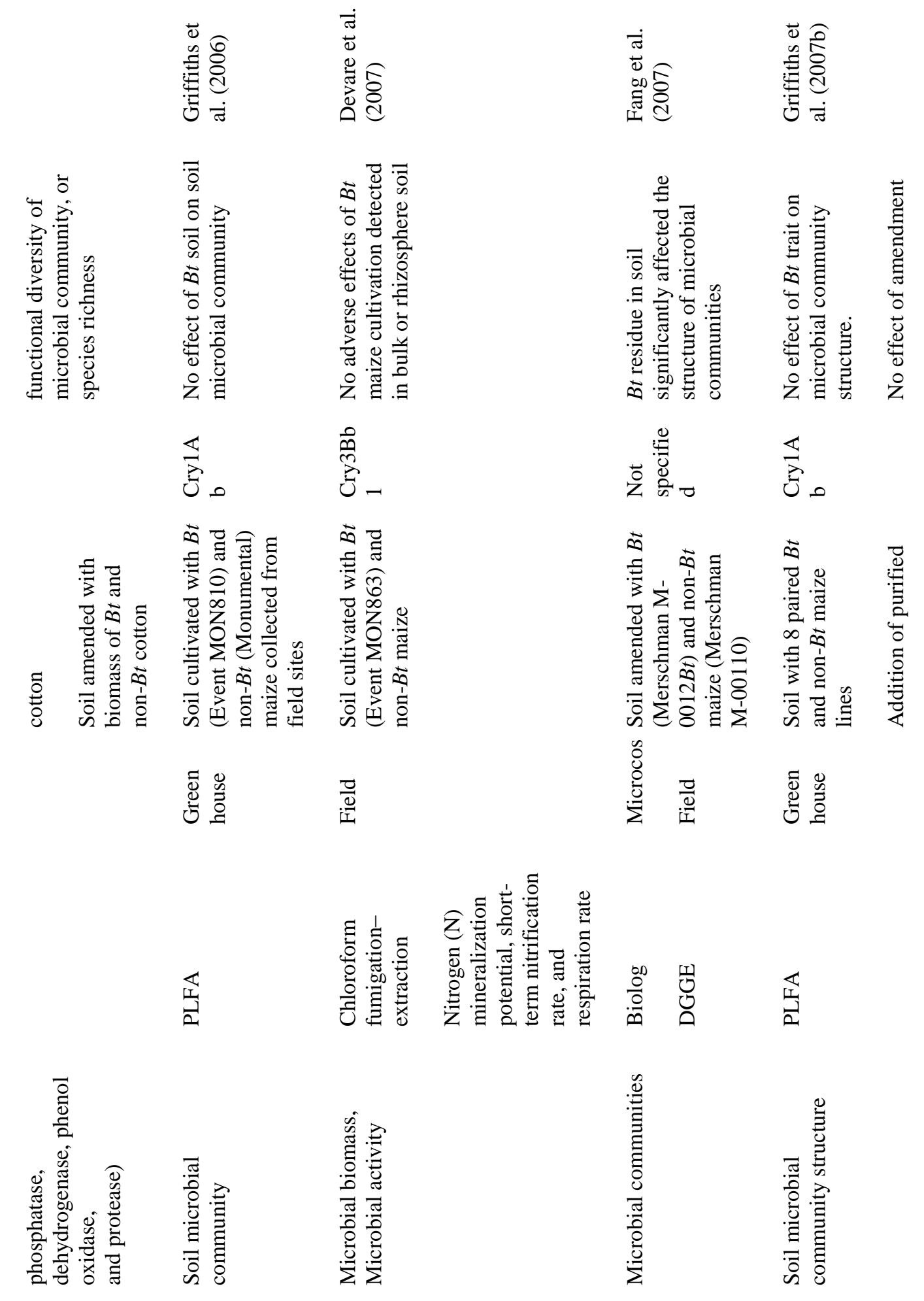

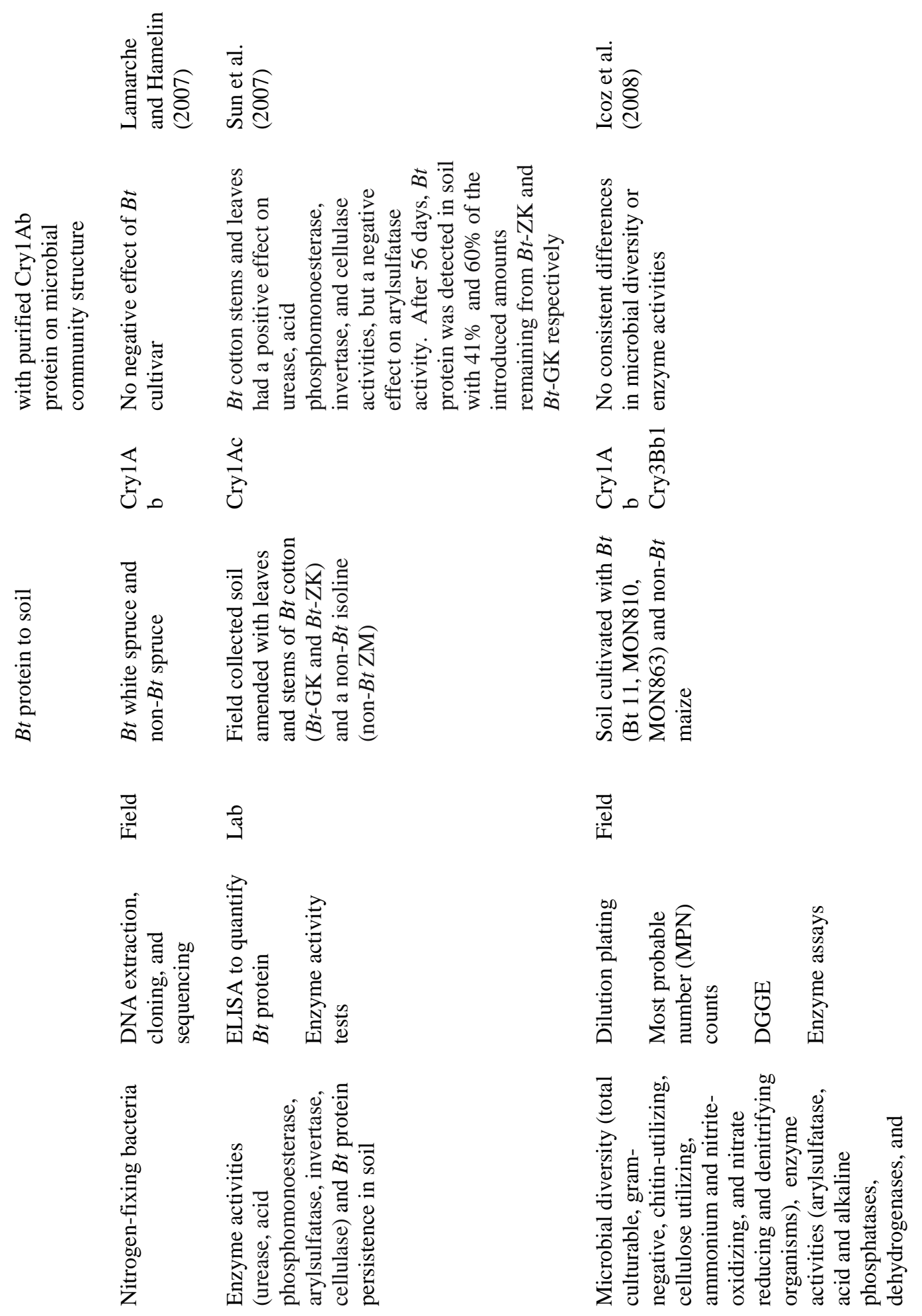

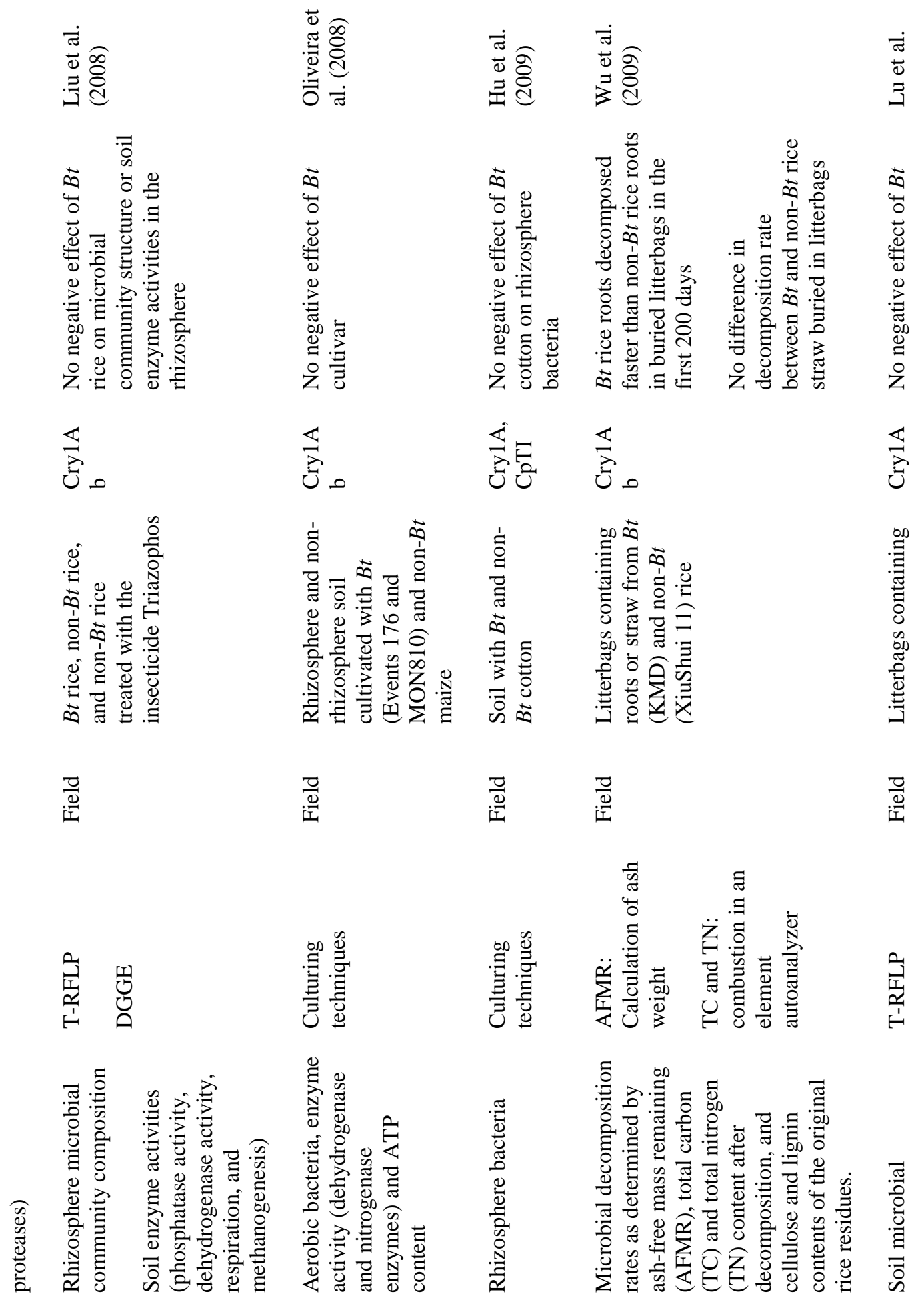

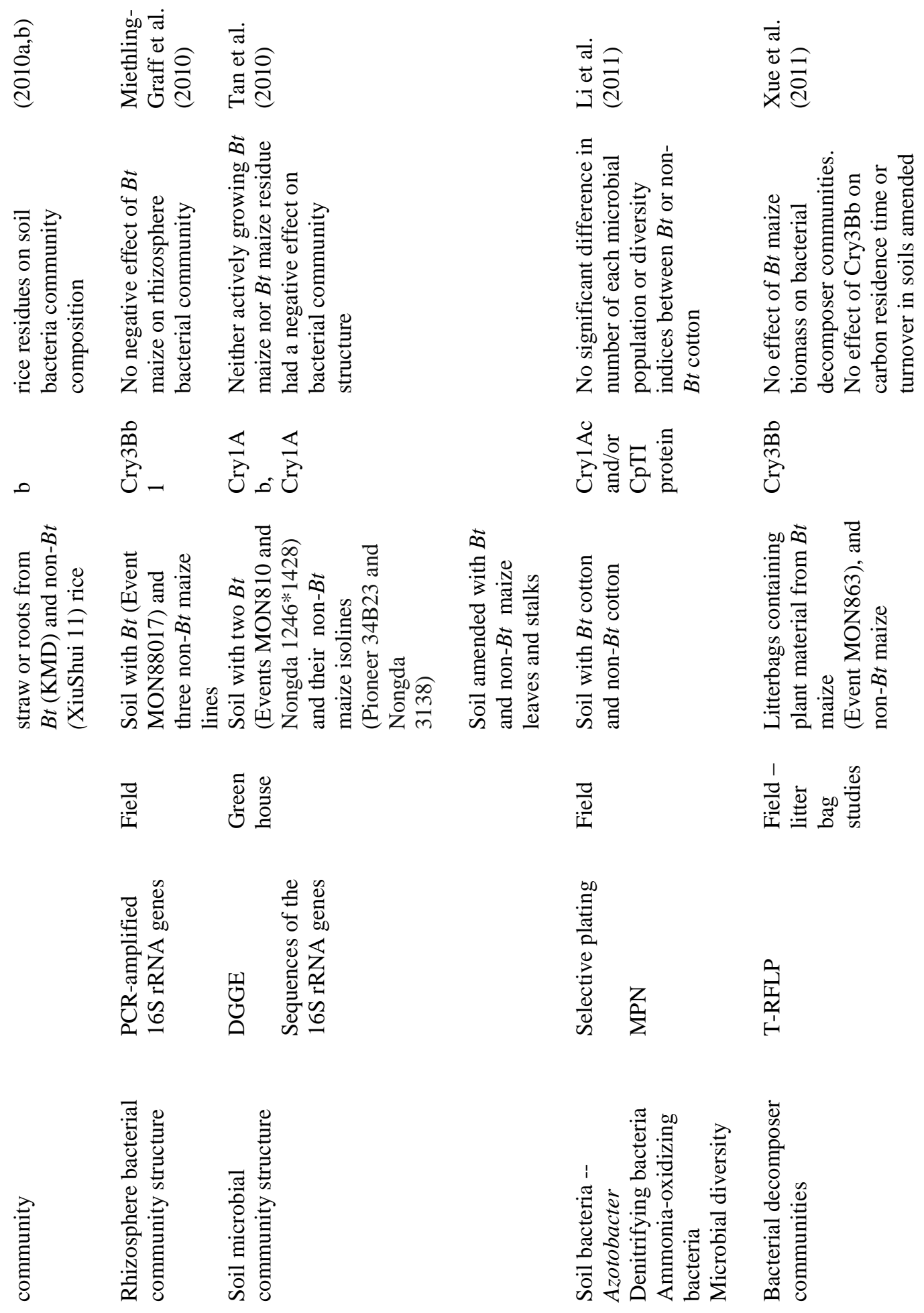


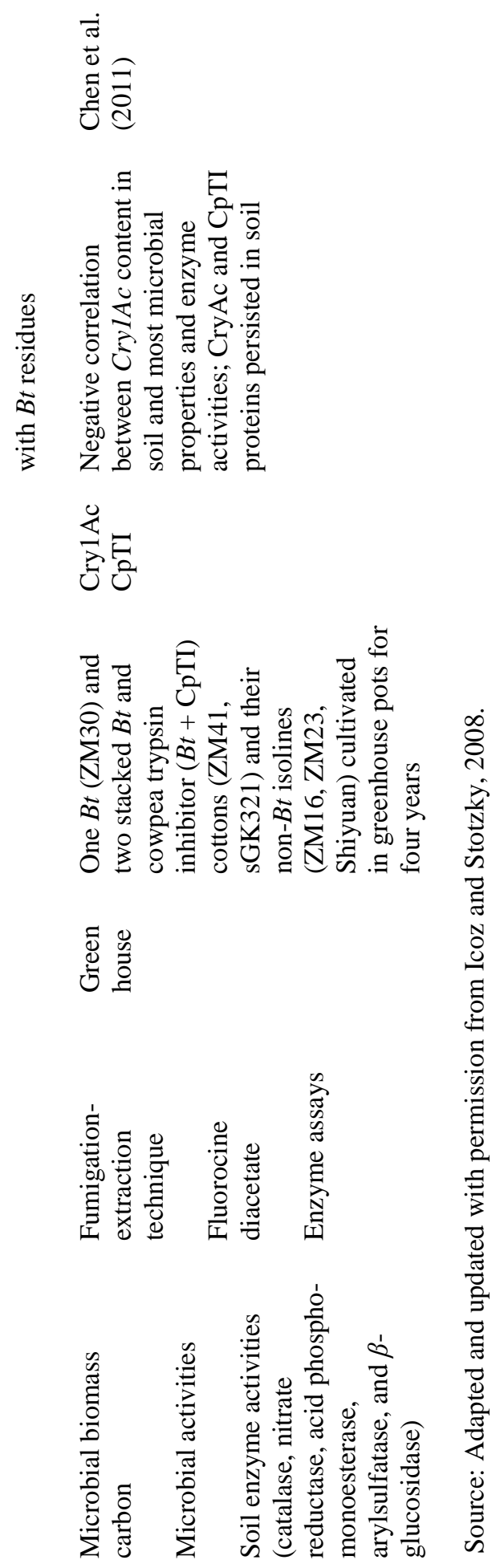




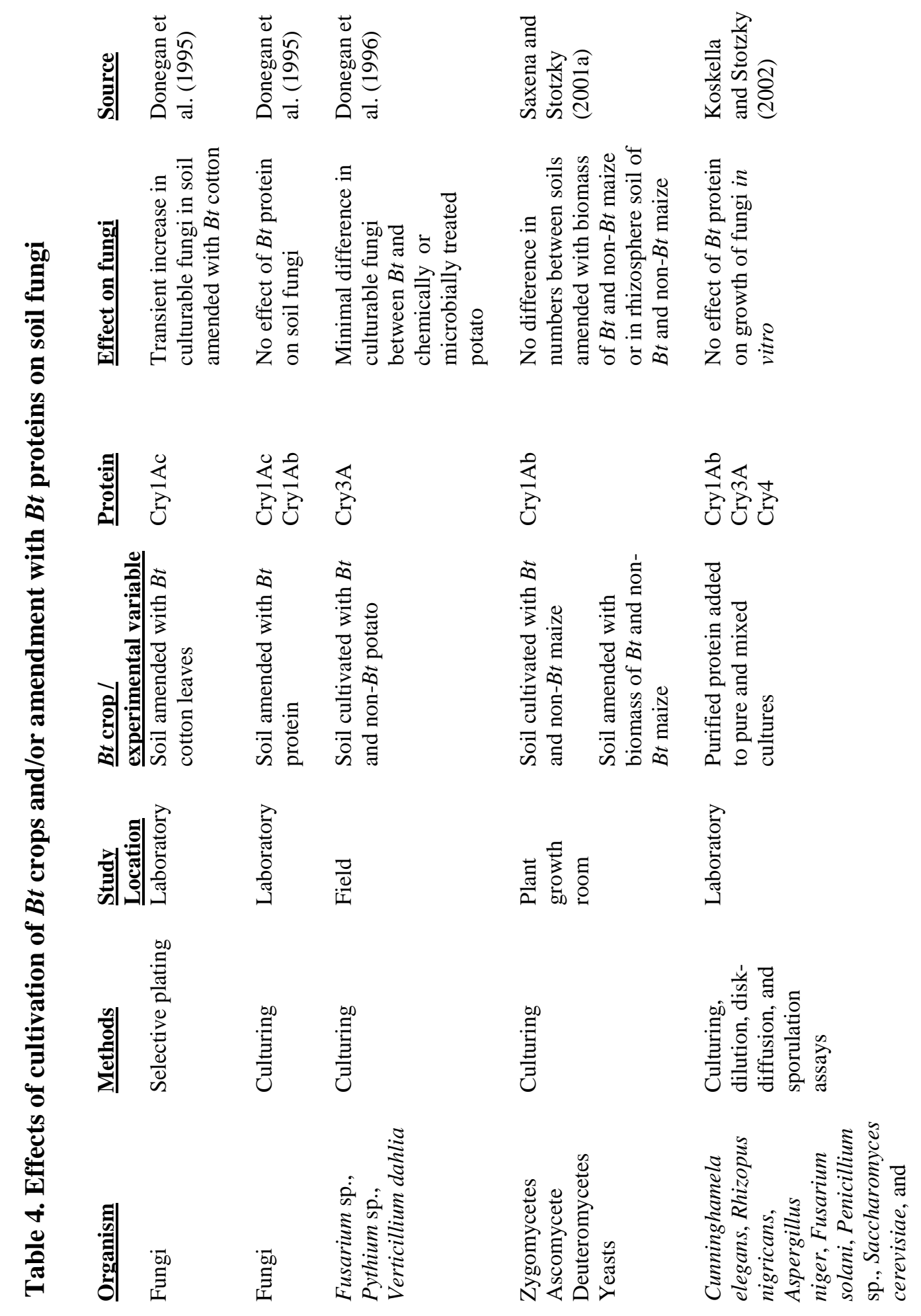




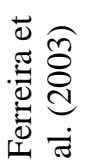
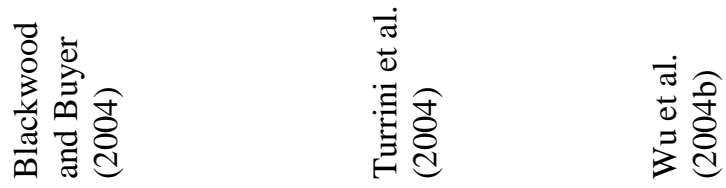

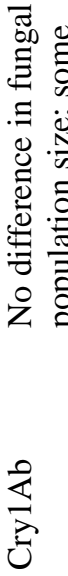

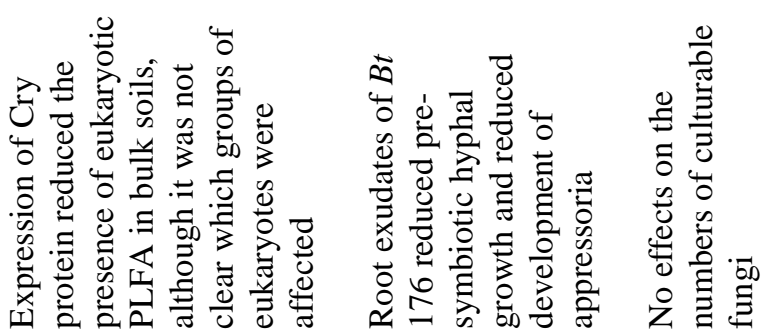

造至

主

导
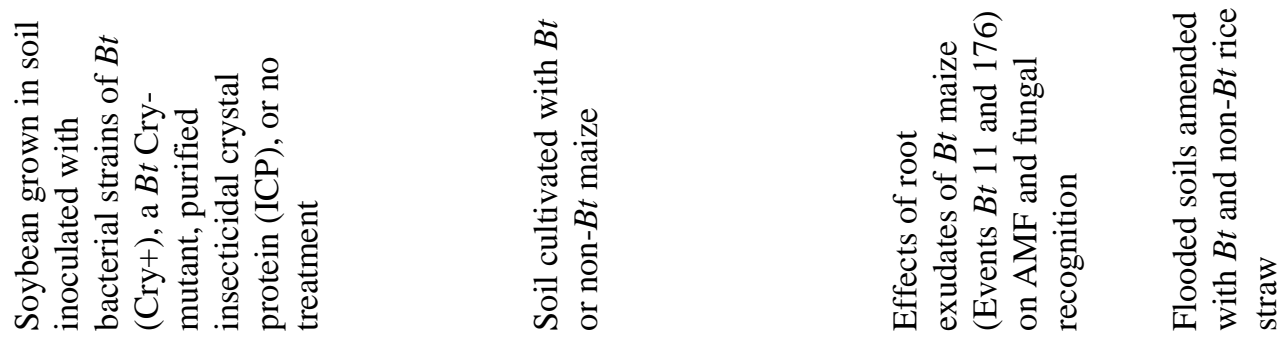

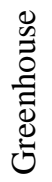

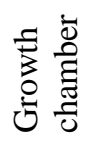
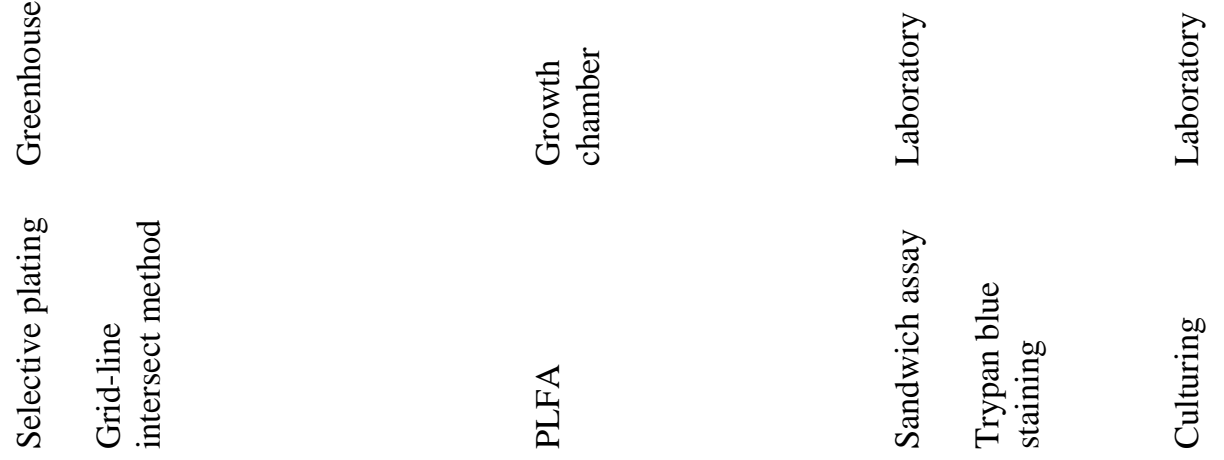

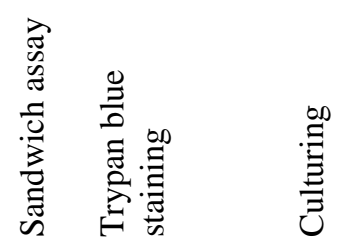

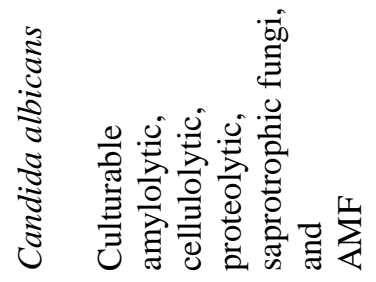

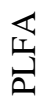




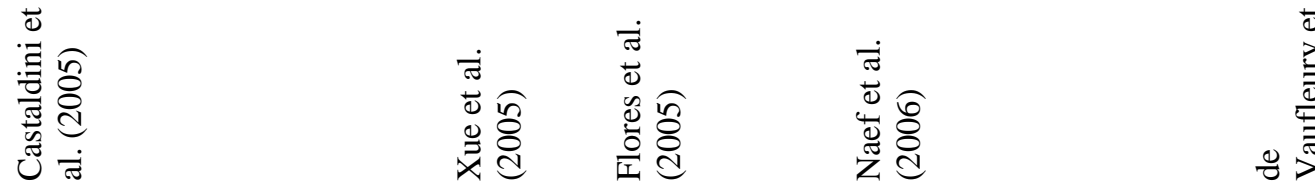

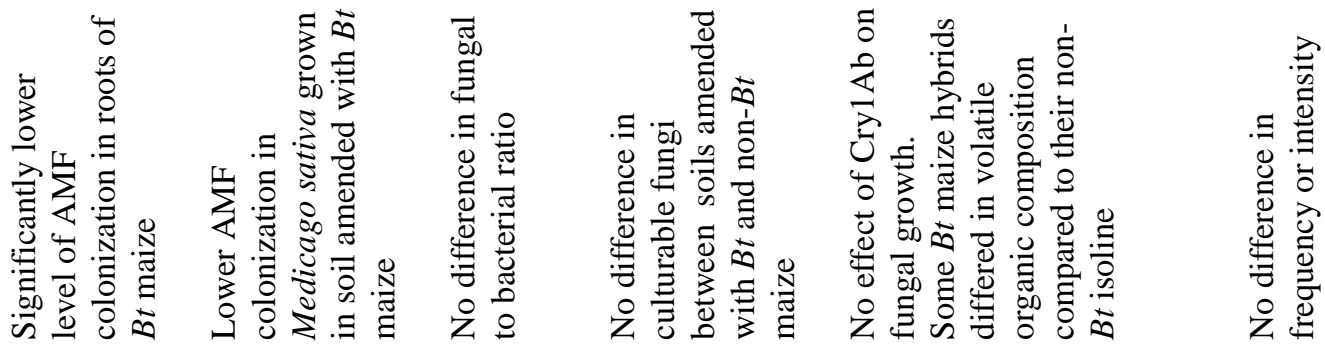

这

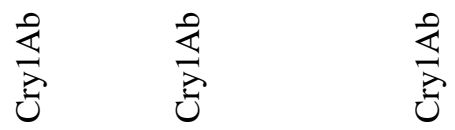

这
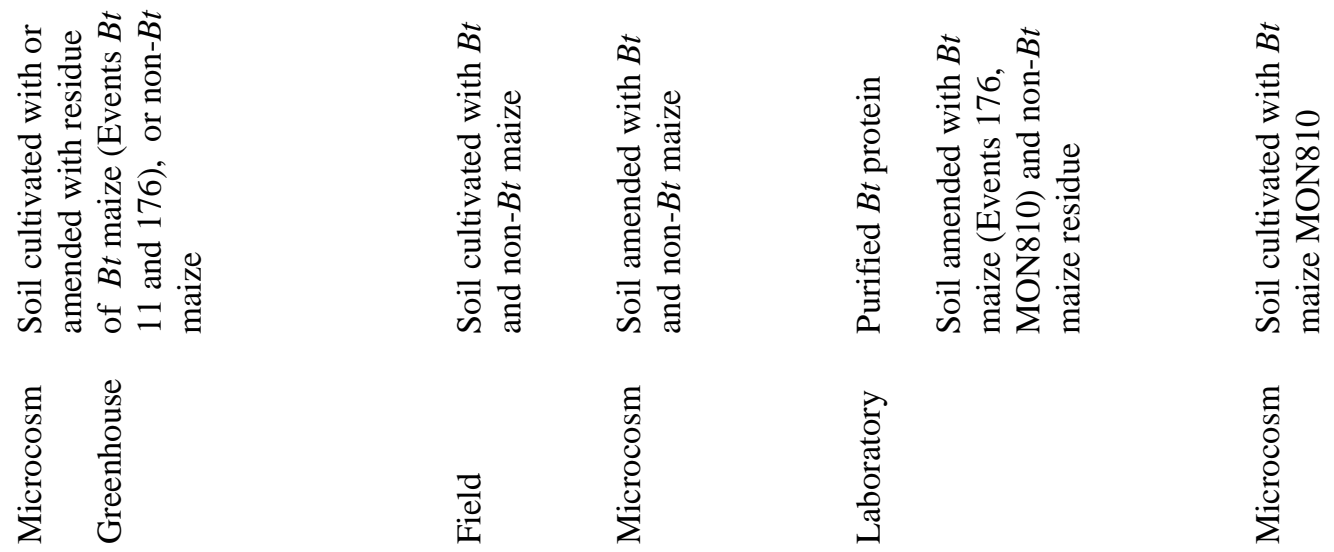

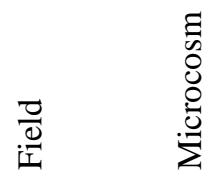
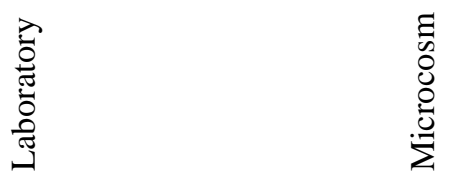

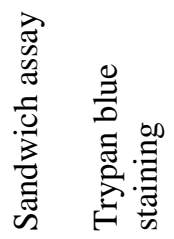

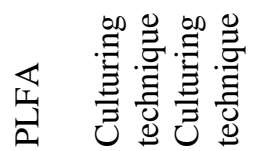

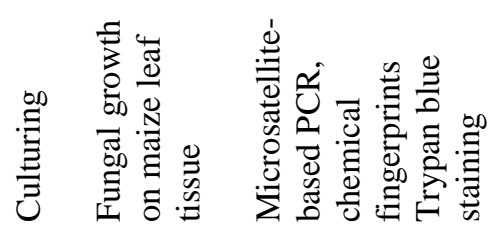

章

要 覀

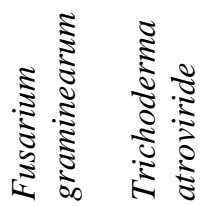

$\sum$ 

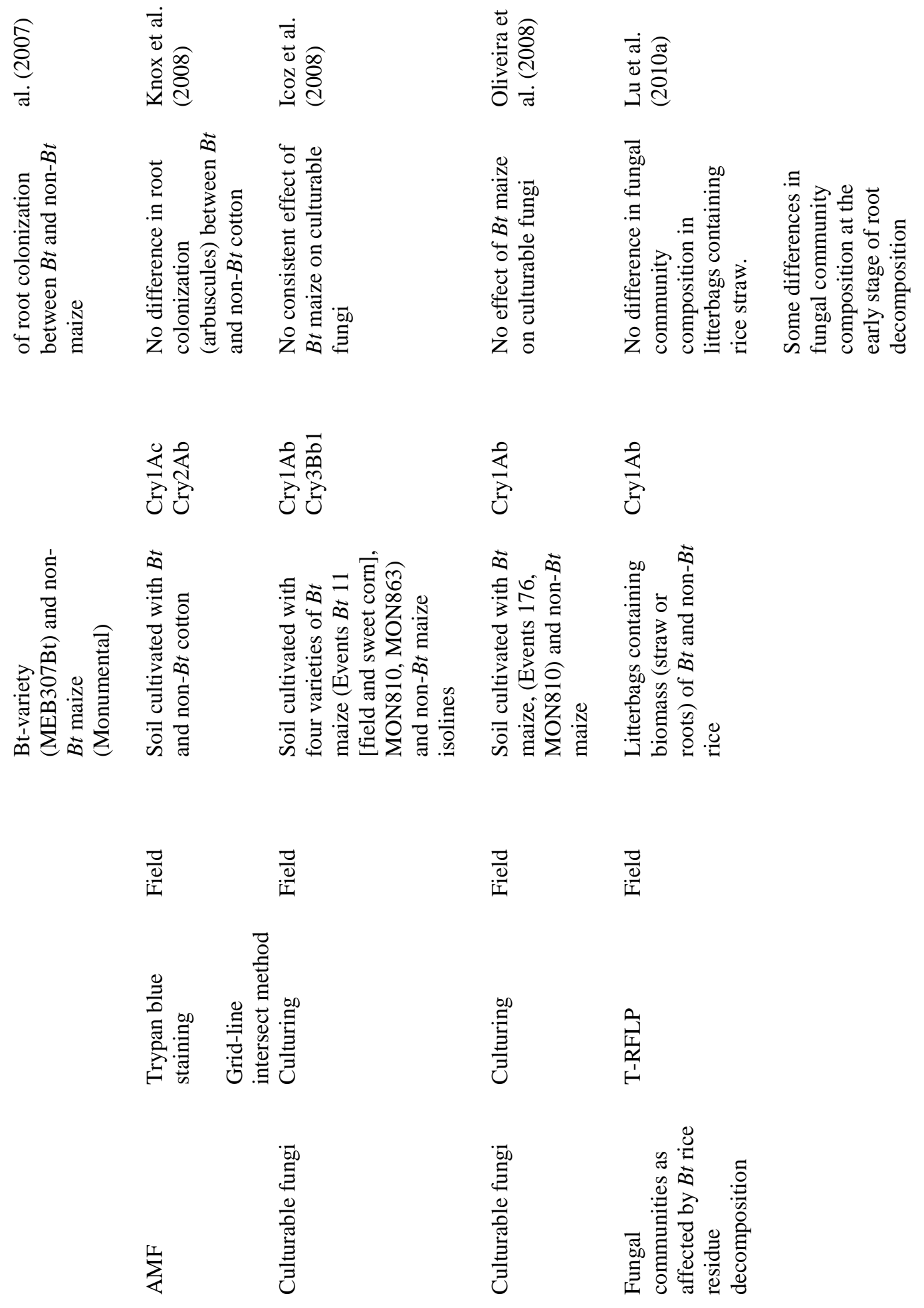


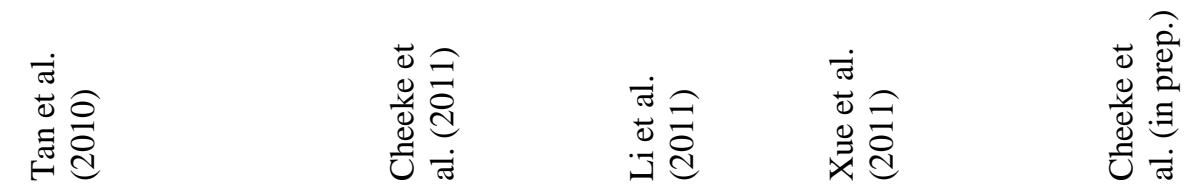

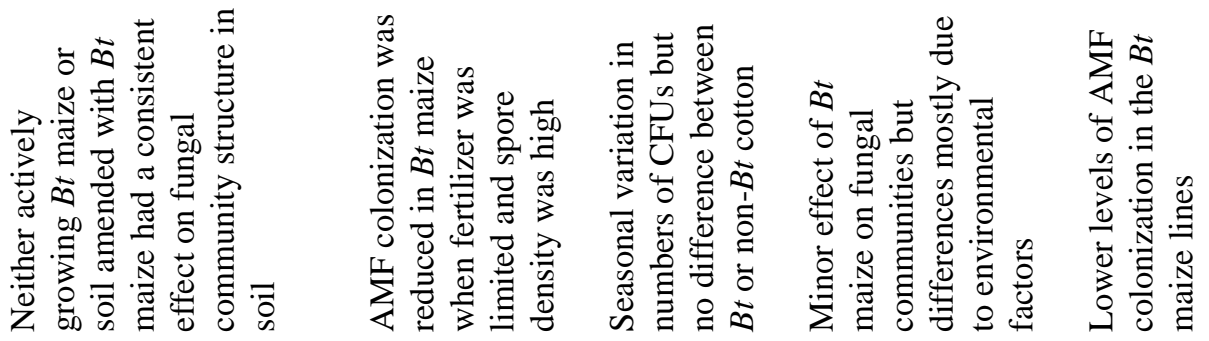

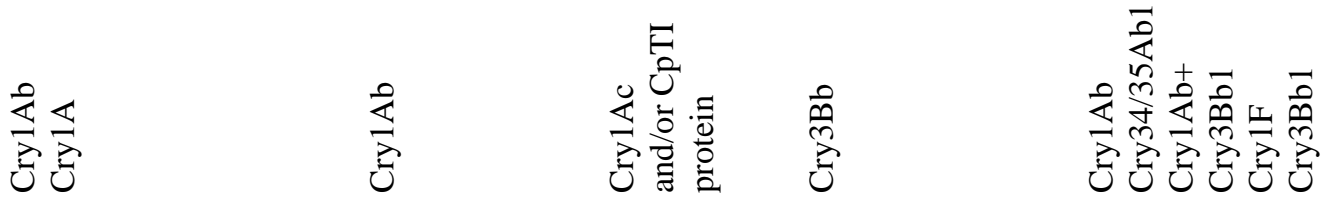

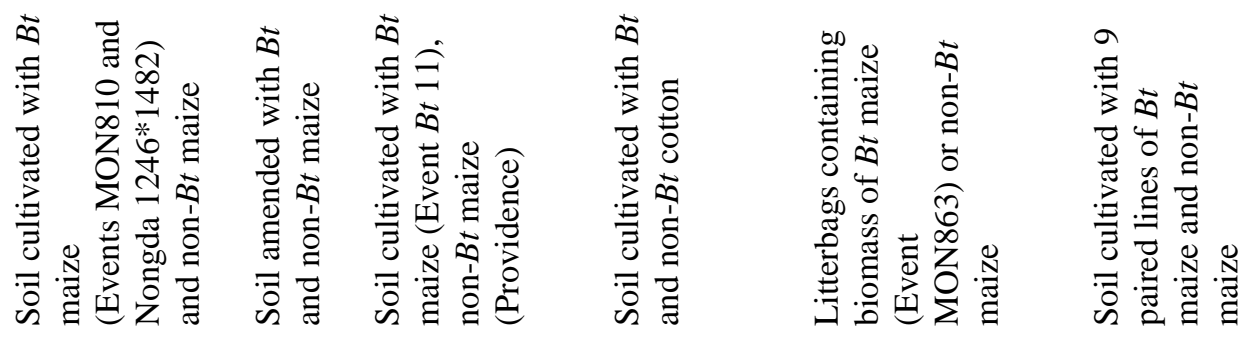

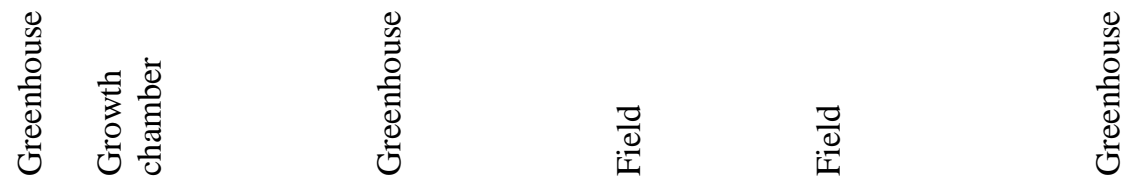

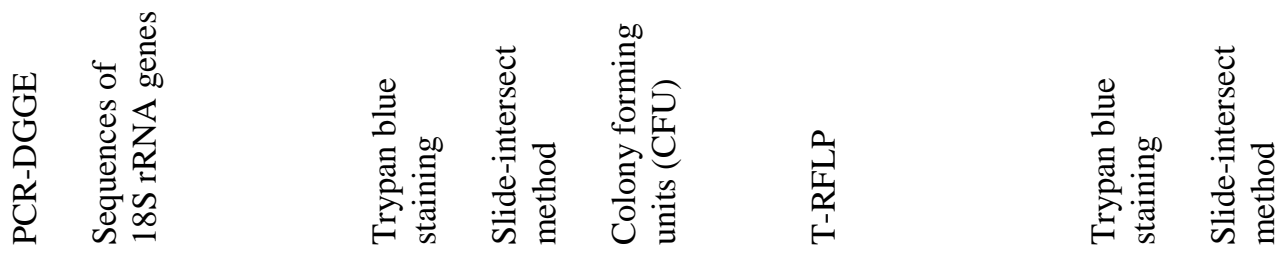

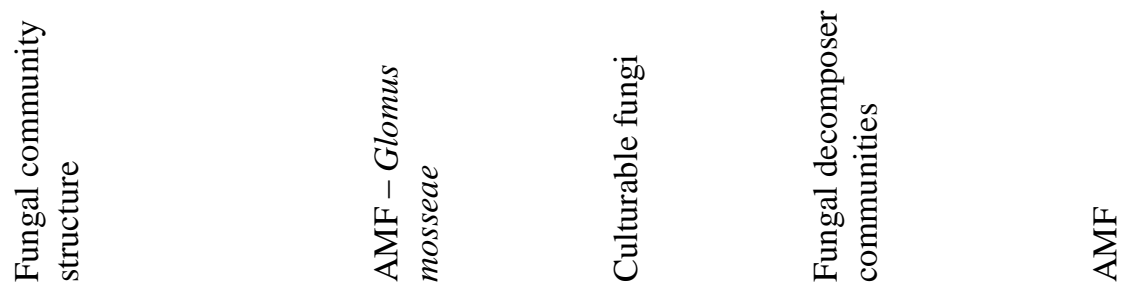




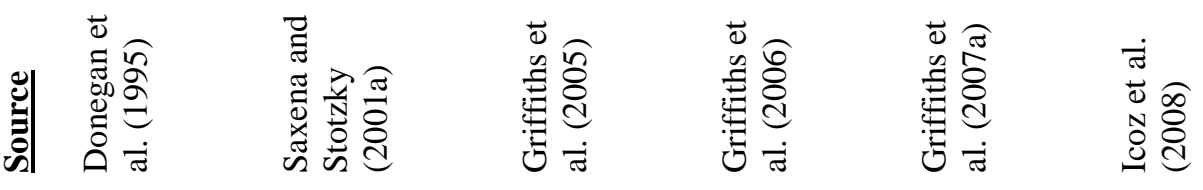

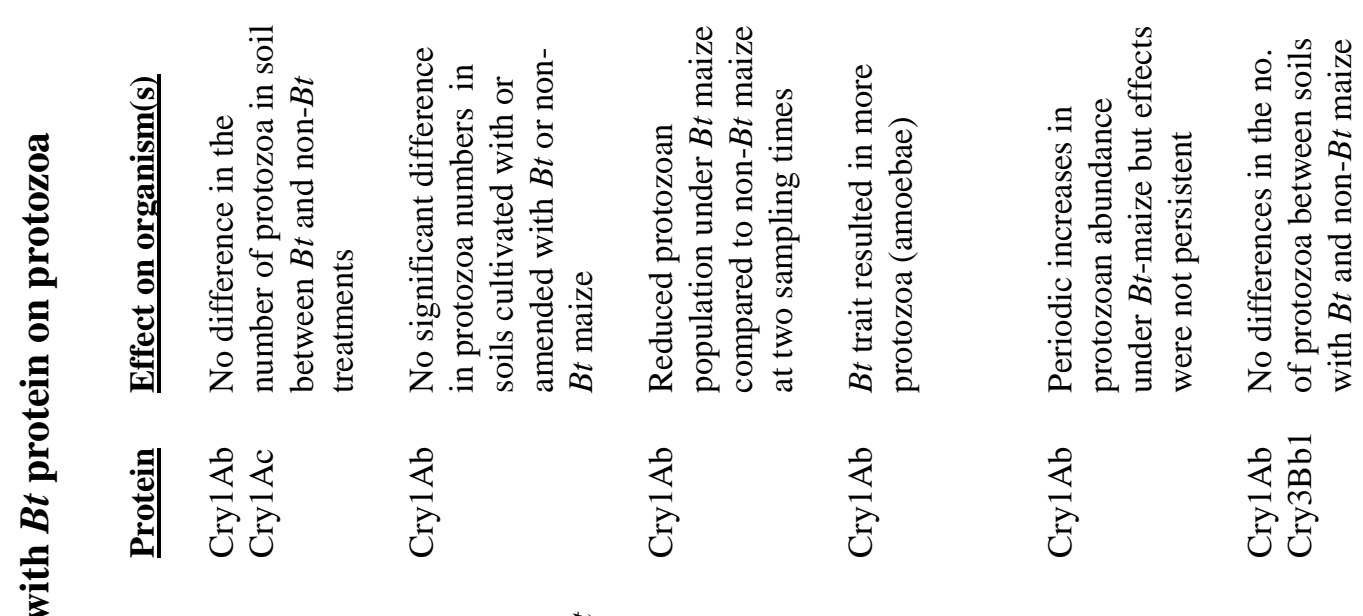

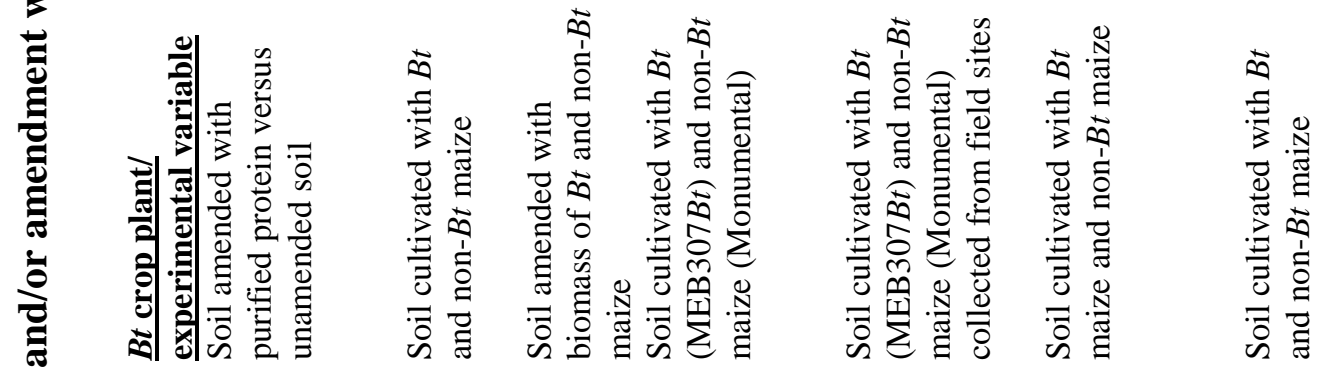

苞
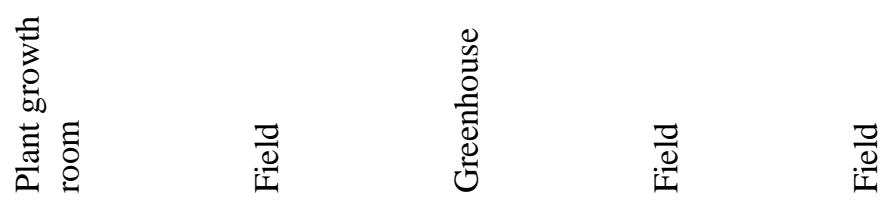

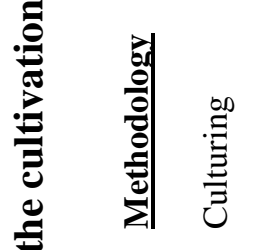

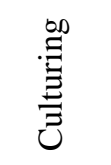

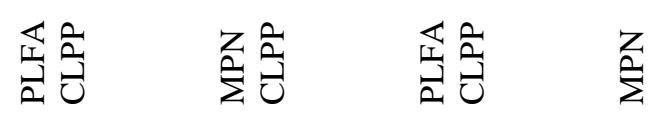
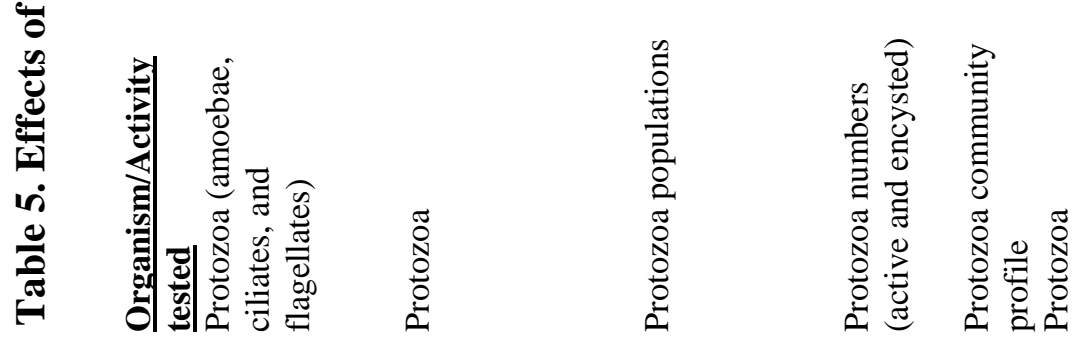

\begin{tabular}{l}
\multirow{2}{0}{} \\
0 \\
0 \\
0
\end{tabular} 


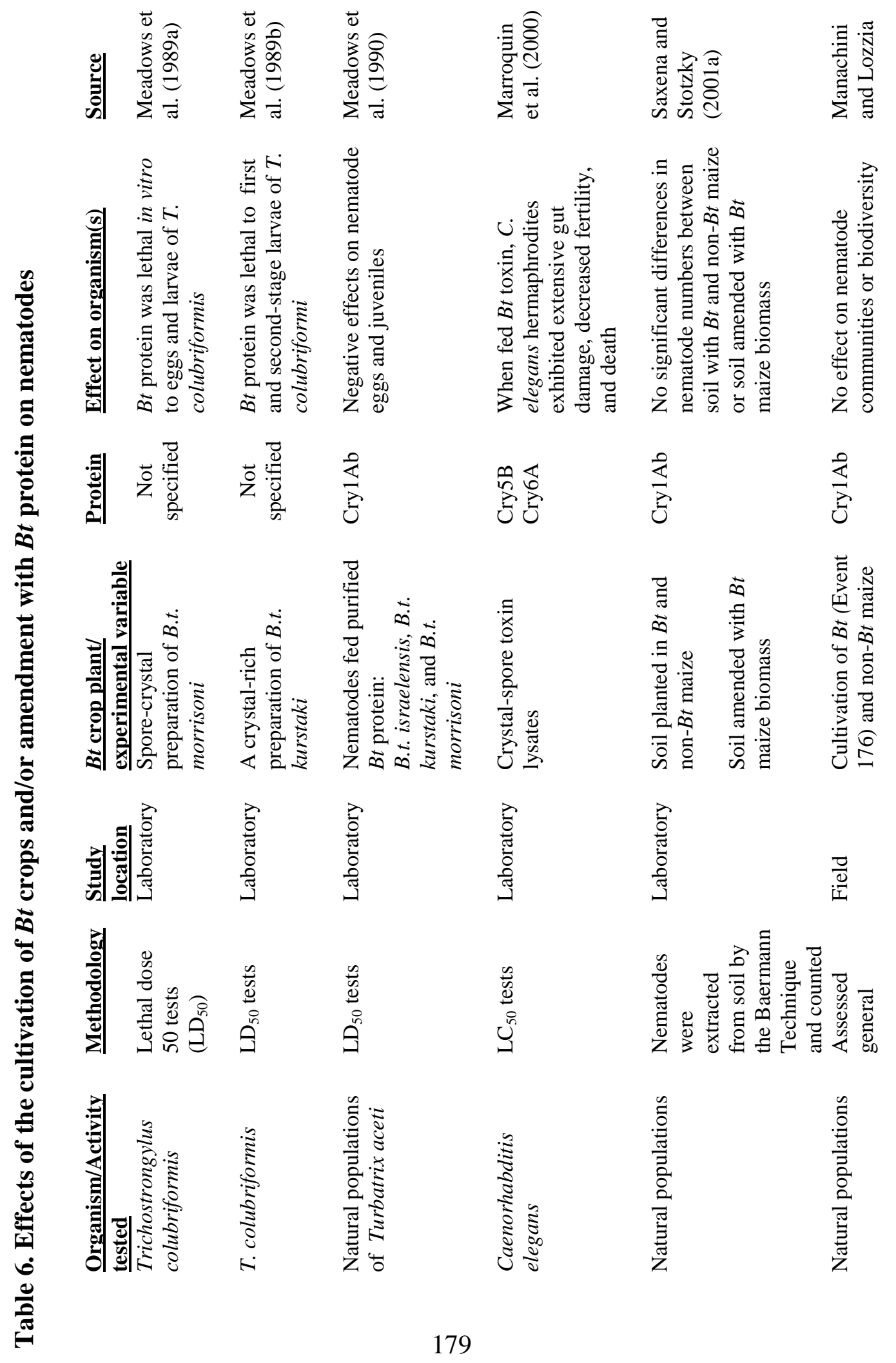



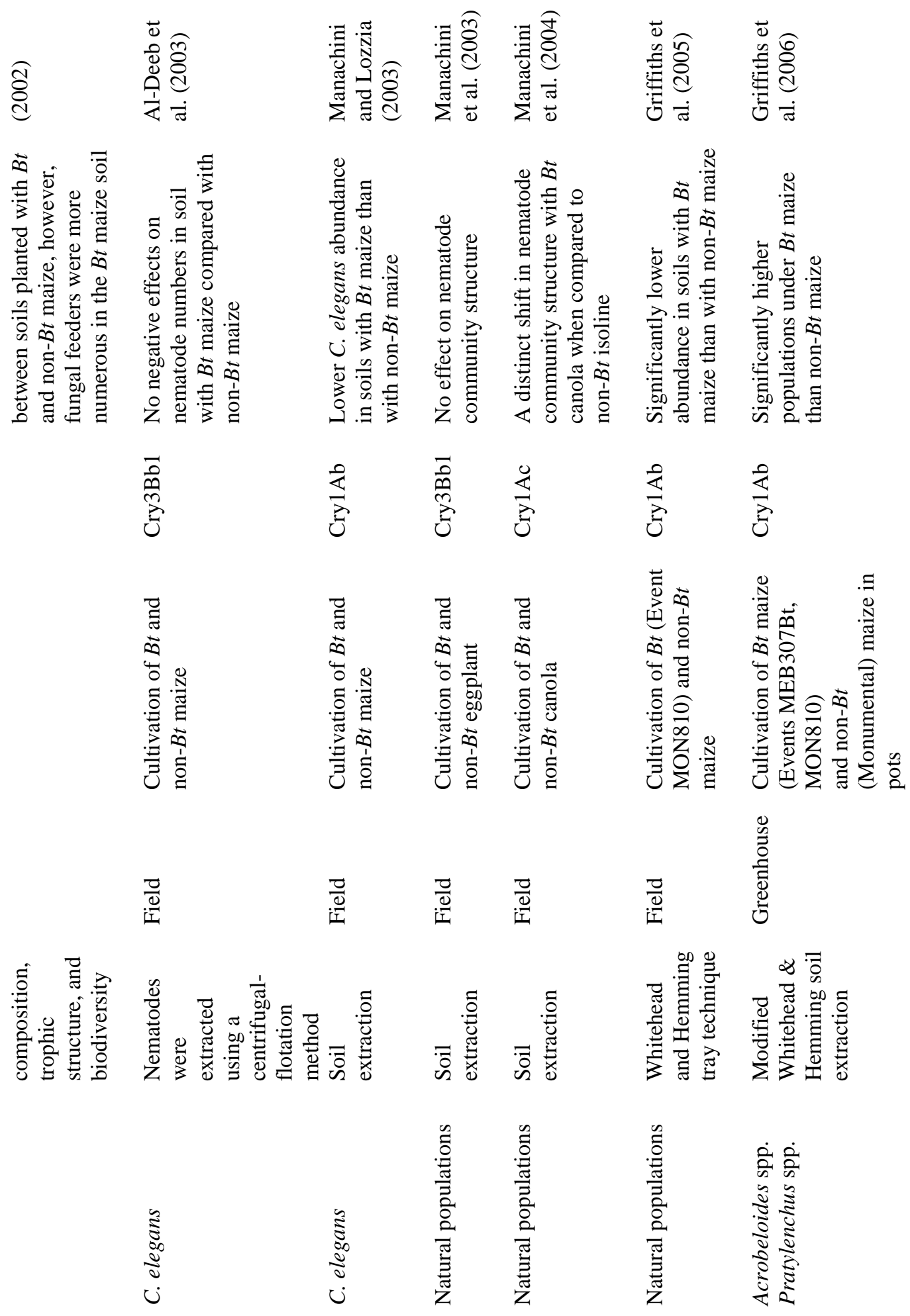


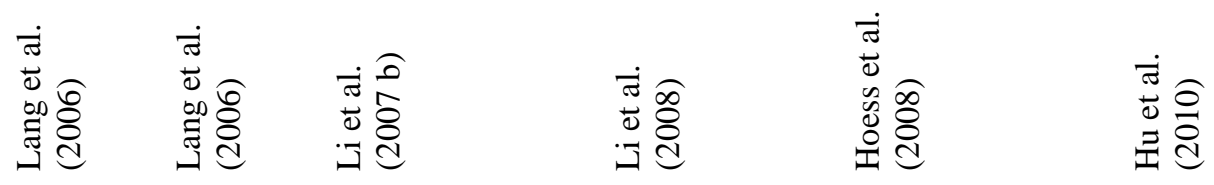

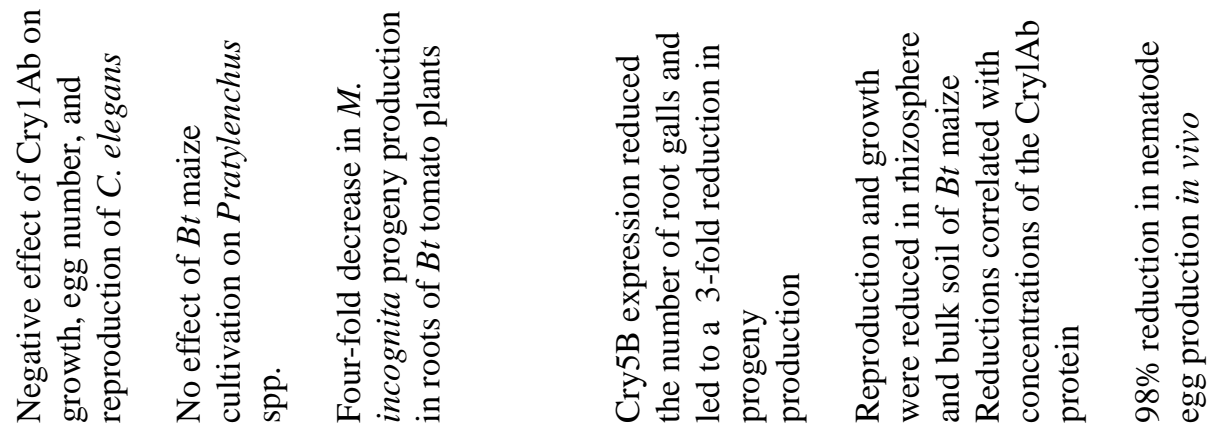

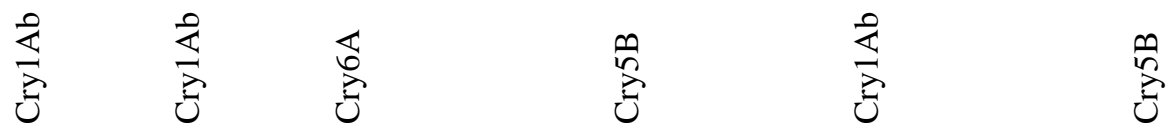

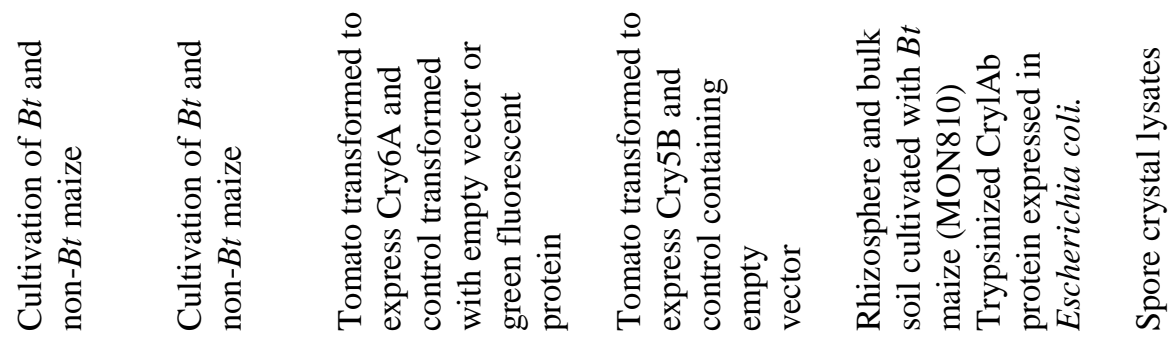

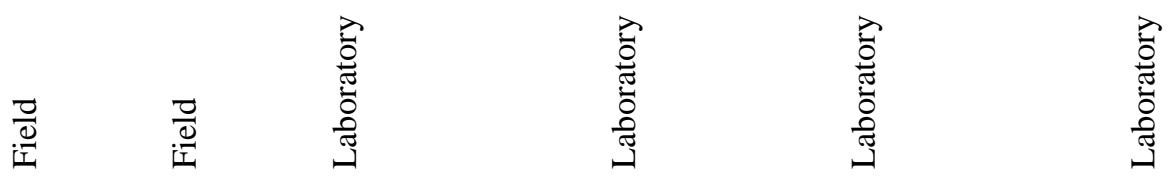
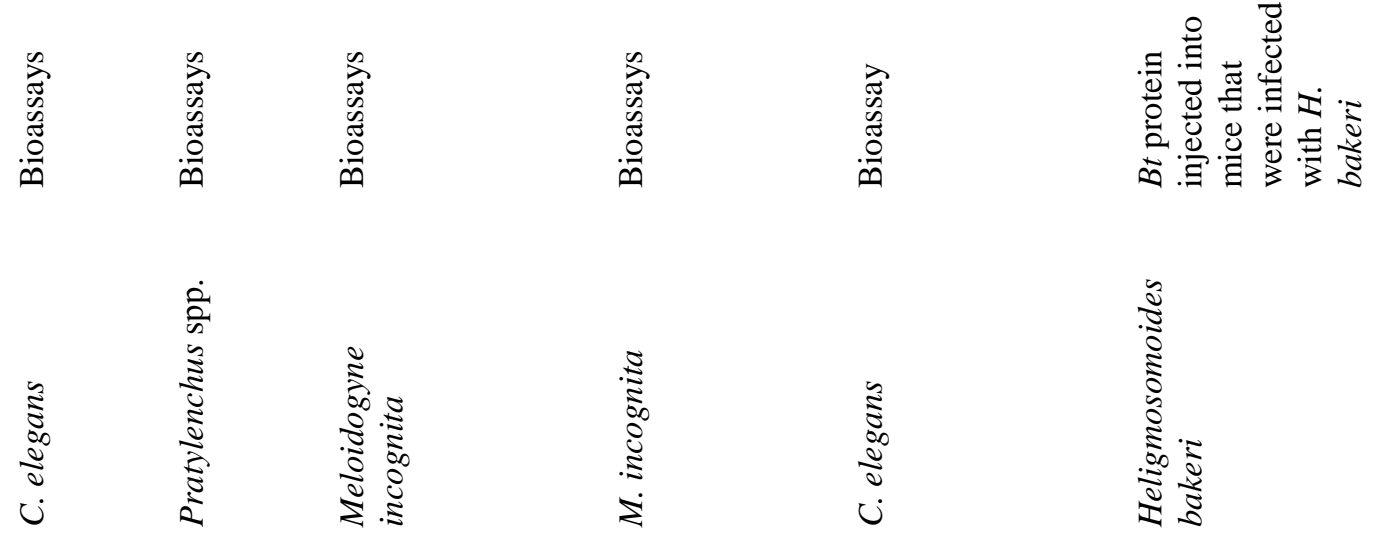


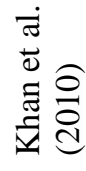

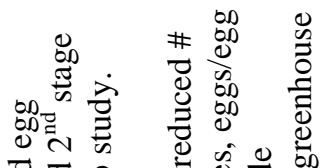

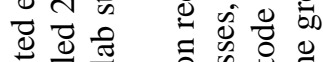

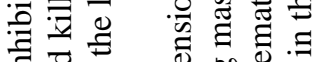

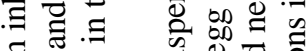

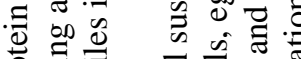

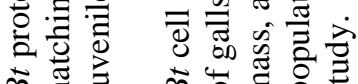

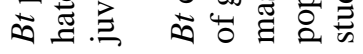

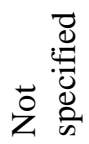

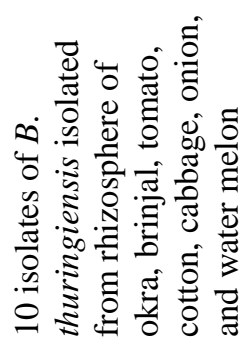

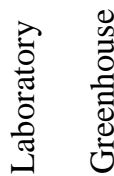

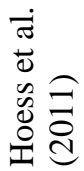

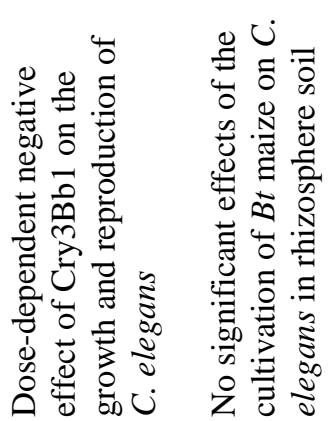

ᄅे

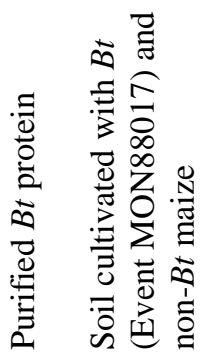

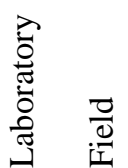
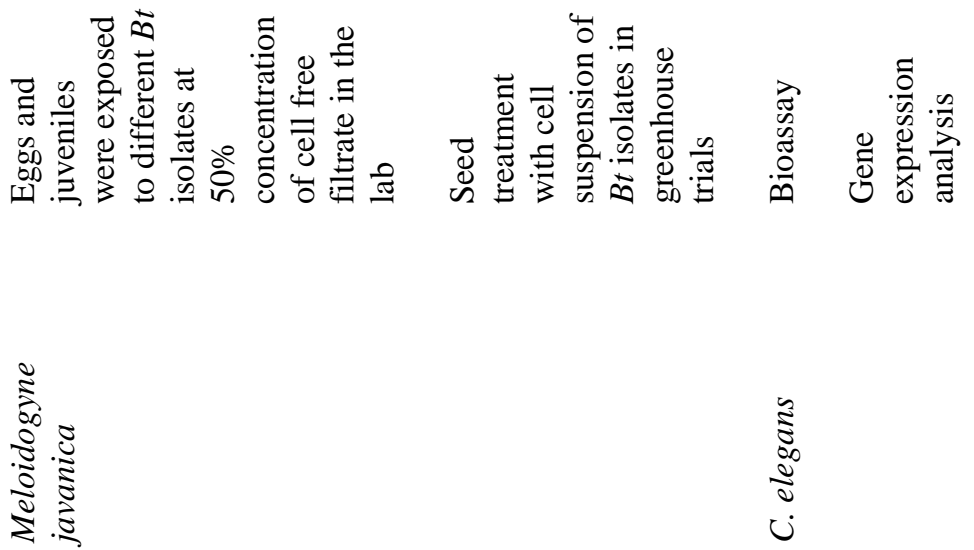


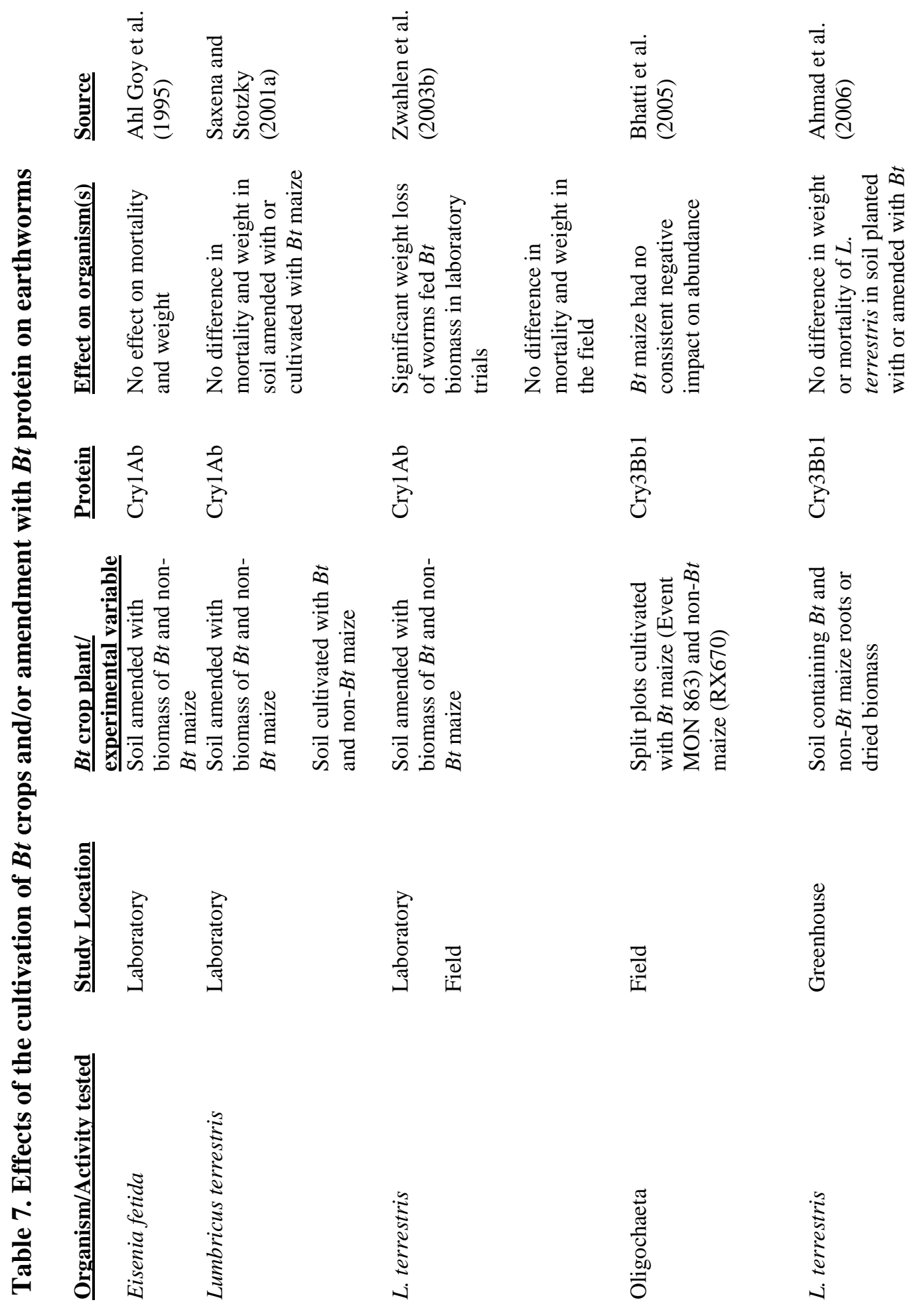




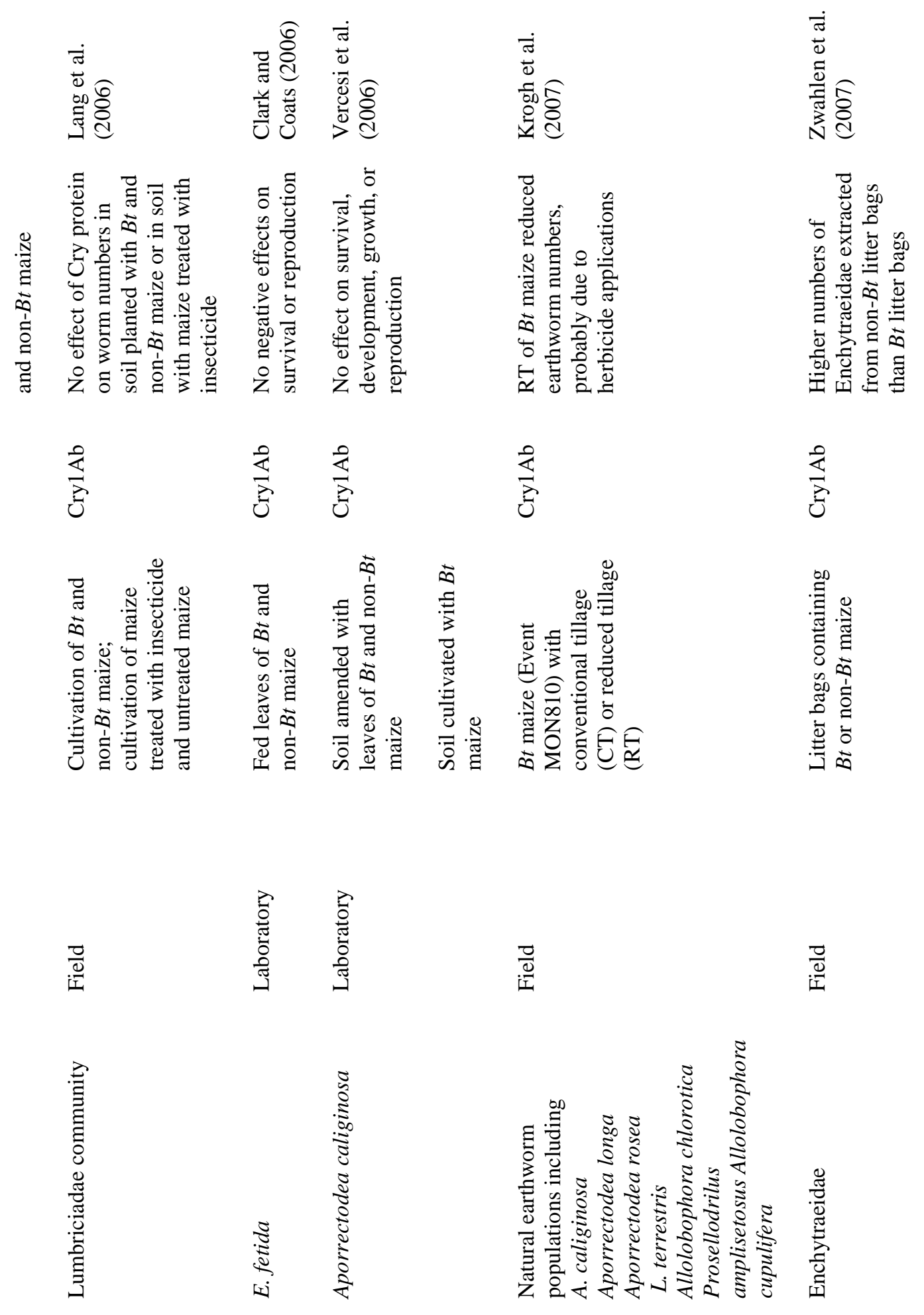




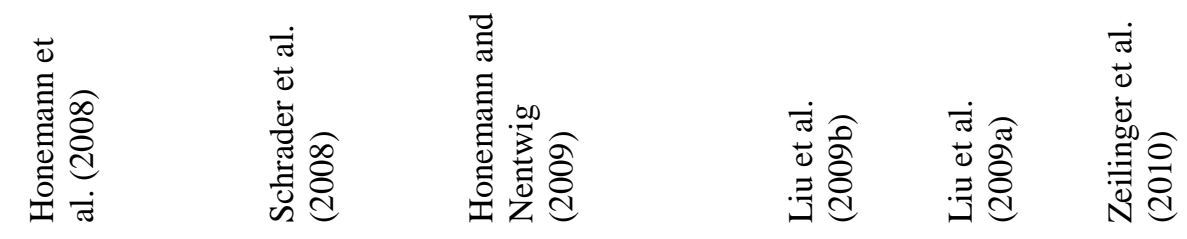

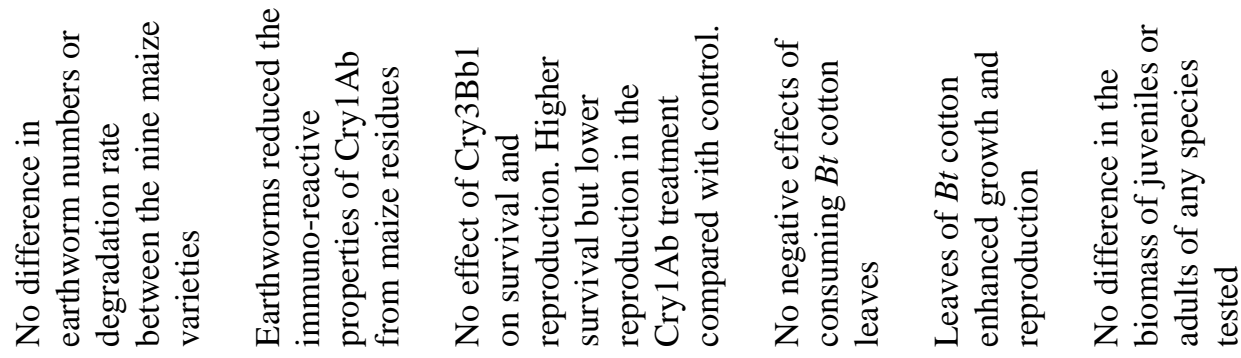

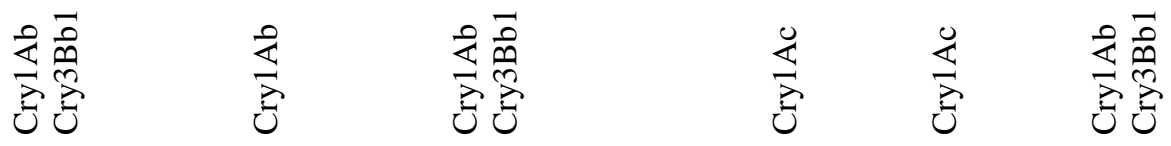

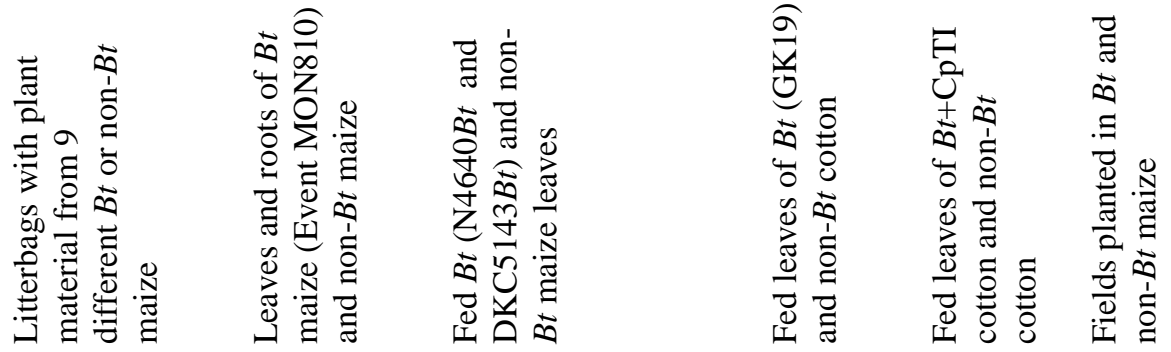
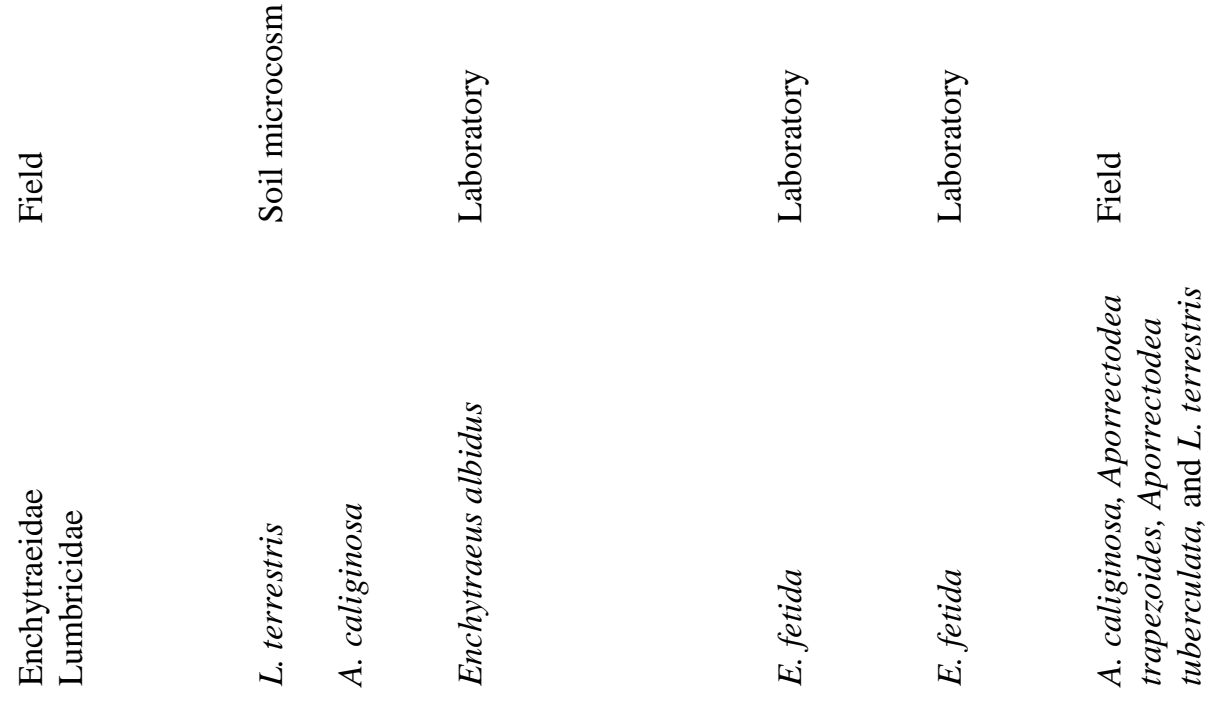


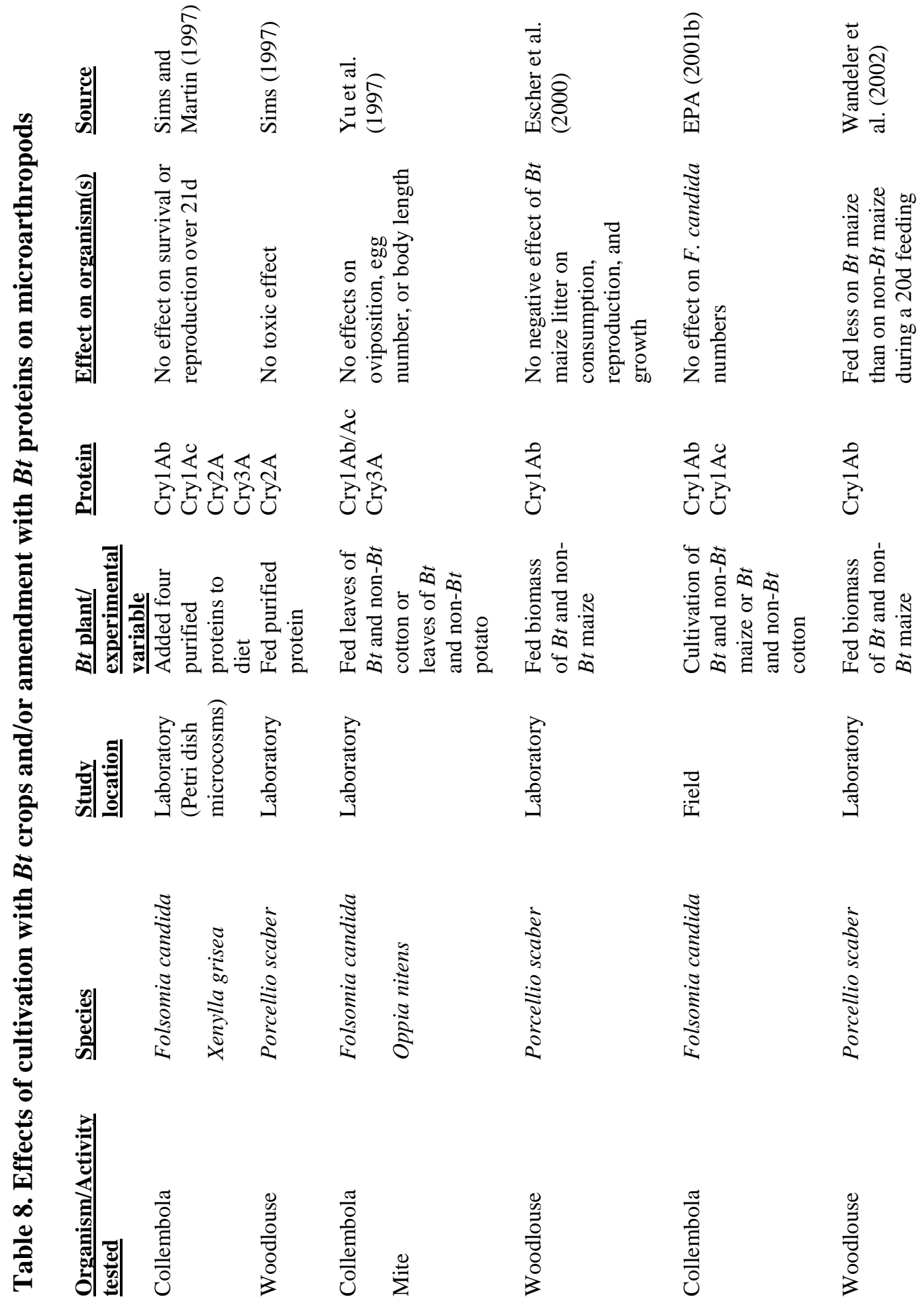




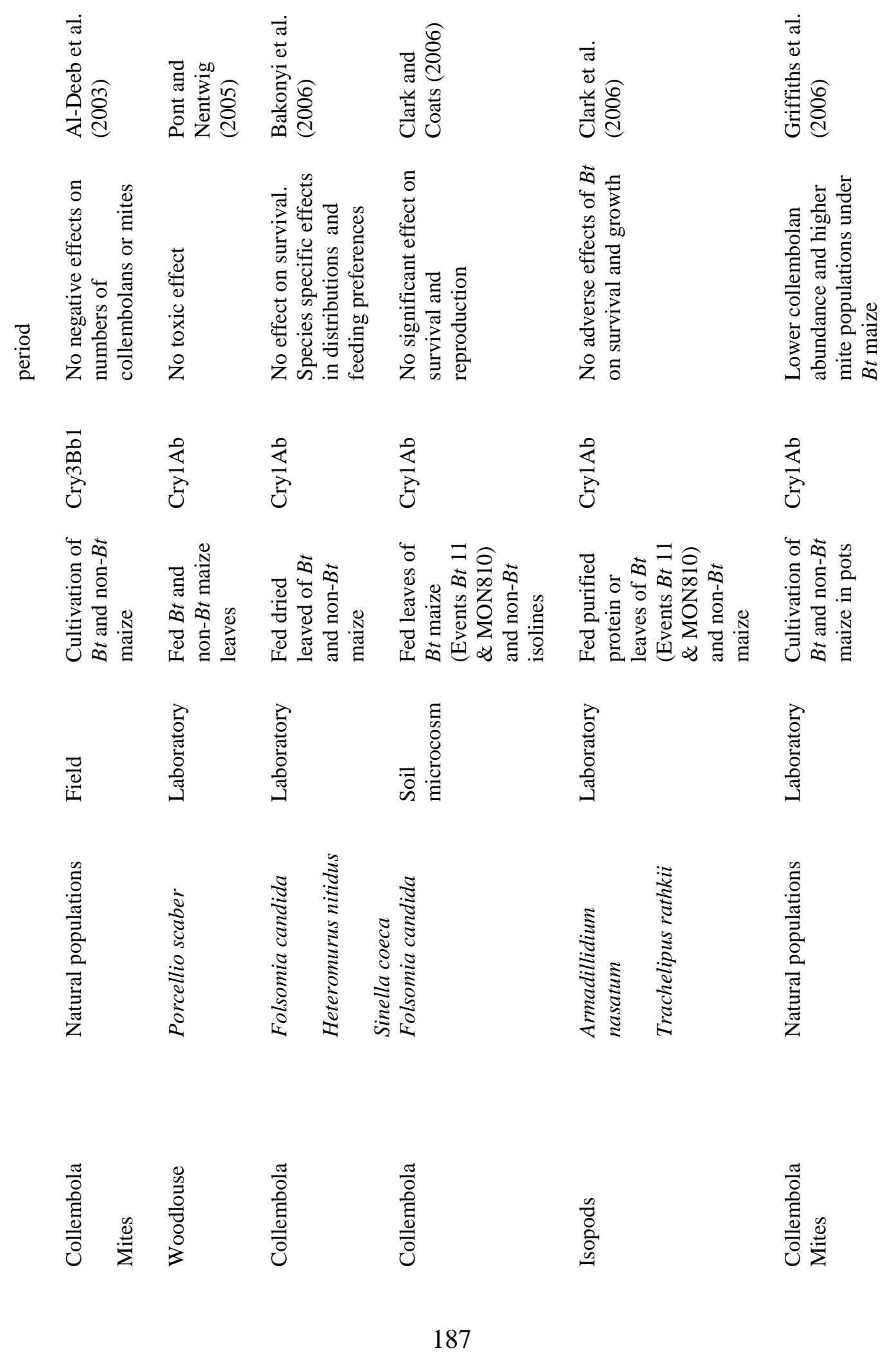



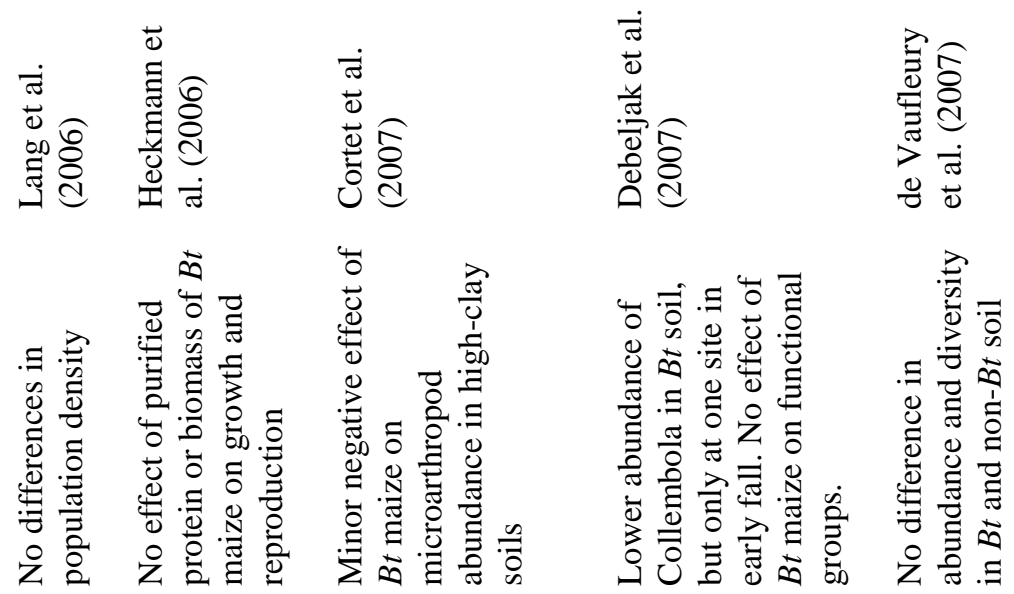

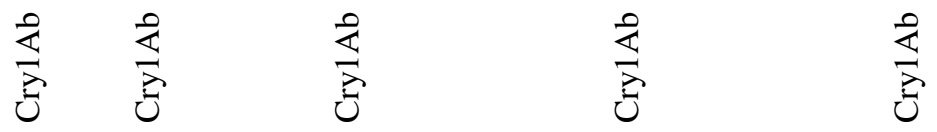

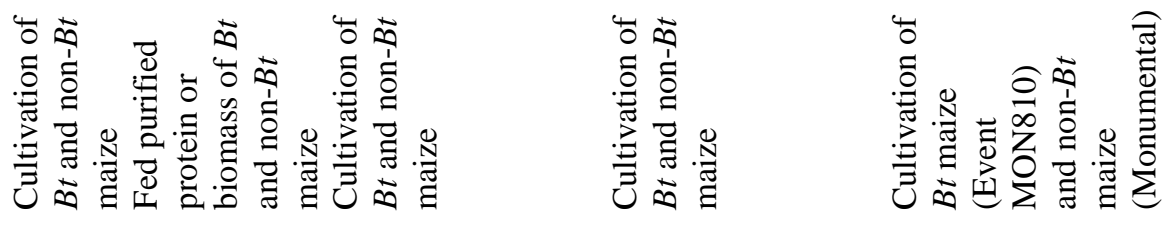

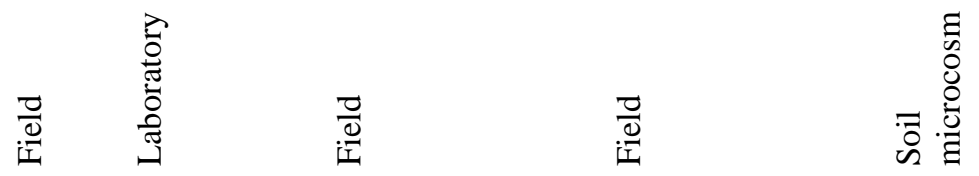

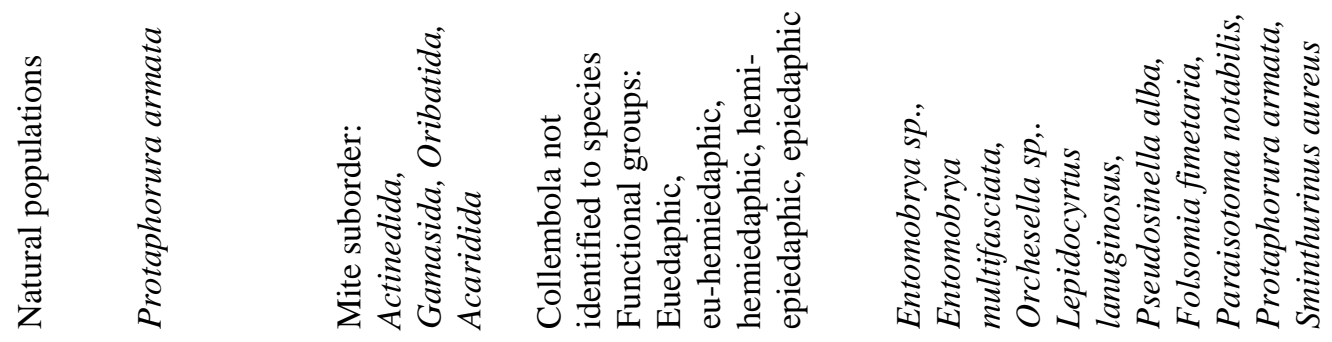

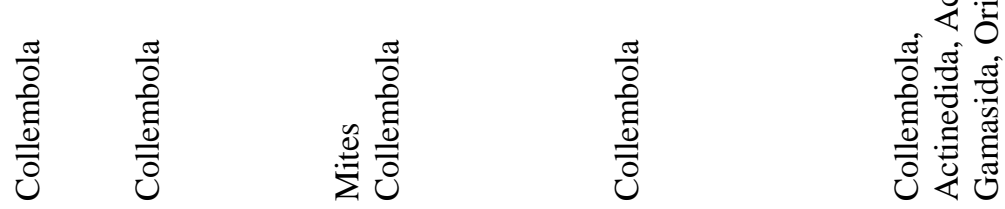

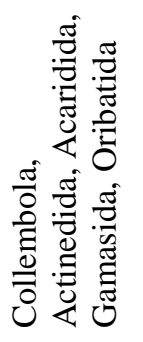



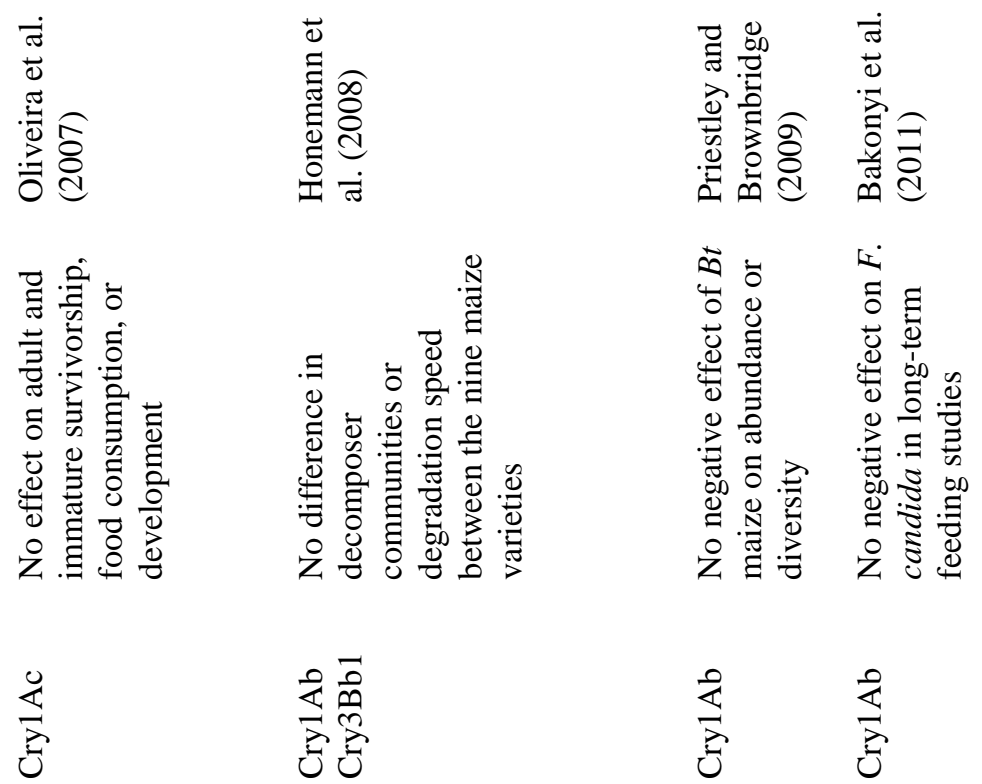

雍 是

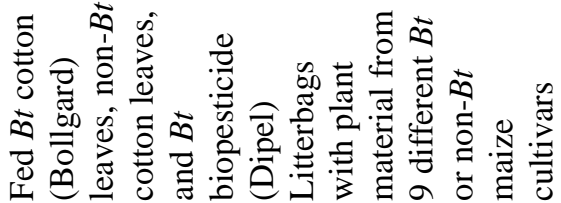
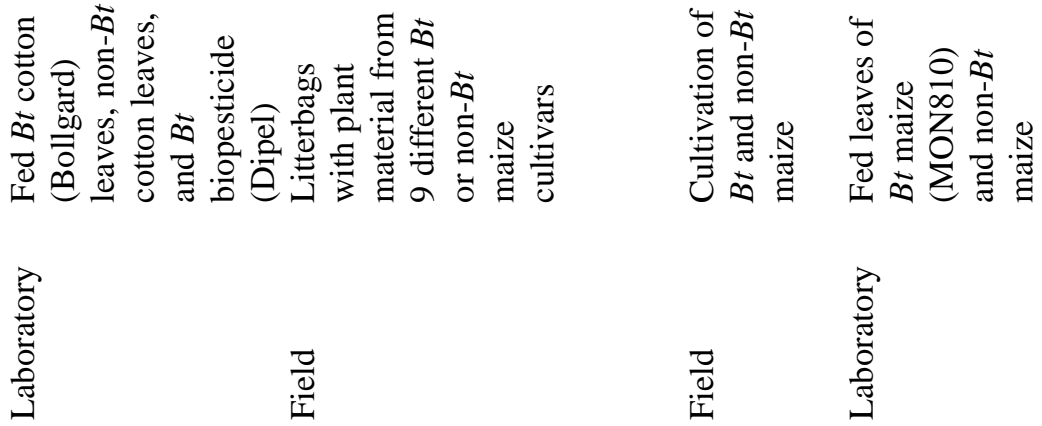

$\frac{0}{2}$

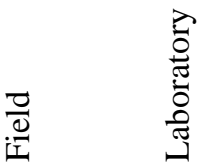
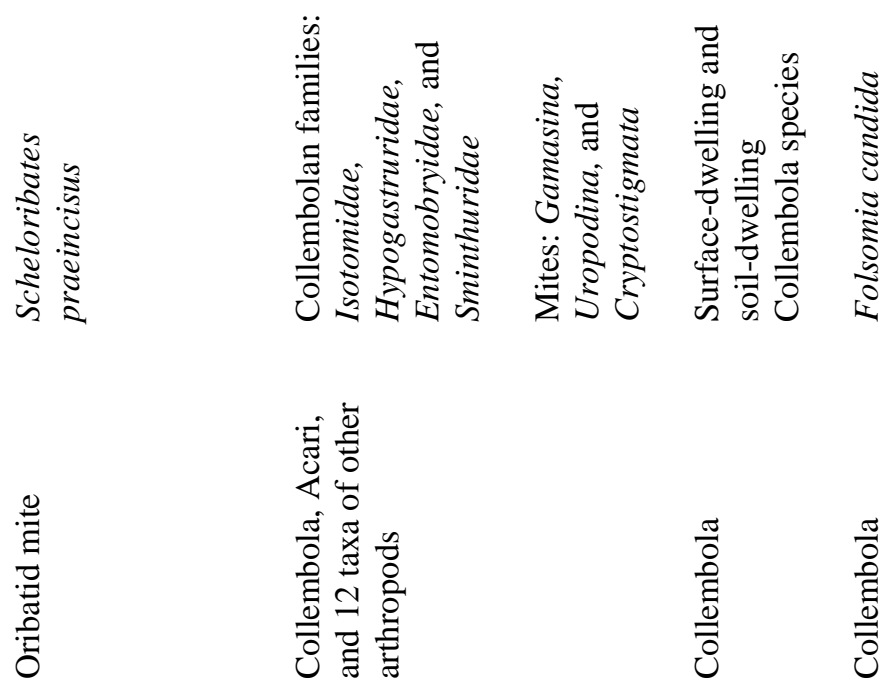

$\begin{array}{ll}\frac{\pi}{0} & \frac{\pi}{0} \\ \text { है } & \text { है } \\ 0 & 0 \\ 0 & 0\end{array}$ 


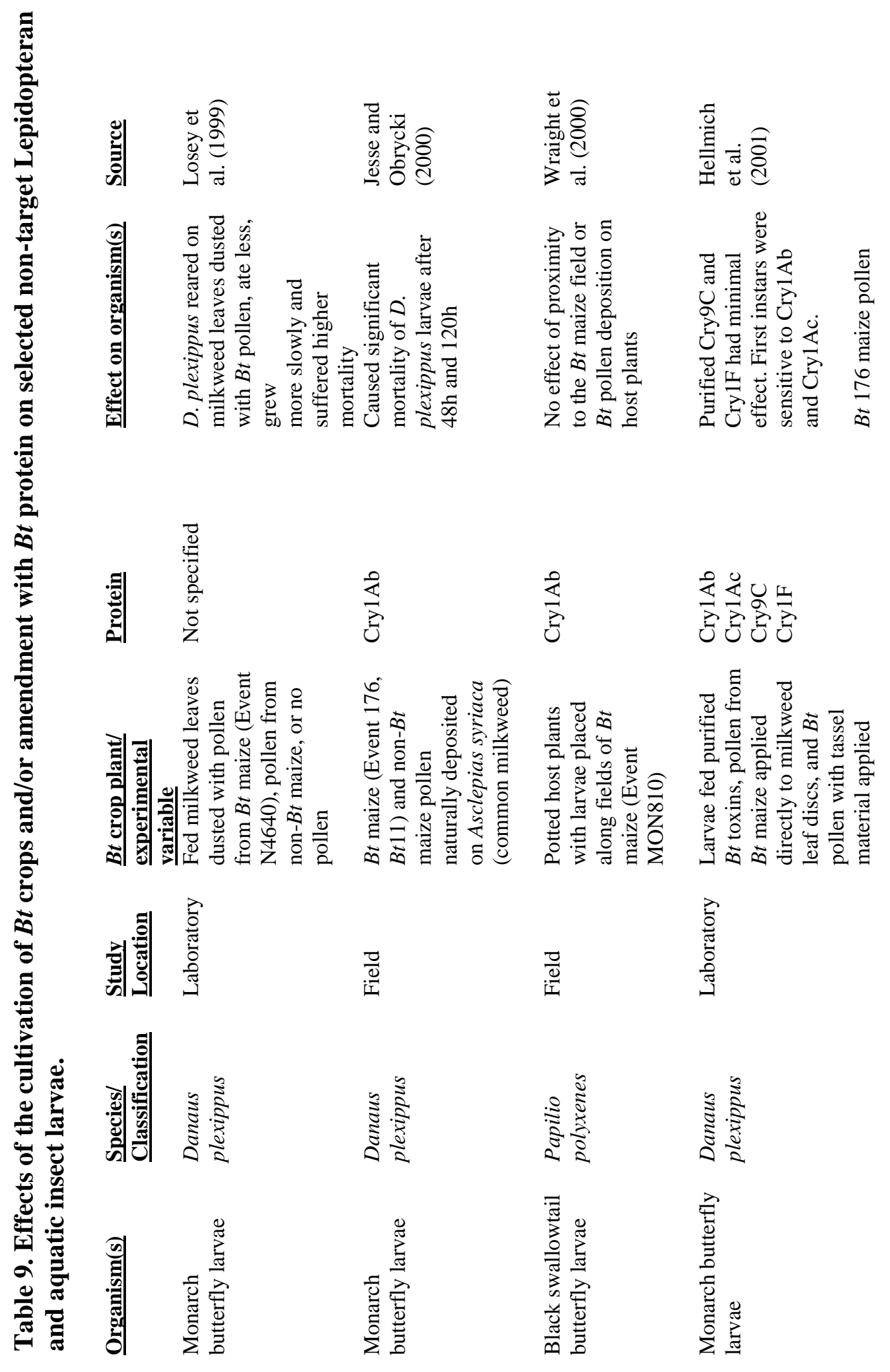



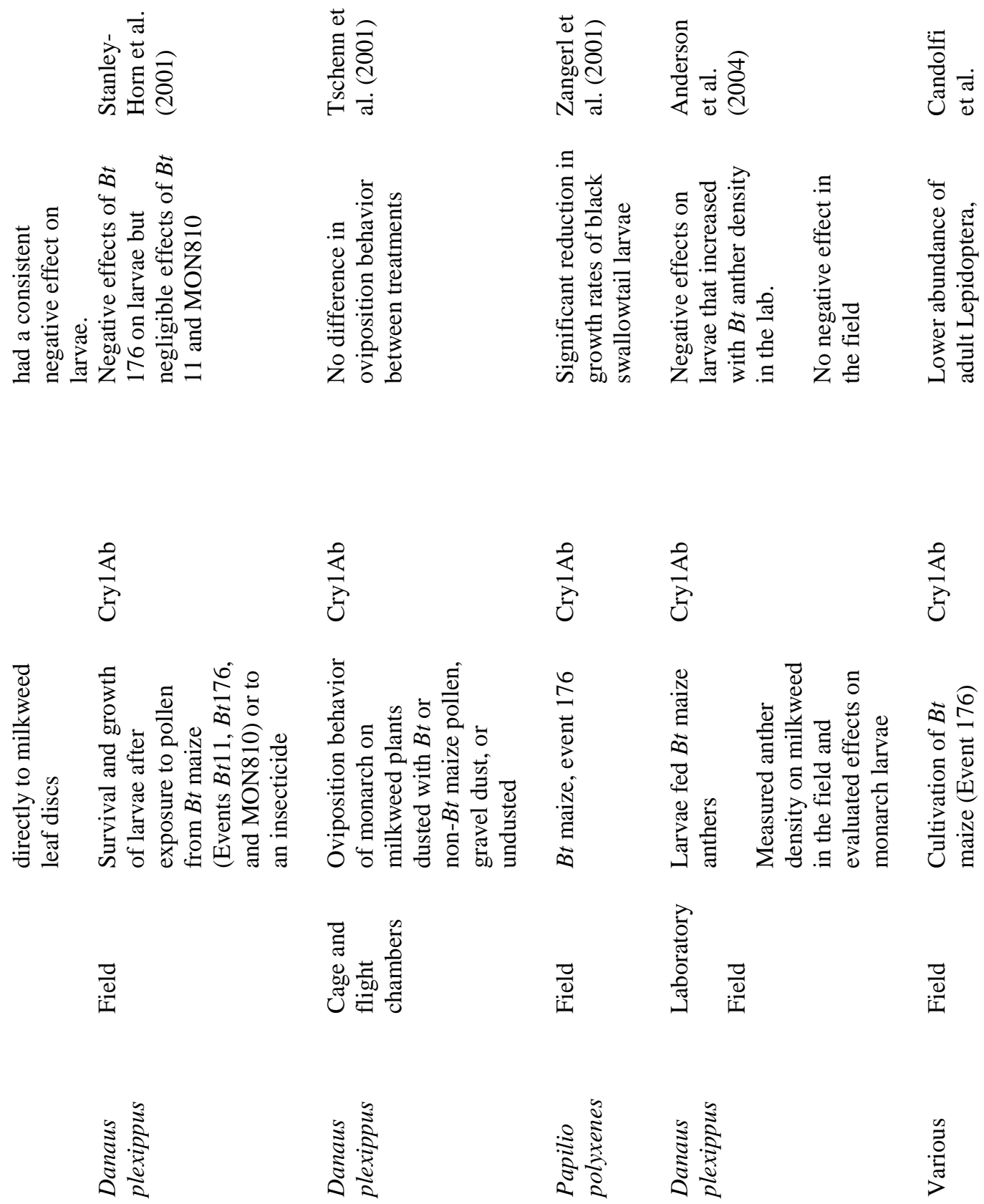

高
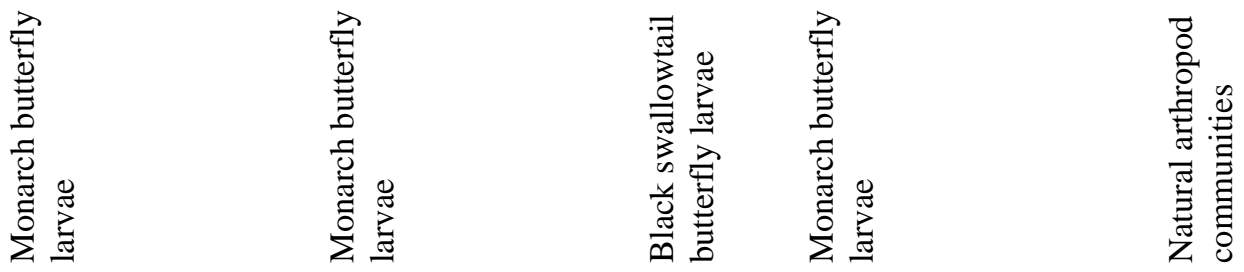

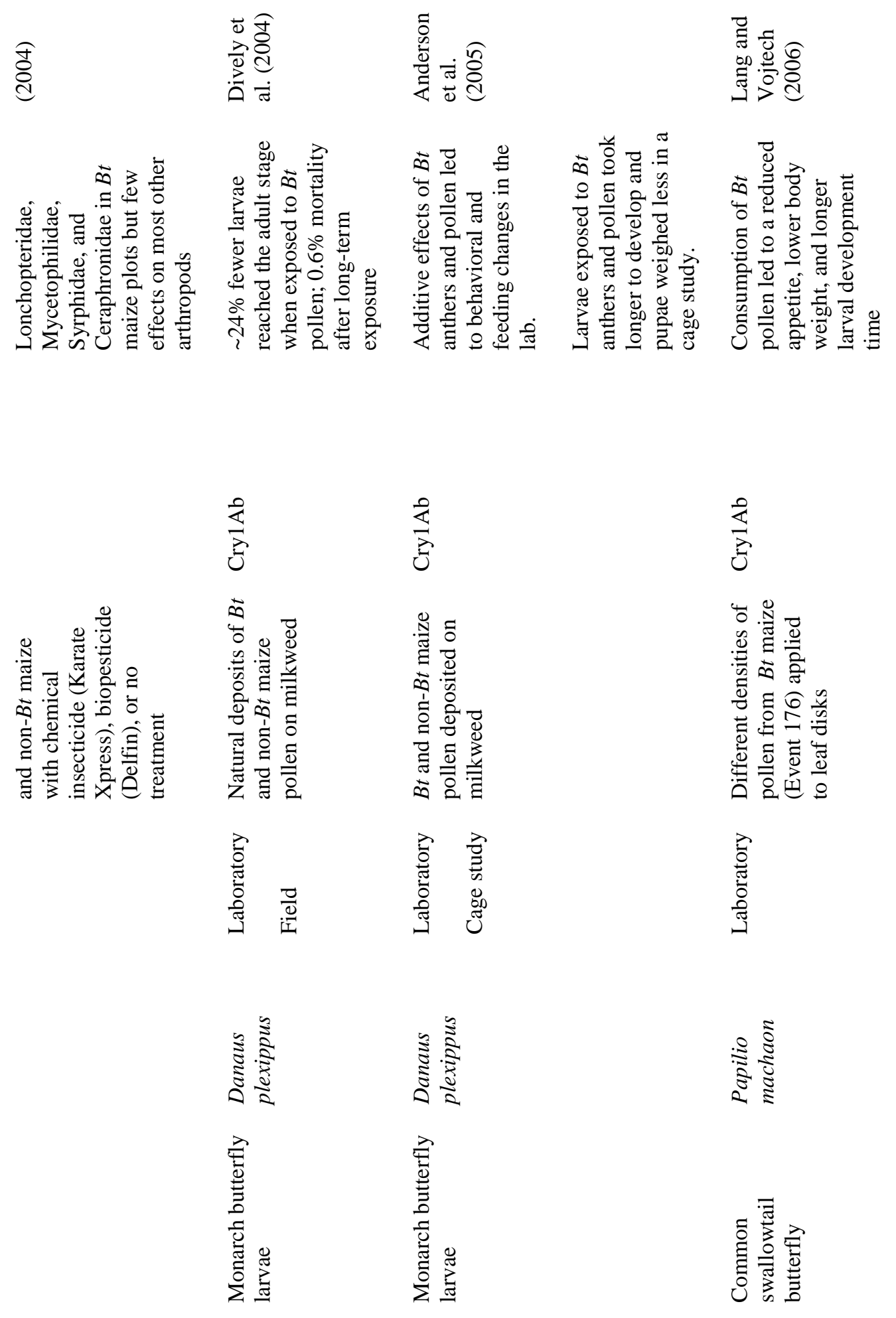


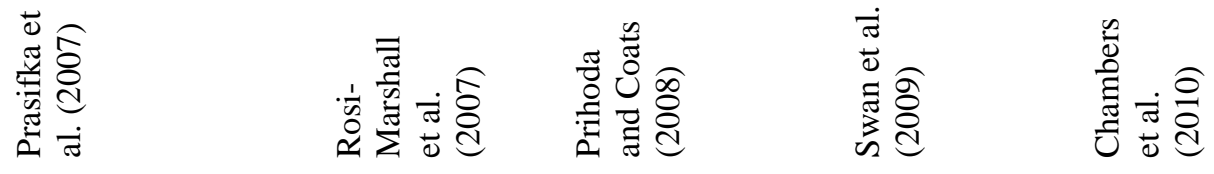

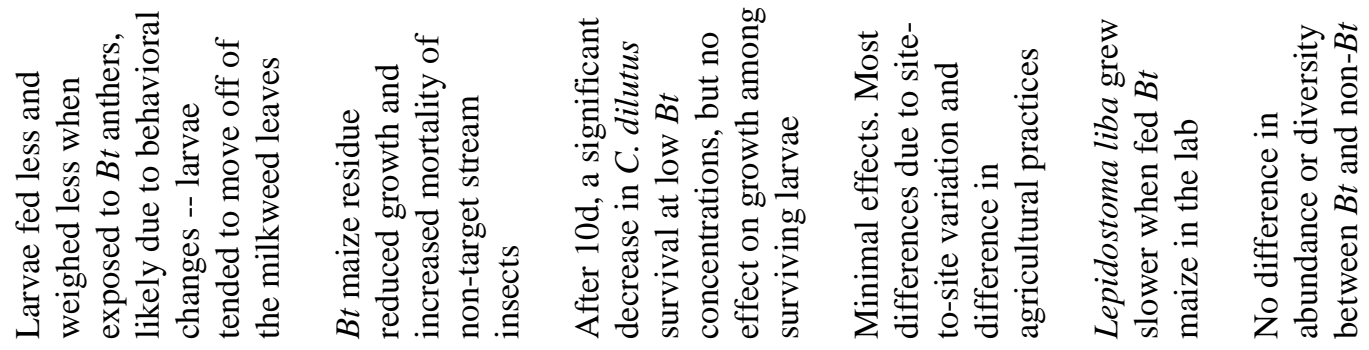
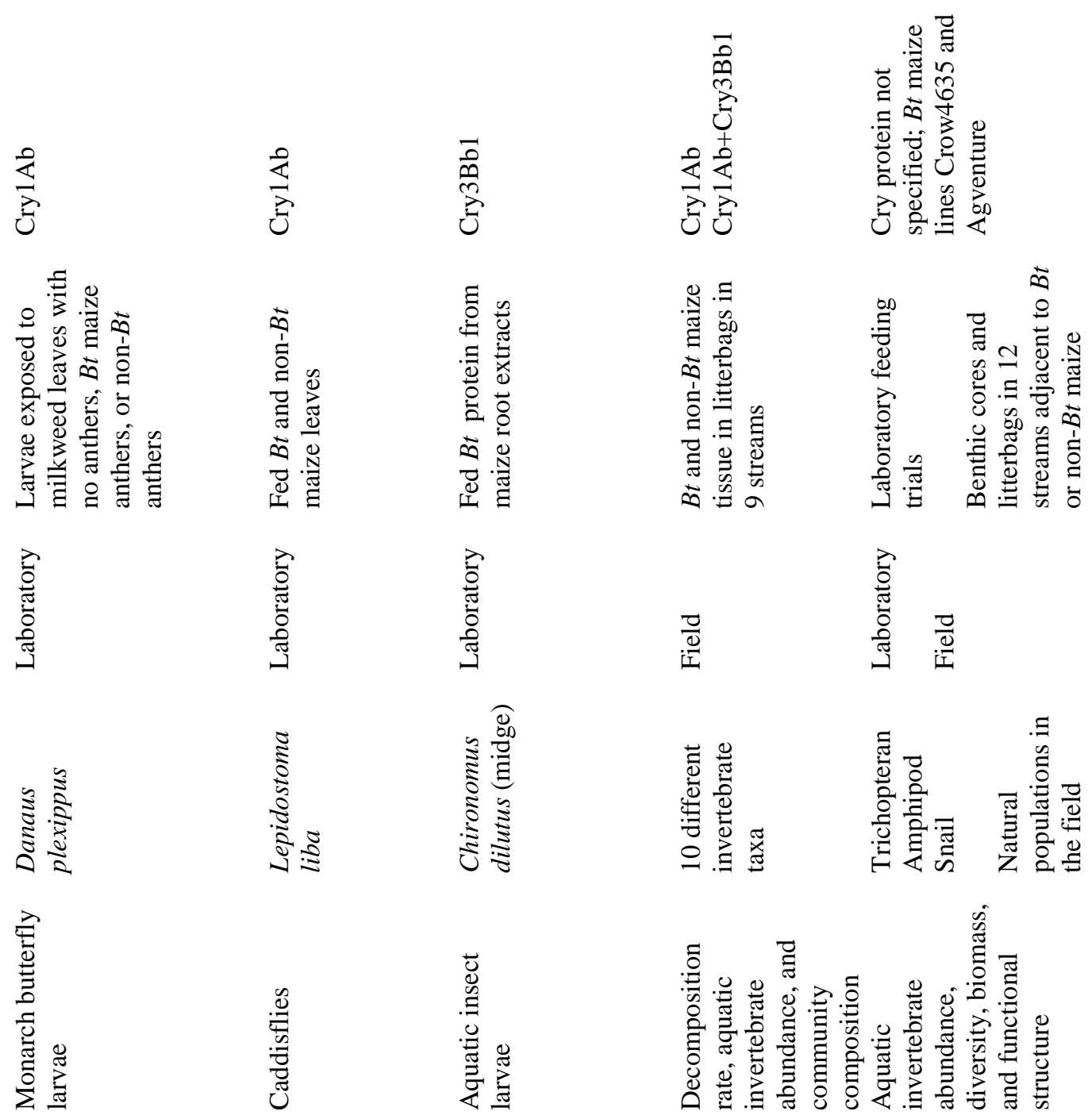


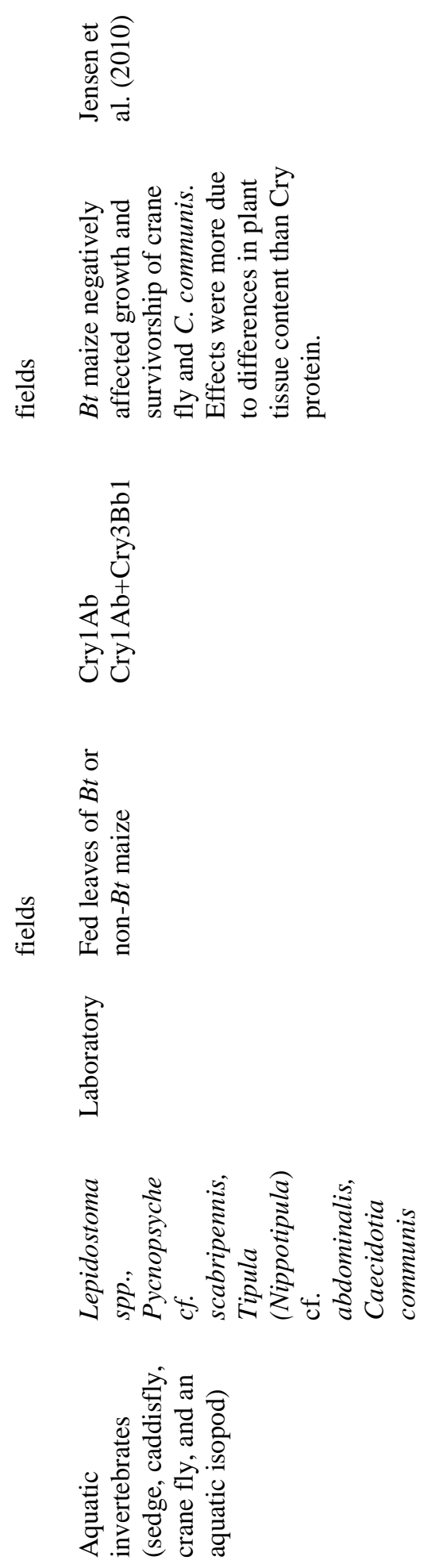




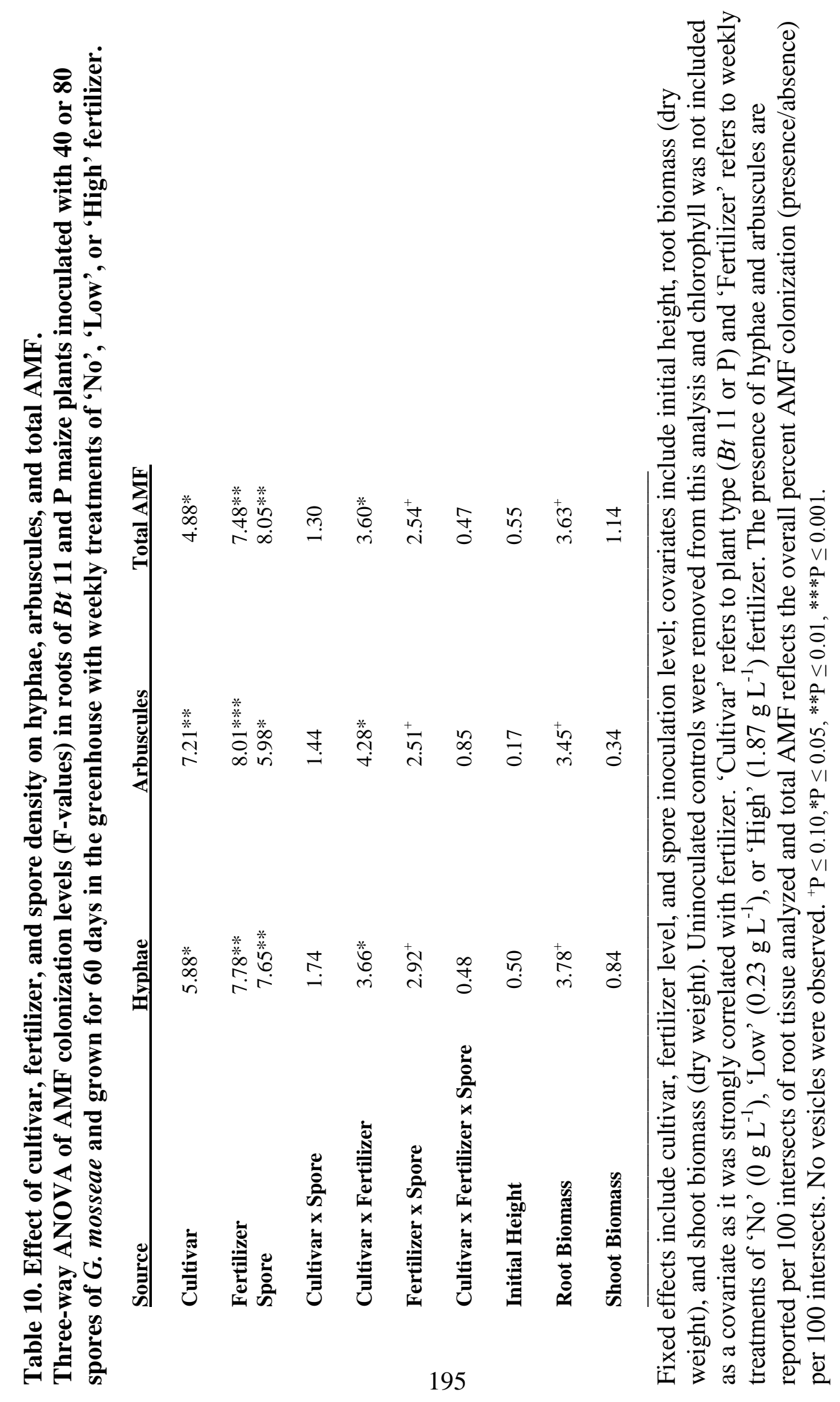




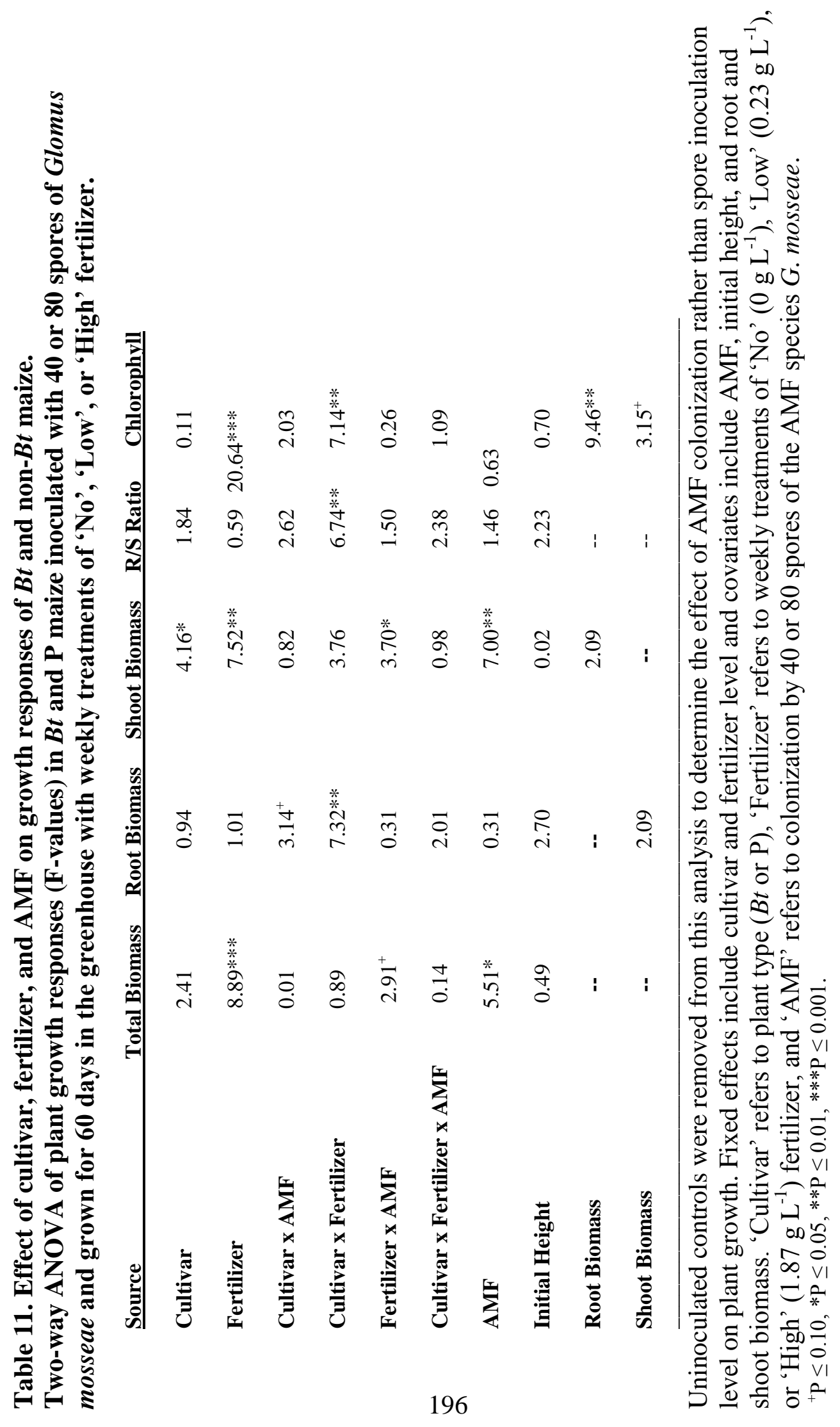




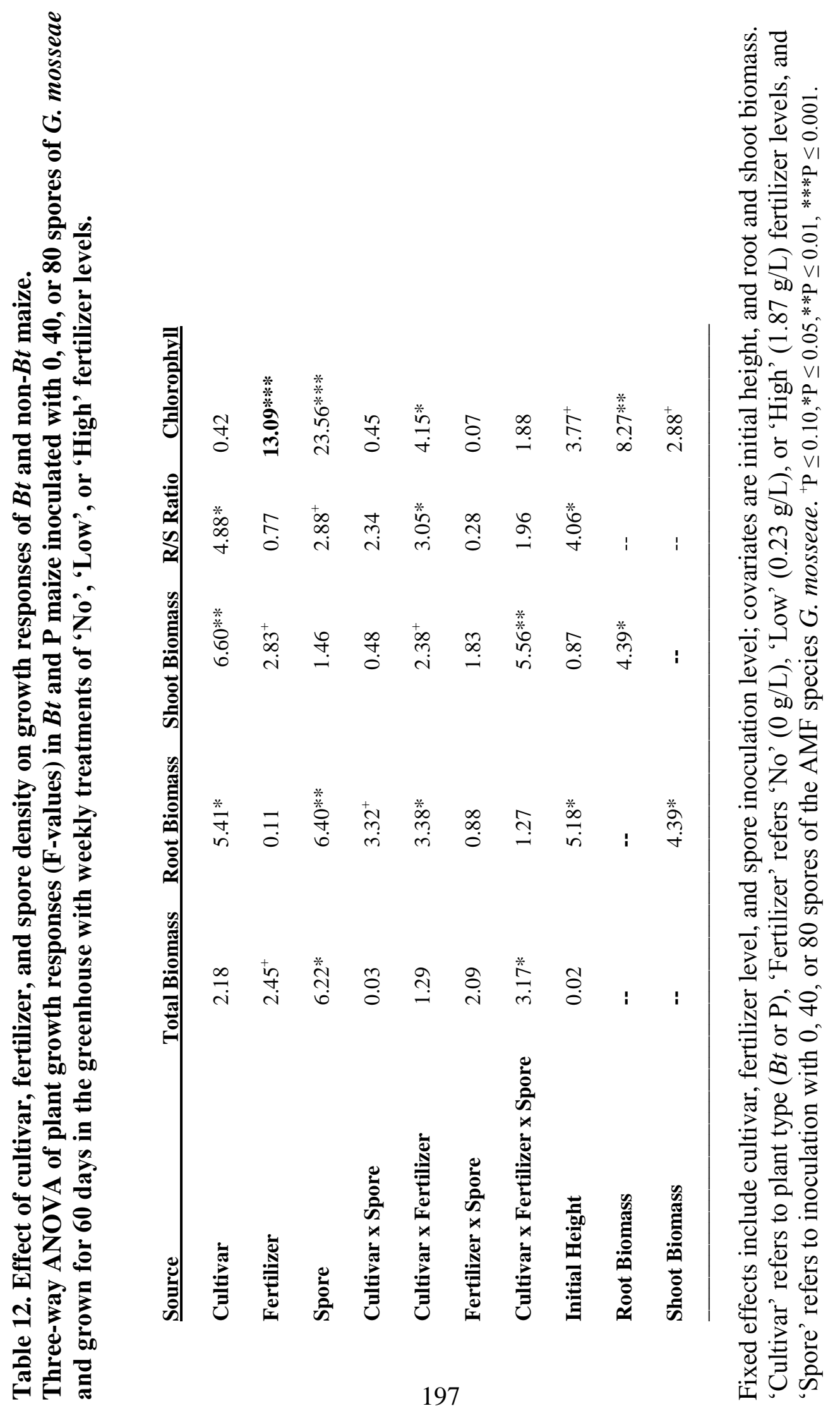


Table 13. $B t$ and non- $B t$ maize seeds used in greenhouse and field experiments. Fourteen different $B t$ and non-Bt maize lines were evaluated for AMF colonization in greenhouse and field experiments. $B t$ hybrids were assigned numbers B1-B9 and their corresponding non-Bt parental base-hybrids were assigned numbers P1-P5. Note that P2 was the parental line for $\mathrm{B} 2$ and $\mathrm{B5}, \mathrm{P3}$ was the parental line for the $\mathrm{B3}$ and B6, and P5 was the parental line for B7, B8, and B9.

\begin{tabular}{|c|c|c|c|c|c|}
\hline$\underline{B t}$ & $\frac{\text { Company; Plant }}{\underline{\text { ID }}}$ & Cry protein & Protection & $\frac{\text { Maize }}{\underline{\text { type }}}$ & $\frac{\text { Parental }}{\frac{\text { isoline }}{(\mathbf{P}) \#}}$ \\
\hline B1 & $\begin{array}{c}\text { Syngenta; } \\
\text { Attribute, } \\
\text { Bt 11: BC0805 }\end{array}$ & Cry1Ab & $\begin{array}{l}\text { European corn borer protection, } \\
\text { corn ear worm, fall armyworm; } \\
\text { Glufosinate herbicide tolerance }\end{array}$ & $\begin{array}{l}\text { Sweet } \\
\text { corn }\end{array}$ & $\mathrm{P} 1 *$ \\
\hline B2 & $\mathrm{N} / \mathrm{A}^{* *}$ & Cry34/35Ab1 & $\begin{array}{l}\text { Western corn rootworm, northern } \\
\text { corn rootworm, and Mexican corn } \\
\text { rootworm protection; Glufosinate } \\
\text { herbicide tolerance; Glyphosate } \\
\text { herbicide tolerance }\end{array}$ & Field corn & $\mathrm{P} 2$ \\
\hline B3 & N/A** & Cry34/35Ab1 & $\begin{array}{l}\text { Western corn rootworm, northern } \\
\text { corn rootworm, and Mexican corn } \\
\text { rootworm protection; Glufosinate } \\
\text { herbicide tolerance }\end{array}$ & Field corn & P3 \\
\hline B4 & N/A** & $\begin{array}{l}\text { Cry1F } \\
\text { Cry34/ } \\
35 \mathrm{Ab} 1\end{array}$ & $\begin{array}{l}\text { Western bean cutworm, corn } \\
\text { borer, black cutworm and fall } \\
\text { army worm resistance; } \\
\text { Glufosinate herbicide tolerance. } \\
\text { Western corn rootworm, Northern } \\
\text { corn rootworm protection; } \\
\text { Glyphosate herbicide tolerance }\end{array}$ & Field corn & $\mathrm{P} 4$ \\
\hline B5 & N/A** & Cry1F & $\begin{array}{l}\text { Western bean cutworm, corn } \\
\text { borer, black cutworm and fall } \\
\text { armyworm resistance; Glyphosate } \\
\text { herbicide tolerance; Glufosinate } \\
\text { herbicide tolerance }\end{array}$ & Field corn & $\mathrm{P} 2$ \\
\hline B6 & N/A** & Cry1F & $\begin{array}{l}\text { Western bean cutworm, corn } \\
\text { borer, black cutworm and fall } \\
\text { armyworm resistance; Glyphosate } \\
\text { herbicide tolerance; Glufosinate } \\
\text { herbicide tolerance }\end{array}$ & Field corn & P3 \\
\hline B7 & $\begin{array}{c}\text { Monsanto; } \\
\text { DKC51-41 Mon } \\
\text { 863, Nk603*** }\end{array}$ & Cry3Bb1 & $\begin{array}{c}\text { Corn rootworm protection; } \\
\text { Glyphosate herbicide tolerance } \\
\text { (RR2) }\end{array}$ & Field corn & $\begin{array}{c}\text { P5 } \\
\text { DKC51- } \\
45(\text { RR2) }\end{array}$ \\
\hline B8 & $\begin{array}{c}\text { Monsanto; } \\
\text { DKC50-20 Mon } \\
810, \text { Nk603*** }\end{array}$ & Cry1Ab & $\begin{array}{l}\text { European corn borer protection; } \\
\text { Glyphosate herbicide tolerance } \\
\text { (RR2) }\end{array}$ & Field corn & $\begin{array}{c}\text { P5 } \\
\text { DKC51- } \\
45(\text { RR2) }\end{array}$ \\
\hline B9 & $\begin{array}{c}\text { Monsanto; } \\
\text { DKC51-39 Mon } \\
863,\end{array}$ & $\begin{array}{l}\text { Cry1Ab } \\
\text { Cry3Bb1 }\end{array}$ & $\begin{array}{c}\text { Corn rootworm, European corn } \\
\text { borer protection; Glyphosate } \\
\text { herbicide tolerance (RR2) }\end{array}$ & Field corn & $\begin{array}{c}\text { P5 } \\
\text { DKC51- } \\
45(\mathrm{RR} 2) \\
\end{array}$ \\
\hline
\end{tabular}




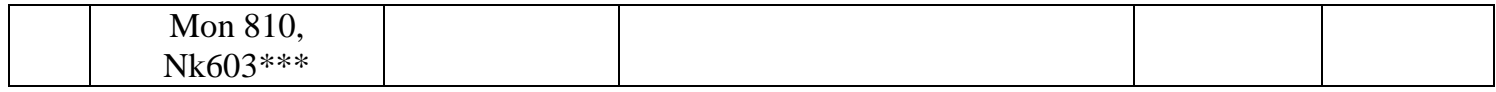

* The $B t 11$ transgene was backcrossed into one of the parents of Providence (P1) to create the variety BC0805. This $B t 11$ cultivar was transformed using the plasmid pZ01502 (containing Cry1Ab, pat, and amp genes) to express the Cry1 Ab protein of Bacillus thuringiensis.

** Our seed agreement prohibits us from disclosing information about this seed industry representative, the genetics of the $B t$ and parental isolines, or other information related to the seeds provided for this study.

*** Nk603 is the gene for Round Up Ready 2 (RR2) Glyphosate herbicide tolerance. 


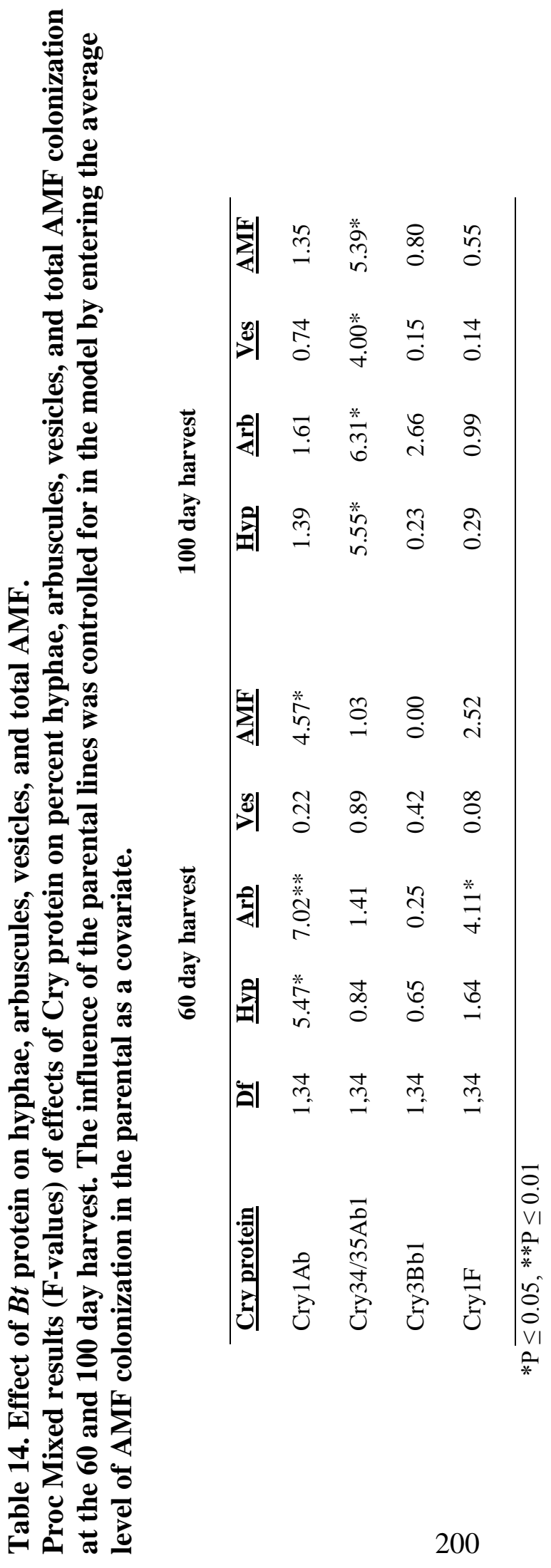


Table 15. Effect of plant type (Bt or P) on AMF 60, 100, and $130 \mathrm{~d}$ after sowing. Proc Mixed results (F-values) of the effects of plant type (Bt or non-Bt maize) on colonization of roots by AMF hyphae, arbuscules, and/or vesicles, and total percentage AMF colonization (presence/absence of any fungal structure per 100 intersects) at the $60 \mathrm{~d}, 90 \mathrm{~d}$, and $130 \mathrm{~d}$ harvests.

\begin{tabular}{|c|c|c|c|}
\hline AMF Response & df & F-value & P-value \\
\hline 60 d harvest & & & \\
\hline Hyphae & 1,4 & 0.14 & 0.73 \\
\hline Arbuscules & 1,4 & 0.13 & 0.73 \\
\hline Vesicles & 1,4 & 1.14 & 0.34 \\
\hline Total AMF & 1,4 & 0.20 & 0.68 \\
\hline 90 d harvest & & & \\
\hline Hyphae & 1,4 & 2.03 & 0.23 \\
\hline Arbuscules & 1,4 & 2.34 & 0.20 \\
\hline Vesicles & 1,4 & 0.23 & 0.66 \\
\hline Total AMF & 1,4 & 2.11 & 0.22 \\
\hline 130 d harvest & & & \\
\hline Hyphae & 1,4 & 0.15 & 0.72 \\
\hline Arbuscules & 1,4 & 0.06 & 0.81 \\
\hline Vesicles & 1,4 & 0.49 & 0.52 \\
\hline Total AMF & 1,4 & 0.15 & 0.72 \\
\hline
\end{tabular}


Table 16. Effect of AMF on growth response of $B t$ and $P$ maize at 60,90 , and $130 \mathrm{~d}$. Proc Mixed results (F-values) of the effects of percentage AMF colonization in roots (presence of AMF hyphae, arbuscules, and/or vesicles per 100 intersects) on maize growth (root dry weight, shoot dry weight, leaf chlorophyll content, ear number, and ear dry weight) at the $60 \mathrm{~d}, 90 \mathrm{~d}$, and $130 \mathrm{~d}$ harvests.

\begin{tabular}{|c|c|c|c|}
\hline Growth Response & df & F-value & P-value \\
\hline 60 d harvest & & & \\
\hline Root biomass & 1,51 & 0.20 & 0.66 \\
\hline Shoot biomass & 1,51 & 0.21 & 0.65 \\
\hline Leaf Chl content & 1,52 & 1.05 & 0.31 \\
\hline 90 d harvest & & & \\
\hline Root biomass & 1,167 & 0.03 & 0.87 \\
\hline Shoot biomass & 1,168 & 0.59 & 0.44 \\
\hline Leaf Chl content & 1,169 & 1.81 & 0.18 \\
\hline Ear number & 1,168 & 0.17 & 0.68 \\
\hline 130 d harvest & & & 0.31 \\
\hline Root biomass & 1,89 & 0.01 & 0.94 \\
\hline Shoot biomass & 1,89 & $0.03 *$ \\
\hline Leaf Chl content & 1,90 & 4.61 & 0.74 \\
\hline Ear number & 1,89 & 0.11 & 0.22 \\
\hline Ear weight & 1,88 & 1.50 & \\
\hline
\end{tabular}

$* \mathrm{P} \leq 0.05$. 
Table 17. Effect of plant type of growth response 60, 90, and $130 \mathrm{~d}$ after sowing. Proc Mixed results (F-values) of the effects of plant type (Bt or non-Bt maize) on plant growth (root dry weight, shoot dry weight, leaf chlorophyll content, ear number, and ear dry weight) at the $60 \mathrm{~d}, 90 \mathrm{~d}$, and $130 \mathrm{~d}$ harvests.

\begin{tabular}{|c|c|c|c|}
\hline Growth Response & df & $\underline{\text { F-value }}$ & P-value \\
\hline 60 d harvest & & & \\
\hline Root biomass & 1,4 & 0.22 & 0.66 \\
\hline Shoot biomass & 1,4 & 0.00 & 0.96 \\
\hline Leaf Chl content & 1,4 & 0.66 & 0.46 \\
\hline 90d harvest & & & \\
\hline Root biomass & 1,4 & 0.88 & 0.40 \\
\hline Shoot biomass & 1,4 & 0.58 & 0.49 \\
\hline Leaf Chl content & 1,4 & 1.82 & 0.25 \\
\hline Ear number & 1,4 & 1.44 & 0.30 \\
\hline 130 d harvest & & & \\
\hline Root biomass & 1,4 & 0.01 & 0.92 \\
\hline Shoot biomass & 1,4 & 0.08 & 0.79 \\
\hline Leaf Chl content & 1,4 & 1.22 & 0.33 \\
\hline Ear number & 1,4 & 1.03 & 0.34 \\
\hline Ear weight & 1,4 & 0.46 & 0.53 \\
\hline
\end{tabular}


Table 18. Tissue-specific stains used to detect lignin and suberin in cross-sections of Bt 11 (Attribute) maize (sweetcorn) and its parental isoline (Providence).

\begin{tabular}{|l|l|l|l|}
\hline Stain & $\underline{\text { Purpose }}$ & $\underline{\text { Light }}$ & Citation \\
\hline Toluidine Blue & $\begin{array}{l}\text { Used to detect potential } \\
\text { differences in cell wall } \\
\text { layers between } B t \text { and P } \\
\text { maize }\end{array}$ & $\begin{array}{l}\text { White and } \\
\text { fluorescent }\end{array}$ & $\begin{array}{l}\text { (Saxena and Stotzky } \\
\text { 2001) }\end{array}$ \\
\hline Safranin O & $\begin{array}{l}\text { Used to detect potential } \\
\text { differences in lignin } \\
\text { content of } B t \text { and P roots }\end{array}$ & Fluorescent & $\begin{array}{l}\text { (De Micco and Aronne } \\
\text { 2007) }\end{array}$ \\
\hline $\begin{array}{l}\text { Berberine Hemi-Sulfate } \\
\text { and Analine Blue }\end{array}$ & $\begin{array}{l}\text { Used to detect } \\
\text { differences in suberin } \\
\text { and lignin between } B t \\
\text { and P root samples }\end{array}$ & Fluorescent & (Brundrett et al. 1988) \\
\hline $\begin{array}{l}\text { Sudan Red III } \\
\text { Bsed to view suberin in }\end{array}$ & $\begin{array}{l}\text { White and } \\
\text { fluorescent }\end{array}$ & (Zeier et al. 1999) \\
\hline $\begin{array}{l}\text { Trypan blue + } \\
\text { Berberine- } \\
\text { Hemisulfate/Analine } \\
\text { blue, Toluidine Blue, or } \\
\text { Sudan Red III }\end{array}$ & $\begin{array}{l}\text { To determine at what } \\
\text { layer (epidermis, } \\
\text { exodermis) appressoria } \\
\text { is formed and/or where } \\
\text { the infection peg aborted }\end{array}$ & $\begin{array}{l}\text { White and } \\
\text { fluorescent }\end{array}$ & $\begin{array}{l}\text { Developed in Cruzan } \\
\text { lab to visualize both } \\
\text { mycorrhizal structures } \\
\text { and suberized or } \\
\text { lignified plant tissue }\end{array}$ \\
\hline
\end{tabular}




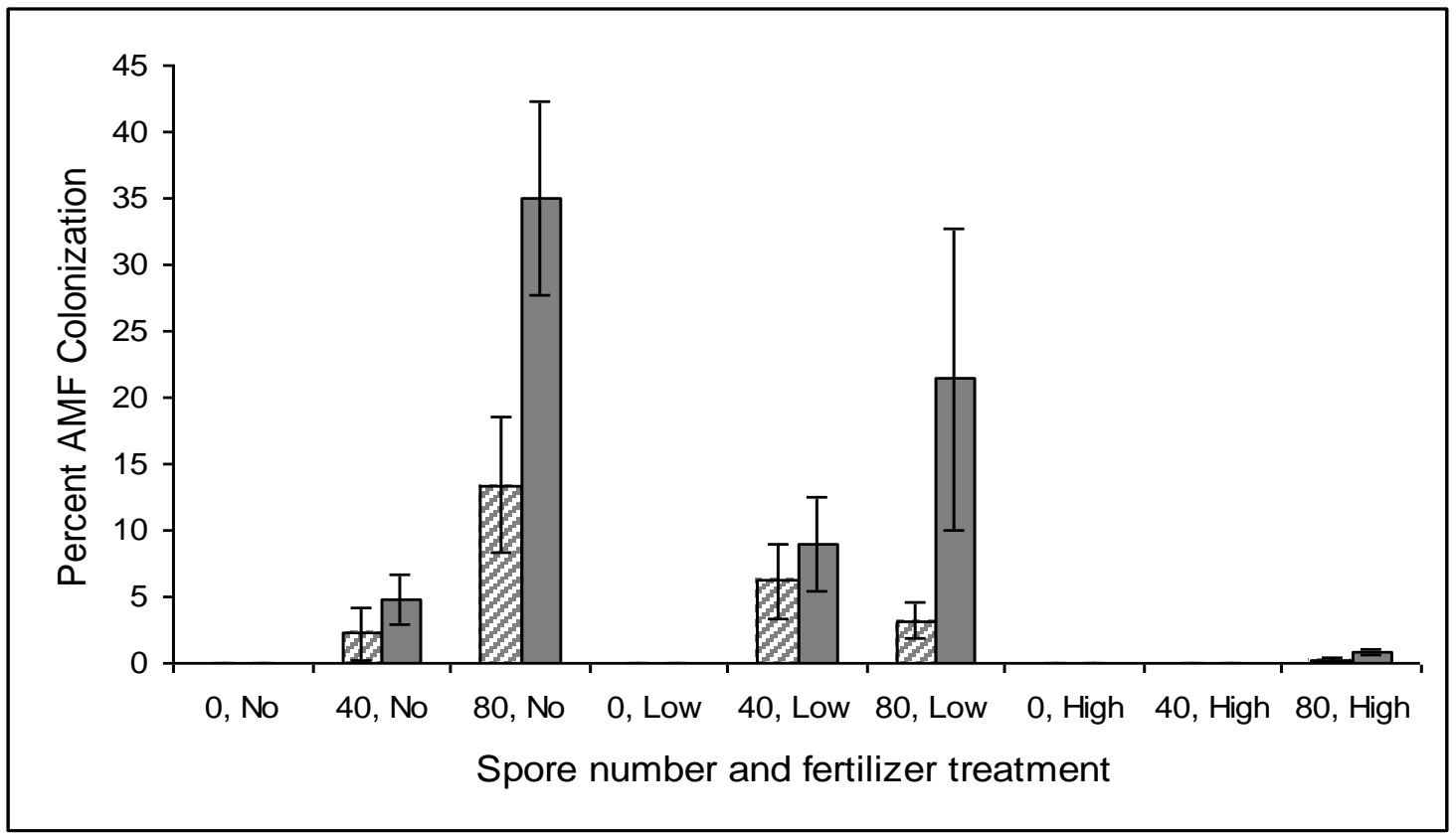

Figure 1. Effect of plant type, spore density, and fertilizer on AMF colonization.

Percent colonization by the AMF species Glomus mosseae in Bt maize (Event Bt 11) and non-Bt maize (parental isoline, Providence) inoculated with 0,40 , or 80 spores of Glomus mosseae and grown for 60 days in a greenhouse with weekly treatments of 'No' $(0 \mathrm{~g} / \mathrm{L})$, 'Low' $(0.23 \mathrm{~g} / \mathrm{L})$, or 'High' $(1.87 \mathrm{~g} / \mathrm{L})$ of a complete fertilizer. Striped bars represent the means (+/- SEM) of $B t 11$ plants and solid bars represent the means $(+/$ - SEM) of non- $B t$ maize plants. $* \mathrm{P} \leq 0.05, \mathrm{n}=5$ for each bar. 
a) No fertilizer

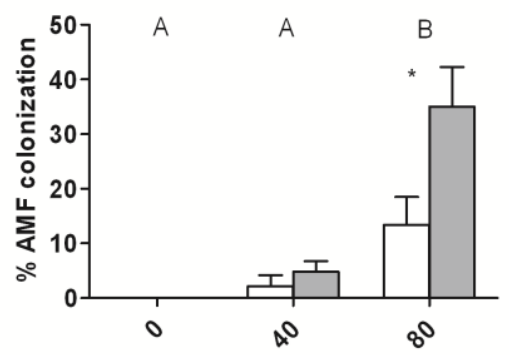

b) Low fertilizer

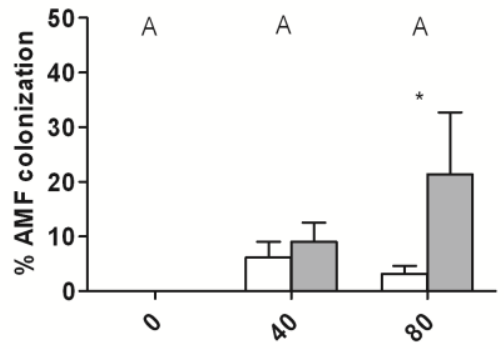

c) High fertilizer

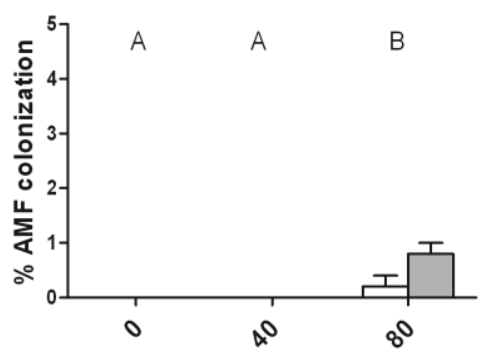

Figure 2. Effect of fertilizer and spore density on AMF colonization in $B t$ and non-Bt maize. Percent AMF colonization in $B t 11$ and $P$ maize roots inoculated with 0 , 40, or 80 spores of Glomus mosseae and grown for 60 days with weekly treatments of (a) 'No', (b) 'Low', or (c) 'High' fertilizer levels. Open bars represent the means $( \pm \mathrm{SE})$ of transgenic $B t 11$ plants and solid bars represent the means $( \pm \mathrm{SE})$ of $P$ parental plants. $* P<\mathbf{0 . 0 5}, \mathrm{n}=5$ for each bar. Note the change in $y$-axis scale for Fig. 2.1c. Uppercase letters reflect the results of the Tukey multiple range test; means with a different letter represent significant differences in AMF colonization between inoculation levels while * indicates a significant difference in AMF colonization between $B t 11$ or $P$ plants within each spore treatment. 
a) Shoot biomass

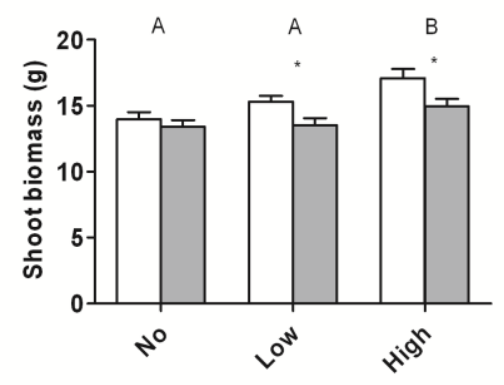

c) Root biomass

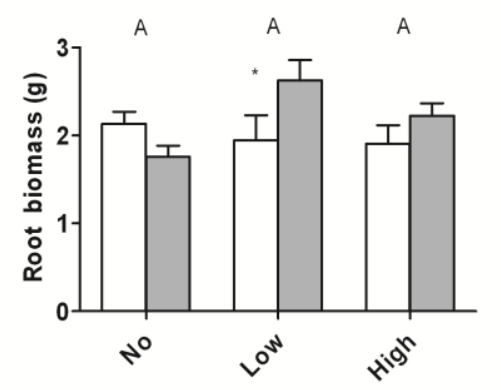

b) Chlorophyll content

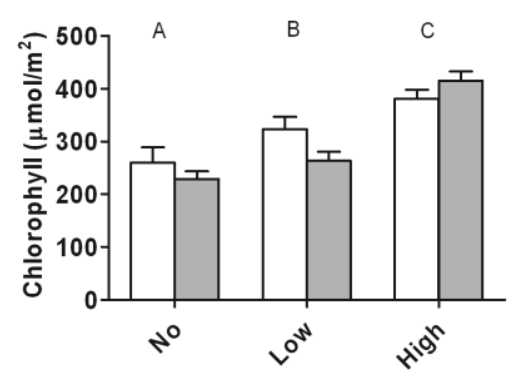

d) Root/shoot ratio

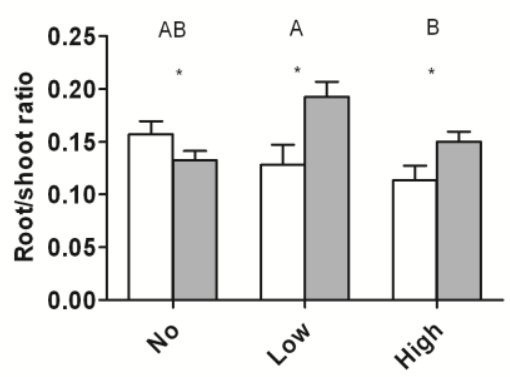

Figure 3. Effect of fertilizer treatment and plant type (Bt or P) on plant growth.

(a) shoot biomass, (b) chlorophyll content, (c) root biomass, and (d) root/shoot ratio of $B t 11$ and $P$ maize plants grown for 60 days with weekly treatments of 'No' $\left(0 \mathrm{~g} \mathrm{~L}^{-1}\right)$, ' $\mathrm{Low}^{\prime}\left(0.23 \mathrm{~g} \mathrm{~L}^{-1}\right)$, or 'High' $\left(1.87 \mathrm{~g} \mathrm{~L}^{-1}\right)$ fertilizer. Here, data from $B t 11$ and $P$ plants in each spore treatment were pooled to determine the overall effect of fertilizer level on growth. Open bars represent the means $( \pm \mathrm{SE})$ of transgenic $B t 11$ plants and solid bars represent the means $( \pm S E)$ of $P$ parental plants. $* P<0.05 ; n=$ 15 for each bar. Uppercase letters reflect the results of the Tukey multiple range test; means with a different letter represent significant differences in plant growth responses between fertilizer treatments while * indicates a significant difference in growth responses between $B t 11$ or $P$ plants within each fertilizer treatment. 
a) No fertilizer

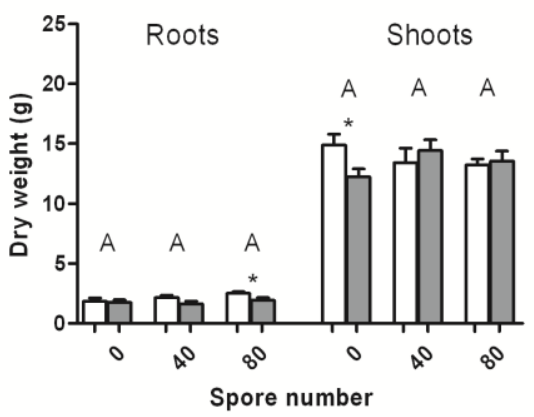

b) Low fertilizer

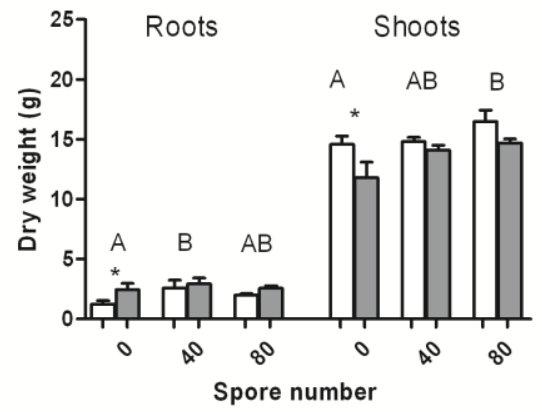

c) High fertilizer

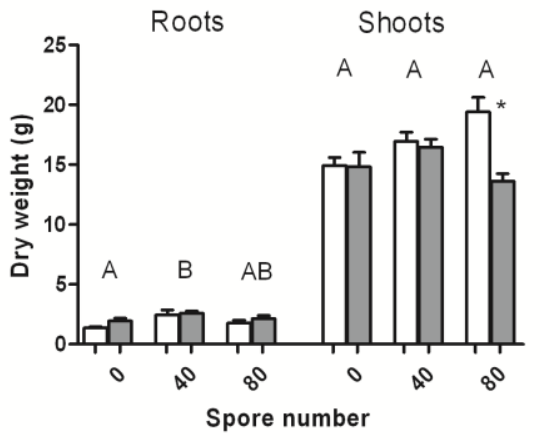

Figure 4. Effect of fertilizer treatment, spore density, and plant type on growth.

Root and shoot biomass of $B t 11$ and $P$ maize plants inoculated with 0,40 , or 80 spores of the AMF species Glomus mosseae and grown in the greenhouse for 60 days with weekly treatments of (a) 'No' $\left(0 \mathrm{~g} \mathrm{~L}^{-1}\right)$, (b) 'Low' $\left(0.23 \mathrm{~g} \mathrm{~L}^{-1}\right)$, or (c) 'High' $\left(1.87 \mathrm{~g} \mathrm{~L}^{-1}\right)$ fertilizer. Here, the effects of each treatment - plant type (Bt 11 or P), fertilizer level ('No', 'Low', or 'High') and spore inoculation level (0, 40, or 80) can be observed on the root and shoot biomass at the end of the experiment. Open bars represent the means $( \pm$ SE) of transgenic $B t 11$ plants and solid bars represent the means $( \pm S E)$ of $P$ parental plants. $* \mathbf{P}<0.05 ; n=5$ for each bar. Uppercase letters reflect the results of the Tukey multiple range 
test; means with a different letter represent significant differences in root and shoot dry weight between inoculation levels while * indicates a significant difference in dry weight between $B t 11$ or $\mathbf{P}$ plants within each spore treatment. 

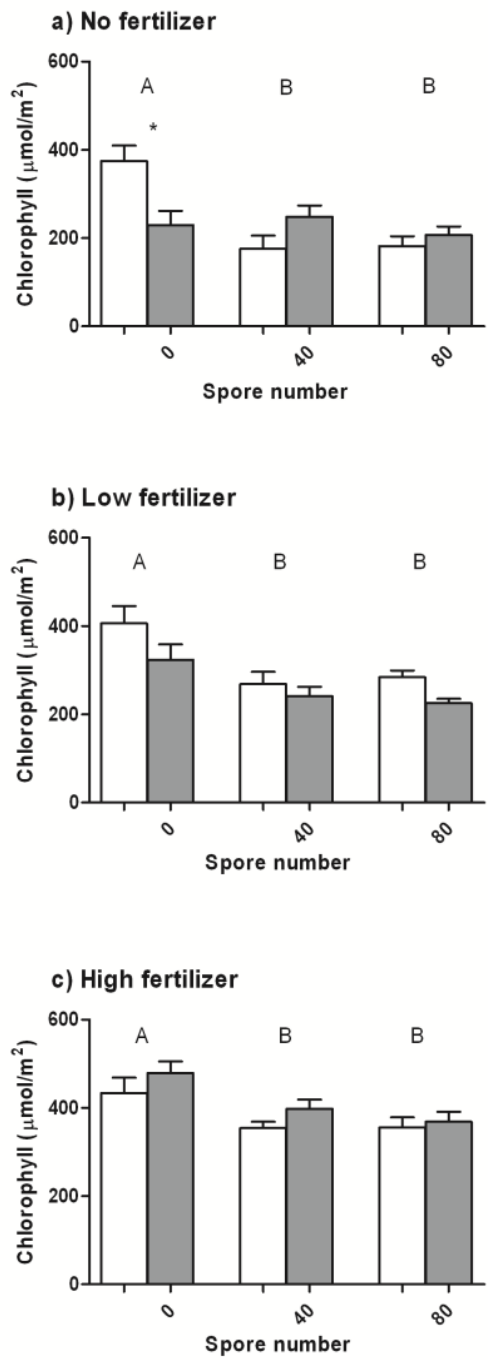

Figure 5. Effect of fertilizer, spore density, and plant type on leaf chlorophyll.

Chlorophyll content in leaf samples of $B t 11$ and $P$ maize plants inoculated with 0,40 , or 80 spores of the AMF species Glomus mosseae and grown in the greenhouse for 60 days with weekly treatments of (a) 'No' $\left(0 \mathrm{~g} \mathrm{~L}^{-1}\right)$, (b) 'Low' $\left(0.23 \mathrm{~g} \mathrm{~L}^{-1}\right)$, or (c) 'High' $\left(1.87 \mathrm{~g} \mathrm{~L}^{-1}\right)$ fertilizer. Here, the effects of each treatment - plant type, fertilizer level, and spore inoculation level - can be seen on chlorophyll content 60 days after inoculation. Open bars represent the means $( \pm \mathrm{SE})$ of transgenic $B t 11$ plants and solid bars represent the means $( \pm$ SE) of $P$ parental plants. $* P<0.05 ; n=5$ for each bar.

Uppercase letters reflect the results of the Tukey multiple range test; means with a different letter represent significant differences in chlorophyll content between spore inoculation levels while * indicates a significant difference in chlorophyll content between $B t 11$ or $P$ plants within each spore treatment. 


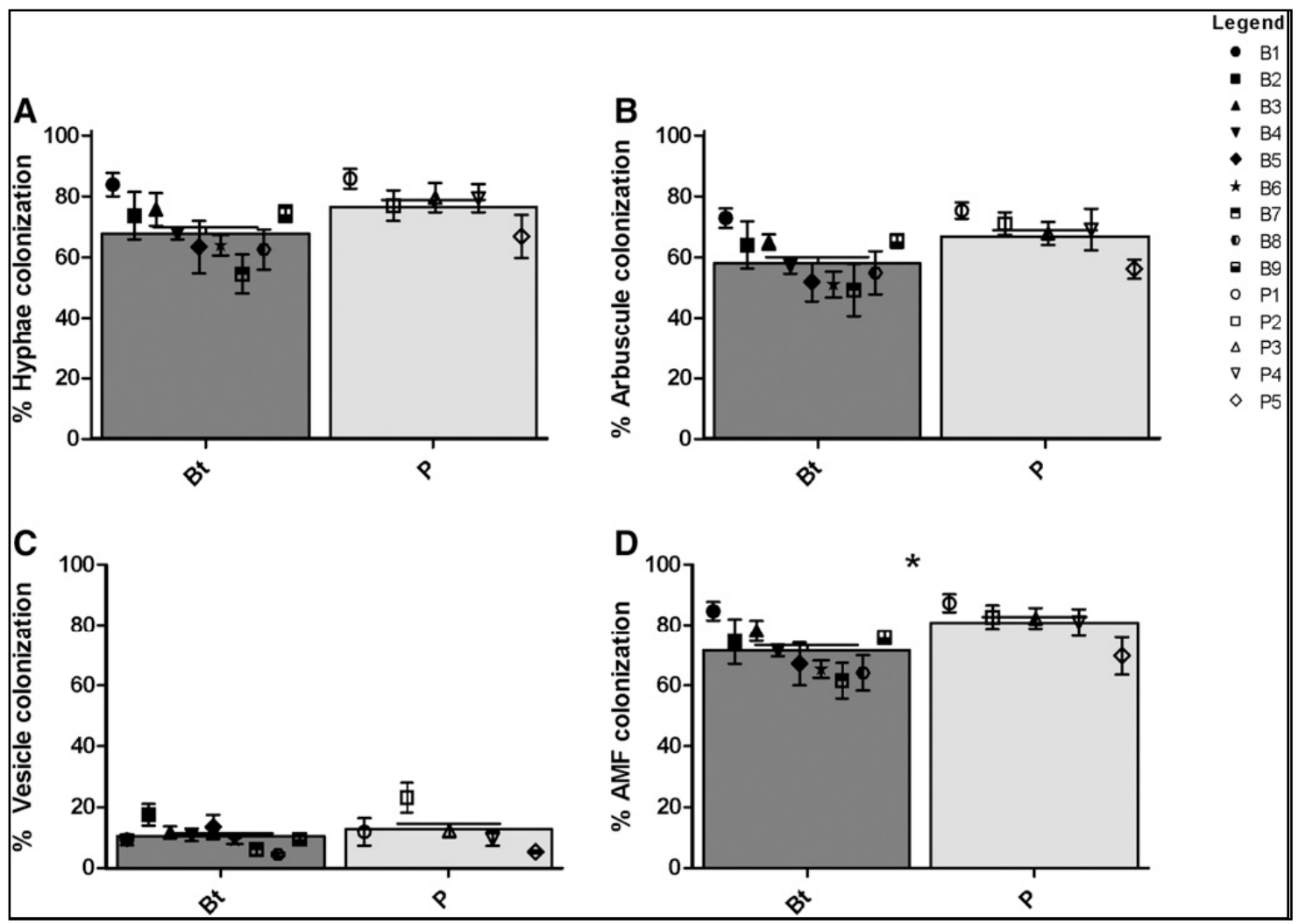

Figure 6. Effect of plant type (Bt or $\mathrm{P})$ on AMF in a 60 day greenhouse study.

Mean percentage incidence $( \pm \mathrm{SE})$ of $(\mathrm{A})$ AMF hyphal colonization, $(B)$ arbuscule colonization, $(C)$ vesicle colonization, and total percentage of (D) AMF colonization (per 100 intersects on root sample) in $B t$ and non-Bt parental (P) maize plants grown for 60 days in a greenhouse in $50 \%$ locallycollected agricultural soil. Dark gray bars represent the means $( \pm \mathrm{SE})$ of the pooled $B t$ AMF data $(\mathrm{N}$ $=45$ ); light gray bars represent the means $( \pm \mathrm{SE})$ of the pooled $P$ AMF data $(N=25) ; * P \leq 0.05$. Symbols represent means $( \pm \mathrm{SE})$ of the individual $B t$ and $P$ maize lines; $N=5$ for each symbol. P1: base-parental for B1, P2: parental for B2 and B5, P3: parental for B3 and B6, P4: parental for B4, P5: parental for $\mathrm{B} 7, \mathrm{B8}$, and $\mathrm{B9}$. 


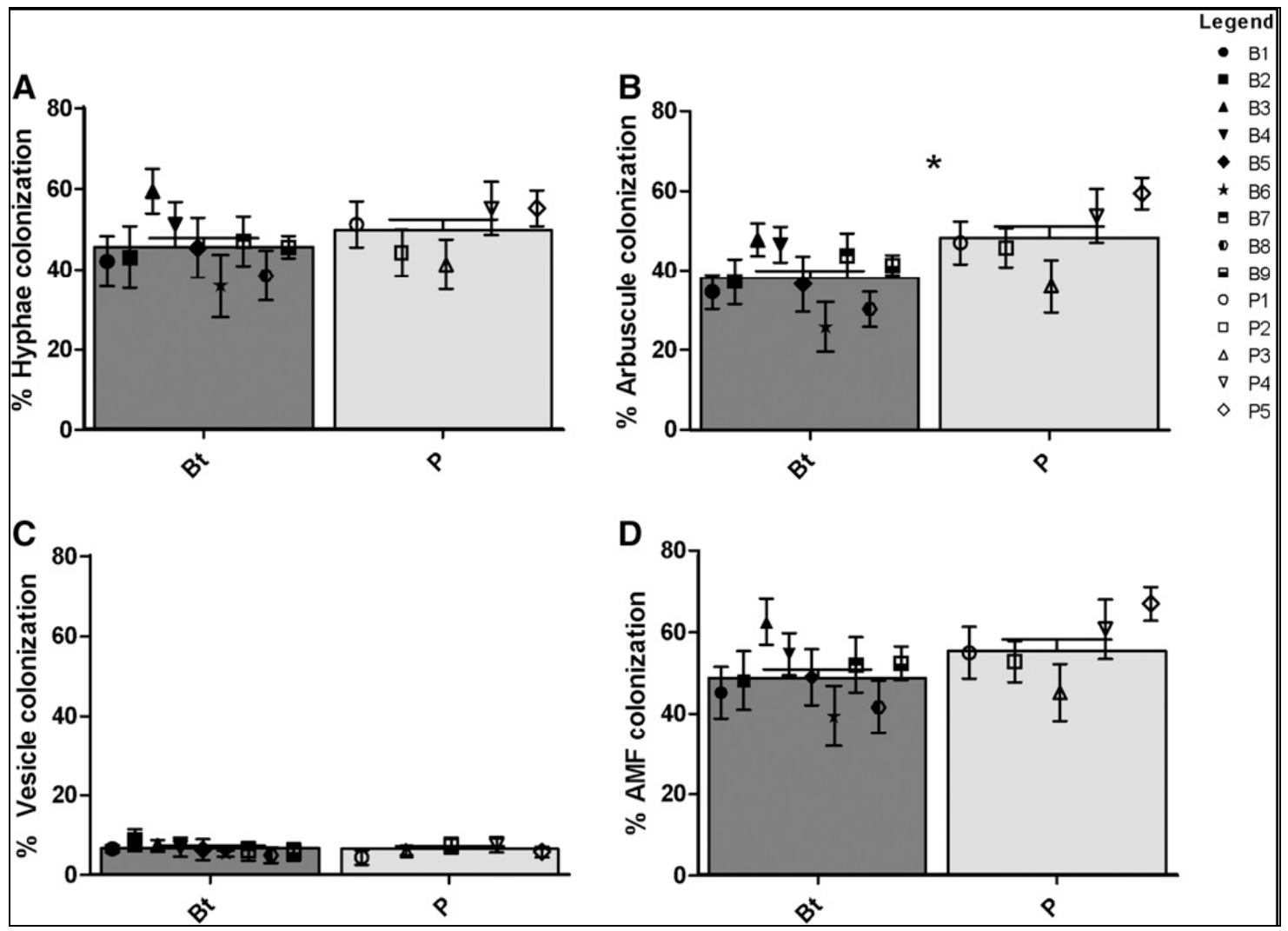

Figure 7. Effect of plant type (Bt or $P$ ) on AMF in a 100 day greenhouse study.

Mean percentage incidence ( \pm SE) of (A) AMF hyphal colonization, $(B)$ arbuscule colonization, $(C)$ vesicle colonization, and total percentage of (D) AMF colonization (per 100 intersects on root sample) in $B t$ and non- $B t$ parental (P) maize plants grown for 100 days in a greenhouse in $50 \%$ locallycollected agricultural soil. Dark gray bars represent the means $( \pm \mathrm{SE})$ of the pooled $B t$ AMF data (N $=45$ ); light gray bars represent the means $( \pm S E)$ of the pooled $P$ AMF data $(N=25) ; * P \leq 0.05$. Symbols represent means ( \pm SE) of the individual $B t$ and $P$ maize lines; $N=5$ for each symbol. $P 1$ : base-parental for B1, P2: parental for B2 and B5, P3: parental for B3 and B6, P4: parental for B4, P5: parental for $\mathrm{B} 7, \mathrm{B8}$, and $\mathrm{B9}$. 


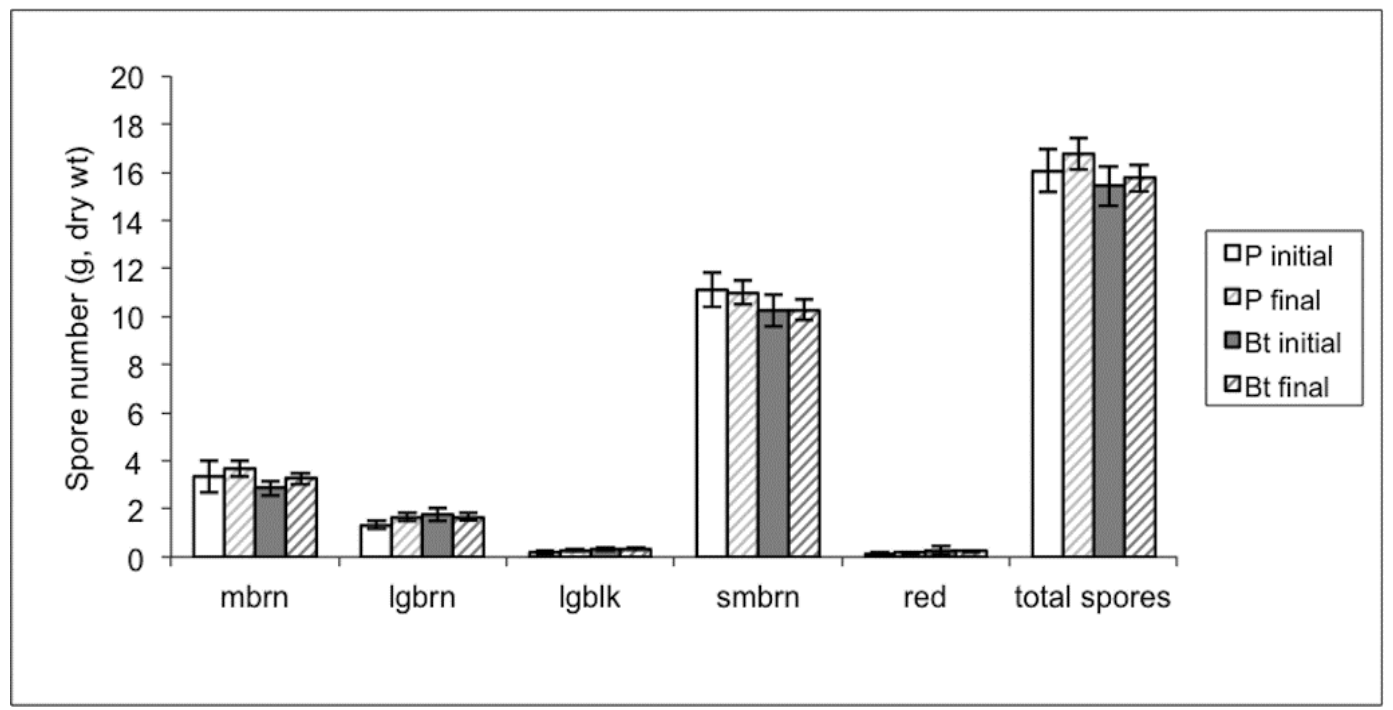

Figure 8. Effect of plant type (Bt or $\mathrm{P}$ ) on spore density in field plots.

Spores per gram of dry soil in soil samples collected from each $B t$ and P field plot in May 2009 (initial) and October 2009 (final). Five initial soil samples were collected from each plot and pooled for the spore extraction to determine initial spore abundance and diversity per plot before seeding. Spores were categorized into five morphological groups (medium brown, large brown, large black, small brown, and red) and total spores per gram dry soil were calculated. Five final soil samples were collected from each plot at the end of the field season and spores were extracted from five soil samples per plot to determine whether $B t$ maize had a negative effect on spore abundance and diversity after one growing season. Open bars represent the means $( \pm$ SE) of spore counts from initial soil samples collected from $P$ plots and solid bars represent the means $( \pm$ SE) of initial spore counts from $B t$ plots; hatched lines represent final spore counts collected from $P$ plots (light gray lines) and $B t$ plots (dark gray lines). $\mathbf{n}=10$ for $P$ initial, $n=14$ for $B t$ initial, $n=50$ for $P$ final, and $n=70$ for $B t$ final. 


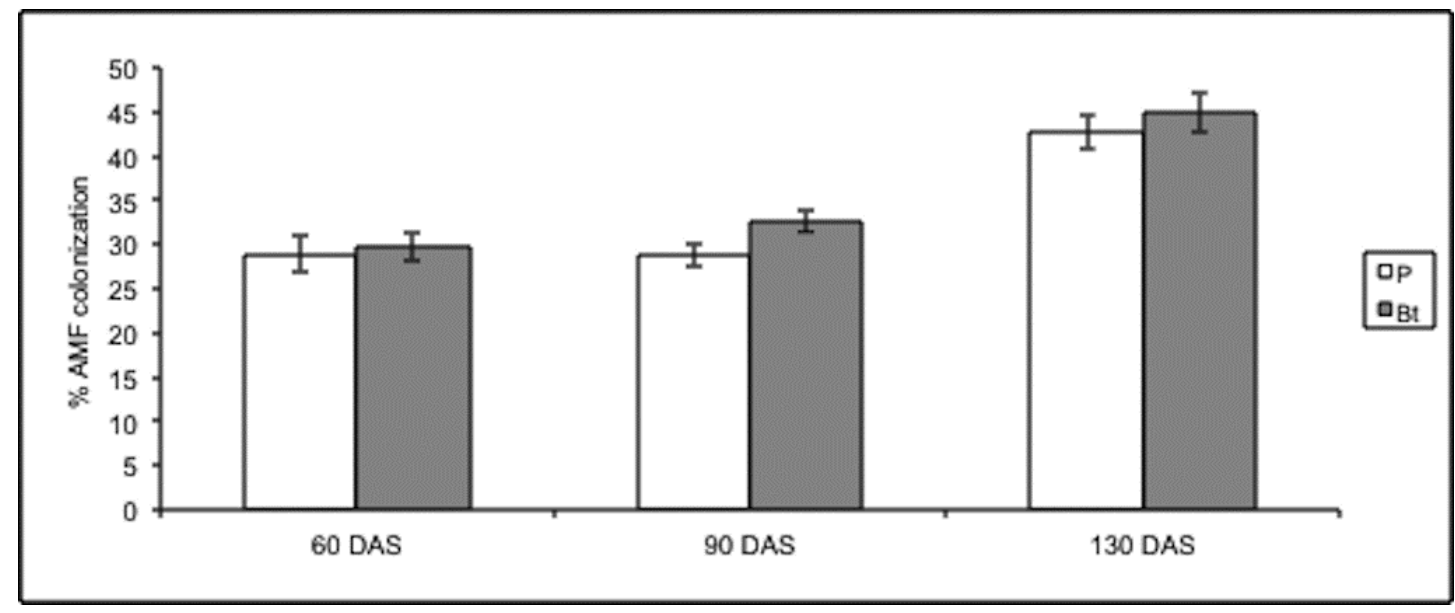

Figure 9. Percent AMF colonization in $B t$ and $P$ maize in a field experiment.

Percent AMF colonization of non-Bt parental (P) and $B t$ maize roots 60, 90, and 130 day after sowing (DAS). Open bars represent the means $( \pm \mathrm{SE})$ of non-Bt parental maize lines and solid bars represent the means $( \pm \mathrm{SE}$ ) of $B t$ maize lines. Five plants were harvested from each plot 60 DAS, 10 plants were harvested from each plot 90 DAS, and 5 plants were harvested from each plot 130 DAS, for a total of 480 root samples over the course of this experiment. 


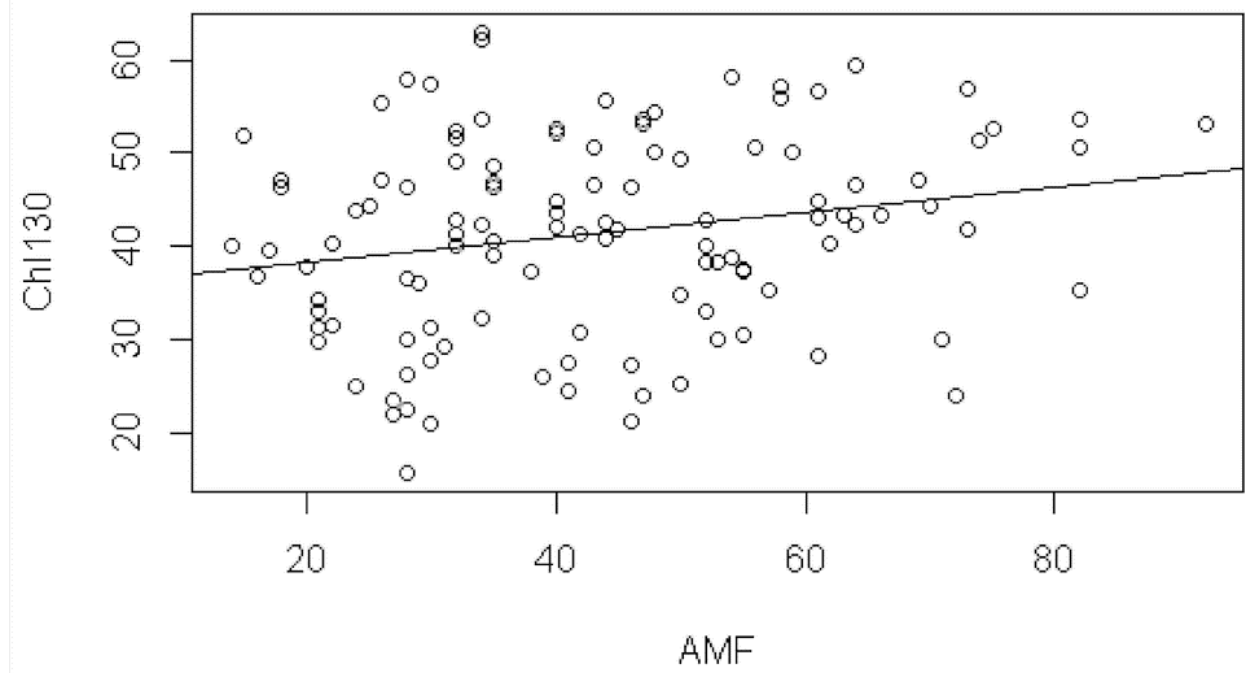

Figure 10. Correlation between leaf chlorophyll content and AMF at $130 \mathrm{~d}$.

Leaf chlorophyll content was positively correlated with percentage AMF colonization of roots at the 130 day harvest in a field experiment. Pearson correlation coefficient $=0.22, P=0.02$; Proc mixed $F_{1,90}=4.61, P=0.03$. Leaf chlorophyll content was assessed on 5 plants per plot $130 \mathrm{~d}$ after sowing for a total of 120 leaf chlorophyll measurements. 


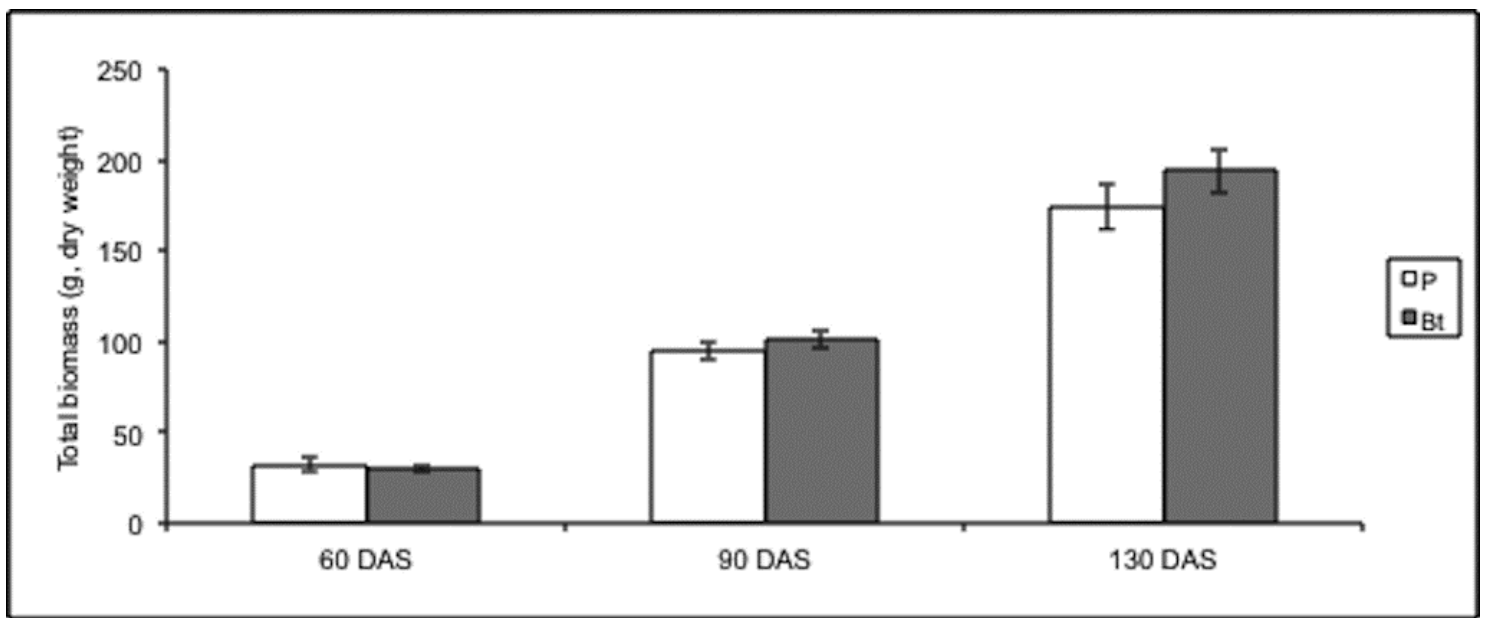

Figure 11. Biomass of $B t$ and $P$ maize 60, 90, and 130 days after sowing in a field.

Total biomass (roots + shoots + ears) of non-Bt parental $(P)$ and $B t$ maize 60, 90, and 130 days after sowing (DAS) in a field experiment. Open bars represent the means $( \pm$ SE) of non-Bt parental maize lines and solid bars represent the means $( \pm \mathrm{SE})$ of $B t$ maize lines. From each plot, five plants were harvested $60 \mathrm{DAS}, 10$ plants were harvested $90 \mathrm{DAS}$, and 5 plants were harvested $130 \mathrm{DAS}$, for a total of 480 plants over the course of the experiment. 


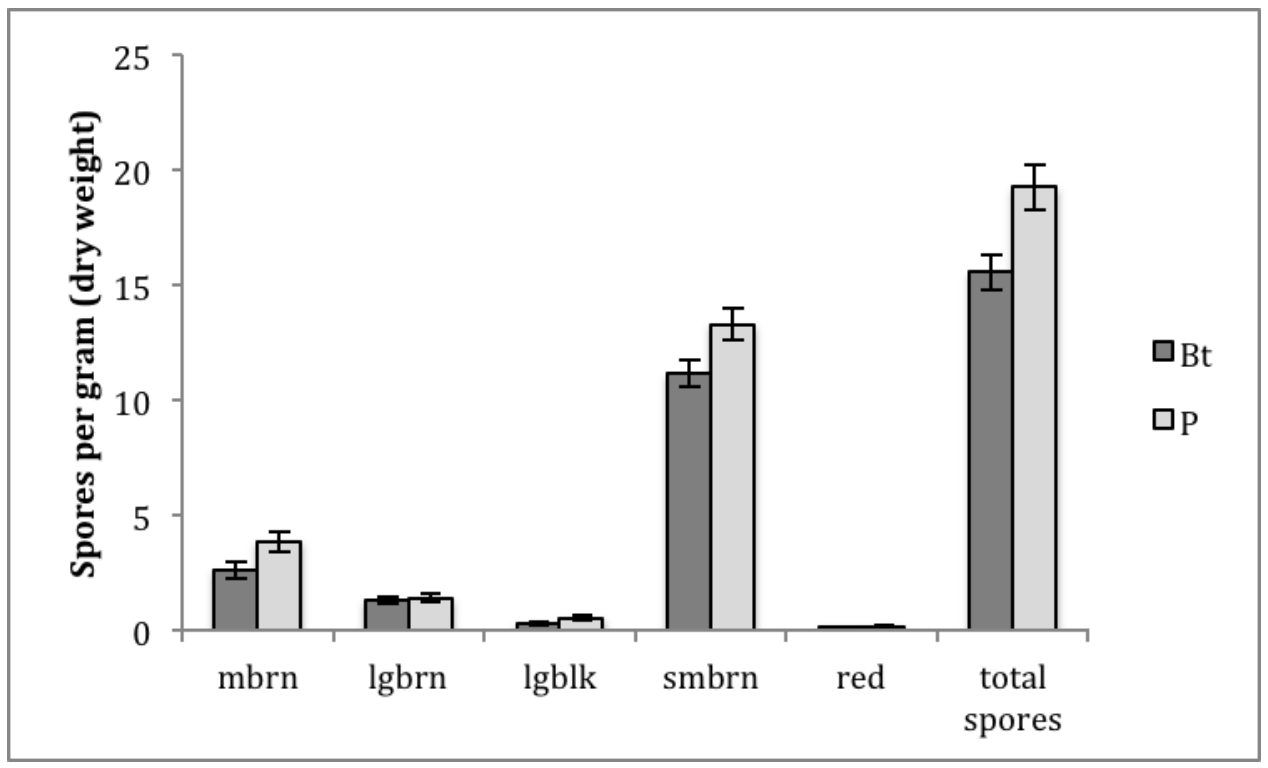

Figure 12. Spore density in $B t$ and $P$ plots as affected by cultivation history.

Spores per gram of dry soil collected from $B t$ and parental (P) maize field plots in May 2010. Three soil samples from each plot were used to determine initial spore abundance and diversity as affected by $B t$ or non-Bt plot history. Dark gray bars represent means (+/-SE) of spores collected from plots with a $B t$ history $(n=42$ soil samples for each bar); light gray bars represent means $(+/$-SE) of spores collected from plots with a $P$ history $(n=30$ soil samples for each bar). Spores were categorized into five morphological groups (medium brown, large brown, large black, small brown, and red) and total spores per gram dry soil were calculated. 
a) Hyphae

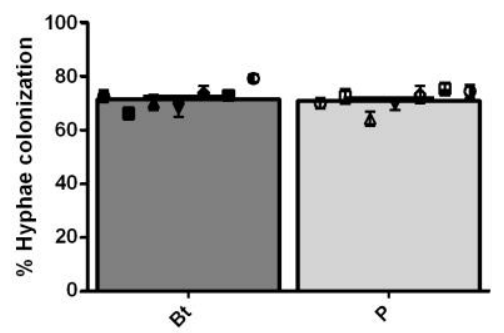

c) Vesicles

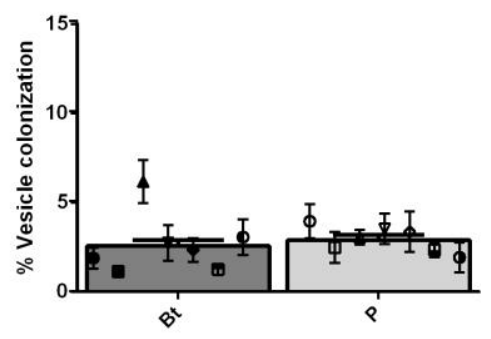

b) Arbuscules

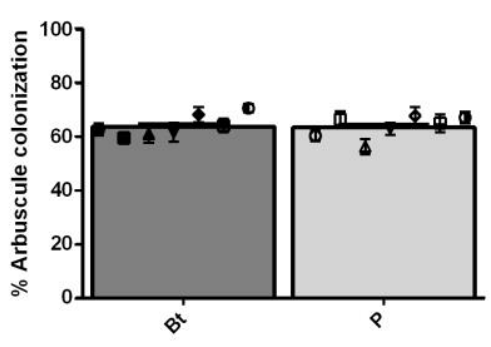

d) Total AMF

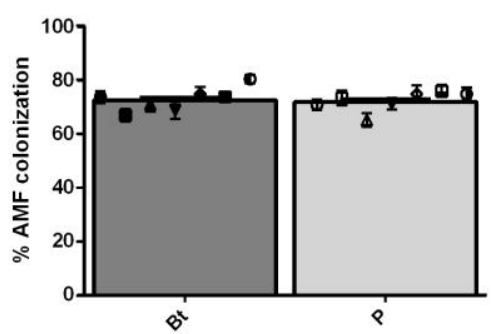

Figure 13. AMF colonization in $B t$ and $P$ maize in split-plots in a field experiment.

Mean percent (+/- SE) of (a) AMF hyphal colonization, (b) arbuscule colonization, (c) vesicle colonization, and (d) total percent AMF colonization (per 100 intersects) in $B t$ and non-Bt parental (P) maize plants grown for 60 days in split-plots in a field experiment. Dark gray bars represent means (+/-SE) of pooled $B t$ AMF data ( $n=167$ plants for each bar); light gray bars represent means (+/-SE) of pooled P AMF data ( $n=165$ plants for each bar). Symbols represent means (+/- SE) of the individual $B t$ and $P$ maize genotypes; $n=24$ plants for each symbol. 


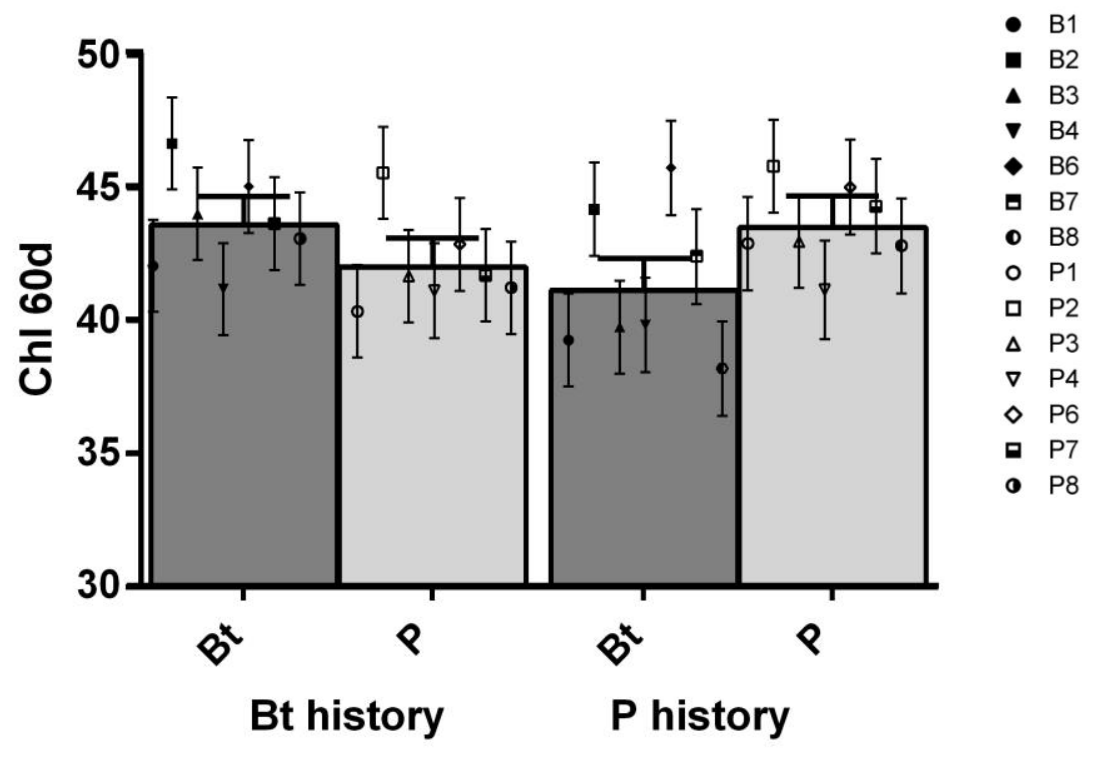

Figure 14. Positive feedback fitness effects as affected by plot cultivation history.

Mean leaf chlorophyll content (+/- SE) in $B t$ and non-Bt parental (P) maize plants grown in split-plots in a 60-day field experiment. Dark gray bars represent means (+/-SE) of leaf chlorophyll content in $B t$ plants grown in plots with a $B t$ cultivation history (left; $\mathbf{n = 8 4} B t$ plants grown in $B t$ history) or a non- $B t$ parental cultivation history (right; $n=59 B t$ plants grown in $P$ history); light gray bars represent means (+/-SE) of leaf chlorophyll content in $P$ plants grown in plots with a $B t$ cultivation history (left; $\mathbf{n}=84 \mathrm{P}$ plants grown in $B t$ history) or a non-Bt cultivation history (right; $\mathbf{n}=58 \mathrm{P}$ plants grown in $P$ history). Symbols represent means $(+/-\mathrm{SE})$ of the individual $B t$ and $P$ maize genotypes grown in each plot; $n=12$ plants for each symbol in plots with a $B t$ history, $n=5$ to 12 in plots with a $P$ history, depending on the plot. 


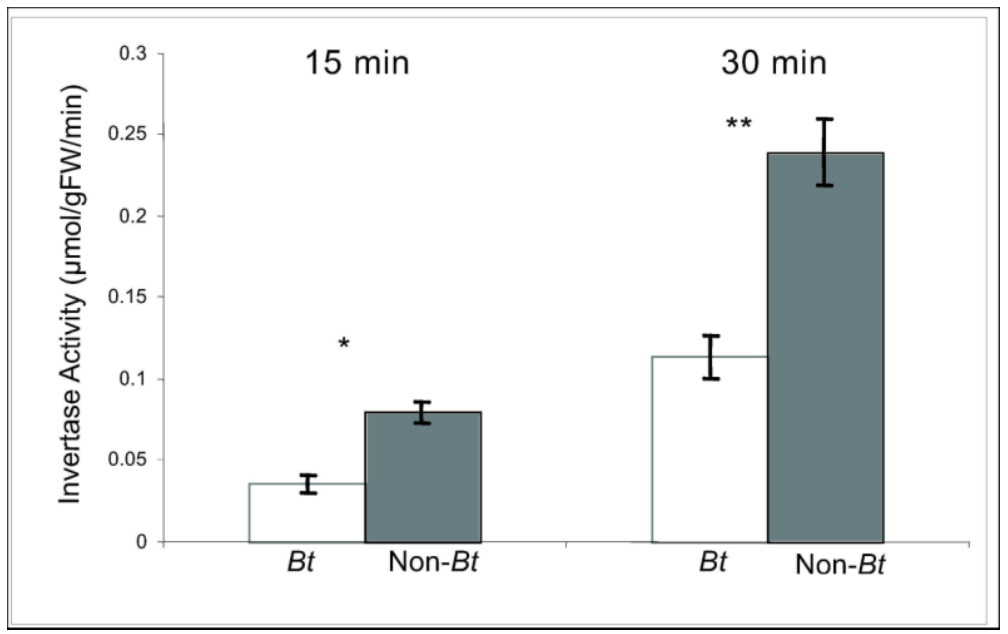

Figure 15. Apoplastic invertase activity in $B t 11$ and $P$ maize roots.

Acid invertase activity in $B t$ (open bars) and non-Bt (solid) maize roots in vivo using the DNS method. Invertase activity in the roots is expressed as $\mu \mathrm{mol}$ of reducing sugar released per gram of fresh root weight per minute. Incubation times were 15 and 30 minutes. Bars represent means $\pm S E$. $* P=$ $0.0001 ; n=8$. $* * \mathbf{P}=0.0005 ; \mathrm{n}=8$. 


\begin{tabular}{|c|c|c|c|}
\hline$\underline{\text { Stain }}$ & $\frac{\text { Material }}{\text { detected }}$ & $\underline{B t}$ & $\underline{\mathbf{P}}$ \\
\hline $\begin{array}{l}\text { Toluidine } \\
\text { Blue }\end{array}$ & $\begin{array}{l}\text { Differences in } \\
\text { cell wall } \\
\text { layers; } \\
\text { phenolics; } \\
\text { liginified } \\
\text { structures stain } \\
\text { blue }\end{array}$ & & \\
\hline $\begin{array}{l}\text { Safranin } \\
\text { O }\end{array}$ & Lignin & & \\
\hline $\begin{array}{l}\text { Berberine } \\
\text { Hemi- } \\
\text { Sulfate + } \\
\text { Analine } \\
\text { Blue }\end{array}$ & $\begin{array}{l}\text { Suberin and } \\
\text { lignin }\end{array}$ & & \\
\hline $\begin{array}{l}\text { Sudan } \\
\text { Red III }\end{array}$ & Suberin & & \\
\hline
\end{tabular}




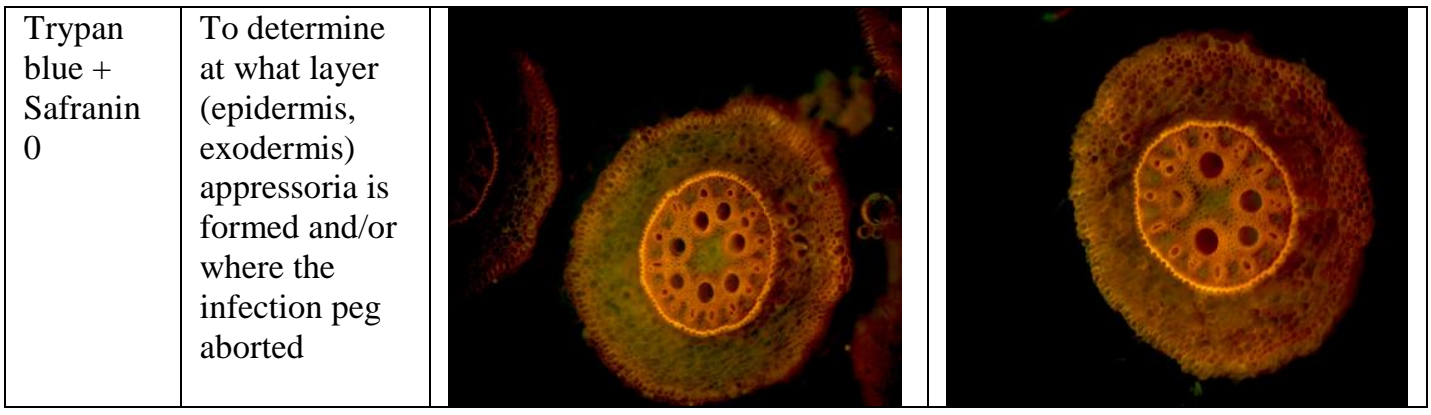

Figure 16. Stained cross-sections of $B t$ and non-Bt maize roots.

Fresh cut cross-sections of $B t 11$ (Attribute) and non-Bt maize (Providence) roots stained for 3-5 minutes in Toluidine blue, Safranin O, Berberine Hemi-Sulfate + Analine Blue, or Sudan Red III to visualize lignin and suberin content in epidermal and cortical cells. 

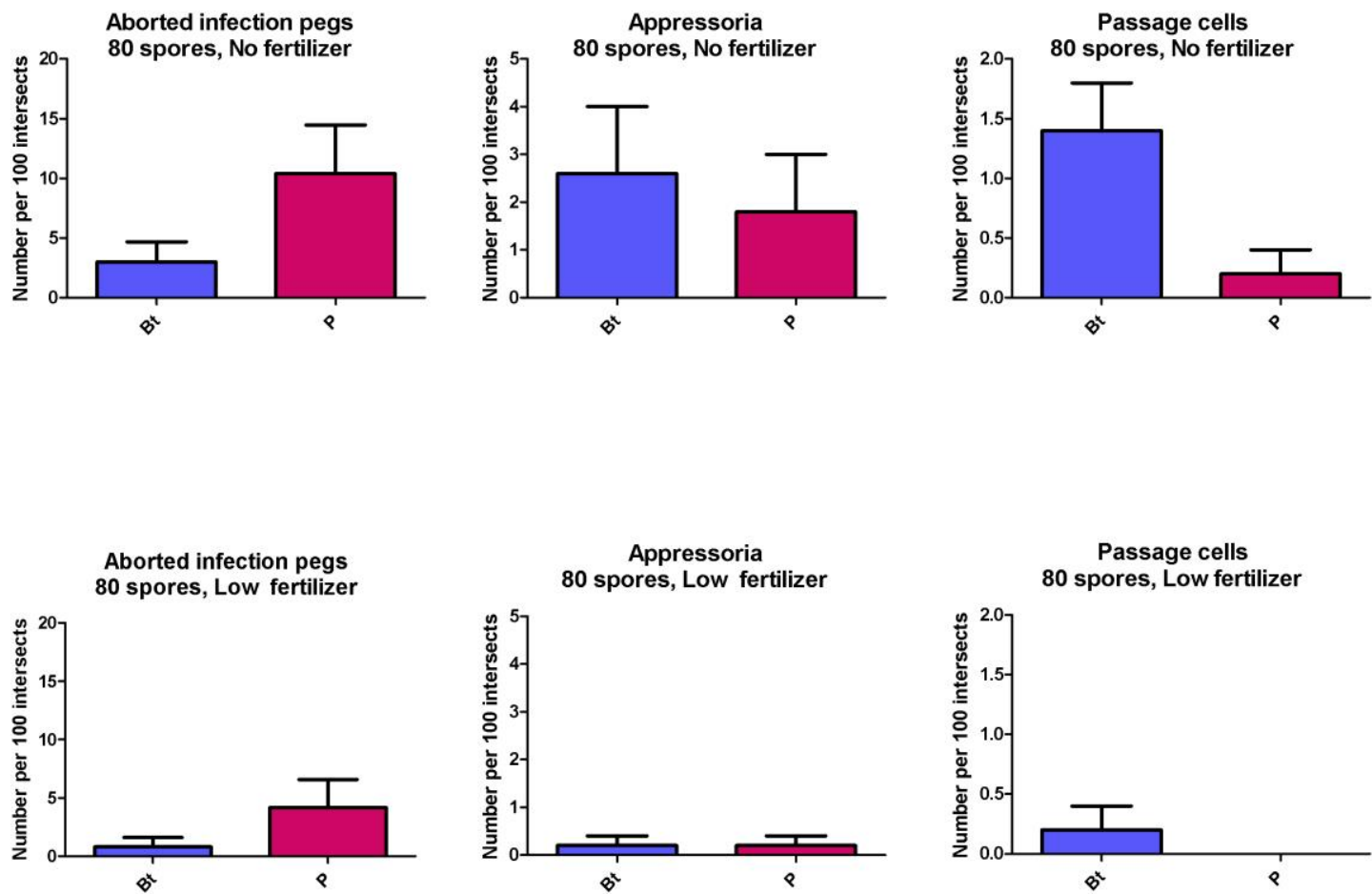

Figure 17. Potential pre-symboiotic barriers in $B t 11$ and non-Bt maize.

Mean number of aborted infection pegs, appressoria, and passage cells in $B t 11$ (Attribute) and non$B t$ parental (Providence; $\mathrm{P}$ ) maize plants grown for 60 days with weekly treatments of 'No' $\left(0 \mathrm{~g} \mathrm{~L}^{-1}\right)$ (top) or 'Low' $\left(0.23 \mathrm{~g} \mathrm{~L}^{-1}\right)$ (bottom) fertilizer in a greenhouse and incolualated with 80 spores of Glomus mosseae (Cheeke et al., 2011; Ch. 2). Blue bars represent means (+/-SE) of aborted infection pegs, appressoria, and passage cells in $B t$ plants; red bars represent means (+/-SE) of aborted infection pegs, appressoria, and passage cells in $P$ plants. $n=5$ plants for each bar. 


\section{References}

\section{Chapter 1. References}

Abel, C. A. and J. J. Adamczyk. 2004. Relative concentration of Cry1A in maize leaves and cotton bolls with diverse chlorophyll content and corresponding larval development of fall armyworm (Lepidoptera : Noctuidae) and southwestern corn borer (Lepidoptera : Crambidae) on maize whorl leaf profiles. Journal of Economic Entomology 97:1737-1744.

Adamczyk, J. J. and D. D. Hardee. 2002. Insect-resistant transgenic crops. Crop Biotechnology 829:23-37.

Ahl Goy, P., G. Warren, J. White, L. Pivalle, P. L. Fearing, and D. Vlachos. 1995. Interaction of insect tolerant maize with organisms in the ecosystem. Mitteilungen des Biologischen Bundesamts fur Forst- und Landwirschaft 309:50-53.

Ahmad, A., G. E. Wilde, and K. Y. Zhu. 2006. Evaluation of effects of Coleopteranspecific Cry3Bb1 protein on earthworms exposed to soil containing corn roots or biomass. Environmental Entomology 35:976-985.

Al-Deeb, M. A., G. E. Wilde, J. M. Blair, and T. C. Todd. 2003. Effect of Bt corn for corn rootworm control on nontarget soil microarthropods and nematodes. Environmental Entomology 32:859-865.

Alstad, D. N. and D. A. Andow. 1996. Implementing management of insect resistance to transgenic crops. AgBiotech News and Information 8:177-181.

Anderson, P. L., R. L. Hellmich, M. K. Sears, D. V. Sumerford, and L. C. Lewis. 2004. Effects of Cry1Ab-expressing corn anthers on monarch butterfly larvae. Environmental Entomology 33:1109-1115.

Anderson, P. L., R. L. Hellmich, J. R. Prasifka, and L. C. Lewis. 2005. Effects on fitness and behavior of monarch butterfly larvae exposed to a combination of Cry1 Abexpressing corn anthers and pollen. Environmental Entomology 34:944-952.

Bais, H. P., T. L. Weir, L. G. Perry, S. Gilroy, and J. M. Vivanco. 2006. The role of root exudates in rhizosphere interations with plants and other organisms. Annual Review of Plant Biology 57:233-266.

Bakonyi, G., F. Szira, I. Kiss, I. Villanyi, A. Seres, and A. Szekacs. 2006. Preference tests with collembolas on isogenic and Bt-maize. European Journal of Soil Biology 42:S132-S135.

Bakonyi, G., A. Dolezsai, N. Matrai, and A. Szekacs. 2011. Effects of consumption of Btmaize (MON 810) on the Collembolan Folsomia candida, over multiple generations: A laboratory study. Insects 2:243-252.

Baumgarte, S. and C. C. Tebbe. 2005. Field studies on the environmental fate of the Cry1Ab Bt-toxin produced by transgenic maize (MON810) and its effect on bacterial communities in the maize rhizosphere. Molecular Ecology 14:2539- 
2551

Beegle, C. C. and T. Yamamoto. 1992. Invitation paper (CP-Alexander-Fund) - History of Bacillus-thuringiensis Berliner Reseach-and-Development. Canadian Entomologist 124:587-616.

Bhatti, M. A., J. Duan, G. Head, C. J. Jiang, M. J. McKee, T. E. Nickson, C. L. Pilcher, and C. D. Pilcher. 2005. Field evaluation of the impact of corn rootworm (Coleoptera : Chrysomelidae)-protected Bt corn on ground-dwelling invertebrates. Environmental Entomology 34:1325-1335.

Birch, A. N. E., B. S. Griffiths, S. Caul, J. Thompson, L. H. Heckmann, P. H. Krogh, and J. Cortet. 2007. The role of laboratory, glasshouse and field scale experiments in understanding the interactions between genetically modified crops and soil ecosystems: A review of the ECOGEN project. Pedobiologia 51:251-260.

Blackwood, C. B. and J. S. Buyer. 2004. Soil microbial communities associated with $B t$ and non-Bt corn in three soils. Journal of Environmental Quality 33:832-836.

Blair, J. M., P. J. Bohlen, and D. W. Freckman. 1996. Soil invertebrates as indicators of soil quality. In Methods for Assessing Soil Quality, eds. J. W. Doran and A. J. Jones, 283-301. Madison, WI: Soil Science Society of America.

Bravo, A., S. S. Gill, and M. Soberon. 2007. Mode of action of Bacillus thuringiensis Cry and Cyt toxins and their potential for insect control. Toxicon 49:423-435.

Broderick, N. A., K. F. Raffa, and J. Handelsman. 2006. Midgut bacteria required for Bacillus thuringiensis insecticidal activity. Proceedings of the National Academy of Sciences of the United States of America 103:15196-15199.

Broderick, N. A., C. J. Robinson, M. D. McMahon, J. Holt, J. Handelsman, and K. F. Raffa. 2009. Contributions of gut bacteria to Bacillus thuringiensis-induced mortality vary across a range of Lepidoptera. BMC Biology 7:11.

Brusetti, L., P. Francia, C. Bertolini, A. Pagliuca, S. Borin, C. Sorlini, A. Abruzzese, G. Sacchi, C. Viti, L. Giovannetti, E. Giuntini, M. Bazzicalupo, and D. Daffonchio. 2004. Bacterial communities associated with the rhizosphere of transgenic Bt 176 maize (Zea mays) and its non transgenic counterpart. Plant and Soil 266:11-21.

Candolfi, M. P., K. Brown, C. Grimm, B. Reber, and H. Schmidli. 2004. A faunistic approach to assess potential side-effects of genetically modified Bt-corn on nontarget arthropods under field conditions. Biocontrol Science and Technology 14:129-170.

Castaldini, M., A. Turrini, C. Sbrana, A. Benedetti, M. Marchionni, S. Mocali, A. Fabiani, S. Landi, F. Santomassimo, B. Pietrangeli, M. P. Nuti, N. Miclaus, and M. Giovannetti. 2005. Impact of Bt corn on rhizospheric and soil eubacterial communities and on beneficial mycorrhizal symbiosis in experimental microcosms. Applied and Environmental Microbiology 71:6719-6729.

Chambers, C. P., M. R. Whiles, E. J. Rosi-Marshall, J. L. Tank, T. V. Royer, N. A. Griffiths, M. A. Evans-White, and A. R. Stojak. 2010. Responses of stream 
macroinvertebrates to $B t$ maize leaf detritus. Ecological Applications 20:19491960.

Cheeke, T. E., B. A. Pace, T. N. Rosenstiel, and M. B. Cruzan. 2011. The influence of fertilizer level and spore density on arbuscular mycorrhizal colonization of transgenic Bt 11 maize (Zea mays) in experimental microcosms. FEMS Microbiology Ecology 75:304-312.

Cheeke, T. E., T. N. Rosenstiel, and M. B. Cruzan. in prep. Transgenes in maize: Evidence of reduced arbuscular mycorrhizal fungi colonization in multiple $B t$ maize lines.

Chen, Z. H. C. Z. H., L. J. Chen, Y. L. Zhang, and Z. J. Wu. 2011. Microbial properties, enzyme activities and the persistence of exogenous proteins in soil under consecutive cultivation of transgenic cottons (Gossypium hirsutum L.). Plant Soil and Environment 57:67-74.

Clark, B. W., T. A. Phillips, and J. R. Coats. 2005. Environmental fate and effects of Bacillus thuringiensis (Bt) proteins from transgenic crops: A review. Journal of Agricultural and Food Chemistry 53:4643-4653.

Clark, B. W. and J. R. Coats. 2006. Subacute effects of CrylAb Bt corn litter on the earthworm Eisenia fetida and the springtail Folsomia candida. Environmental Entomology 35:1121-1129.

Clark, B. W., K. R. Prihoda, and J. R. Coats. 2006. Subacute effects of transgenic Cry1Ab Bacillus thuringiensis corn litter on the isopods Trachelipus rathkii and Armadillidium nasatum. Environmental Toxicology and Chemistry 25:2653-2661.

Cortet, J., B. S. Griffiths, M. Bohanec, D. Demsar, M. N. Andersen, S. Caul, A. N. E. Birch, C. Pernin, E. Tabone, A. de Vaufleury, X. Ke, and P. H. Krogh. 2007. Evaluation of effects of transgenic $B t$ maize on microarthropods in a European multi-site experiment. Pedobiologia 51:207-218.

Crecchio, C. and G. Stotzky. 1998. Insecticidal activity and biodegradation of the toxin from Bacillus thuringiensis subsp. kurstaki bound to humic acids from soil. Soil Biology and Biochemistry 30:463-470.

de Vaufleury, A., P. E. Kramarz, P. Binet, J. Cortet, S. Caul, M. N. Andersen, E. Plumey, M. Coeurdassier, and P. H. Krogh. 2007. Exposure and effects assessments of Btmaize on nontarget organisms (gastropods, microarthropods, mycorrhizal fungi) in microcosms. Pedobiologia 51:185-194.

Debeljak, M., J. Cortet, D. Demsar, P. H. Krogh, and S. Dzeroski. 2007. Hierarchical classification of environmental factors and agricultural practices affecting soil fauna under cropping systems using Bt maize. Pedobiologia 51:229-238.

Devare, M. H., C. M. Jones, and J. E. Thies. 2004. Effect of Cry3Bb transgenic corn and tefluthrin on the soil microbial community: Biomass, activity, and diversity. Journal of Environmental Quality 33:837-843.

Devare, M., L. M. Londono-R, and J. E. Thies. 2007. Neither transgenic Bt maize 
(MON863) nor tefluthrin insecticide adversely affect soil microbial activity or biomass: A 3-year field analysis. Soil Biology and Biochemistry 39:2038-2047.

Dively, G. P., R. Rose, M. K. Sears, R. L. Hellmich, D. E. Stanley-Horn, D. D. Calvin, J. M. Russo, and P. L. Anderson. 2004. Effects on monarch butterfly larvae (Lepidoptera : Danaidae) after continuous exposure to Cry1Ab-expressing corn during anthesis. Environmental Entomology 33:1116-1125.

Dobbelaere, S., J. Vanderleyden, and Y. Okon. 2003. Plant growth-promoting effects of diazotrophs in the rhizosphere. Critical Reviews in Plant Sciences 22:107-149.

Donegan, K. K., C. J. Palm, V. J. Fieland, L. A. Porteous, L. M. Ganio, D. L. Schaller, L. Q. Bucao, and R. J. Seidler. 1995. Changes in levels, species and DNA fingerprints of soil-microorganisms associated with cotton expressing the Bacillus thuringiensis var kurstaki endotoxin. Applied Soil Ecology 2:111-124.

Donegan, K. K., D. L. Schaller, J. K. Stone, L. M. Ganio, G. Reed, P. B. Hamm, and R. J. Seidler. 1996. Microbial populations, fungal species diversity and plant pathogen levels in field plots of potato plants expressing the Bacillus thuringiensis var tenebrionis endotoxin. Transgenic Research 5:25-35.

Douville, M., F. Gagne, C. Blaise, and C. Andre. 2007. Occurrence and persistence of Bacillus thuringiensis $(B t)$ and transgenic $B t$ corn Cry1 Ab gene from an aquatic environment. Ecotoxicology and Environmental Safety 66:195-203.

Duan, J. J., M. Marvier, J. Huesing, G. Dively, and Z. Y. Huang. 2008. A meta-analysis of effects of Bt crops on honey bees (Hymenoptera: Apidae). PloS One 3:e1415.

Dunfield, K. E. and J. J. Germida. 2003. Seasonal changes in the rhizosphere microbial communities associated with field-grown genetically modified canola (Brassica napus). Applied and Environmental Microbiology 69:7310-7318.

Dunfield, K. E. and J. J. Germida. 2004. Impact of genetically modified crops on soiland plant-associated microbial communities. Journal of Environmental Quality 33:806-815.

EPA, U. S. Environmental Protection Agency. 2001a. Amended Revised Response to EPA's Data Call-In Notice Concerning the Potential for Adverse Effects of Bt Corn on Nontarget Lepidopterans. http://www.epa.gov/oppbppd1/biopesticides/pips/executive_summary_and_prefac e.pdf

EPA, U. S. Environmental Protection Agency. 2001b. SAP Report No 2000-07, Sets of Scientific Issues being Considered by the Environmental Protection Agency Regarding: Bt Plant Pesticides Risk and Benefits Assessments. http://www.epa.gov/scipoly/sap/meetings/2000/october/octoberfinal.pdf.

EPA, U. S. Environmental Protection Agency. 2009. SmartStax Pesticide Fact Sheet. http://www.epa.gov/oppbppd1/biopesticides/pips/smartstax-factsheet.pdf

EPA, U. S. Environmental Protection Agency. 2010. Introduction to Biotechnology Regulation for Pesticides. 
http://www.epa.gov/oppbppd1/biopesticides/regtools/biotech-reg-prod.htm.

EPA, U. S. Environmental Protection Agency. 2011. Pesticides: Regulating

Biopesticides, Plant Incorporated Protectants, Current and Previously Registered Secion 3 PIP Registrations.

http://www.epa.gov/pesticides/biopesticides/pips/pip_list.htm.

Escher, N., B. Käch, and W. Nentwig. 2000. Decomposition of transgenic Bacillus thuringiensis maize by microorganisms and woodlice Porcellio scaber (Crustacea: Isopoda). Basic and Applied Ecology 1:161-169.

Fang, M., R. J. Kremer, P. P. Motavalli, and G. Davis. 2005. Bacterial diversity in rhizospheres of nontransgenic and transgenic corn. Applied and Environmental Microbiology 71:4132-4136.

Fang, M., P. P. Motavalli, R. J. Kremer, and K. A. Nelson. 2007. Assessing changes in soil microbial communities and carbon mineralization in $B t$ and non-Bt corn residue-amended soils. Applied Soil Ecology 37:150-160.

Federici, B. A. 2002. Case study: Bt crops, a novel mode of insect control. In Genetically Modified Crops: Assessing Safety, ed. K. T. Atherton, 164-200. New York, NY: Taylor and Francis Inc.

Ferreira, L., J. C. Molina, C. Brasil, and G. Andrade. 2003. Evaluation of Bacillus thuringiensis bioinsecticidal protein effects on soil microorganisms. Plant and Soil 256:161-168.

Flores, S., D. Saxena, and G. Stotzky. 2005. Transgenic Bt plants decompose less in soil than non-Bt plants. Soil Biology and Biochemistry 37:1073-1082.

Gao, Y. L., J. J. An, C. X. Liu, and K. M. Wu. 2010. Monitoring of Bt resistance from field-collected Helicoverpa armigera populations in Northern China. Southwestern Entomologist 35:399-402.

Gatehouse, A. M. R., N. Ferry, and R. J. M. Raemaekers. 2002. The case of the monarch butterfly: A verdict is returned. Trends in Genetics 18:249-251.

Gatehouse, A. M. R., N. Ferry, M. G. Edwards, and H. A. Bell. 2011. Insect-resistant biotech crops and their impacts on beneficial arthropods. Philosophical Transactions of the Royal Society B-Biological Sciences 366:1438-1452.

Gathmann, A., L. Wirooks, L. A. Hothorn, D. Bartsch, and I. Schuphan. 2006. Impact of $B t$ maize pollen (MON810) on Lepidopteran larvae living on accompanying weeds. Molecular Ecology 15:2677-2685.

Giovannetti, M. 2003. The ecological risks of transgenic plants. Rivista Di BiologiaBiology Forum 96:207-223.

Giovannetti, M., C. Sbrana, and A. Turrini. 2005. The impact of genetically modified crops on soil microbial communities. Rivista Di Biologia-Biology Forum 98:393417.

Gosling, P., A. Hodge, G. Goodlass, and G. D. Bending. 2006. Arbuscular mycorrhizal fungi and organic farming. Agriculture Ecosystems and Environment 113:17-35. 
Gould, F., N. Blair, M. Reid, T. L. Rennie, J. Lopez, and S. Micinski. 2002. Bacillus thuringiensis-toxin resistance management: Stable isotope assessment of alternate host use by Helicoverpa zea. Proceedings of the National Academy of Sciences of the United States of America 99:16581-16586.

Grayston, S. J., D. Vaughan, and D. Jones. 1996. Rhizosphere carbon flow in trees, in comparison with annual plants; the importance of root exudation and its impact on microbial activity and nutrient availability. Applied Soil Ecology 5:29-56.

Griego, V. M., and K. D. Spence. 1978. Inactivation of Bacillus thuringiensis spores by ultraviolet and visible-light. Applied and Environmental Microbiology 35:906910.

Griffiths, B. S., I.E. Geoghegan, and W. M. Robertson. 2000. Testing genetically engineered potato, producing the lectins GNA and Con A, on nontarget soil organisms and processes. Journal of Applied Ecology 37:159-170.

Griffiths, B. S., S. Caul, J. Thompson, A. N. E. Birch, C. Scrimgeour, M. N. Andersen, J. Cortet, A. Messean, C. Sausse, B. Lacroix, and P. H. Krogh. 2005. A comparison of soil microbial community structure, protozoa and nematodes in field plots of conventional and genetically modified maize expressing the Bacillus thuringiensis Cry1Ab toxin. Plant and Soil 275:135-146.

Griffiths, B. S., S. Caul, J. Thompson, A. N. E. Birch, C. Scrimgeour, J. Cortet, A. Foggo, C. A. Hackett, and P. H. Krogh. 2006. Soil microbial and faunal community responses to $B t$ maize and insecticide in two soils. Journal of Environmental Quality 35:734-741.

Griffiths, B. S., S. Caul, J. Thompson, A. N. E. Birch, J. Cortet, M. N. Andersen, and P. H. Krogh. 2007a. Microbial and microfaunal community structure in cropping systems with genetically modified plants. Pedobiologia 51:195-206.

Griffiths, B. S., L. H. Heckmann, S. Caul, J. Thompson, C. Scrimgeour, and P. H. Krogh. 2007b. Varietal effects of eight paired lines of transgenic $B t$ maize and nearisogenic non- $B t$ maize on soil microbial and nematode community structure. Plant Biotechnology Journal 5:60-68.

Griffiths, B. S., S. Caul, J. Thompson, C. A. Hackett, J. Cortet, C. Pernin, and P. H. Krogh. 2008. Soil microbial and faunal responses to herbicide tolerant maize and herbicide in two soils. Plant and Soil 308:93-103.

Groot, A. T. and M. Dicke. 2002. Insect-resistant transgenic plants in a multi-trophic context. Plant Journal 31:387-406.

Guo, J. Y., F. H. Wan, L. Dong, G. L. Lovei, and Z. J. Han. 2008. Tri-trophic interactions between $B t$ cotton, the herbivore Aphis gossypii glover (Homoptera : Aphididae), and the predator Chrysopa pallens (Rambur) (Neuroptera : Chrysopidae).

Environmental Entomology 37:263-270.

Handelsman, J. and J. Tiedje. 2007. The new science of metagenomics. The National Academies Press, Washington, DC. 
Hayat, R., S. Ali, U. Amara, R. Khalid, and I. Ahmed. 2010. Soil beneficial bacteria and their role in plant growth promotion: A review. Annals of Microbiology 60:579598.

Heckmann, L. H., B. S. Griffiths, S. Caul, J. Thompson, M. Pusztai-Carey, W. J. Moar, M. N. Andersen, and P. H. Krogh. 2006. Consequences for Protaphorura armata (Collembola : Onychiuridae) following exposure to genetically modified Bacillus thuringiensis (Bt) maize and non-Bt maize. Environmental Pollution 142:212-216.

Hellmich, R. L., B. D. Siegfried, M. K. Sears, D. E. Stanley-Horn, M. J. Daniels, H. R. Mattila, T. Spencer, K. G. Bidne, and L. C. Lewis. 2001. Monarch larvae sensitivity to Bacillus thuringiensis-purified proteins and pollen. Proceedings of the National Academy of Sciences of the United States of America 98:1192511930.

Hilbeck, A., W. J. Moar, M. Pusztai-Carey, A. Filippini, and F. Bigler. 1999. Preymediated effects of Cry1 Ab toxin and protoxin and Cry2A protoxin on the predator Chrysoperla carnea. Entomologia Experimentalis Et Applicata 91:305316.

Hoess, S., M. Arndt, S. Baurngarte, C. C. Tebbe, H. T. Nguyen, and J. A. Jehle. 2008. Effects of transgenic corn and CrylAb protein on the nematode, Caenorhabditis elegans. Ecotoxicology and Environmental Safety 70:334-340.

Hoess, S., H. T. Nguyen, R. Menzel, S. Pagel-Wieder, R. Miethling-Graf, C. C. Tebbe, J. A. Jehle, and W. Traunspurger. 2011. Assessing the risk posed to free-living soil nematodes by a genetically modified maize expressing the insecticidal Cry3Bb1 protein. Science of the Total Environment 409:2674-2684.

Hofte, H., and H. R. Whiteley. 1989. Insecticidal crystal proteins of Bacillus thuringiensis. Microbiological Reviews 53:242-255.

Honemann, L., C. Zurbrugg, and W. Nentwig. 2008. Effects of Bt-corn decomposition on the composition of the soil meso- and macrofauna. Applied Soil Ecology 40:203209.

Honemann, L. and W. Nentwig. 2009. Are survival and reproduction of Enchytraeus albidus (Annelida: Enchytraeidae) at risk by feeding on Bt-maize litter? European Journal of Soil Biology 45:351-355.

Hopkins, D. W. and E. G. Gregorich. 2003. Detection and decay of the Bt endotoxin in soil from a field trial with genetically modified maize. European Journal of Soil Science 54:793-800.

Hu, H. Y., X. X. Liu, Z. W. Zhao, J. G. Sun, Q. W. Zhang, X. Z. Liu, and Y. Yu. 2009. Effects of repeated cultivation of transgenic $B t$ cotton on functional bacterial populations in rhizosphere soil. World Journal of Microbiology and Biotechnology 25:357-366.

Hu, Y., S. B. Georghiou, A. J. Kelleher, and R. V. Aroian. 2010. Bacillus thuringiensis Cry5B protein is highly efficacious as a single-dose therapy against an intestinal 
roundworm infection in mice. PloS Neglected Tropical Diseases 4.

Huang, F. N., B. R. Leonard, D. R. Cook, D. R. Lee, D. A. Andow, J. L. Baldwin, K. V. Tindall, and X. Y. Wu. 2007. Frequency of alleles conferring resistance to Bacillus thuringiensis maize in Louisiana populations of the southwestern corn borer. Entomologia Experimentalis Et Applicata 122:53-58.

Hutchison, W. D., E. C. Burkness, P. D. Mitchell, R. D. Moon, T. W. Leslie, S. J. Fleischer, M. Abrahamson, K. L. Hamilton, K. L. Steffey, M. E. Gray, R. L. Hellmich, L. V. Kaster, T. E. Hunt, R. J. Wright, K. Pecinovsky, T. L. Rabaey, B. R. Flood, and E. S. Raun. 2010. Areawide suppression of European corn borer with $B t$ maize reaps savings to non-Bt maize growers. Science 330:222-225.

Icoz, I., D. Saxena, D. A. Andow, C. Zwahlen, and G. Stotzky. 2008. Microbial populations and enzyme activities in soil in situ under transgenic corn expressing Cry proteins from Bacillus thuringiensis. Journal of Environmental Quality 37:647-662.

Icoz, I. and G. Stotzky. 2008a. Cry3Bb1 protein from Bacillus thuringiensis in root exudates and biomass of transgenic corn does not persist in soil. Transgenic Research 17:609-620.

Icoz, I. and G. Stotzky. 2008b. Fate and effects of insect-resistant Bt crops in soil ecosystems. Soil Biology and Biochemistry 40:559-586.

Ito, A., Y. Sasaguri, S. Kitada, Y. Kusaka, K. Kuwano, K. Masutomi, E. Mizuki, T. Akao, and M. Ohba. 2004. A Bacillus thuringiensis crystal protein with selective cytocidal action to human cells. Journal of Biological Chemistry 279:2128221286.

James, C. 2010. Global Status of Commercialized Biotech/GM Crops: 2010. International Service for the Acquisition of Agri-Biotech Applications, Ithaca, NY. http://isaaa.org/resources/publications/briefs/42/executivesummary/default.asp

Jensen, P. D., G. P. Dively, C. M. Swan, and W. O. Lamp. 2010. Exposure and nontarget effects of transgenic Bt corn debris in streams. Environmental Entomology 39:707-714.

Jesse, L. C. H. and J. J. Obrycki. 2000. Field deposition of Bt transgenic corn pollen: Lethal effects on the monarch butterfly. Oecologia 125:241-248.

Jung, H. G. and C. C. Sheaffer. 2004. Lignin concentration of whole plants and stems of Bt corn hybrids. Journal of Animal Science 82:250-250.

Khan, M. Q., M. W. Abbasi, M. J. Zaki, and S. A. Khan. 2010. Evaluation of Bacillus thuringiensis isolates against root-knot nematodes following seed application in okra and mungbean. Pakistan Journal of Botany 42:2903-2910.

Knox, O. G. G., D. B. Nehl, T. Mor, G. N. Roberts, and V. Gupta. 2008. Genetically modified cotton has no effect on arbuscular mycorrhizal colonisation of roots. Field Crops Research 109:57-60.

Koskella, J. and G. Stotzky. 2002. Larvicidal toxins from Bacillus thuringiensis subspp. 
kurstaki, morrisoni (strain tenebrionis), and israelensis have no microbicidal or microbiostatic activity against selected bacteria, fungi, and algae in vitro. Canadian Journal of Microbiology 48:262-267.

Kotze, A. C., J. O'Grady, J. M. Gough, R. Pearson, N. H. Bagnall, D. H. Kemp, and R. J. Akhurst. 2005. Toxicity of Bacillus thuringiensis to parasitic and free-living lifestages of nematode parasites of livestock. International Journal for Parasitology 35:1013-1022.

Kowalchuk, G. A., D. S. Buma, W. de Boer, P. G. L. Klinkhamer, and J. A. van Veen. 2002. Effects of above-ground plant species composition and diversity on the diversity of soil-borne microorganisms. Antonie van Leeuwenhoek 81:509-520.

Kowalchuk, G. A., M. Bruinsma, and J. A. van Veen. 2003. Assessing responses of soil microorganisms to GM plants. Trends in Ecology and Evolution 18:403-410.

Kravchenko, A. N., X. M. Hao, and G. P. Robertson. 2009. Seven years of continuously planted $B t$ corn did not affect mineralizable and total soil $\mathrm{C}$ and total $\mathrm{N}$ in surface soil. Plant and Soil 318:269-274.

Krogh, P. H., B. Griffiths, D. Demsar, M. Bohanec, M. Debeljak, M. N. Andersen, C. Sausse, A. N. E. Birch, S. Caul, M. Holmstrup, L. H. Heckmann, and J. Cortet. 2007. Responses by earthworms to reduced tillage in herbicide tolerant maize and Bt maize cropping systems. Pedobiologia 51:219-227.

Lamarche, J., and R. C. Hamelin. 2007. No evidence of an impact on the rhizosphere diazotroph community by the expression of Bacillus thuringiensis Cry1Ab toxin by $B t$ white spruce. Applied and Environmental Microbiology 73:6577-6583.

Lang, A., C. Ludy, and E. Vojtech. 2004. Dispersion and deposition of Bt maize pollen in field margins. Zeitschrift Fur Pflanzenkrankheiten Und Pflanzenschutz-Journal of Plant Diseases and Protection 111:417-428.

Lang, A., K. Abdel-Kader, M. Arndt, J. Bauchhenss, R. Beck, U. Benker, A. Hermann, D. Mautz, M. Zellner, and G. Pommer. 2006. Monitoring the environmental impact of $B t$ maize: A research project of the Bavarian State Ministry for Health, Environment and Consumer Protection, and the Bavarian State Research Center for Agriculture. Mitteilungen aus der Biologischen Bundesanstalt fuer Land- und Forstwirtschaft Berlin-Dahlem 403:136-139.

Lang, A. and E. Vojtech. 2006. The effects of pollen consumption of transgenic Bt maize on the common swallowtail, Papilio machaon L. (Lepidoptera, Papilionidae). Basic and Applied Ecology 7:296-306.

Lang, A. and M. Otto. 2010. A synthesis of laboratory and field studies on the effects of transgenic Bacillus thuringiensis (Bt) maize on nontarget Lepidoptera. Entomologia Experimentalis Et Applicata 135:121-134.

Lee, L., D. Saxena, and G. Stotzky. 2003. Activity of free and clay-bound insecticidal proteins from Bacillus thuringiensis subsp israelensis against the mosquito Culex pipiens. Applied and Environmental Microbiology 69:4111-4115. 
Lehman, R. M., S. L. Osborne, and K. A. Rosentrater. 2008. No differences in decomposition rates observed between Bacillus thuringiensis and non-Bacillus thuringiensis corn residue incubated in the field. Agronomy Journal 100:163-168.

Lekberg, Y., R. T. Koide, and S. J. Twomlow. 2008. Effect of agricultural management practices on arbuscular mycorrhizal fungal abundance in low-input cropping systems of southern Africa: A case study from Zimbabwe. Biology and Fertility of Soils 44:917-923.

Li, X., A. Tan, M. Voegtline, S. Bekele, J. Wei, and R. V. Aroian. 2007a. Nematicidal Bt crystal proteins targeting plant endoparasitic nematodes. Phytopathology 97:S144-S144.

Li, X. Q., J. Z. Wei, A. Tan, and R. V. Aroian. 2007b. Resistance to root-knot nematode in tomato roots expressing a nematicidal Bacillus thuringiensis crystal protein. Plant Biotechnology Journal 5:455-464.

Li, X. Q., A. Tan, M. Voegtline, S. Bekele, C. S. Chen, and R. V. Aroian. 2008. Expression of Cry5B protein from Bacillus thuringiensis in plant roots confers resistance to root-knot nematode. Biological Control 47:97-102.

Li, X. G., B. A. Liu, S. Heia, D. D. Liu, Z. M. Han, K. X. Zhou, J. J. Cui, J. Y. Luo, and Y. P. Zheng. 2009. The effect of root exudates from two transgenic insectresistant cotton lines on the growth of Fusarium oxysporum. Transgenic Research 18:757-767.

Li, X. G., B. A. Liu, J. J. Cui, D. D. Liu, S. A. Ding, B. Gilna, J. Y. Luo, Z. X. Fang, W. Cao, and Z. M. Han. 2011. No evidence of persistent effects of continuously planted transgenic insect-resistant cotton on soil microorganisms. Plant and Soil 339:247-257.

Liu, B., Q. Zeng, F. M. Yan, H. G. Xu, and C. R. Xu. 2005. Effects of transgenic plants on soil microorganisms. Plant and Soil 271:1-13.

Liu, W. and L. F. Du. 2008. Interactions between $B t$ transgenic crops and arbuscular mycorrhizal fungi: A new urgent issue of soil ecology in agroecosystems. Acta Agriculturae Scandinavica Section B-Soil and Plant Science 58:187-192.

Liu, W., H. H. Lu, W. Wu, Q. K. Wei, Y. X. Chen, and J. E. Thies. 2008. Transgenic Bt rice does not affect enzyme activities and microbial composition in the rhizosphere during crop development. Soil Biology and Biochemistry 40:475-486.

Liu, B., J. J. Cui, J. Meng, W. J. Hu, J. Y. Luo, and Y. P. Zheng. 2009a. Effects of transgenic Bt plus CpTI cotton on the growth and reproduction of earthworm Eisenia foetida. Frontiers in Bioscience 14:4008-4014.

Liu, B., L. Wang, Q. Zeng, J. Meng, W. J. Hu, X. G. Li, K. X. Zhou, K. Xue, D. D. Liu, and Y. P. Zheng. 2009b. Assessing effects of transgenic Cry1Ac cotton on the earthworm Eisenia fetida. Soil Biology and Biochemistry 41:1841-1846.

Liu, W. K. 2010. Do genetically modified plants impact arbuscular mycorrhizal fungi? Ecotoxicology 19:229-238. 
Losey, J. E., L. S. Rayor, and M. E. Carter. 1999. Transgenic pollen harms monarch larvae. Nature 399:214-214.

Lottman, J., H. Heier, J. de Vries, A. Mahn, K. During, W. Wackernagel, K. Smalla, and G. Berg. 2000. Establishment of introduced antagonistic bacteria in the rhizosphere of transgenic potatoes and their effect on the bacterial community. FEMS Microbiology Ecology 33:41-49.

Lovei, G. L., D. A. Andow, and S. Arpaia. 2009. Transgenic insecticidal crops and natural enemies: A detailed review of laboratory studies. Environmental Entomology 38:293-306.

Lu, H. H., W. X. Wu, Y. X. Chen, H. L. Wang, M. Devare, and J. E. Thies. 2010a. Soil microbial community responses to $B t$ transgenic rice residue decomposition in a paddy field. Journal of Soils and Sediments 10:1598-1605.

Lu, H. H., W. X. Wu, Y. X. Chen, X. J. Zhang, M. Devare, and J. E. Thies. 2010b. Decomposition of $B t$ transgenic rice residues and response of soil microbial community in rapeseed-rice cropping system. Plant and Soil 336:279-290.

Lundgren, J. G., A. J. Gassmann, J. Bernal, J. J. Duan, and J. Ruberson. 2009. Ecological compatibility of GM crops and biological control. Crop Protection 28:1017-1030.

Lupwayi, N. Z., K. G. Hanson, K. N. Harker, G. W. Clayton, R. E. Blackshaw, J. T. O'Donovan, E. N. Johnson, Y. Gan, R. B. Irvine, and M. A. Monreal. 2007. Soil microbial biomass, functional diversity and enzyme activity in glyphosateresistant wheat-canola rotations under low-disturbance direct seeding and conventional tillage. Soil Biology and Biochemistry 39:1418-1427.

Manachini, B., and G. C. Lozzia. 2002. First investigations into the effects of $B t$ corn crop on Nematofauna. Bollettino di Zoologia Agraria e di Bachicoltura 34:85-96.

Manachini, B. and G. C. Lozzia. 2003. Biodiversity and structure on Nematofauna in $B t$ corn Pp. 32. Biodiversity Implications of Genetically Modified Plants, Ascona, Switzerland.

Manachini, B., M. C. Fiore, S. Landi, and S. Arpaia. 2003. Nematode species assemblage in Bt-expressing transgenic eggplants and their isogenic control. Pp. 31.

Biodiversity Implications of Genetically Modified Plants, Ascona, Switzerland.

Manachini, B., S. Landi, M. C. Fiore, M. Festa, and S. Arpaia. 2004. First investigations on the effects of Bt-transgenic Brassica nupus L. on the trophic structure of the nematofauna. Pp. 103-108. IOBC/WPRS Bulletin.

Marroquin, L. D., D. Elyassnia, J. S. Griffitts, J. S. Feitelson, and R. V. Aroian. 2000. Bacillus thuringiensis $(\mathrm{Bt})$ toxin susceptibility and isolation of resistance mutants in the nematode Caenorhabditis elegans. Genetics 155:1693-1699.

Marvier, M., C. McCreedy, J. Regetz, and P. Kareiva. 2007. A meta-analysis of effects of Bt cotton and maize on nontarget invertebrates. Science 316:1475-1477.

Meadows, J., S. S. Gill, and L. W. Bone. 1989a. Factors influencing lethality of Bacillus thuringiensis-kurstaki toxin for eggs and larvae of Trichostronglus 
columbriformis (Nematoda). Journal of Parasitology 75:191-194.

Meadows, J. R., S. S. Gill, and L. W. Bone. 1989b. Lethality of Bacillus thuringiensis morrisoni for eggs of Trichostronglus colubriformis (Nematoda). Invertebrate Reproduction and Development 15:159-161.

Meadows, J., S. S. Gill, and L. W. Bone. 1990. Bacillus thuringiensis strains affect population-growth of the free-living nematode Turbatrix aceti. Invertebrate Reproduction and Development 17:73-76.

Menninger, H. L. and M. A. Palmer. 2007. Herbs and grasses as an allochthonous resource in open-canopy headwater streams. Freshwater Biology 52:1689-1699.

Mercer, K. L. and J. D. Wainwright. 2008. Gene flow from transgenic maize to landraces in Mexico: An analysis. Agriculture Ecosystems and Environment 123:109-115.

Miethling-Graff, R., S. Dockhorn, and C. C. Tebbe. 2010. Release of the recombinant Cry3Bb1 protein of $\mathrm{Bt}$ maize MON88017 into field soil and detection of effects on the diversity of rhizosphere bacteria. European Journal of Soil Biology 46:4148.

Monsanto. 2011. Research and Development Pipeline, St. Louis, MO. http://www.monsanto.com/products/Pages/research-developmentpipeline.aspx\#/Overview

Moore, A. A. and M. A. Palmer. 2005. Invertebrate biodiversity in agricultural and urban headwater streams: Implications for conservation and management. Ecological Applications 15:1169-1177.

Morgan, J. A. W., G. D. Bending, and P. J. White. 2005. Biological costs and benefits to plant-microbe interactions in the rhizosphere. Journal of Experimental Botany 56:1729-1739.

Motavalli, P. P., R. J. Kremer, M. Fang, and N. E. Means. 2004. Impact of genetically modified crops and their management on soil microbially mediated plant nutrient transformations. Journal of Environmental Quality 33:816-824.

Mungai, N. W., P. P. Motavalli, K. A. Nelson, and R. J. Kremer. 2005. Differences in yields, residue composition and $\mathrm{N}$ mineralization dynamics of $B t$ - and non- $B t$ maize. Nutrient Cycling in Agroecosystems 73:101-109.

Naef, A., T. Zesiger, and G. Defago. 2006. Impact of transgenic Bt maize residues on the mycotoxigenic plant pathogen Fusarium graminearum and the biocontrol agent Trichoderma atroviride. Journal of Environmental Quality 35:1001-1009.

Nagamatsu, Y., S. Okamura, H. Saitou, T. Akao, and E. Mizuki. 2010. Three Cry toxins in two types from Bacillus thuringiensis strain M019 preferentially kill human hepatocyte cancer and uterus cervix cancer cells. Bioscience Biotechnology and Biochemistry 74:494-498.

O'Callaghan, M., T. R. Glare, E. P. J. Burgess, and L. A. Malone. 2005. Effects of plants genetically modified for insect resistance on nontarget organisms. Annual Review of Entomology 50:271-292. 
Ohba, M., E. Mizuki, and A. Uemori. 2009. Parasporin, a new anticancer protein group from Bacillus thuringiensis. Anticancer Research 29:427-433.

Oliveira, A. P., M. E. Pampulha, and J. P. Bennett. 2008. A two-year field study with transgenic Bacillus thuringiensis maize: Effects on soil microorganisms. Science of the Total Environment 405:351-357.

Oliveira, A. R., T. R. Castro, D. M. F. Capalbo, and I. Delalibera. 2007. Toxicological evaluation of genetically modified cotton (Bollgard $®)$ and Dipel®WP on the nontarget soil mite Scheloribates praeincisus (Acari : Oribatida). Experimental and Applied Acarology 41:191-201.

Ortiz-Garcia, S., E. Ezcurra, B. Schoel, F. Acevedo, J. Sobernon, and A. A. Snow. 2005. Absence of detectable transgenes in local landraces of maize in Oaxaca, Mexico (2003-2004). Proceedings of the National Academy of Sciences of the United States of America 102:12338-12343.

Pineyro-Nelson, A., J. Van Heerwaarden, H. R. Perales, J. A. Serratos-Hernandez, A. Rangel, M. B. Hufford, P. Gepts, A. Garay-Arroyo, R. Rivera-Bustamante, and E. R. Alvarez-Buylla. 2009. Transgenes in Mexican maize: Molecular evidence and methodological considerations for GMO detection in landrace populations. Molecular Ecology 18:750-761.

Poerschmann, J., A. Gathmann, J. Augustin, U. Langer, and T. Gorecki. 2005. Molecular composition of leaves and stems of genetically modified $B t$ and near-isogenic non-Bt maize - Characterization of lignin patterns. Journal of Environmental Quality 34:1508-1518.

Pont, B. and W. Nentwig. 2005. Quantification of Bt-protein digestion and excretion by the primary decomposer Porcellio scaber, fed with two Bt-corn varieties. Biocontrol Science and Technology 15:341-352.

Poornima, K., P. Selvanayagam, and R. Shenbagarathai. 2010. Identification of native Bacillus thuringiensis strain from South India having specific cytocidal activity against cancer cells. Journal of Applied Microbiology 109:348-354.

Prasifka, P. L., R. L. Hellmich, J. R. Prasifka, and L. C. Lewis. 2007. Effects of Cry1Abexpressing corn anthers on the movement of monarch butterfly larvae. Environmental Entomology 36:228-233.

Priestley, A. L. and M. Brownbridge. 2009. Field trials to evaluate effects of Bttransgenic silage corn expressing the Cry1 Ab insecticidal toxin on nontarget soil arthropods in northern New England, USA. Transgenic Research 18:425-443.

Prihoda, K. R. and J. R. Coats. 2008. Aquatic fate and effects of Bacillus thuringiensis Cry3Bb1 protein: Toward risk assessment. Environmental Toxicology and Chemistry 27:793-798.

Quist, D. and I. H. Chapela. 2001. Transgenic DNA introgressed into traditional maize landraces in Oaxaca, Mexico. Nature 414:541-543.

Reichman, J. R., L. S. Watrud, E. H. Lee, C. A. Burdick, M. A. Bollman, M. J. Storm, G. 
A. King, and C. Mallory-Smith. 2006. Establishment of transgenic herbicideresistant creeping bentgrass (Agrostis stolonifera L.) in nonagronomic habitats. Molecular Ecology 15:4243-4255.

Rillig, M. C. 2004. Arbuscular mycorrhizae and terrestrial ecosystem processes. Ecology Letters 7:740-754.

Romeis, J., M. Meissle, and F. Bigler. 2006. Transgenic crops expressing Bacillus thuringiensis toxins and biological control. Nature Biotechnology 24:63-71.

Rosi-Marshall, E. J., J. L. Tank, T. V. Royer, M. R. Whiles, M. Evans-White, C. Chambers, N. A. Griffiths, J. Pokelsek, and M. L. Stephen. 2007. Toxins in transgenic crop byproducts may affect headwater stream ecosystems. Proceedings of the National Academy of Sciences of the United States of America 104:1620416208.

Rui, Y. K., G. X. Yi, J. Zhao, B. M. Wang, Z. H. Li, Z. X. Zhai, Z. P. He, and Q. X. Li. 2005. Changes of $B t$ toxin in the rhizosphere of transgenic $B t$ cotton and its influence on soil functional bacteria. World Journal of Microbiology and Biotechnology 21:1279-1284.

Sanchis, V. 2011. From microbial sprays to insect-resistant transgenic plants: History of the biospesticide Bacillus thuringiensis. A review. Agronomy for Sustainable Development 31:217-231.

Saxena, D., S. Flores, and G. Stotzky. 1999. Insecticidal toxin in root exudates from Bt corn. Nature 402:480.

Saxena, D. and G. Stotzky. 2000. Insecticidal toxin from Bacillus thuringiensis is released from roots of transgenic Bt corn in vitro and in situ. FEMS Microbiology Ecology 33:35-39.

Saxena, D. and G. Stotzky. 2001a. Bacillus thuringiensis (Bt) toxin released from root exudates and biomass of $B t$ corn has no apparent effect on earthworms, nematodes, protozoa, bacteria, and fungi in soil. Soil Biology and Biochemistry 33:1225-1230.

Saxena, D. and G. Stotzky. 2001b. Bt corn has a higher lignin content than non-Bt corn. American Journal of Botany 88:1704-1706.

Saxena, D. and G. Stotzky. 2001c. Bt toxin uptake from soil by plants. Nature Biotechnology 19:199-199.

Saxena, D. and G. Stotzky. 2002. Bt toxin is not taken up from soil or hydroponic culture by corn, carrot, radish, or turnip. Plant and Soil 239:165-172.

Saxena, D., S. Flores, and G. Stotzky. 2002a. Bt toxin is released in root exudates from 12 transgenic corn hybrids representing three transformation events. Soil Biology and Biochemistry 34:133-137.

Saxena, D., S. Flores, and G. Stotzky. 2002b. Vertical movement in soil of insecticidal Cry1Ab protein from Bacillus thuringiensis. Soil Biology and Biochemistry 34:111-120. 
Saxena, D. and G. Stotzky. 2003. Fate and effects in soil of insecticidal toxins from Bacillus thuringiensis in transgenic plants. Pp. 7-83. Collection of Biosafety Reviews. International Centre for Genetic Engineering and Biotechnology, Trieste.

Saxena, D., C. N. Stewart, I. Altosaar, Q. Y. Shu, and G. Stotzky. 2004. Larvicidal Cry proteins from Bacillus thuringiensis are released in root exudates of transgenic $B$. thuringiensis corn, potato, and rice but not of $B$. thuringiensis canola, cotton, and tobacco. Plant Physiology and Biochemistry 42:383-387.

Saxena, D., S. Pushalkar, and G. Stotzky. 2010. Fate and effects in soil of Cry proteins from Bacillus thuringiensis in sprays and transgenic plants: Influence of the physicochemical and biological characteristics of soil. In Entomopathogenic Bacterial Toxins as Biological Control Agents, The Open Toxinology Journal. eds. E. Ben-Dov and A. Zaritsky, 133-153. Oak Park, IL: Bentham Science Publishers Ltd.

Schnepf, E., N. Crickmore, J. Van Rie, D. Lereclus, J. Baum, J. Feitelson, D. R. Zeigler, and D. H. Dean. 1998. Bacillus thuringiensis and its pesticidal crystal proteins. Microbiology and Molecular Biology Reviews 62:775-806.

Schrader, S., T. Munchenberg, S. Baumgarte, and C. C. Tebbe. 2008. Earthworms of different functional groups affect the fate of the $B t$-toxin Cry1Ab from transgenic maize in soil. European Journal of Soil Biology 44:283-289.

Sears, M. K., R. L. Hellmich, D. E. Stanley-Horn, K. S. Oberhauser, J. M. Pleasants, H. R. Mattila, B. D. Siegfried, and G. P. Dively. 2001. Impact of Bt corn pollen on monarch butterfly populations: A risk assessment. Proceedings of the National Academy of Sciences of the United States of America 98:11937-11942.

Sears, M. K. 2004. Impact of Bacillus thuringiensis corn pollen on monarch butterfly populations: A risk assessment. Agricultural Biotechnology: Challenges and Prospects 866:125-137.

Sessitsch, A., J. G. Howieson, X. Perret, H. Antoun, and E. Martinez-Romero. 2002. Advances in Rhizobium research. Critical Reviews in Plant Sciences 21:323-378.

Shelton, A. M. and M. K. Sears. 2001. The monarch butterfly controversy: Scientific interpretations of a phenomenon. The Plant Journal 27:483-488.

Shen, R. F., H. Cai, and W. H. Gong. 2006. Transgenic Bt cotton has no apparent effect on enzymatic activities or functional diversity of microbial communities in rhizosphere soil. Plant and Soil 285:149-159.

Sheng, M., M. Tang, H. Chen, B. W. Yang, F. F. Zhang, and Y. H. Huang. 2008. Influence of arbuscular mycorrhizae on photosynthesis and water status of maize plants under salt stress. Mycorrhiza 18:287-296.

Sheveleva, E. V., S. Marquez, W. Chmara, A. Zegeer, R. G. Jensen, and H. J. Bohnert. 1998. Sorbitol-6-phosphate dehydrogenase expression in transgenic tobacco High amounts of sorbitol lead to necrotic lesions. Plant Physiology 117:831-839. 
Shu, Q. Y., H. R. Cui, G. Y. Ye, D. X. Wu, Y. W. Xia, M. W. Gao, and I. Altosaar. 2002. Agronomic and morphological characterization of Agrobacterium-transformed $\mathrm{Bt}$ rice plants. Euphytica 127:345-352.

Siciliano, S. D. and J. J. Germida. 1999. Taxonomic diversity of bacteria associated with the roots of field-grown transgenic Brassica napus cv. Quest, compared to the non-transgenic B. napus cv. Excel and B. rapa cv. Parkland. FEMS Microbiology Ecology 29:263-272.

Sims, S. R. 1997. Host activity spectrum of the CryIIA Bacillus thuringiensis subsp. kurstaki protein: Effect on Lepidoptera, Diptera, and nontarget arthropods. Southwestern Entomologist 22:395-404.

Sims, S. R. and J. W. Martin. 1997. Effect of the Bacillus thuringiensis insecticidal proteins CryIA(b), CryIA(c), CryIIA, and CryIIIA on Folsomia candida and Xenylla grisea (Insecta: Collembola). Pedobiologia 41:412-416.

Smith, S. E. and V. Gianinazzi-Pearson. 1988. Physiological interactions between symbionts in vesicular-arbuscular mycorrhizal plants. Annual Review of Plant Physiology and Plant Molecular Biology 39:221-244.

Smith, S. E. and D. J. Read. 2008. Mycorrhizal Symbiosis. Academic Press, London. Snow, A. A. 2002. Transgenic crops - why gene flow matters. Nature Biotechnology 20:542-542.

Stanley-Horn, D. E., G. P. Dively, R. L. Hellmich, H. R. Mattila, M. K. Sears, R. Rose, L. C. H. Jesse, J. E. Losey, J. J. Obrycki, and L. Lewis. 2001. Assessing the impact of Cry1 Ab-expressing corn pollen on monarch butterfly larvae in field studies. Proceedings of the National Academy of Sciences of the United States of America 98:11931-11936.

Stotzky, G. 2000. Persistence and biological activity in soil of insecticidal proteins from Bacillus thuringiensis and of bacterial DNA bound on clays and humic acids. Journal of Environmental Quality 29:691-705.

Stotzky, G. 2002. Release, persistence, and biological activity in soil of insecticidal proteins from Bacillus thuringiensis. In Genetically Engineered Organisms: Assessing Environmental and Human Health Effects, eds. D. K. Letourneau and B. E. Burrows, 187-222. Boca Raton, FL: CRC Press.

Stotzky, G. 2004. Persistence and biological activity in soil of the insecticidal proteins from Bacillus thuringiensis, especially from transgenic plants. Plant and Soil 266:77-89.

Stotzky, G. and D. Saxena. 2009. Is molecular "pharming" a potential hazard to the environment? In Environmental Impact Assessments, eds., G. T. Halley and Y. T. Fridian, 77-86. Hauppauge, NY: Nova Science Publishers, Inc.

Sun, C. X., L. J. Chen, Z. J. Wu, L. K. Zhou, and H. Shimizu. 2007. Soil persistence of Bacillus thuringiensis $(B t)$ toxin from transgenic $B t$ cotton tissues and its effect on soil enzyme activities. Biology and Fertility of Soils 43:617-620. 
Swan, C. M., P. D. Jensen, G. P. Dively, and W. O. Lamp. 2009. Processing of transgenic crop residues in stream ecosystems. Journal of Applied Ecology 46:1304-1313.

Tan, F. X., J. W. Wang, Y. J. Feng, G. L. Chi, H. L. Kong, H. F. Qiu, and S. L. Wei. 2010. Bt corn plants and their straw have no apparent impact on soil microbial communities. Plant and Soil 329:349-364.

Tank, J. L., E. J. Rosi-Marshall, T. V. Royer, M. R. Whiles, N. A. Griffiths, T. C. Frauendorf, and D. J. Treering. 2010. Occurrence of maize detritus and a transgenic insecticidal protein (Cry1 Ab) within the stream network of an agricultural landscape. Proceedings of the National Academy of Sciences of the United States of America 107:17645-17650.

Tapp, H., L. Calamai, and G. Stotzky. 1994. Adsorption and binding of the insecticidal proteins from Bacillus thuringiensis subsp. kurstaki and subsp. tenebrionis on clay minerals. Soil Biology and Biochemistry 26:663-679.

Tapp, H. and G. Stotzky. 1995. Insecticidal activity of the toxins from Bacillus thuringiensis subspecies kurstaki and tenebrionis adsorbed and bound on pure and soil clays. Applied and Environmental Microbiology 61:1786-1790.

Tapp, H. and G. Stotzky. 1998. Persistence of the insecticidal toxin from Bacillus thuringiensis subsp. kurstaki in soil. Soil Biology and Biochemistry 30:471-476.

Tarkalson, D. D., S. D. Kachman, J. M. N. Knops, J. E. Thies, and C. S. Wortmann. 2008. Decomposition of $B t$ and non-Bt corn hybrid residues in the field. Nutrient Cycling in Agroecosystems 80:211-222.

Tharakan, B. D., G. Devaki, H. B. Nair, and J. Padikkala. 2009. Cytotoxic effects of crystal proteins from Bacillus thuringiensis against breast cancer cells. Cancer Research 69:226S-226S.

Theissen, B. and D. J. Russell. 2009. The relevance of Collembola in monitoring soilecological effects of GMOs. Gefahrstoffe Reinhaltung Der Luft 69:391-394.

Then, C. 2010. Risk assessment of toxins derived from Bacillus thuringiensis-synergism, efficacy, and selectivity. Environmental Science and Pollution Research 17:791797.

Thies, J. E. and M. H. Devare. 2007. An ecological assessment of transgenic crops. Journal of Development Studies 43:97-129.

Tschenn, J., J. E. Losey, L. H. Jesse, J. J. Obrycki, and R. Hufbauer. 2001. Effects of corn plants and corn pollen on monarch butterfly (Lepidoptera : Danaidae) oviposition behavior. Environmental Entomology 30:495-500.

Turrini, A., C. Sbrana, M. P. Nuti, B. M. Pietrangeli, and M. Giovanetti. 2004. Development of a model system to assess the impact of genetically modified corn and aubergine plants on arbuscular mycorrhizal fungi. Plant and Soil 266:69-75.

USDA, U. S. Department of Agriculture, National Agricultural Statistics Service (NASS), 2010. Adoption of Genetically Engineered Crops in the U.S.: Corn Varieties. Extent of Adoption, Table 1. 
http://www.ers.usda.gov/Data/BiotechCrops/ExtentofAdoptionTable1.htm

Vance, C. P., C. Uhde-Stone, and D. L. Allan. 2003. Phosphorus acquisition and use: Critical adaptations by plants for securing a non-renewable resource. New Phytologist 157:423-447.

Vercesi, M. L., P. H. Krogh, and M. Holmstrup. 2006. Can Bacillus thuringiensis (Bt) corn residues and $B t$-corn plants affect life-history traits in the earthworm Aporrectodea caliginosa? Applied Soil Ecology 32:180-187.

Viktorov, A. G. 2011. Transfer of $B t$ corn byproducts from terrestrial to stream ecosystems. Russian Journal of Plant Physiology 58:543-548.

Wandeler, H., J. Bahylova, and W. Nentwig. 2002. Consumption of two Bt and six non$B t$ corn varieties by the woodlouse Porcellio scaber. Basic and Applied Ecology 3:357-365.

Wang, B. and Y. L. Qiu. 2006. Phylogenetic distribution and evolution of mycorrhizas in land plants. Mycorrhiza 16:299-363.

Wang, W. X., B. Vinocur, and A. Altman. 2003. Plant responses to drought, salinity and extreme temperatures: Towards genetic engineering for stress tolerance. Planta 218:1-14.

Watrud, L. S., E. H. Lee, A. Fairbrother, C. Burdick, J. R. Reichman, M. Bollman, M. Storm, G. King, and P. K. Van de Water. 2004. Evidence for landscape-level, pollen-mediated gene flow from genetically modified creeping bentgrass with CP4 EPSPS as a marker. Proceedings of the National Academy of Sciences of the United States of America 101:14533-14538.

Watrud, L. S., G. King, J. P. Londo, R. Colasanti, B. M. Smith, R. S. Waschmann, and E. H. Lee. 2011. Changes in constructed Brassica communities treated with glyphosate drift. Ecological Applications 21:525-538.

West, A. W. 1984. Fate of the insecticidal, Proteinaceous parasporal crystal of Bacillus thuringiensis in soil. Soil Biology and Biochemistry 16:357-360.

West, A. W., H. D. Burges, T. J. Dixon, and C. H. Wyborn. 1985. Survival of Bacillus thuringiensis and Bacillus cereus spore inocula in soil - Effects of $\mathrm{pH}$, moisture, nutrient availability and indigenous microorganisms. Soil Biology and Biochemistry 17:657-665.

Wolfenbarger, L. L., S. E. Naranjo, J. G. Lundgren, R. J. Bitzer, and L. S. Watrud. 2008. $B t$ crop effects on functional guilds of nontarget arthropods: A meta-analysis. PloS One 3:e2118.

Wolt, J. D. and R. K. D. Peterson. 2010. Prospective formulation of environmental risk assessments: Probabilistic screening for Cry1A(b) maize risk to aquatic insects. Ecotoxicology and Environmental Safety 73:1182-1188.

Wong, R. S. Y., S. M. Mohamed, V. D. Nadarajah, and I. A. T. Tengku. 2010. Characterisation of the binding properties of Bacillus thuringiensis 18 toxin on leukaemic cells. Journal of Experimental and Clinical Cancer Research 29. 
Wozniak, C. 2011. U.S. Environmental Protection Agency Biopesticides and Pollution Prevention Division, Washington, DC, USA. Personal communication, October 3, 2011.

Wraight, C. L., A. R. Zangerl, M. J. Carroll, and M. R. Berenbaum. 2000. Absence of toxicity of Bacillus thuringiensis pollen to black swallowtails under field conditions. Proceedings of the National Academy of Sciences of the United States of America 97:7700-7703.

Wu, W. X., Q. F. Ye, and H. Min. 2004a. Effect of straws from Bt-transgenic rice on selected biological activities in water-flooded soil. European Journal of Soil Biology 40:15-22.

Wu, W. X., Q. F. Ye, H. Min, X. J. Duan, and W. M. Jin. 2004b. Bt-transgenic rice straw affects the culturable microbiota and dehydrogenase and phosphatase activities in a flooded paddy soil. Soil Biology and Biochemistry 36:289-295.

Wu, W. X., H. H. Lu, W. Liu, M. Devare, J. E. Thies, and Y. X. Chen. 2009. Decomposition of Bacillus thuringiensis (Bt) transgenic rice residues (straw and roots) in paddy fields. Journal of Soils and Sediments 9:457-467.

Xu, B. L., Y. X. Wang, X. X. Liu, F. Yuan, N. Su, Y. Chen, Y. J. Wu, and Q. W. Zhang. 2006. Effects of Cry1Ac and secondary metabolites in Bt transgenic cottonseed on Lycoriella pleuroti Yang et Zhang (Diptera : Sciaridae). Environmental Entomology 35:807-810.

Xue, K., H. F. Luo, H. Y. Qi, and H. X. Zhang. 2005. Changes in soil microbial community structure associated with two types of genetically engineered plants analyzing by PLFA. Journal of Environmental Sciences-China 17:130-134.

Xue, K., R. C. Serohijos, M. Devare, and J. E. Thies. 2011. Decomposition rates and residue-colonizing microbial communities of Bacillus thuringiensis insecticidal protein Cry3Bb-expressing $(B t)$ and non-Bt corn hybrids in the field. Applied and Environmental Microbiology 77:839-846.

Yamashita, S., H. Katayama, H. Saitoh, T. Akao, Y. S. Park, E. Mizuki, M. Ohba, and A. Ito. 2005. Typical three-domain cry proteins of Bacillus thuringiensis strain A1462 exhibit cytocidal activity on limited human cancer cells. Journal of Biochemistry 138:663-672.

Yu, L., R. E. Berry, and B. A. Croft. 1997. Effects of Bacillus thuringiensis toxins in transgenic cotton and potato on Folsomia candida (Collembola: Isotomidae) and Oppia nitens (Acari: Orbatidae). Journal of Economic Entomology 90:113-118.

Zangerl, A. R., D. McKenna, C. L. Wraight, M. Carroll, P. Ficarello, R. Warner, and M. R. Berenbaum. 2001. Effects of exposure to event 176 Bacillus thuringiensis corn pollen on monarch and black swallowtail caterpillars under field conditions. Proceedings of the National Academy of Sciences of the United States of America 98:11908-11912.

Zeilinger, A. R., D. A. Andow, C. Zwahlen, and G. Stotzky. 2010. Earthworm 
populations in a northern US Cornbelt soil are not affected by long-term cultivation of $B t$ maize expressing Cry1 Ab and Cry3Bb1 proteins. Soil Biology and Biochemistry 42:1284-1292.

Zwahlen, C., A. Hilbeck, P. Gugerli, and W. Nentwig. 2003a. Degradation of the Cry1Ab protein within transgenic Bacillus thuringiensis corn tissue in the field. Molecular Ecology 12:765-775.

Zwahlen, C., A. Hilbeck, R. Howald, and W. Nentwig. 2003b. Effects of transgenic Bt corn litter on the earthworm Lumbricus terrestris. Molecular Ecology 12:10771086.

Zwahlen, C., A. Hilbeck, and W. Nentwig. 2007. Field decomposition of transgenic Bt maize residue and the impact on nontarget soil invertebrates. Plant and Soil 300:245-257. 


\section{Chapter 2. References}

Al-Karaki G, McMichael B \& Zak J (2004) Field response of wheat to arbuscular mycorrhizal fungi and drought stress. Mycorrhiza 14: 263-269.

Avio L, Cristani C, Strani P \& Giovannetti M (2009) Genetic and phenotypic diversity of geographically different isolates of Glomus mosseae. Canadian Journal of Microbiology 55: 242-253.

Baumgarte S \& Tebbe CC (2005) Field studies on the environmental fate of the Cry1 Ab Bt-toxin produced by transgenic maize (MON810) and its effect on bacterial communities in the maize rhizosphere. Molecular Ecology 14: 2539-2551.

Bressan W, Siqueira JO, Vasconcellos CA \& Purcino AAC (2001) Mycorhizal fungi and phosphorus on growth, yield and nutrition of intercropped grain sorghum and soybean. Pesquisa Agropecuaria Brasileira 36: 315-323.

Castaldini M, Turrini A, Sbrana C, Benedetti A, Marchionni M, Mocali S, Fabiani A, Landi S, Santomassimo F, Pietrangeli B, Nuti MP, Miclaus N \& Giovannetti M (2005) Impact of Bt corn on rhizospheric and soil eubacterial communities and on beneficial mycorrhizal symbiosis in experimental microcosms. Applied and Environmental Microbiology 71: 6719-6729.

de Vaufleury A, Kramarz PE, Binet P, Cortet J, Caul S, Andersen MN, Plumey E, Coeurdassier M \& Krogh PH (2007) Exposure and effects assessments of Btmaize on non-target organisms (gastropods, microarthropods, mycorrhizal fungi) in microcosms. Pedobiologia 51: 185-194.

Douds DD, Nagahashi G, Reider C \& Hepperly PR (2007) Inoculation with arbuscular mycorrhizal fungi increases the yield of potatoes in a high $\mathrm{P}$ soil. Biological Agriculture \& Horticulture 25: 67-78.

Douds DD \& Reider C (2003) Inoculation with mycorrhizal fungi increases the yield of green peppers in a high P soil. Biological Agriculture \& Horticulture 21: 91-102.

EPA USEPA (2007) Pesticides: Regulating Biopesticides, Plant Incorporated Protectants, Current \& Previously Registered Secion 3 PIP Registrations. http://www.epa.gov/pesticides/biopesticides/pips/pip_list.htm

Ferreira L, Molina JC, Brasil C \& Andrade G (2003) Evaluation of Bacillus thuringiensis bioinsecticidal protein effects on soil microorganisms. Plant and Soil 256: 161168.

Galvez L, Douds DD, Drinkwater LE \& Wagoner P (2001) Effect of tillage and farming system upon VAM fungus populations and mycorrhizas and nutrient uptake of maize. Plant and Soil 228: 299-308.

Gosling P, Hodge A, Goodlass G \& Bending GD (2006) Arbuscular mycorrhizal fungi and organic farming. Agriculture Ecosystems \& Environment 113: 17-35.

Hamel C \& Strullu DG (2006) Arbuscular mycorrhizal fungi in field crop production: Potential and new direction. Canadian Journal of Plant Science 86: 941-950.

Hirsch AM (2004) Plant-microbe symbioses: A continuum from commensalism to 
parasitism. Symbiosis 37: 345-363.

Icoz I \& Stotzky G (2008) Fate and effects of insect-resistant Bt crops in soil ecosystems. Soil Biology \& Biochemistry 40: 559-586.

James C (2010) Global Status of Commercialized Biotech/GM Crops: 2009.In. Ithaca, NY: International Service for the Acquisition of Agri-Biotech Applications.

Johnson NC, Graham JH \& Smith FA (1997) Functioning of mycorrhizal associations along the mutualism-parasitism continuum. New Phytologist 135: 575-586.

Jones MD \& Smith SE (2004) Exploring functional definitions of mycorrhizas: Are mycorrhizas always mutualisms? Canadian Journal of Botany-Revue Canadienne De Botanique 82: 1089-1109.

Karagiannidis N \& Hadjisavva-Zinoviadi S (1998) The mycorrhizal fungus Glomus mosseae enhances growth, yield and chemical composition of a durum wheat variety in 10 different soils. Nutrient Cycling in Agroecosystems 52: 1-7.

Knox OGG, Nehl DB, Mor T, Roberts GN \& Gupta V (2008) Genetically modified cotton has no effect on arbuscular mycorrhizal colonisation of roots. Field Crops Research 109: 57-60.

Lekberg Y, Koide RT \& Twomlow SJ (2008) Effect of agricultural management practices on arbuscular mycorrhizal fungal abundance in low-input cropping systems of southern Africa: a case study from Zimbabwe. Biology and Fertility of Soils 44: 917-923.

Mason MV (2010) Global Crop Unit Leader - Large Seeded Vegetables, Syngenta.In. Boise, ID.

McGonigle TP, Miller MH, Evans DG, Fairchild GL \& Swan JA (1990) A New Method Which Gives an Objective-Measure of Colonization of Roots by Vesicular Arbuscular Mycorrhizal Fungi. New Phytologist 115: 495-501.

Newman EI \& Reddell P (1987) The distribution of mycorrhizas among families of vascular plants. New Phytologist 106: 745-751.

Phillips JM \& Hayman DS (1970) Improved procedures for clearing roots and staining parasitic and vesicular-arbuscular mycorrhizal fungi for rapid assessment of infection. Transactions of the the British Mycological Society 55: 158-160.

Porra RJ (2002) The chequered history of the development and use of simultaneous equations for the accurate determination of chlorophylls a and b. Photosynthesis Research 73: 149-156.

Porra RJ, Thompson WA \& Kriedemann PE (1989) Determination of Accurate Extinction Coefficients and Simultaneous-Equations for Assaying Chlorophyll-a and Chlorophyll-B Extracted with 4 Different Solvents - Verification of the Concentration of Chlorophyll Standards by Atomic-Absorption Spectroscopy. Biochimica Et Biophysica Acta 975: 384-394.

Rosendahl S, McGee P \& Morton JB (2009) Lack of global population genetic differentiation in the arbuscular mycorrhizal fungus Glomus mosseae suggests a 
recent range expansion which may have coincided with the spread of agriculture. Molecular Ecology 18: 4316-4329.

Saxena D \& Stotzky G (2001) Bacillus thuringiensis (Bt) toxin released from root exudates and biomass of Bt corn has no apparent effect on earthworms, nematodes, protozoa, bacteria, and fungi in soil. Soil Biology \& Biochemistry 33: 1225-1230.

Sheng M, Tang M, Chen H, Yang BW, Zhang FF \& Huang YH (2008) Influence of arbuscular mycorrhizae on photosynthesis and water status of maize plants under salt stress. Mycorrhiza 18: 287-296.

Smith SE \& Read DJ (1997) Mycorrhizal Symbiosis. San Diego, CA: Academic Press. Thies JE \& Devare MH (2007) An ecological assessment of transgenic crops. Journal of Development Studies 43: 97-129.

Trappe JM (1987) Phylogenetic and ecologic aspects of mycotrophy in the angiosperms from an evolutionary standpoint. . Boca Raton, FL, USA: CRC Press.

Turrini A, Sbrana C, Nuti MP, Pietrangeli BM \& Giovannetti M (2004) Development of a model system to assess the impact of genetically modified corn and aubergine plants on arbuscular mycorrhizal fungi. Plant and Soil 266: 69-75.

USDA (2008) Adoption of Genetically Engineered Crops in the U.S.: Corn Varieties. http://www.ers.usda.gov/Data/BiotechCrops/ExtentofAdoptionTable1.htm 


\section{Chapter 3. References}

Burleigh, S. H., T. CAVAgnaro, AND I. JaKobSEN. 2002. Functional diversity of arbuscular mycorrhizas extends to the expression of plant genes involved in $\mathrm{P}$ nutrition. Journal of Experimental Botany 53: 1593-1601.

Castaldini, M., A. Turrini, C. Sbrana, A. Benedetti, M. Marchionni, S. Mocali, A. Fabiani, S. Landi, F. SAntomassimo, B. Pietrangeli, M. P. Nuti, N. MiClAUS, AND M. GIOVANNETTI. 2005. Impact of $B t$ corn on rhizospheric and soil eubacterial communities and on beneficial mycorrhizal symbiosis in experimental microcosms. Applied and Environmental Microbiology 71: 67196729.

Cheeke, T. E., B. A. Pace, T. N. Rosenstiel, AND M. B. Cruzan. 2011. The influence of fertilizer level and spore density on arbuscular mycorrhizal colonization of transgenic Bt 11 maize (Zea mays) in experimental microcosms. FEMS Microbiology Ecology 75: 304-312.

de Vaufleury, A., P. E. Kramarz, P. Binet, J. Cortet, S. Caul, M. N. Andersen, E. Plumey, M. Coeurdassier, AND P. H. Krogh. 2007. Exposure and effects assessments of $B t$-maize on non-target organisms (gastropods, microarthropods, mycorrhizal fungi) in microcosms. Pedobiologia 51: 185-194.

Devare, M., L. M. Londono-R, AND J. E. ThIES. 2007. Neither transgenic $B t$ maize (MON863) nor tefluthrin insecticide adversely affect soil microbial activity or biomass: A 3-year field analysis. Soil Biology \& Biochemistry 39: 2038-2047.

Donegan, K. K., C. J. Palm, V. J. Fieland, L. A. Porteous, L. M. Ganio, D. L. SCHALlER, L. Q. BuCAO, AND R. J. SEIDLER. 1995. Changes in levels, species and DNA fingerprints of soil-microorganisms associated with cotton expressing the Bacillus thuringiensis var kurstaki endotoxin. Applied Soil Ecology 2: 111124.

Douds, D. D., L. GALveZ, G. BECARD, AND Y. KAPUlNIK. 1998. Regulation of arbuscular mycorrhizal development by plant host and fungus species in alfalfa. New Phytologist 138: 27-35.

DUNFIELD, K. E. AND J. J. GERMIDA. 2003. Seasonal changes in the rhizosphere microbial communities associated with field-grown genetically modified canola (Brassica napus). Applied and Environmental Microbiology 69: 7310-7318.

DUNFIELD, K. E. AND J. J. GERMIDA. 2004. Impact of genetically modified crops on soil- and plant-associated microbial communities. Journal of Environmental Quality 33: 806-815.

EPA, United States EnVIRonmental Protection Agency. 2011. Pesticides:

Regulating Biopesticides, Plant Incorporated Protectants, Current \& Previously Registered Secion 3 PIP Registrations. http://www.epa.gov/pesticides/biopesticides/pips/pip_list.htm.

FEDERICI, B. A. 2002. Case study: Bt crops, a novel mode of insect control. In K. T. 
Atherton [ed.], Genetically Modified Crops: Assessing Safety, 164-200. Taylor \& Francis Inc., New York, NY.

Ferreira, L., J. C. Molina, C. Brasil, AND G. Andrade. 2003. Evaluation of Bacillus thuringiensis bioinsecticidal protein effects on soil microorganisms. Plant and Soil 256: 161-168.

FLORES, S., D. SAXENA, AND G. STOTZKY. 2005. Transgenic $B t$ plants decompose less in soil than non-Bt plants. Soil Biology \& Biochemistry 37: 1073-1082.

GALVEZ, L., D. D. DoudS, L. E. DrinKWATER, AND P. WAGONER. 2001. Effect of tillage and farming system upon VAM fungus populations and mycorrhizas and nutrient uptake of maize. Plant and Soil 228: 299-308.

GaO, X. P., T. W. Kuyper, C. Q. Zou, F. S. Zhang, AND E. HofFland. 2007. Mycorrhizal responsiveness of aerobic rice genotypes is negatively correlated with their zinc uptake when nonmycorrhizal. Plant and Soil 290: 283-291.

Gosling, P., A. Hodge, G. Goodlass, AND G. D. Bending. 2006. Arbuscular mycorrhizal fungi and organic farming. Agriculture Ecosystems \& Environment 113: 17-35.

Graham, J. H., AND L. K. AвBOTT. 2000. Wheat responses to aggressive and nonaggressive arbuscular mycorrhizal fungi. Plant and Soil 220: 207-218.

GRIFFITHS, B. S., S. CAUL, J. THOMPSON, C. A. HACKETT, J. CORTET, C. PERNIN, AND P. H. KROGH. 2008. Soil microbial and faunal responses to herbicide tolerant maize and herbicide in two soils. Plant and Soil 308: 93-103.

Hetrick, B. A. D., G. W. T. WiLson, AND T. S. CoX. 1992. Mycorrhizal dependence of modern wheat varieties, landraces, and ancestors. Canadian Journal of BotanyRevue Canadienne De Botanique 70: 2032-2040.

HIRSCH, A. M. 2004. Plant-microbe symbioses: A continuum from commensalism to parasitism. Symbiosis 37: 345-363.

ICOZ, I., AND G. STOTZKY. 2008a. Fate and effects of insect-resistant $B t$ crops in soil ecosystems. Soil Biology \& Biochemistry 40: 559-586. . 2008b. Cry3Bb1 protein from Bacillus thuringiensis in root exudates and biomass of transgenic corn does not persist in soil. Transgenic Research 17: 609620.

JAMES, C. 2010. Global Status of Commercialized Biotech/GM Crops: 2009. International Service for the Acquisition of Agri-Biotech Applications, Ithaca, NY, ISAAA Brief No. 41.

JANSA, J., F. A. SMITH, AND S. E. SMITH. 2008. Are there benefits of simultaneous root colonization by different arbuscular mycorrhizal fungi? New Phytologist 177: 779-789.

Johnson, N. C., J. H. GRAHAM, AND F. A. SMITH. 1997. Functioning of mycorrhizal associations along the mutualism-parasitism continuum. New Phytologist 135: 575-586. 
JONES, M. D., AND S. E. SMITH. 2004. Exploring functional definitions of mycorrhizas: Are mycorrhizas always mutualisms? Canadian Journal of Botany-Revue Canadienne De Botanique 82: 1089-1109.

Kaeppler, S. M., J. L. PArke, S. M. Mueller, L. Senior, C. Stuber, AND W. F. TRACY. 2000. Variation among maize inbred lines and detection of quantitative trait loci for growth at low phosphorus and responsiveness to arbuscular mycorrhizal fungi. Crop Science 40: 358-364.

KIERS, E. T., S. A. WEST, AND R. F. DENISON. 2002. Mediating mutualisms: farm management practices and evolutionary changes in symbiont co-operation. Journal of Applied Ecology 39: 745-754.

Knox, O. G. G., D. B. Nehl, T. Mor, G. N. RoberTs, AND V. GuPTA. 2008. Genetically modified cotton has no effect on arbuscular mycorrhizal colonisation of roots. Field Crops Research 109: 57-60.

Koskella, J., AND G. STOTZKy. 2002. Larvicidal toxins from Bacillus thuringiensis subspp. kurstaki, morrisoni (strain tenebrionis), and israelensis have no microbicidal or microbiostatic activity against selected bacteria, fungi, and algae in vitro. Canadian Journal of Microbiology 48: 262-267.

KOWALCHUK, G. A., M. BRUINSMA, AND J. A. VAN VEEN. 2003. Assessing responses of soil microorganisms to GM plants. Trends in Ecology and Evolution 18: 403-410.

KROGH, P. H., B. GRIFFITHS, D. DEMSAR, M. BOHANEC, M. DEBELJAK, M. N. ANDERSEN, C. SAUSSE, A. N. E. BIRCH, S. CAUL, M. HOLMSTRUP, L. H. HECKMANN, AND J. CORTET. 2007. Responses by earthworms to reduced tillage in herbicide tolerant maize and $B t$ maize cropping systems. Pedobiologia 51: 219-227.

LEe, L., D. SAXENA, AND G. StOTZKY. 2003. Activity of free and clay-bound insecticidal proteins from Bacillus thuringiensis subsp israelensis against the mosquito Culex pipiens. Applied and Environmental Microbiology 69: 41114115.

LEKBERG, Y., R. T. KoIDE, AND S. J. TwOMLOw. 2008. Effect of agricultural management practices on arbuscular mycorrhizal fungal abundance in low-input cropping systems of southern Africa: a case study from Zimbabwe. Biology and Fertility of Soils 44: 917-923.

LUNDGREN, J. G., A. J. GASSMANN, J. BERNAL, J. J. DUAN, AND J. RUBERSON. 2009. Ecological compatibility of GM crops and biological control. Crop Protection 28: 1017-1030.

McGonigle, T. P., M. H. Miller, D. G. Evans, G. L. Fairchild, AND J. A. Swan. 1990. A new method which gives an objective-measure of colonization of roots by vesicular arbuscular mycorrhizal fungi. New Phytologist 115: 495-501.

Morton, J. B. 2008. Professor and Chairman Plant Pathology and Environmental 
Microbiology West Virginia University, Morgantown, WV.

NAEF, A., T. ZESIGER, AND G. DEFAGO. 2006. Impact of transgenic $B t$ maize residues on the mycotoxigenic plant pathogen Fusarium graminearum and the biocontrol agent Trichoderma atroviride. Journal of Environmental Quality 35: 1001-1009.

PHILlIPS, J. M., AND D. S. HAYMAN. 1970. Improved procedures for clearing roots and staining parasitic and vesicular-arbuscular mycorrhizal fungi for rapid assessment of infection. Transactions of the British Mycological Society 55: 158-160.

SANCHIS, V. 2011. From microbial sprays to insect-resistant transgenic plants: history of the biospesticide Bacillus thuringiensis. A review. Agronomy for Sustainable Development 31: 217-231.

SAwERS, R. J. H., C. GuTJAHR, AND U. PASZKOWSKI. 2008. Cereal mycorrhiza: an ancient symbiosis in modern agriculture. Trends in Plant Science 13: 93-97.

SAXENA, D., AND G. STOTZKY. 2000. Insecticidal toxin from Bacillus thuringiensis is released from roots of transgenic Bt corn in vitro and in situ. FEMS Microbiology Ecology 33: 35-39.

SAXENA, D., S. Flores, AND G. STOTZKY. 2002. Bt toxin is released in root exudates from 12 transgenic corn hybrids representing three transformation events. Soil Biology \& Biochemistry 34: 133-137.

Schnepf, E., N. Crickmore, J. VAn Rie, D. Lereclus, J. BAum, J. Feitelson, D. R. ZEIGLER, AND D. H. DEAN. 1998. Bacillus thuringiensis and its pesticidal crystal proteins. Microbiology and Molecular Biology Reviews 62: 775-806.

Schultz, P. A., R. M. Miller, J. D. Jastrow, C. V. Rivetta, AND J. D. Bever. 2001. Evidence of a mycorrhizal mechanism for the adaptation of Andropogon gerardii (Poaceae) to high- and low-nutrient prairies. American Journal of Botany 88: 1650-1656.

SEIFERT, E. K., J. D. BEVER, AND J. L. MARON. 2009. Evidence for the evolution of reduced mycorrhizal dependence during plant invasion. Ecology 90: 1055-1062.

Sheng, M., M. TAng, H. Chen, B. W. YAng, F. F. Zhang, AND Y. H. HuAng. 2008. Influence of arbuscular mycorrhizae on photosynthesis and water status of maize plants under salt stress. Mycorrhiza 18: 287-296.

Sheveleva, E. V., S. Marquez, W. Chmara, A. Zegeer, R. G. Jensen, AND H. J. BOHNERT. 1998. Sorbitol-6-phosphate dehydrogenase expression in transgenic tobacco - High amounts of sorbitol lead to necrotic lesions. Plant Physiology 117: 831-839.

SICILIANO, S. D. AND J. J. GERMIDA. 1999. Taxonomic diversity of bacteria associated with the roots of field-grown transgenic Brassica napus cv. Quest, compared to the non-transgenic B. napus cv. Excel and B. rapa cv. Parkland. FEMS Microbiology Ecology 29: 263-272.

Smith, S. E., AND D. J. READ. 2008. Mycorrhizal Symbiosis. Academic Press, London.

SMith, S. E., F. A. SMITH, AND I. JAKOBSEN. 2003. Mycorrhizal fungi can dominate 
phosphate supply to plants irrespective of growth responses. Plant Physiology 133: 16-20.

.2004. Functional diversity in arbuscular mycorrhizal (AM) symbioses: the contribution of the mycorrhizal P uptake pathway is not correlated with mycorrhizal responses in growth or total P uptake. New Phytologist 162: 511-524. STOTZKY, G. 2002. Release, persistence, and biological activity in soil of insecticidal proteins from Bacillus thuringiensis. In D. K. Letourneau and B. E. Burrows [eds.], Genetically Engineered Organisms: Assessing Environmental and Human Health Effects, 187-222. CRC Press, Boca Raton, FL.

Turrini, A., C. Sbrana, M. P. Nuti, B. M. Pietrangeli, AND M. Giovannetti. 2004. Development of a model system to assess the impact of genetically modified corn and aubergine plants on arbuscular mycorrhizal fungi. Plant and Soil 266: 69-75.

USDA, United States Department of Agriculture . 2010. Adoption of Genetically Engineered Crops in the U.S.: Corn Varieties. http://www.ers.usda.gov/Data/BiotechCrops/ExtentofAdoptionTable1.htm

WANG, W. X., B. VINOCUR, AND A. ALTMAN. 2003. Plant responses to drought, salinity and extreme temperatures: towards genetic engineering for stress tolerance.

Planta 218: 1-14. 


\section{Chapter 4. References}

1. USDA (United States Department of Agriculture). 2012. Adoption of Genetically Engineered Crops in the U.S.: Corn Varieties. http://www.ers.usda.gov/Data/BiotechCrops/ExtentofAdoptionTable1.htm. Accesses 15 Nov. 2012.

2. James, C. 2012. Global Status of Commercialized Biotech/GM Crops: 2011. ISAAA Brief No. 43. International Service for the Acquisition of Agri-Biotech Applications. http://www.isaaa.org/resources/publications/briefs/43/default.asp. Accessed 15 Nov. 2012.

3. Icoz, I., and G. Stotzky. 2008. Fate and effects of insect-resistant $B t$ crops in soil ecosystems. Soil Biology \& Biochemistry 40:559-586.

4. Sanchis, V. 2011. From microbial sprays to insect-resistant transgenic plants: history of the biospesticide Bacillus thuringiensis. A review. Agronomy for Sustainable Development 31:217-231.

5. Federici, B. A. 1993. Insecticidal bacterial proteins identify the midgut epithelium as a source of nobel target sites for insect control. Archives of Insect Biochemistry and Physiology 22:357-371.

6. Bravo, A., S. S. Gill, and M. Soberon. 2007. Mode of action of Bacillus thuringiensis Cry and Cyt toxins and their potential for insect control. Toxicon 49:423-435.

7. Cheeke, T. E. 2012. Effects of the cultivation of genetically modified $B t$ crops on nontarget soil organisms, p. 153-227. In T. E. Cheeke, D. C. Coleman, and D. H. Wall (ed.), Microbial Ecology in Sustainable Agroecosystems. CRC Press, Boca Raton.

8. Smith, S. E., and D. J. Read. 2008. Mycorrhizal Symbiosis, 3rd ed. Academic Press, San Diego, CA.

9. Castaldini, M., A. Turrini, C. Sbrana, A. Benedetti, M. Marchionni, S. Mocali, A. Fabiani, S. Landi, F. Santomassimo, B. Pietrangeli, M. P. Nuti, N. Miclaus, and M. Giovannetti. 2005. Impact of $B t$ corn on rhizospheric and soil eubacterial communities and on beneficial mycorrhizal symbiosis in experimental microcosms. Applied and Environmental Microbiology 71:6719-6729.

10. Cheeke, T. E., B. A. Pace, T. N. Rosenstiel, and M. B. Cruzan. 2011. The influence of fertilizer level and spore density on arbuscular mycorrhizal colonization of transgenic Bt 11 maize (Zea mays) in experimental microcosms. FEMS Microbiology Ecology 75:304-312.

11. Cheeke, T. E., T. N. Rosenstiel, and M. B. Cruzan. 2012. Evidence of reduced arbuscular mycorhizal fungal colonizaiton in multiple lines of $B t$ maize. American Journal of Botany 99:700-707.

12. Turrini, A., C. Sbrana, M. P. Nuti, B. M. Pietrangeli, and M. Giovannetti. 2004. Development of a model system to assess the impact of genetically 
modified corn and aubergine plants on arbuscular mycorrhizal fungi. Plant and Soil 266:69-75.

13. Broeckling, C. D., A. K. Broz, J. Bergelson, D. K. Manter, and J. M. Vivanco. 2008. Root exudates regulate soil fungal community composition and diversty. Applied and Environmental Microbiology 74:738-744.

14. Bucking, H., J. Abubaker, M. Govindarajulu, M. Tala, P. E. Pfeffer, G. Nagahashi, P. Lammers, and Y. Shachar-Hill. 2008. Root exudates stimulate the uptake and metabolism of organic carbon in germinating spores of Glomus intraradices. New Phytologist 180:684-695.

15. Hinsinger, P., G. R. Gobran, P. J. Gregory, and W. W. Wenzel. 2005. Rhizosphere geometry and heterogeneity arising from root-mediated physical and chemical processes. New Phytologist 168:293-303.

16. Kaeppler, S. M., J. L. Parke, S. M. Mueller, L. Senior, C. Stuber, and W. F. Tracy. 2000. Variation among maize inbred lines and detection of quantitative trait loci for growth at low phosphorus and responsiveness to arbuscular mycorrhizal fungi. Crop Science 40:358-364.

17. Garcia-Rodriguez, S., C. Azcon-Aguilar, and N. Ferrol. 2007. Transcriptional regulation of host enzymes involved in the cleavage of sucrose during arbuscular mycorrhizal symbiosis. Physiologia Plantarum 129:737-746.

18. Schaarschmidt, S., J. Kopka, J. Ludwig-Muller, and B. Hause. 2007. Regulation of arbuscular mycorrhization by apoplastic invertases: Enhanced invertase activity in the leaf apoplast affects the symbiotic interaction. Plant Journal 51:390-405.

19. Schaarschmidt, S., T. Roitsch, and B. Hause. 2006. Arbuscular mycorrhiza induces gene expression of the apoplastic invertase LIN6 in tomato (Lycopersicon esculentum) roots. Journal of Experimental Botany 57:4015-4023.

20. Bucking, H., and Y. Shachar-Hill. 2005. Phosphate uptake, transport and transfer by the arbuscular mycorrhizal fungus Glomus intraradices is stimulated by increased carbohydrate availability. New Phytologist 165:899-912.

21. Doidy, J., D. van Tuinen, O. Lamotte, M. Corneillat, G. Alcaraz, and D. Wipf. 2012. The Medicago truncatula sucrose transporter family: Characterization and implication of key members in carbon partitioning towards arbuscular mycorrhizal fungi. Molecular Plant 5:1346-1358.

22. Blee, K. A., and A. J. Anderson. 1998. Regulation of arbuscule formation by carbon in the plant. Plant Journal 16:523-530.

23. de Vaufleury, A., P. E. Kramarz, P. Binet, J. Cortet, S. Caul, M. N. Andersen, E. Plumey, M. Coeurdassier, and P. H. Krogh. 2007. Exposure and effects assessments of Bt-maize on non-target organisms (gastropods, microarthropods, mycorrhizal fungi) in microcosms. Pedobiologia 51:185-194.

24. Tan, F. X., J. W. Wang, Z. N. Chen, Y. J. Feng, G. L. Chi, and S. U. Rehman. 
2011. Assessment of the arbuscular mycorrhizal fungal community in roots and rhizosphere soils of $B t$ corn and their non- $B t$ isolines. Soil Biology \& Biochemistry 43:2473-2479.

25. Verbruggen, E., E. E. Kuramae, R. Hillekens, M. de Hollander, E. T. Kiers, W. F. M. Roling, G. A. Kowalchuk, and M. G. A. van der Heijden. 2012.

Testing potential effects of maize expressing the Bacillus thuringiensis Cry $1 \mathrm{Ab}$ endotoxin ( $B t$ maize) on mycorrhizal fungal communities via DNA- and RNAbased pyrosequencing and molecular fingerprinting. Applied and Environmental Microbiology 78:7384-7392.

26. Knox, O. G. G., D. B. Nehl, T. Mor, G. N. Roberts, and V. Gupta. 2008. Genetically modified cotton has no effect on arbuscular mycorrhizal colonisation of roots. Field Crops Research 109:57-60.

27. NOAA (National Oceanic and Atmospheric Administration). 2012. National Climatic Data Center. http://ncdc.noaa.gov/cdo-web. Accessed 15 Nov. 2012.

28. Natural Resources Conservation Service, USDA. 2012. Soil Survey Geographic (SSURGO) Database for Corvallis, Oregon, vol. 2012. USDA, NRCS. http://soildatamart.nrcs.usda.gov. Accessed 15 Nov. 2012.

29. Gerdemann, J., and T. H. Nicolson. 1963. Spores of mycorrhizal Endogone species extracted from soil by wet sieving and decanting. Transactions of the British Mycological Society 46:235-244.

30. Walker, C., C. W. Mize, and H. S. McNabb Jr. 1982. Populations of endogonaceous fungi at two locations in central Iowa. Canadian Journal of Botany 60:2518-2529.

31. McKenney, M. C., and D. L. Lindsey. 1987. Improved method for quantifying endomycorrhizal fungi spores from soil. Mycologia 79:779-782.

32. Phillips, J. M., and D. S. Hayman. 1970. Improved procedures for clearing roots and staining parasitic and vesicular-arbuscular mycorrhizal fungi for rapid assessment of infection. Transactions of the the British Mycological Society 55:158-160.

33. McGonigle, T. P., M. H. Miller, D. G. Evans, G. L. Fairchild, and J. A. Swan. 1990. A new method which gives an objective-measure of colonization of roots by vesicular arbuscular mycorrhizal fungi. New Phytologist 115:495-501.

34. Isobe, K., H. Sugimura, T. Maeshima, and R. Ishii. 2008. Distribution of arbuscular mycorrhizal fungi in upland field soil of Japan - 2. Spore density of arbuscular mycorrhizal fungi and infection ratio in soybean and maize fields. Plant Production Science 11:171-177.

35. Kabir, Z., I. P. O'Halloran, P. Widden, and C. Hamel. 1998. Vertical distribution of arbuscular mycorrhizal fungi under corn (Zea mays L.) in no-till and conventional tillage systems. Mycorrhiza 8:53-55.

36. Oehl, F., E. Sieverding, K. Ineichen, E. A. Ris, T. Boller, and A. Wiemken. 
2005. Community structure of arbuscular mycorrhizal fungi at different soil depths in extensively and intensively managed agroecosystems. New Phytologist 165:273-283.

37. Helgason, T., T. J. Daniell, R. Husband, A. H. Fitter, and J. P. W. Young. 1998. Ploughing up the wood-wide web? Nature 394:431-431.

38. Oehl, F., E. Sieverding, K. Ineichen, P. Mader, T. Boller, and A. Wiemken. 2003. Impact of land use intensity on the species diversity of arbuscular mycorrhizal fungi in agroecosystems of Central Europe. Applied and Environmental Microbiology 69:2816-2824.

39. Johnson, N. C., J. H. Graham, and F. A. Smith. 1997. Functioning of mycorrhizal associations along the mutualism-parasitism continuum. New Phytologist 135:575-586.

40. Johnson, N. C., and J. H. Graham. 2013. The continuum concept remains a useful framework for studying mycorrhizal functioning. Plant and Soil 363:411419.

41. Smith, F. A., and S. E. Smith. 2013. How useful is the mutualism-parasitism continuum of arbuscular mycorrhizal functioning? Plant and Soil 363:7-18.

42. Grigera, M. S., R. A. Drijber, R. H. Shores-Morrow, and B. J. Wienhold. 2007. Distribution of the arbuscular mycorrhizal biomarker C16 : 1cis11 among neutral, glyco and phospholipids extracted from soil during the reproductive growth of corn. Soil Biology \& Biochemistry 39:1589-1596.

43. Grigera, M. S., R. A. Drijber, and B. J. Wienhold. 2007. Increased abundance of arbuscular mycorrhizal fungi in soil coincides with the reproductive stages of maize. Soil Biology \& Biochemistry 39:1401-1409.

44. Blackmer, T. M., and J. S. Schepers. 1995. Use of a chlorophyll meter to monitor nitrogen status and schedule fertigation for corn. Journal of Production Agriculture 8:56-60.

45. Wood, C. W., D. W. Reeves, R. R. Duffield, and K. L. Edmisten. 1992. Field chlorophyll measurements for evaluation of corn nitrogen status. Journal of Plant Nutrition 15:487-500.

46. Bullock, D. G., and D. S. Anderson. 1998. Evaluation of the Minolta SPAD-502 chlorophyll meter for nitrogen management in corn. Journal of Plant Nutrition 21:741-755.

47. Hodge, A. 2004. The plastic plant: root responses to heterogeneous supplies of nutrients. New Phytologist 162:9-24.

48. Johnson, N. C., D. L. Rowland, L. Corkidi, L. M. Egerton-Warburton, and E. B. Allen. 2003. Nitrogen enrichment alters mycorrhizal allocation at five mesic to semiarid grasslands. Ecology 84:1895-1908.

49. Schreiner, R. P., and K. L. Mihara. 2009. The diversity of arbuscular mycorrhizal fungi amplified from grapevine roots (Vitis vinifera L.) in Oregon 
vineyards is seasonally stable and influenced by soil and vine age. Mycologia 101:599-611.

50. Ryan, M. H., and J. H. Graham. 2002. Is there a role for arbuscular mycorrhizal fungi in production agriculture? Plant and Soil 244:263-271.

51. Harrier, L. A., and C. A. Watson. 2003. The role of arbuscular mycorrhizal fungi in sustainable cropping systems, p. 185-225, Advances in Agronomy, vol 79.

52. Hooker, J. E., and K. E. Black. 1995. Arbuscular mycorrhizal fungi as components of sustainable soil-plant systems. Critical Reviews in Biotechnology 15:201-212.

53. Jeffries, P., S. Gianinazzi, S. Perotto, K. Turnau, and J. M. Barea. 2003. The contribution of arbuscular mycorrhizal fungi in sustainable maintenance of plant health and soil fertility. Biology and Fertility of Soils 37:1-16.

54. Gavito, M. E., and M. H. Miller. 1998. Changes in mycorrhiza development in maize induced by crop management practices. Plant and Soil 198:185-192.

55. Johnson, N. C., F. L. Pfleger, R. K. Crookston, S. R. Simmons, and P. J. Copeland. 1991. Vesicular arbuscular mycorrhizas respond to corn and soybean cropping history. New Phytologist 117:657-663.

56. McCain, K. N. S., G. W. T. Wilson, and J. M. Blair. 2011. Mycorrhizal suppression alters plant productivity and forb establishment in a grass-dominated prairie restoration. Plant Ecology 212:1675-1685.

57. Middleton, E. L., and J. D. Bever. 2012. Inoculation with a native soil community advances succession in a grassland restoration. Restoration Ecology 20:218-226.

58. Alguacil, M. M., E. Lumini, A. Roldan, J. R. Salinas-Garcia, P. Bonfante, and V. Bianciotto. 2008. The impact of tillage practices on arbuscular mycorrhizal fungal diversity in subtropical crops. Ecological Applications 18:527-536.

59. Douds, D. D., L. Galvez, R. R. Janke, and P. Wagoner. 1995. Effect of tillage and farming system upon populations and distribution of vesicular-arbuscular mycorrhizal fungi. Agriculture Ecosystems \& Environment 52:111-118.

60. Galvez, L., D. D. Douds, L. E. Drinkwater, and P. Wagoner. 2001. Effect of tillage and farming system upon VAM fungus populations and mycorrhizas and nutrient uptake of maize. Plant and Soil 228:299-308.

61. Lekberg, Y., R. T. Koide, and S. J. Twomlow. 2008. Effect of agricultural management practices on arbuscular mycorrhizal fungal abundance in low-input cropping systems of southern Africa: A case study from Zimbabwe. Biology and Fertility of Soils 44:917-923.

62. Johnson, N. C. 1993. Can fertilization of soil select less mutualistic mycorrhizae. Ecological Applications 3:749-757. 
63. Martinez, T. N., and N. C. Johnson. 2010. Agricultural management influences propagule densities and functioning of arbuscular mycorrhizas in low- and highinput agroecosystems in arid environments. Applied Soil Ecology 46:300-306. 


\section{Chapter 5. References}

Akiyama, K., K. Matsuzaki, and H. Hayashi. 2005. Plant sesquiterpenes induce hyphal branching in arbuscular mycorrhizal fungi. Nature 435:824-827.

Auge, R. M. 2001. Water relations, drought and vesicular-arbuscular mycorrhizal symbiosis. Mycorrhiza 11:3-42.

Bainard, L. D., P. D. Brown, and M. K. Upadhyaya. 2009. Inhibitory effect of tall hedge mustard (Sisymbrium loeselii) allelochemicals on rangeland plants and arbuscular mycorrhizal fungi. Weed Science 57:386-393.

Bais, H. P., T. L. Weir, L. G. Perry, S. Gilroy, and J. M. Vivanco. 2006. The role of root exudates in rhizosphere interations with plants and other organisms. Annual Review of Plant Biology 57:233-266.

Barzana, G., R. Aroca, J. A. Paz, F. Chaumont, M. C. Martinez-Ballesta, M. Carvajal, and J. M. Ruiz-Lozano. 2012. Arbuscular mycorrhizal symbiosis increases relative apoplastic water flow in roots of the host plant under both well-watered and drought stress conditions. Annals of Botany 109:1009-1017.

Bever, J. D. 2002. Host-specificity of AM fungal population growth rates can generate feedback on plant growth. Plant and Soil 244:281-290.

Bever, J. D., T. G. Platt, and E. R. Morton. 2012. Microbial population and community dynamics on plant roots and their feedbacks on plant communities. Pages 265-283

in S. Gottesman, C. S. Harwood, and O. Schneewind, editors. Annual Review of Microbiology, Vol 66.

Bever, J. D., S. C. Richardson, B. M. Lawrence, J. Holmes, and M. Watson. 2009. Preferential allocation to beneficial symbiont with spatial structure maintains mycorrhizal mutualism. Ecology Letters 12:13-21.

Bever, J. D., K. M. Westover, and J. Antonovics. 1997. Incorporating the soil community into plant population dynamics: the utility of the feedback approach. Journal of Ecology 85:561-573.

Bravo, A., S. S. Gill, and M. Soberon. 2007. Mode of action of Bacillus thuringiensis Cry and Cyt toxins and their potential for insect control. Toxicon 49:423-435.

Broeckling, C. D., A. K. Broz, J. Bergelson, D. K. Manter, and J. M. Vivanco. 2008. Root exudates regulate soil fungal community composition and diversity. Applied and Environmental Microbiology 74:738-744.

Bullock, D. G. 1992. Crop-rotation. Critical Reviews in Plant Sciences 11:309-326.

Callaway, R. M., D. Cipollini, K. Barto, G. C. Thelen, S. G. Hallett, D. Prati, K. Stinson, and J. Klironomos. 2008. Novel weapons: Invasive plant suppresses fungal mutualists in America but not in its native Europe. Ecology 89:1043-1055.

Castaldini, M., A. Turrini, C. Sbrana, A. Benedetti, M. Marchionni, S. Mocali, A. Fabiani, S. Landi, F. Santomassimo, B. Pietrangeli, M. P. Nuti, N. Miclaus, and M. Giovannetti. 2005. Impact of Bt corn on rhizospheric and soil eubacterial communities and on beneficial mycorrhizal symbiosis in experimental 
microcosms. Applied and Environmental Microbiology 71:6719-6729.

Cheeke, T. E. 2012. Effects of the cultivation of genetically modified $B t$ crops on nontarget soil organisms. Pages 153-227 in T. E. Cheeke, D. C. Coleman, and D. H. Wall, editors. Microbial Ecology in Sustainable Agroecosystems. CRC Press, Boca Raton.

Cheeke, T. E., M. B. Cruzan, and T. N. Rosenstiel. 2013. A field evaluation of arbuscular mycorrhizal fungal colonization in $B t$ and non- $B t$ maize. Applied and Environmental Microbiology 79:4078-4086.

Cheeke, T. E., B. A. Pace, T. N. Rosenstiel, and M. B. Cruzan. 2011. The influence of fertilizer level and spore density on arbuscular mycorrhizal colonization of transgenic Bt 11 maize (Zea mays) in experimental microcosms. FEMS Microbiology Ecology 75:304-312.

Cheeke, T. E., T. N. Rosenstiel, and M. B. Cruzan. 2012. Evidence of reduced arbuscular mycorhizal fungal colonizaiton in multiple lines of $B t$ maize. American Journal of Botany 99:700-707.

de Vaufleury, A., P. E. Kramarz, P. Binet, J. Cortet, S. Caul, M. N. Andersen, E. Plumey, M. Coeurdassier, and P. H. Krogh. 2007. Exposure and effects assessments of Btmaize on non-target organisms (gastropods, microarthropods, mycorrhizal fungi) in microcosms. Pedobiologia 51:185-194.

Dively, G. P., R. Rose, M. K. Sears, R. L. Hellmich, D. E. Stanley-Horn, D. D. Calvin, J. M. Russo, and P. L. Anderson. 2004. Effects on monarch butterfly larvae (Lepidoptera : Danaidae) after continuous exposure to Cry1 Ab-expressing corn during anthesis. Environmental Entomology 33:1116-1125.

Douds, D. D., L. Galvez, R. R. Janke, and P. Wagoner. 1995. Effect of tillage and farming system upon populations and distribution of vesicular-arbuscular mycorrhizal fungi. Agriculture Ecosystems \& Environment 52:111-118.

Federici, B. A. 1993. Insecticidal bacterial proteins identify the midgut epithelium as a source of nobel target sites for insect control. Archives of Insect Biochemistry and Physiology 22:357-371.

Galvez, L., D. D. Douds, L. E. Drinkwater, and P. Wagoner. 2001. Effect of tillage and farming system upon VAM fungus populations and mycorrhizas and nutrient uptake of maize. Plant and Soil 228:299-308.

Gavito, M. E. and M. H. Miller. 1998. Changes in mycorrhiza development in maize induced by crop management practices. Plant and Soil 198:185-192.

Gerdemann, J. and T. H. Nicolson. 1963. Spores of mycorrhizal Endogone species extracted from soil by wet sieving and decanting. Transactions of the British Mycological Society 46:235-244.

Harrier, L. A. and C. A. Watson. 2003. The role of arbuscular mycorrhizal fungi in sustainable cropping systems. Pages 185-225 Advances in Agronomy, Vol 79.

Hooker, J. E. and K. E. Black. 1995. Arbuscular mycorrhizal fungi as components of 
sustainable soil-plant systems. Critical Reviews in Biotechnology 15:201-212.

Hoss, S., M. Arndt, S. Baurngarte, C. C. Tebbe, H. T. Nguyen, and J. A. Jehle. 2008.

Effects of transgenic corn and CrylAb protein on the nematode, Caenorhabditis elegans. Ecotoxicology and Environmental Safety 70:334-340.

Icoz, I. and G. Stotzky. 2008. Fate and effects of insect-resistant Bt crops in soil ecosystems. Soil Biology \& Biochemistry 40:559-586.

Jakobsen, I., L. K. Abbott, and A. D. Robson. 1992. External hyphae of vesiculararbuscular mycorrhizal fungi associated with Trifolium-subterraneum L .1. Spread of hyphae and phosphorus inflow into roots New Phytologist 120:371380.

Jansa, J., A. Mozafar, and E. Frossard. 2005. Phosphorus acquisition strategies within arbuscular mycorrhizal fungal community of a single field site. Plant and Soil 276:163-176.

Jeffries, P., S. Gianinazzi, S. Perotto, K. Turnau, and J. M. Barea. 2003. The contribution of arbuscular mycorrhizal fungi in sustainable maintenance of plant health and soil fertility. Biology and Fertility of Soils 37:1-16.

Johnson, N. C., F. L. Pfleger, R. K. Crookston, S. R. Simmons, and P. J. Copeland. 1991. Vesicular arbuscular mycorrhizas respond to corn and soybean cropping history. New Phytologist 117:657-663.

Johnson, N. C., D. L. Rowland, L. Corkidi, and E. B. Allen. 2008. Plant winners and losers during grassland $\mathrm{N}$-eutrophication differ in biomass allocation and mycorrhizas. Ecology 89:2868-2878.

Jung, S. C., A. Martinez-Medina, J. A. Lopez-Raez, and M. J. Pozo. 2012. Mycorrhizainduced resistance and priming of plant defenses. Journal of Chemical Ecology 38:651-664.

Kiers, E. T., M. Duhamel, Y. Beesetty, J. A. Mensah, O. Franken, E. Verbruggen, C. R. Fellbaum, G. A. Kowalchuk, M. M. Hart, A. Bago, T. M. Palmer, S. A. West, P. Vandenkoornhuyse, J. Jansa, and H. Bucking. 2011. Reciprocal rewards stabilize cooperation in the mycorrhizal symbiosis. Science 333:880-882.

Kinkel, L. L., M. G. Bakker, and D. C. Schlatter. 2011. A coevolutionary framework for managing disease-suppressive soils. Pages 47-67 in N. K. VanAlfen, G. Bruening, and J. E. Leach, editors. Annual Review of Phytopathology, Vol 49.

Knox, O. G. G., D. B. Nehl, T. Mor, G. N. Roberts, and V. Gupta. 2008. Genetically modified cotton has no effect on arbuscular mycorrhizal colonisation of roots. Field Crops Research 109:57-60.

Koide, R. T. and M. S. Peoples. 2012. On the nature of temporary yield loss in maize following canola. Plant and Soil 360:259-269.

Lendenmann, M., C. Thonar, R. L. Barnard, Y. Salmon, R. A. Werner, E. Frossard, and J. Jansa. 2011. Symbiont identity matters: Carbon and phosphorus fluxes between Medicago truncatula and different arbuscular mycorrhizal fungi. Mycorrhiza 
21:689-702.

Mack, K. M. L. and J. A. Rudgers. 2008. Balancing multiple mutualists: asymmetric interactions among plants, arbuscular mycorrhizal fungi, and fungal endophytes. Oikos 117:310-320.

McCain, K. N. S., G. W. T. Wilson, and J. M. Blair. 2011. Mycorrhizal suppression alters plant productivity and forb establishment in a grass-dominated prairie restoration. Plant Ecology 212:1675-1685.

McGonigle, T. P., M. H. Miller, D. G. Evans, G. L. Fairchild, and J. A. Swan. 1990. A new method which gives an objective-measure of colonization of roots by vesicular arbuscular mycorrhizal fungi. New Phytologist 115:495-501.

Middleton, E. L. and J. D. Bever. 2012. Inoculation with a native soil community advances succession in a grassland restoration. Restoration Ecology 20:218-226.

Munkvold, L., R. Kjoller, M. Vestberg, S. Rosendahl, and I. Jakobsen. 2004. High functional diversity within species of arbuscular mycorrhizal fungi. New Phytologist 164:357-364.

Natural Resources Conservation Service, USDA. 2012. Soil Survey Geographic (SSURGO) Database for Corvallis, Oregon. http://soildatamart.nrcs.usda.gov. Accessed 15 Nov. 2012.

NOAA ( National Oceanic and Atmospheric Administration). 2012. National Climatic Data Center. http://ncdc.noaa.gov/cdo-web. Accessed 15 Nov. 2012.

Phillips, J. M. and D. S. Hayman. 1970. Improved procedures for clearing roots and staining parasitic and vesicular-arbuscular mycorrhizal fungi for rapid assessment of infection. Transactions of the the British Mycological Society 55:158-160.

Rillig, M. C. 2004. Arbuscular mycorrhizae, glomalin, and soil aggregation. Canadian Journal of Soil Science 84:355-363.

Rosi-Marshall, E. J., J. L. Tank, T. V. Royer, M. R. Whiles, M. Evans-White, C. Chambers, N. A. Griffiths, J. Pokelsek, and M. L. Stephen. 2007. Toxins in transgenic crop byproducts may affect headwater stream ecosystems. Proceedings of the National Academy of Sciences of the United States of America 104:1620416208.

Sanchis, V. 2011. From microbial sprays to insect-resistant transgenic plants: History of the biospesticide Bacillus thuringiensis. A review. Agronomy for Sustainable Development 31:217-231.

Schaarschmidt, S., J. Kopka, J. Ludwig-Muller, and B. Hause. 2007. Regulation of arbuscular mycorrhization by apoplastic invertases: Enhanced invertase activity in the leaf apoplast affects the symbiotic interaction. Plant Journal 51:390-405.

Six, J., S. D. Frey, R. K. Thiet, and K. M. Batten. 2006. Bacterial and fungal contributions to carbon sequestration in agroecosystems. Soil Science Society of America Journal 70:555-569.

Smith, S. E. and D. J. Read. 2008. Mycorrhizal Symbiosis. 3rd edition. Academic Press, 
San Diego, CA.

Stinson, K. A., S. A. Campbell, J. R. Powell, B. E. Wolfe, R. M. Callaway, G. C. Thelen, S. G. Hallett, D. Prati, and J. N. Klironomos. 2006. Invasive plant suppresses the growth of native tree seedlings by disrupting belowground mutualisms. PLOS Biology 4:727-731.

Tan, F. X., J. W. Wang, Z. N. Chen, Y. J. Feng, G. L. Chi, and S. U. Rehman. 2011. Assessment of the arbuscular mycorrhizal fungal community in roots and rhizosphere soils of $B t$ corn and their non- $B t$ isolines. Soil Biology \& Biochemistry 43:2473-2479.

Tank, J. L., E. J. Rosi-Marshall, T. V. Royer, M. R. Whiles, N. A. Griffiths, T. C. Frauendorf, and D. J. Treering. 2010. Occurrence of maize detritus and a transgenic insecticidal protein (Cry1 Ab) within the stream network of an agricultural landscape. Proceedings of the National Academy of Sciences of the United States of America 107:17645-17650.

Tapp, H. and G. Stotzky. 1998. Persistence of the insecticidal toxin from Bacillus thuringiensis subsp. kurstaki in soil. Soil Biology \& Biochemistry 30:471-476.

Thonar, C., A. Schnepf, E. Frossard, T. Roose, and J. Jansa. 2011. Traits related to differences in function among three arbuscular mycorrhizal fungi. Plant and Soil 339:231-245.

Trappe, J. M., R. Molina, and M. Castellano. 1984. Reactions of mycorrhizal fungi and mycorrhiza formation to pesticides. Annual Review of Phytopathology 22:331359.

Turrini, A., C. Sbrana, M. P. Nuti, B. M. Pietrangeli, and M. Giovannetti. 2004. Development of a model system to assess the impact of genetically modified corn and aubergine plants on arbuscular mycorrhizal fungi. Plant and Soil 266:69-75.

USDA (United States Department of Agriculture). 2012. Adoption of Genetically Engineered Crops in the U.S.: Corn Varieties. http://www.ers.usda.gov/Data/BiotechCrops/ExtentofAdoptionTable1.htm. Accessed 15 Nov. 2012.

van der Heijden, M. G. A., T. Boller, A. Wiemken, and I. R. Sanders. 1998. Different arbuscular mycorrhizal fungal species are potential determinants of plant community structure. Ecology 79:2082-2091.

Verbruggen, E., E. E. Kuramae, R. Hillekens, M. de Hollander, E. T. Kiers, W. F. M. Roling, G. A. Kowalchuk, and M. G. A. van der Heijden. 2012. Testing potential effects of maize expressing the Bacillus thuringiensis Cry1 Ab endotoxin (Bt maize) on mycorrhizal fungal communities via DNA- and RNA-based pyrosequencing and molecular fingerprinting. Applied and Environmental Microbiology 78:7384-7392.

Veresoglou, S. D., B. D. Chen, and M. C. Rillig. 2012. Arbuscular mycorrhiza and soil nitrogen cycling. Soil Biology \& Biochemistry 46:53-62. 
Vogelsang, K. M. and J. D. Bever. 2009. Mycorrhizal densities decline in association with nonnative plants and contribute to plant invasion. Ecology 90:399-407.

Wang, B. and Y. L. Qiu. 2006. Phylogenetic distribution and evolution of mycorrhizas in land plants. Mycorrhiza 16:299-363.

Wehner, J., P. M. Antunes, J. R. Powell, T. Caruso, and M. C. Rillig. 2011. Indigenous arbuscular mycorrhizal fungal assemblages protect grassland host plants from pathogens. PLOS One 6.

Whiteside, M. D., K. K. Treseder, and P. R. Atsatt. 2009. The brighter side of soils: Quantum dots track organic nitrogen through fungi and plants. Ecology 90:100108.

Zwahlen, C., A. Hilbeck, P. Gugerli, and W. Nentwig. 2003. Degradation of the Cry1 Ab protein within transgenic Bacillus thuringiensis corn tissue in the field. Molecular Ecology 12:765-775. 


\section{Chapter 6. References}

Akiyama, K. and H. Hayashi. 2008. Plastid-derived strigolactones show the way to roots for symbionts and parasites. New Phytologist 178:695-698.

Akiyama, K., K. Matsuzaki, and H. Hayashi. 2005. Plant sesquiterpenes induce hyphal branching in arbuscular mycorrhizal fungi. Nature 435:824-827.

Brundrett, M. C., D. E. Enstone, and C. A. Peterson. 1988. A Berberine-Aniline Blue fluorescent staining procedure for suberin, lignin, and callose in plant-tissue. Protoplasma 146:133-142.

Castaldini, M., A. Turrini, C. Sbrana, A. Benedetti, M. Marchionni, S. Mocali, A. Fabiani, S. Landi, F. Santomassimo, B. Pietrangeli, M. P. Nuti, N. Miclaus, and M. Giovannetti. 2005. Impact of Bt corn on rhizospheric and soil eubacterial communities and on beneficial mycorrhizal symbiosis in experimental microcosms. Applied and Environmental Microbiology 71:6719-6729.

Cheeke, T. E., B. A. Pace, T. N. Rosenstiel, and M. B. Cruzan. 2011. The influence of fertilizer level and spore density on arbuscular mycorrhizal colonization of transgenic Bt 11 maize (Zea mays) in experimental microcosms. FEMS Microbiology Ecology 75:304-312.

Cheeke, T. E., T. N. Rosenstiel, and M. B. Cruzan. 2012. Evidence of reduced arbuscular mycorhizal fungal colonizaiton in multiple lines of $B t$ maize. American Journal of Botany 99:700-707.

De Micco, V. and G. Aronne. 2007. Combined histochemistry and autofluorescence for identifying lignin distribution in cell walls. Biotechnic \& Histochemistry 82:209216.

Deng, S. P. and M. A. Tabatabai. 1994. Colorimetric determination of reducing sugars in soils. Soil Biology \& Biochemistry 26:473-477.

Ferreira, L., J. C. Molina, C. Brasil, and G. Andrade. 2003. Evaluation of Bacillus thuringiensis bioinsecticidal protein effects on soil microorganisms. Plant and Soil 256:161-168.

Ferrol, N., J. M. Barea, and C. Azcon-Aguilar. 2002. Mechanisms of nutrient transport across interfaces in arbuscular mycorrhizas. Plant and Soil 244:231-237.

Flores, S., D. Saxena, and G. Stotzky. 2005. Transgenic Bt plants decompose less in soil than non-Bt plants. Soil Biology \& Biochemistry 37:1073-1082.

Garcia-Rodriguez, S., C. Azcon-Aguilar, and N. Ferrol. 2007. Transcriptional regulation of host enzymes involved in the cleavage of sucrose during arbuscular mycorrhizal symbiosis. Physiologia Plantarum 129:737-746.

Icoz, I. and G. Stotzky. 2008. Fate and effects of insect-resistant Bt crops in soil ecosystems. Soil Biology \& Biochemistry 40:559-586.

McGonigle, T. P., M. H. Miller, D. G. Evans, G. L. Fairchild, and J. A. Swan. 1990. A new method which gives an objective-measure of colonization of roots by vesicular arbuscular mycorrhizal fungi. New Phytologist 115:495-501. 
Nagahashi, G., D. D. Douds, and G. D. Abney. 1996. Phosphorus amendment inhibits hyphal branching of the VAM fungus Gigaspora margarita directly and indirectly through its effect on root exudation. Mycorrhiza 6:403-408.

Palm, C. J., D. L. Schaller, K. K. Donegan, and R. J. Seidler. 1996. Persistence in soil of transgenic plant produced Bacillus thuringiensis var kurstaki delta-endotoxin. Canadian Journal of Microbiology 42:1258-1262.

Pfeffer, P. E., D. D. Douds, G. Becard, and Y. Shachar-Hill. 1999. Carbon uptake and the metabolism and transport of lipids in an arbuscular mycorrhiza. Plant Physiology 120:587-598.

Saxena, D. and G. Stotzky. 2001. Bt corn has a higher lignin content than non-Bt corn. American Journal of Botany 88:1704-1706.

Sbrana, C. and M. Giovannetti. 2005. Chemotropism in the arbuscular mycorrhizal fungus Glomus mosseae. Mycorrhiza 15:539-545.

Schaarschmidt, S., M. C. Gonzalez, T. Roitsch, D. Strack, U. Sonnewald, and B. Hause. 2007a. Regulation of arbuscular mycorrhization by carbon. The symbiotic interaction cannot be improved by increased carbon availability accomplished by root-specifically enhanced invertase activity. Plant Physiology 143:1827-1840.

Schaarschmidt, S., J. Kopka, J. Ludwig-Muller, and B. Hause. 2007b. Regulation of arbuscular mycorrhization by apoplastic invertases: Enhanced invertase activity in the leaf apoplast affects the symbiotic interaction. Plant Journal 51:390-405.

Schaarschmidt, S., T. Roitsch, and B. Hause. 2006. Arbuscular mycorrhiza induces gene expression of the apoplastic invertase LIN6 in tomato (Lycopersicon esculentum) roots. Journal of Experimental Botany 57:4015-4023.

Shacharhill, Y., P. E. Pfeffer, D. Douds, S. F. Osman, L. W. Doner, and R. G. Ratcliffe. 1995. Partitioning of intermediary carbon metabolism in vesicular-arbuscular mycorrhizal leek. Plant Physiology 108:7-15.

Sharda, J. N. and R. T. Koide. 2008. Can hypodermal passage cell distribution limit root penetration by mycorrhizal fungi? New Phytologist 180:696-701.

Smith, S. E. and D. J. Read. 2008. Mycorrhizal Symbiosis. 3rd edition. Academic Press, San Diego, CA.

Solaiman, M. D. Z. and M. Saito. 1997. Use of sugars by intraradical hyphae of arbuscular mycorrhizal fungi revealed by radiorespirometry. New Phytologist 136:533-538.

Tapp, H. and G. Stotzky. 1995a. Dot-blot enzyme-linked-immunosorbent-assay for monitoring the fate of insecticidal toxins from Bacillus thuringiensis in soil Applied and Environmental Microbiology 61:602-609.

Tapp, H. and G. Stotzky. 1995b. Insecticidal activity of the toxins from Bacillus thuringiensis subspecies kurstaki and tenebronis adsorbed and bound on pure and soil clays Applied and Environmental Microbiology 61:1786-1790.

Tapp, H. and G. Stotzky. 1997. Monitoring the insecticidal toxins from Bacillus 
thuringiensis in soil with flow cytometry. Canadian Journal of Microbiology 43:1074-1078.

Tapp, H. and G. Stotzky. 1998. Persistence of the insecticidal toxin from Bacillus thuringiensis subsp. kurstaki in soil. Soil Biology \& Biochemistry 30:471-476.

Turrini, A., C. Sbrana, M. P. Nuti, B. M. Pietrangeli, and M. Giovannetti. 2004. Development of a model system to assess the impact of genetically modified corn and aubergine plants on arbuscular mycorrhizal fungi. Plant and Soil 266:69-75.

USDA (United States Department of Agriculture. 2012. Adoption of Genetically Engineered Crops in the U.S.: Corn Varieties. http://www.ers.usda.gov/Data/BiotechCrops/ExtentofAdoptionTable1.htm. Accessed 15 Nov. 2012.

Zadworny, M. and D. M. Eissenstat. 2011. Contrasting the morphology, anatomy and fungal colonization of new pioneer and fibrous roots. New Phytologist 190:213221.

Zeier, J., K. Ruel, U. Ryser, and L. Schreiber. 1999. Chemical analysis and immunolocalisation of lignin and suberin in endodermal and hypodermal/rhizodermal cell walls of developing maize (Zea mays L.) primary roots. Planta 209:1-12.

Zeier, J. and L. Schreiber. 1997. Chemical composition of hypodermal and endodermal cell walls and xylem vessels isolated from Clivia miniata - Identification of the biopolymers lignin and suberin. Plant Physiology 113:1223-1231.

Zeier, J. and L. Schreiber. 1998. Comparative investigation of primary and tertiary endodermal cell walls isolated from the roots of five monocotyledoneous species: chemical composition in relation to fine structure. Planta 206:349-361.

Zwahlen, C., A. Hilbeck, P. Gugerli, and W. Nentwig. 2003. Degradation of the Cry1Ab protein within transgenic Bacillus thuringiensis corn tissue in the field. Molecular Ecology 12:765-775. 


\section{Chapter 7. References}

Castaldini, M., A. Turrini, C. Sbrana, A. Benedetti, M. Marchionni, S. Mocali, A. Fabiani, S. Landi, F. Santomassimo, B. Pietrangeli, M. P. Nuti, N. Miclaus, and M. Giovannetti. 2005. Impact of $B t$ corn on rhizospheric and soil eubacterial communities and on beneficial mycorrhizal symbiosis in experimental microcosms. Applied and Environmental Microbiology 71:6719-6729.

Cheeke, T. E., M. B. Cruzan, and T. N. Rosenstiel. 2013. A field evaluation of arbuscular mycorrhizal fungal colonization in $B t$ and non-Bt maize. Applied and Environmental Microbiology 79:4078-4086.

Cheeke, T. E., B. A. Pace, T. N. Rosenstiel, and M. B. Cruzan. 2011. The influence of fertilizer level and spore density on arbuscular mycorrhizal colonization of transgenic Bt 11 maize (Zea mays) in experimental microcosms. FEMS Microbiology Ecology 75:304-312.

Cheeke, T. E., T. N. Rosenstiel, and M. B. Cruzan. 2012. Evidence of reduced arbuscular mycorhizal fungal colonizaiton in multiple lines of $B t$ maize. American Journal of Botany 99:700-707.

Pineyro-Nelson, A., J. Van Heerwaarden, H. R. Perales, J. A. Serratos-Hernandez, A. Rangel, M. B. Hufford, P. Gepts, A. Garay-Arroyo, R. Rivera-Bustamante, and E. R. Alvarez-Buylla. 2009. Transgenes in Mexican maize: molecular evidence and methodological considerations for GMO detection in landrace populations. Molecular Ecology 18:750-761.

Smith, S. E. and D. J. Read. 2008. Mycorrhizal Symbiosis. 3rd edition. Academic Press, San Diego, CA.

Turrini, A., C. Sbrana, M. P. Nuti, B. M. Pietrangeli, and M. Giovannetti. 2004. Development of a model system to assess the impact of genetically modified corn and aubergine plants on arbuscular mycorrhizal fungi. Plant and Soil 266:69-75. 